\title{
Two-Nucleon Short-Range Correlations in Light Nuclei
}

\author{
by \\ Reynier Cruz Torres \\ B.S., University of Florida (2015) \\ Submitted to the Department of Physics \\ in partial fulfillment of the requirements for the degree of \\ Doctor of Philosophy in Physics \\ at the \\ MASSACHUSETTS INSTITUTE OF TECHNOLOGY \\ June 2020 \\ (c) Massachusetts Institute of Technology 2020. All rights reserved.
}

Author

Department of Physics

April 3, 2020

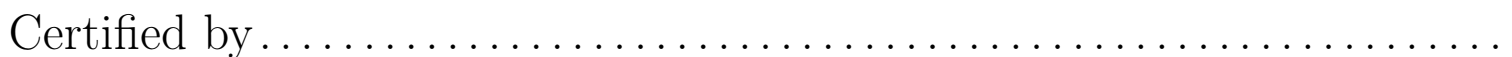

Or Hen

Assistant Professor

Thesis Supervisor

Accepted by

Nergis Mavalvala Associate Department Head, Physics 


\title{
Two-Nucleon Short-Range Correlations in Light Nuclei
}

\author{
by \\ Reynier Cruz Torres \\ Submitted to the Department of Physics \\ on April 3, 2020, in partial fulfillment of the \\ requirements for the degree of \\ Doctor of Philosophy in Physics
}

\begin{abstract}
Understanding the nucleon-nucleon $(N N)$ interaction is a fundamental task in nuclear physics, as $N N$-interaction models are a crucial input to modern nuclear structure calculations. While great progress has been made toward understanding this interaction, the available state-of-the-art models predict significantly different behaviors at short distances and high momenta (scale-and-scheme dependence), where two-nucleon Short-Range Correlations (SRCs) dominate the nuclear wave function. Thus, SRCs are a unique tool to constrain the $N N$ interaction and vice versa. SRCs are naturallyoccurring high-local-density $N N$ pairs that, as a result of their short-distance $(r \lesssim 1$ fm) repulsive interaction, fly apart with high momenta, hence populating momentum states above the Fermi level $\left(k \gtrsim k_{F} \approx 250 \mathrm{MeV} / c\right)$. The study of SRCs also has significant implications for other fields, such as the astrophysics of neutron stars and the behavior of cold atomic gasses. This thesis describes experimental and phenomenological studies of the short-distance / high-momentum structure of the $N N$ interaction through the study of SRCs and vice versa. Experimentally, I report the first measurement of the ${ }^{3} \mathrm{He}$ and ${ }^{3} \mathrm{H}\left(e, e^{\prime} p\right)$ reactions in Hall A of the Thomas Jefferson National Accelerator Facility in kinematics in which the measured cross sections should be sensitive to the underlying nucleon momentum distributions in the range 40 to $500 \mathrm{MeV} / c$. The resulting cross-section ratios and absolute cross sections were compared to momentum-distribution ratios and precise cross-section calculations respectively. Phenomenologically, I report the generalization of the Contact Formalism (GCF) to nuclear systems, which exploits scale separation and universality to describe nucleons at short distances and high momenta.
\end{abstract}

Thesis Supervisor: Or Hen

Title: Assistant Professor 


\section{Acknowledgments}

First of all, my eternal gratitude goes to my research supervisor, Prof. Or Hen. Or has provided me with the resources, network, and tools essential to get me to this point in my career. His unconcealable passion and interest for nuclear physics made me join him in the effort to find some order within the craziness that goes on inside the atomic nucleus. His guidance has been crucial in my formation as a physicist.

I am also grateful to Prof. Lawrence Weinstein and Douglas Higinbotham. I have had the privilege to interact directly with and get additional supervision from them. Beyond work, we have become great friends and their support has been enormously necessary to make my time in graduate school manageable. I'm also very thankful to Prof. Eliezer Piasetzky from whom I have also learned many things, especially that besides knowledge, creativity is essential to be a good physicist.

Or, Larry, and Eli have put together an outstanding group that I have always been proud to be a part of. I'm grateful to all the members of our group for their invaluable support. I want to especially acknowledge the tremendous guidance and help I have gotten from Florian Hauenstein and Dien Nguyen. I probably haven't interacted professionally with anyone else more than I have with Florian. From endless hours working in the hall to programming tips, Florian has played a big role in the formation of my career. While Dien joined our group recently, we have worked together very intensely to get to many of the results presented in this thesis. Thanks to all the graduate students from the group, especially Efrain Segarra and Afroditi Papadopoulou, who have become two of my best friends. Also thanks to Barak Schmookler who helped me a lot when I was getting started in various projects.

I needed a separate paragraph to express my gratitude to Prof. Axel Schmidt. During my time in graduate school, I have seen Axel successfully go from a graduate student, to a postdoc, to now an assistant professor. Axel's pedagogy, knowledge, and patience are extensive, and it has been a privilege to work with him so closely.

I'm grateful for all the input and guidance I have gotten from many physicists, especially Diego Lonardoni, Maria Piarulli, and Robert Wiringa for providing QMC 
calculations for the GCF analysis, and Prof. Nir Barnea and Ronen Weiss for their collaboration in our joint work to extract nuclear contacts and test the universality of the GCF. Thanks also to Prof. Jacek Golak and Prof. Misak Sargsian for providing us with their $A=3$ calculations. I am also thankful to Prof. Gerald Miller for his tremendous help in the extraction of nuclear correlation functions, and Prof. Werner Boeglin for his guidance in implementing the $A=3$ cross section in the simulation package used in our analysis.

I'm grateful to all the people I have met during my time in graduate school. Thanks to my MIT classmates, who worked with me through courses and qualifying exams, especially my dear friends: Sameer Abraham, Thomas Boettcher, Jasmine Brewer, Charles Epstein, Dahlia Klein, Cyprian Lewandowski, Elizabeth Tolman, Constantin Weisser, Yunjie Yang, and Evan Zayas. Thanks to Cathy Modica for making MIT a home. Thanks to all the members of the tritium collaboration, especially the tritium students and postdocs: Sheren Alsalmi, Jason Bane, Scott Barcus, Jonathan Castellanos, Tyler Hague, Tyler Kutz, Shujie Li, Hanjie Liu, Evan McClellan, Michael Nycz, Bishnu Pandey, Nathaly Santiesteban, and Tong Su. Furthermore, I want to express my gratitude to Dave Meekins, who got the tritium target up and running. Lastly, thanks to everyone who took time to read my thesis and suggest valuable comments, especially to my friend Carlos Ayerbe Gayoso.

Thanks to the people that pushed me since high school to the doors of graduate school. I'm especially thankful to my undergraduate advisor, Prof. Heather Ray, and to Prof. Eric Prebys for giving me my very first research opportunities in the United States.

I'm very grateful to my entire family for their continuous support, encouragement, visits, and advise. I'm especially thankful to my mother Yakeline Torres Garcia (my biggest fan and supporter) and my step-father Michel Bormey Garcia for being by my side every step of the way. I'm very thankful to my father Juan R. Cruz Alonso for always encouraging me to pursue my professional goals. I'm also eternally thankful to my grandmother Daisy Garcia Nuñez, who would not let my coffee supply get low, and to my uncle Mario Torres Garcia, who loves to engage in physics conversations 
with me. Thanks as well to my grandparents Carola Alonso Echazábal, Osvaldo Cruz Alfonso, and Ramón Torres Alvarez. I'm also thankful to my lifelong friends: Alejandro Carrasco Gonzalez, Robin Feito Linares, Javier León Mendez, and Claudia Llerena Fernández, whom I met at a very young age and have always, to this day, had my back and set a great example for me to follow.

Last, but definitely not least, my profound gratitude to my dearest friend, my wife, and the love of my life, Ross M. Zamora Rios. Her adamant hard work and dedication as a student have set a very high bar for me to follow. Her love, care, and support have made my time in graduate school very joyful. Her constant encouragement and the extraordinary example that she has set have given me a lot of motivation and strength to never give up. 


\section{Contents}

1 Introduction 21

1.1 The $N N$ interaction . . . . . . . . . . . . . . . 22

1.2 Quantum Monte Carlo (QMC) . . . . . . . . . . . 25

1.3 Two-Nucleon Short-Range Correlations (SRCs) . . . . . . . . . . 27

1.4 Scope of this work . . . . . . . . . . . . . . 35

1.4.1 A word on notation changes ........... 36

2 Quasi-Elastic Electron Scattering off Light-Nuclei 37

2.1 Introduction . . . . . . . . . . . . . . . . 37

2.2 Quasi-elastic $\left(e, e^{\prime} p\right)$ scattering ................... 38

2.2.1 Quasi-elastic $\left(e, e^{\prime} p\right)$ scattering off $A=3$ nuclei ..... 41

2.2.2 Suppression of non-QE reaction mechanisms . . . . . . 44

2.2.3 $\left(e, e^{\prime} p\right)$ cross-section calculations for few-body systems . . . 46

2.2.4 Previous $A=3$ electron-scattering measurements . . . . . . 47

3 Measurement of $\left(e, e^{\prime} p\right)$ Scattering off ${ }^{3} \mathbf{H}$ And ${ }^{3} \mathbf{H e}$

[Phys. Lett. B 797, 134890 (2019), arXiv: 2001.07230 (2020)] 51

3.1 Experimental setup . . . . . . . . . . . . . . 52

3.1 .1 JLab accelerator .................... 52

3.1.2 Hall-A beam line . . . . . . . . . . . . . . 53

3.1.3 Tritium target assembly . . . . . . . . . . 56

3.1.4 High-Resolution Spectrometers (HRS) . . . . . . . . 58

3.1.5 Trigger setup and efficiency ............. 64 
3.2 Simulation studies $\ldots \ldots \ldots \ldots \ldots \ldots$

3.3 Calibrations . . . . . . . . . . . . . . . . . . . . 68

3.3 .1 BCM calibration . . . . . . . . . . . . 69 69

3.3.2 HRS optics calibration . . . . . . . . . . . . . . 71

3.4 Luminosity check . . . . . . . . . . . . . . . . . 77

3.5 Data analysis . . . . . . . . . . . . . . . . 79

3.5 .1 Event selection $\ldots \ldots \ldots \ldots \ldots \ldots$. . . . . . . . . . .

3.5.2 Data-SIMC comparison . . . . . . . . . . . . . 83

$3.6{ }^{3} \mathrm{He} /{ }^{3} \mathrm{H}\left(e, e^{\prime} p\right)$ cross-section ratio $\ldots \ldots \ldots \ldots \ldots \ldots$

3.6.1 Density ('boiling') corrections . . . . . . . . . . . 85

$3.6 .2{ }^{3} \mathrm{H}$-decay correction . . . . . . . . . . . . . . . 86

3.6.3 Radiative corrections . . . . . . . . . . . . . . . . 87

3.6.4 Bin-migration corrections . . . . . . . . . . . 90

3.6.5 $E_{m i s s}$-acceptance corrections . . . . . . . . . . 91

3.6 .6 Final corrections . . . . . . . . . . . . . . 92

3.6 .7 Systematic uncertainties . . . . . . . . . . . . . 9 93

3.6 .8 Final results . . . . . . . . . . . . . . . . . . . . 94

$3.7{ }^{3} \mathrm{He},{ }^{3} \mathrm{H}\left(e, e^{\prime} p\right)$ absolute cross sections $\ldots \ldots \ldots \ldots \ldots$

3.7.1 Phase-space and acceptance correction factors . . . . . . 100

3.7 .2 Radiative corrections . . . . . . . . . . . . . . . . . . 101

3.7.3 Bin-migration corrections $\ldots \ldots \ldots \ldots \ldots$

3.7.4 Bin-centering corrections . . . . . . . . . . . . . . . 104

$3.7 .5{ }^{3} \mathrm{H}$-decay correction . . . . . . . . . . . . . . 106

3.7 .6 Final corrections . . . . . . . . . . . . . 106

3.7.7 Systematic uncertainties . . . . . . . . . . . . . . 107

3.7.8 AV18 to CD-Bonn effective conversion . . . . . . . . . 108

3.7 .9 Final results . . . . . . . . . . . . . . . . . . 109

\section{Generalized Contact Formalism}

[Phys. Lett. B 780, 211 (2018), arXiv:1907.03658 (2019), Phys. Lett. B 785, 
$304(2018)]$

4.1 Introduction . . . . . . . . . . . . . . . . . 115

4.2 Contact Formalism in atomic systems . . . . . . . . . . . . . 116

4.3 Generalization to nuclear systems . . . . . . . . . . . . . . 117

4.3.1 Coordinate densities and momentum distributions . . . . . . 118

4.3 .2 Normalization conventions . . . . . . . . . . . . . . . 122

4.4 Benchmarking the GCF . . . . . . . . . . . . . . . . . . . . . 122

4.4.1 Contacts from 2-body coordinate densities . . . . . . . . 123

4.4.2 Contacts from 2-body momentum distributions . . . . . . . 124

4.4.3 Contact extraction from experimental data . . . . . . . . 132

4.4.4 AV18 results and discussion . . . . . . . . . . . . . . 132

4.4.5 Contact verification with 1-body momentum distributions . . 133

4.4.6 SRCs and the $S T=11$ channel $\ldots \ldots \ldots \ldots$

4.5 Testing scale-and-scheme independence with the GCF . . . . . . . 135

4.5.1 Scale and scheme independence of nuclear contacts and positionmomentum equivalence of SRCs . . . . . . . . . . . . . 139

4.5.2 Absolute contacts . . . . . . . . . . . . . . . . . . . 142

4.5.3 Systematic uncertainties . . . . . . . . . . . . . 146

4.6 Applications of the GCF . . . . . . . . . . . . . . . . . 147

4.7 Nuclear correlation functions . . . . . . . . . . . . . . . . . 148

4.7.1 Correlation function definition . . . . . . . . . . . . . . 149

4.7.2 Fully-correlated 2-body coordinate density . . . . . . . . 149

4.7.3 Uncorrelated 2-body coordinate density and the Pauli exclusion principle . . . . . . . . . . . . . . . 152

4.7.4 Correlation function results and discussion . . . . . . 154

5 Summary and Conclusions 157

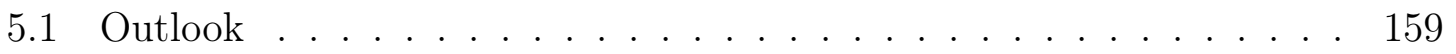

A HRS optics study 161

A.1 High-order HRS optics checks . . . . . . . . . . . . . . . 161 
A.2 $0^{t h}$-order HRS optics check . . . . . . . . . . . . . 165

B ${ }^{3} \mathbf{H e}$ and ${ }^{3} \mathbf{H}\left(e, e^{\prime} p\right)$ kinematical distributions 169

C Additional Chapter 3 Tables

D Generalized Contact Formalism 1-body momentum distributions 185

E Generalized Contact Formalism ${ }^{4}$ He Fit Examples 189 


\section{List of Figures}

1-1 One-pion exchange contribution to the $N N$ interaction. . . . . . . 22

1-2 Central and tensor channels from four different $N N$ potentials as a function of nucleon-nucleon separation. . . . . . . . . . 23

1-3 Spectroscopic strengths for valence orbitals from electron-induced protonknockout compared to Mean-Field theory expectations. . . . . . . 28

1-4 Inclusive $\left(e, e^{\prime}\right)$ per-nucleon cross-section ratios as a function of $x_{B}$. $\quad 30$

1-5 ${ }^{3} \mathrm{He}\left(e, e^{\prime} p\right)$ reduced cross section as a function of missing momentum. 30

1-6 Center-of-mass momentum distribution of SRC pairs. . . . . . . 32

1-7 $n p$ dominance of SRCs. . . . . . . . . . . . . . . . 32

1-8 Effect of pair center-of-mass momentum on relative $p p$-to- $p n$ abundance. 34

1-9 Fraction of high-momentum nucleons in nucleus $A$ relative to carbon as a function of neutron excess. . . . . . . . . . . . . 34

2-1 Electron-nucleus scattering cross section vs. transfer energy. . . . . . 38

2-2 Diagram for $\left(e, e^{\prime} p\right)$ reaction kinematics. . . . . . . . . . . . 39

2-3 Reaction mechanisms of interest. . . . . . . . . . . . . 41

2-4 Ratio of ${ }^{3} \mathrm{He}$ neutron to ${ }^{3} \mathrm{H}$ proton momentum distributions as a function of nucleon momentum calculated using three different $N N$ models. 42

2-5 $A=3$ AV18 spectral function by C. Ciofi degli Atti and L. P. Kaptari. 44

2-6 Non-QE reaction mechanisms with the same experimental signature as the diagrams in Fig. 2-3. . . . . . . . . . . . . . . 45

2-7 Ratio of calculated ${ }^{3} \mathrm{He}\left(e, e^{\prime} p\right)$ full cross section to the PWIA cross section as a function of $\theta_{r q} \ldots \ldots \ldots \ldots$ 
2-8 ${ }^{3} \mathrm{He}\left(e, e^{\prime} p\right)$ pn cross-section as a function of missing energy. . . . . . 48

3-1 Aerial view of the Continuous Electron Beam Accelerator Facility (CEBAF) at JLab. . . . . . . . . . . . . . . 52

3-2 Absolute beam-energy measurement using the arc method. . . . . . . 555

3-3 Schematic of the Hall-A Beam Current Monitor (BCM). . . . . . . . 56

3-4 Target Assembly. . . . . . . . . . . . . . . . 57

3-5 Schematic of the gas target cell. . . . . . . . . . . . 57

3-6 Hall-A experimental setup. . . . . . . . . . . . . . . . 59

3-7 Schematic of the HRS detector package. . . . . . . . . . 60

3-8 Schematic layout for the two Vertical Drift Chambers (VDC) in each Hall-A HRS. . . . . . . . . . . . . . . . . . 61 61

3-9 Schematic layout for the hodoscopes in each Hall-A HRS. . . . . . . . 62

3-10 Venn diagram illustrating the relationship between different triggers. . 65

3-11 Unser and RF-cavity calibration procedures. . . . . . . . . . . . . . 70

3-12 Geometric and reconstructed Sieve-slit collimator pattern. . . . . . . 73

3-13 $E_{\text {miss }}$ dependence as a function of LHRS $\theta_{t g}$ for $\mathrm{H}\left(e, e^{\prime} p\right)$ elastic scattering. 74

3-14 Difference between proton scattering angle measured by RHRS (reco), and calculated from different pairs of variables (calc) . . . . . . 76

3-15 Missing energy in $\mathrm{d}\left(e, e^{\prime} p\right)$ QE scattering before and after optics corrections. ........................ 77

3-16 Luminosity check. . . . . . . . . . . . . . . . 79

3-17 HRS acceptance from a SIMC simulation and effect of acceptance cuts. 80

3-18 z-component of the interaction vertex reconstructed with the LHRS vs RHRS before and after vertex cuts in the low- $p_{m i s s}$ kinematical setting. 81

3-19 Particle-identification criteria for electrons and protons. . . . . . 82

3-20 Comparison between ${ }^{3} \mathrm{H}\left(e, e^{\prime} p\right)$ measured and simulated distributions for the low- $p_{\text {miss }}$ kinematical setting. . . . . . . . . . . . . 83

3-21 Comparison between ${ }^{3} \mathrm{He} E_{\text {miss }}$ distributions from measurement and calculations with and without $\mathrm{FSI}_{23}$............ 84 
3-22 Missing-momentum dependence of the measured $\left(e, e^{\prime} p\right){ }^{3} \mathrm{He} /{ }^{3} \mathrm{H}$ normalized event-yield ratios. ............... 87

3-23 Leading-order diagram in the QE electron-proton interaction. . . . . 88

3-24 Some examples of higher-order electron-proton scattering diagrams. . 88

3-25 Simulated missing-energy spectrum for ${ }^{3} \mathrm{He}$ in the low- $p_{\text {miss }}$ setting with and without radiative effects. . . . . . . . . . . . 89

3-26 Toy model to illustrate the spectrometer limited $E_{\text {miss }}$ acceptance. . . 91

3-27 Corrections applied in the ratio analysis. . . . . . . . . 93

$3-28{ }^{3} \mathrm{He} /{ }^{3} \mathrm{H}$ cross-section ratio as a function of $p_{\text {miss }}$ compared with different sets of momentum-distribution ratios. . . . . . . . . 95

3-29 Effect of single-rescattering FSI in the ${ }^{3} \mathrm{He} /{ }^{3} \mathrm{H}$ ratio as a function of missing momentum for the high- $p_{m i s s}$ kinematical setting. . . . . . . 97

3-30 $E_{\text {miss }}$ integration limits for each $p_{\text {miss }}$ bin. . . . . . . . . . . . . . 100

3-31 Relative 2- and 3-body breakup contributions to the measured yield as a function of $E_{\text {miss. }} \ldots \ldots \ldots \ldots$

3-32 Radiative correction factors integrated up to different values of $E_{\text {miss }}$. 103

3-33 Bin-centering correction factors for the low- $p_{\text {miss }}$ kinematical setting. 105

3-34 Same as Fig. 3-33 for the high- $p_{\text {miss }}$ kinematical setting. . . . . . . . 105

3-35 Size of corrections applied in the absolute analysis. . . . . . . . . . 107

3-36 Ratio of the proton momentum distributions obtained using the CDBonn + TM and AV18+UIX potentials for ${ }^{3} \mathrm{He}$ and ${ }^{3} \mathrm{H}$. . . . . . . . . 109

3-37 Absolute cross section as a function of $p_{\text {miss }}$ for ${ }^{3} \mathrm{He}$ and ${ }^{3} \mathrm{H}$. . . . . . 110

3-38 Ratio of the experimental cross section to different PWIA calculations

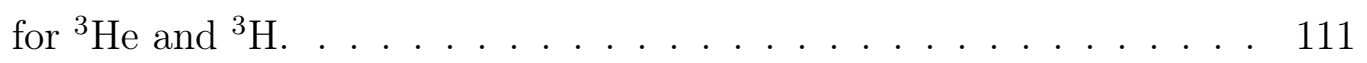

3-39 Ratio of the experimental cross section to the Sargsian-FSI calculations for ${ }^{3} \mathrm{He}$ and ${ }^{3} \mathrm{H}$ in the high- $p_{\text {miss }}$ kinematical setting. . . . . . . . . . 112

3-40 Ratio of the total $A=3$ experimental cross section $\left({ }^{3} \mathrm{He}+{ }^{3} \mathrm{H}\right)$ to different calculations as a function of missing momentum. The dark- and light-gray bands correspond to the 10 and $20 \%$ agreement intervals respectively. . . . . . . . . . . . . . . . . . 113 
4-1 NN asymptotic densities calculated using the AV18 NN potential. . 118

4-2 QMC Oxygen-16 2-body coordinate densities $\rho_{N N}^{16} \mathrm{O}\left(R_{0}, r\right) \ldots \ldots$

$4-3$ Oxygen-16 contacts $C_{N N}^{\alpha}(R) \ldots \ldots \ldots \ldots \ldots$. . . . . . . . . . . . . . .

4-4 Short-distance universality of AV18 2-body coordinate densities. . . . 123

4-5 High-momentum universality of AV18 2-body momentum distributions. 126

4-6 Toy model 2-body momentum distribution as a function of center-ofmass $(Q)$ and relative $(q)$ momenta. . . . . . . . . . . . 130

4-7 ${ }^{4} \mathrm{He}$ SRC $p p / p n$ fraction as a function of $q \ldots \ldots \ldots$. . . . . . . . . 131

4-8 Comparison between 1-body momentum distributions from VMC and from the GCF. . . . . . . . . . . . . . . . . . . . . . . . . . 134

4-9 2-body coordinate densities and momentum distributions calculated using different $N N$ potential models. . . . . . . . . . . . 136

4-10 Universal asymptotic densities for spin-1 pn and spin-0 pp pairs in coordinate- and momentum-space for five different $N N$ potentials. . . 137

4-11 Short-distance and high-momentum universality of 2-body coordinate densities and momentum distributions for different $N N+3 N$ interactions.138

4-12 Ratios of $p n$ spin-1 nuclear contacts to deuterium, ${ }^{4} \mathrm{He}$, and of $p p$ spin-0 nuclear contacts to ${ }^{4} \mathrm{He}$ for different nuclei. . . . . . . . . . . . . . . . . 140

4-13 Ratios of ${ }^{4} \mathrm{He} p p$-to- $p n$ back-to-back $(Q=0)$ pairs from experiment and theory as a function of relative momentum. . . . . . . . . . . . 142

4-14 Absolute values of the $p n$ spin-1 and $p p$ spin-0 contacts extracted in coordinate and momentum space from QMC calculations. . . . . . . . 144

4-15 Ratios of coordinate- to momentum-space nuclear contacts for pn spin1 and $p p$ spin-0 pairs for different nuclei. . . . . . . . . . . . . 144

4-16 Ratios of contacts extracted from VMC 2-body coordinate densities to contacts extracted from DMC and EXT 2-body coordinate densities. 147

4-17 Comparison between the 2-body coordinate density from the model from equation 4.34 and that from CVMC for ${ }^{16} \mathrm{O}$ and ${ }^{40} \mathrm{Ca} . . . . .151$ 4-18 Effect of including or neglecting the Pauli-exclusion term in equation 4.39, 154 
4-19 Results from the isospin-decomposed ${ }^{16} \mathrm{O}$ and ${ }^{40} \mathrm{Ca}$ correlation functions and comparison to several other calculations. . . . . . . . . 155

A-1 Elastic $\mathrm{H}\left(e, e^{\prime} p\right)$ invariant mass as a function of LHRS optics variables. 161 A-2 Same as Fig. A-1 as a function of RHRS optics variables. . . . . . . 162

A-3 Elastic $\mathrm{H}\left(e, e^{\prime} p\right)$ missing energy as a function of LHRS optics variables. 162

A-4 Same as Fig. A-3 as a function of RHRS optics variables. . . . . . 162

A-5 Elastic $\mathrm{H}\left(e, e^{\prime} p\right) x$ component of missing momentum as a function of LHRS optics variables. . . . . . . . . . . . . . . . . 163

A-6 Same as Fig. A-5 as a function of RHRS optics variables. . . . . . 163 163

A-7 Elastic $\mathrm{H}\left(e, e^{\prime} p\right)$ y component of missing momentum as a function of LHRS optics variables. . . . . . . . . . . . . . . . . 163

A-8 Same as Fig. A-7 as a function of RHRS optics variables. . . . . . 164

A-9 Elastic $\mathrm{H}\left(e, e^{\prime} p\right) z$ component of missing momentum as a function of LHRS optics variables. . . . . . . . . . . . . . . . . . 164

A-10 Same as Fig. A-9 as a function of RHRS optics variables. . . . . . 165

A-11 Counts vs. scattered electron momentum. . . . . . . . . . 165

A-12 Counts vs. electron scattering angle. . . . . . . . . . . . 166

A-13 Counts vs. knocked-out proton momentum. . . . . . . . . 166

A-14 Counts vs. invariant mass. . . . . . . . . . . . . . 166

A-15 Counts vs. missing momentum $x$ component. . . . . . . . . 167

A-16 Counts vs. missing momentum $y$ component. . . . . . . . . . 167

A-17 Counts vs. missing momentum $z$ component. . . . . . . . . . 167

B-1 Number of counts vs. $p_{\text {miss }}$ for the low- and high- $p_{\text {miss }}$ kinematical settings. . . . . . . . . . . . . . . . . 170

B-2 Same as Fig. B-1, as a function of $E_{\text {miss }} \ldots \ldots \ldots \ldots \ldots$

B-3 Same as Fig. B-2, for ${ }^{3} \mathrm{He}$ only, with separation of the SIMC yield to contributions to $2 \mathrm{bbu}$ and 3bbu channels. . . . . . . . . . . . 170

B-4 Same as Fig. B-1, as a function of $\theta_{r q} \ldots \ldots \ldots \ldots \ldots \ldots$

B-5 Same as Fig. B-1, as a function of $Q^{2} \ldots \ldots \ldots \ldots \ldots$ 
B-6 $p_{\text {miss }}$ dependence of the measured over simulated ${ }^{3} \mathrm{He} /{ }^{3} \mathrm{H}$ yield ratios from Fig. B-1 . . . . . . . . . . . . . . . . . . . . . . 171

B-7 ${ }^{3}$ He $E_{\text {miss }}$ vs. $p_{\text {miss }}$ measured and simulated distributions. . . . . . . 172

B-8 Same as Fig. B-7 only for ${ }^{3} \mathrm{H}$. . . . . . . . . . . . . 172

B-9 ${ }^{3}$ He $Q^{2}$ vs. $p_{\text {miss }}$ measured and simulated distributions. . . . . . . . 173

B-10 Same as Fig. B-9 only for ${ }^{3}$ H. . . . . . . . . . . . . . 173

B-11 ${ }^{3} \mathrm{He} \theta_{r q}$ vs. $p_{\text {miss }}$ measured and simulated distributions. . . . . . . . 174

B-12 Same as Fig. B-11 only for ${ }^{3}$ H. . . . . . . . . . . . . . 174

B-13 ${ }^{3}$ He $p_{\text {miss }}$ vs. $x_{B}$ measured and simulated distributions. . . . . . . . . 175

B-14 Same as Fig. B-14 only for ${ }^{3}$ H. . . . . . . . . . . . . . 175

D-1 Comparison between the ${ }^{4} \mathrm{He}$ 1-body momentum distributions from VMC and GCF. . . . . . . . . . . . . . . . 186

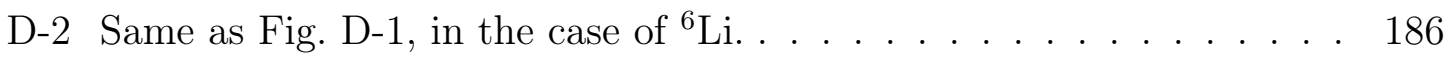

D-3 Same as Fig. D-1, in the case of ${ }^{8}$ Be. . . . . . . . . . . . . 187

D-4 Same as Fig. D-1, in the case of ${ }^{10}$ B. . . . . . . . . . . . . . . 187

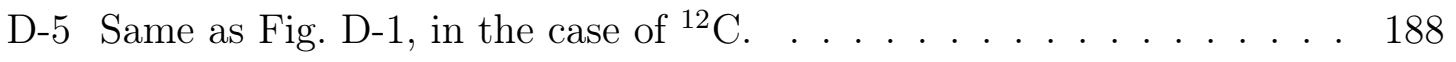

D-6 Comparison between the ${ }^{16} \mathrm{O}$ and ${ }^{40} \mathrm{Ca}$ 1-body momentum distributions from VMC and GCF with contacts extracted in coordinate- space. . . 188

E-1 ${ }^{4}$ He fits in coordinate- and momentum-space for the AV4'+UIX ${ }_{c}$ potential. . . . . . . . . . . . . . . . . . . . 189

E-2 Same as Fig. E-1 for the $\mathrm{N}^{2} \mathrm{LO}(1.0 \mathrm{fm}, E \mathbb{1})$ potential. . . . . . . . . 190

E-3 Same as Fig. E-1 for the $\mathrm{N}^{2} \mathrm{LO}(1.0 \mathrm{fm}, E \tau)$ potential. . . . . . . . . . 190

E-4 Same as Fig. E-1 for the $\mathrm{N}^{2} \mathrm{LO}(1.2 \mathrm{fm}, E \mathbb{1})$ potential. . . . . . . . . . 190

E-5 Same as Fig. E-1 for the $\mathrm{N}^{2} \mathrm{LO}(1.2 \mathrm{fm}, E \tau)$ potential. . . . . . . . . . 191 


\section{List of Tables}

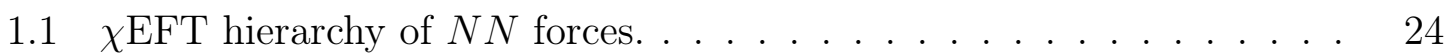

2.1 List of available ${ }^{3} \mathrm{He}$ and ${ }^{3} \mathrm{H}\left(e, e^{\prime} p\right)$ cross-section calculations. . . . 47

3.1 Properties of the gas targets used in the experiment. . . . . . . 58

3.2 Hall-A HRS nominal momentum and angular acceptance. . . . . . . 59

3.3 Trigger setup. . . . . . . . . . . . . . . . . . . . . 65

3.4 Kinematical settings used in the experiment. . . . . . . . . 6 68

3.5 RF-cavity calibration parameters. . . . . . . . . . . . . 71

3.6 Density correction factors at $I=22.5 \mu \mathrm{A} \ldots \ldots \ldots \ldots$

3.7 Sampling limits for systematic sensitivity study. . . . . . . . . . 94

3.8 Overall systematic uncertainties in the extraction of the ${ }^{3} \mathrm{He} /{ }^{3} \mathrm{H}\left(e, e^{\prime} p\right)$ crosssection ratio. . . . . . . . . . . . . . . . . 94

$3.9 V_{B}$ (acceptance-corrected detected phase-space) correction values. . . 101

3.10 Two- and three-body breakup coefficients for the low- and high- $p_{m i s s}$ kinematical settings. . . . . . . . . . . . . . . . . 102

3.11 Overall systematic uncertainties in the extraction of the ${ }^{3} \mathrm{He}\left(e, e^{\prime} p\right)$ and ${ }^{3} \mathrm{H}\left(e, e^{\prime} p\right)$ absolute cross sections. . . . . . . . . . . . . 108

$3.12 N N$ potentials used in the different cross-section calculations. . . . 109

4.1 AV18 nuclear contacts for a variety of nuclei in coordinate $(r)$ and momentum $(k)$ space. . . . . . . . . . . . . . . . . 125

4.2 Nuclear-contact values for different $N N+3 N$ models in coordinate $(r)$ and momentum $(k)$ space. . . . . . . . . . . . . . . 145 
4.3 Parameters describing $\mathbb{F}(r) \ldots \ldots \ldots \ldots \ldots \ldots$

C.1 Acceptance-integrated cross section values and corresponding kinematical points for the low- $p_{\text {miss }}$ kinematical setting. . . . . . . . . . 178

C.2 Same as Table C.1 for the high- $p_{\text {miss }}$ kinematical setting. . . . . . . 179

C.3 Point cross section values and corresponding kinematical points for the low- $p_{\text {miss }}$ kinematical setting. . . . . . . . . . . . . . . 180

C.4 Same as Table C.3 for the high- $p_{\text {miss }}$ kinematical setting. . . . . . . 181

C.5 Measured ratios of ${ }^{3} \mathrm{He} /{ }^{3} \mathrm{H}\left(e, e^{\prime} p\right)$ normalized event yields and cross sections with uncertainties. . . . . . . . . . . . . . 182

C.6 Values of the $E_{m i s s}$-integrated ${ }^{3} \mathrm{He}$ experimental cross section, corrections, and uncertainties. . . . . . . . . . . . . 183

C.7 Same as Table C.6 for ${ }^{3} \mathrm{H} . \ldots \ldots \ldots$ 


\section{Chapter 1}

\section{Introduction}

The scientific quest to understand how two free nucleons interact with each other and how this interaction conspires to form the complex atomic nucleus has been at the center of nuclear physics since the birth of this field in 1932 after the discovery of the neutron [1]. In 1953 Hans A. Bethe famously said that, to this purpose, "...physicists have devoted $[\ldots]$ probably more man-hours than have been given to any other scientific question in the history of mankind". Due to the complex nature of the Nucleon-Nucleon $(N N)$ interaction, this statement is as valid today as it was back then, and the quest is far from over. The complexity of the task stems from the fact that the $N N$ interaction is not fundamental, since it is the residual strong interaction that binds quarks together to form nucleons, and can be seen reflected in that there is no unified way of describing it! The $N N$ interaction is a crucial input to modern studies of nuclear structure and reactions, as well as of the properties of dense astrophysical objects such as neutron stars.

This work studies the $N N$ interaction up to high momenta and short distances, where Two-Nucleon Short-Range Correlations (SRCs) dominate the nuclear wave function. This chapter reviews our current knowledge of the $N N$ interaction and SRCs. Chapter 2 presents the theoretical background to understand electron-induced quasi-elastic (QE) proton-knockout $\left(e, e^{\prime} p\right)$ measurements in light nuclei, with empha-

\footnotetext{
${ }^{1}$ For example, there are over $40 N N$ models. Furthermore, first-principle techniques like Effective Field Theory and Lattice QCD make predictions for only limited kinematic ranges.
} 
sis on identifying kinematics that are sensitive to the nuclear ground state. Chapter 3 describes an experiment carried out in such kinematics at the Thomas Jefferson National Accelerator Facility (JLab) with the goal to constrain precise models of the three-nucleon-system cross section up to high initial nucleon momenta. Chapter 4 presents an effective model to describe the nuclear wave function at short distances and high momenta, where traditional nuclear structure models fail. Applications of this model are also presented. Finally, chapter 5 summarizes the work presented in the thesis, and then presents the conclusions.

\section{$1.1 \quad$ The $N N$ interaction}

The first attempt at describing the $N N$ interaction is attributed to Yukawa, who proposed that the interaction is mediated by massive-meson exchanges [2]. Fig. 1-1] shows a proton-neutron scattering via one-pion exchange (OPE).

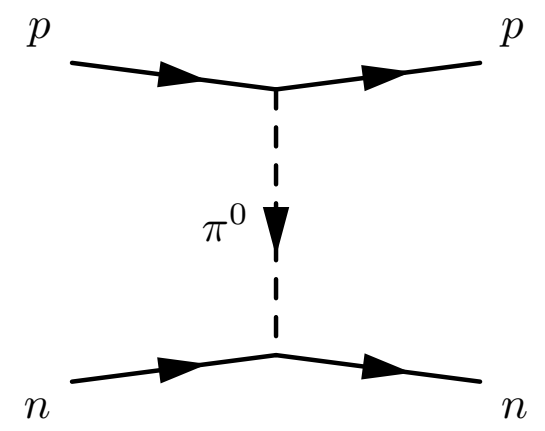

Figure 1-1: One-pion exchange contribution to the $N N$ interaction.

Modern (post-1990) $N N$ models still make use of the OPE channel to describe the long-range $(r \gtrsim 2 \mathrm{fm})$, as well as the tensor parts of the interaction. Two of the most used categories of $N N$ potentials are: 1) phenomenological, and 2) Chiral Effective Field Theory $(\chi \mathrm{EFT})$ (for a detailed review see, e.g., [3]).

\section{Phenomenological potentials}

Phenomenological potentials describe the medium- $(1 \lesssim r \lesssim 2 \mathrm{fm})$ and short- $(r \lesssim$ $1 \mathrm{fm})$ ranges of the $N N$ interaction via phenomenological parametrizations $(\approx 45$ 
parameters) that are fitted to $N N$ elastic-scattering data in the form of phase shifts and deuteron binding energy. These fits are carried out up to relative momenta that correspond to the pion-production threshold $\left(E_{l a b} \approx 350 \mathrm{MeV}\right)$, with no significant constraints at higher energies. The selected parametrization varies from model to model. The success of these potentials can be seen by its reproduction of the data it is fitted to, with $\chi_{\text {red }}^{2} \approx 1$. Fig. 1-2 shows the central and tensor channels of two phenomenological potentials: the Argonne $v$-18 (AV18) potential (which is described by 18 operators) [4, and its reduction AV4' (which is a reprojection of AV18's first four channels: central, spin, isospin, and spin-isospin) [5]. The tensor force is intentionally left out of the AV4' model. These two potentials are "hard interactions", with a strong short-distance repulsive core and a significant probability for nucleons to reach highmomentum states.

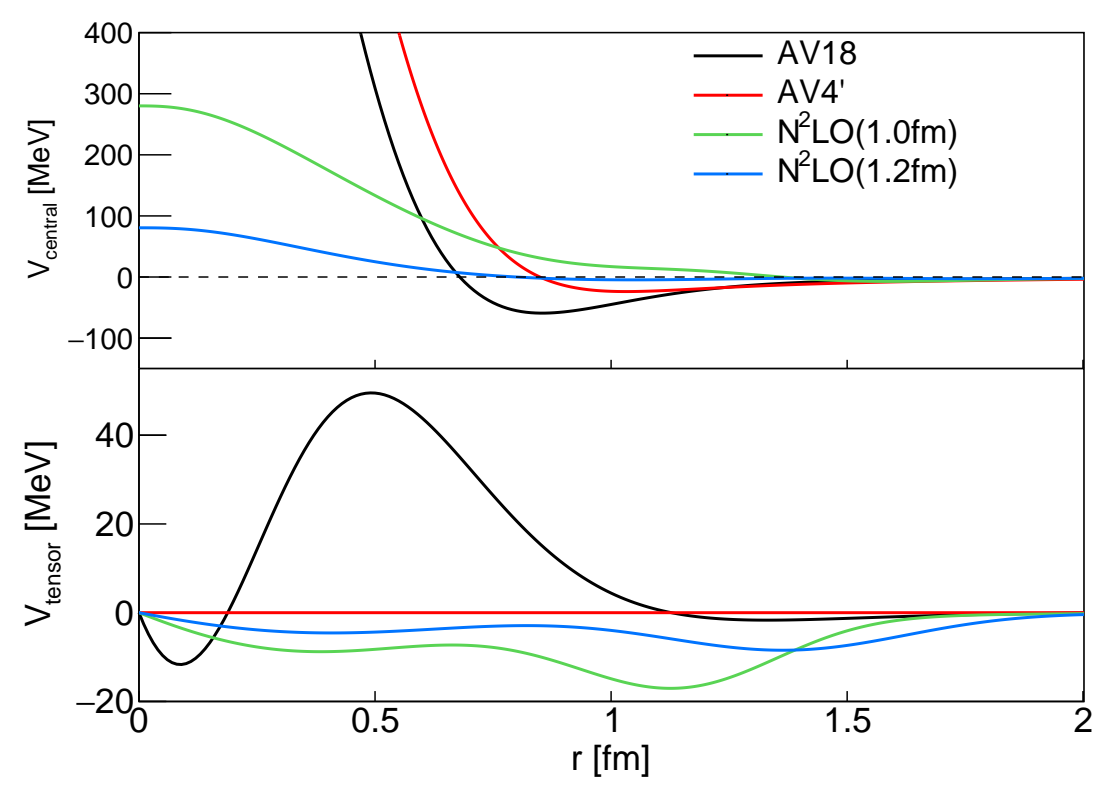

Figure 1-2: Central (top) and tensor (bottom) channels from four different $N N$ potentials as a function of nucleon-nucleon separation $r$. The black and red lines correspond to the phenomenological AV18 [4] and AV4' [5] respectively. The green and blue lines correspond to the $\chi \mathrm{EFT} \mathrm{N}{ }^{2} \mathrm{LO}$ with $1.0 \mathrm{fm}$ and $1.2 \mathrm{fm}$ short-distance cutoffs respectively [6, 7]. The AV4' potential is intentionally built without a tensor force. The short-distance behavior is predicted to be very different across different models.

$N N$ potentials need to be complemented with $3 N$ (three-nucleon) forces in order to accurately describe nuclear properties such as binding energies. The AV18 and 
AV4' potentials are typically supplemented with the Urbana-X (UX) and the central component of Urbana-IX ( $\left.\mathrm{UIX}_{\mathrm{c}}\right) 3 N$ forces respectively (AV18+UX, AV4' $\left.+\mathrm{UIX}_{\mathrm{c}}\right)$ [8, 9].

\section{$\chi$ EFT potentials}

Below the chiral symmetry-breaking scale $\left(\Lambda_{\chi} \sim 1 \mathrm{GeV}\right)$, the relevant degrees of freedom are nucleons and pseudoscalar mesons (rather than quarks and gluons). In this regime, the $N N$ interaction can be described as a systematic expansion of diagrams similar to that of Fig. 1-1, organized in powers of $Q / \Lambda_{\chi}$, where $Q$ refers to the momentum scale being considered. The Leading Order (LO) corresponds to all diagrams for which the amplitude is $\sim \mathcal{O}\left(Q / \Lambda_{\chi}\right)^{0}$. All the amplitudes corresponding to $\sim \mathcal{O}\left(Q / \Lambda_{\chi}\right)^{1}$ vanish due to parity and time-reversal symmetries. Thus, the Nextto-Leading Order $(\mathrm{NLO})$ corresponds to $\sim \mathcal{O}\left(Q / \Lambda_{\chi}\right)^{2}$, the Next-to-Next-to-Leading Order $\left(\mathrm{N}^{2} \mathrm{LO}\right)$ corresponds to $\sim \mathcal{O}\left(Q / \Lambda_{\chi}\right)^{3}$, etcetera. Table 1.1 shows the first few diagrams contributing to the chiral $N N$ interaction.

Table 1.1: $\chi$ EFT hierarchy of $N N$ forces. Solid and dashed lines represent nucleons and pions respectively. For more details and for the meaning of the different types of vertices see, e.g., [10].

\begin{tabular}{|c|c|c|c|}
\hline & & $N N$ & $3 N$ \\
\hline $\mathrm{LO}$ & $\mathcal{O}\left(Q / \Lambda_{\chi}\right)^{0}$ & & - \\
\hline NLO & $\mathcal{O}\left(Q / \Lambda_{\chi}\right)^{2}$ & & - \\
\hline $\mathrm{N}^{2} \mathrm{LO}$ & $\mathcal{O}\left(Q / \Lambda_{\chi}\right)^{3}$ & & \\
\hline
\end{tabular}

The most appealing features of chiral potentials are that 1) the diagrams are calculated based on the laws of the strong interaction (Quantum Chromo Dynamics, QCD), and that, 2) due to the hierarchical nature of the diagrammatical expansion, uncertainties can be systematically quantified, and results can be systematically improved based on the order to which the expansion has been carried out. The expansion 
is parametrized with low-energy constants that are determined from fits to scattering data. Fig. 1-2 shows two $\chi$ EFT potentials calculated to $\mathrm{N}^{2} \mathrm{LO}$ by Gezerlis, Tews and others [6, 7], with short-distance regulators (or cutoffs) at 1.0 and $1.2 \mathrm{fm}$. These regulators make the interactions "softer" (i.e. with a weaker short-distance repulsion and hence less high-momentum strength compared to phenomenological potentials). These potentials are complemented with the $E \mathbb{1}$ and $E \tau$ parametrizations of the $3 N$ forces [11]. For more details, see [10].

Another example of a chiral interaction used in this work is that of the Norfolk potentials denoted NV2+3-Xx* (e.g., NV2+3-Ia*), where X (= I or II) refers to the range of the phase-shift fit and $\mathrm{x}(=\mathrm{a}$ or $\mathrm{b})$ refers to the cutoffs used. The Norfolk potentials differ from the previously described $\mathrm{N}^{2} \mathrm{LO}$ potentials. For example, $\mathrm{NV} 2+3-\mathrm{Xx}^{*}$ explicitly include intermediate delta-isobars and their 2-body interaction includes contact terms up to fourth order $\left(\mathrm{N}^{3} \mathrm{LO}\right)$ in the chiral expansion. For additional details see, [12].

\subsection{Quantum Monte Carlo (QMC)}

The increase of computational power in recent decades enables the use of different numerical methods to solve the many-body Schrödinger equation:

$$
i \hbar \frac{\partial}{\partial t} \Psi_{A}=\hat{H} \Psi_{A},
$$

from first principles (ab initio) and obtain the ground state of the system [13]. Here, the nuclear wave function $\Psi_{A}$ has spatial, spin, and isospin degrees of freedom, and the Hamiltonian is given by:

$$
\begin{aligned}
\hat{H} & =\hat{T}+\hat{V}, \\
\hat{V} & =\sum_{i<j}^{A} V_{N N}(i, j)+\sum_{i<j<k}^{A} V_{3 N}(i, j, k),
\end{aligned}
$$


where $\hat{T}$ is the kinetic term, and the potential $\hat{V}$ has spatial, spin, and isospin operator dependences. $V_{N N}$ and $V_{3 N}$ can be any of the $N N$ potentials and $3 N$ forces described in the previous section. One class of these numerical methods is referred to as Quantum Monte Carlo (QMC), and includes techniques such as Variational Monte Carlo (VMC, which uses the variational principle to find the parameters of a trial wave function that minimize the ground state energy) [14], Diffusion Monte Carlo (DMC, which propagates a trial wave function in imaginary-time) [15], and others. Results can also be extrapolated by combining these techniques. Due to computational requirements, QMC calculations are currently mostly limited to light and medium $(A \leq 12)$ nuclei. Some heavier, closed-shell nuclei such as ${ }^{16} \mathrm{O}$ and ${ }^{40} \mathrm{Ca}$ can also be calculated making use of the symmetries of these systems (cluster expansion) [16].

The resulting QMC wave functions can be used to calculate quantities such as cross sections, momentum distributions, and coordinate densities. The 1-body momentum distribution describes the probability to find a nucleon in the nucleus with a given momentum $\vec{k}$. Similarly, the 2-body momentum distribution describes the probability to find two nucleons with relative momentum $\vec{q}$, and center-of-mass momentum $\vec{Q}$. Last, but not least, 2-body coordinate densities describe the probability of finding two nucleons with some relative separation $\vec{r}$ and distance from the center-of-mass of the nucleus $\vec{R}$.

There are several issues with current $N N$-interaction models [17]. Different potentials predict very different behaviors at short distance, as can be seen in Fig. 1-2. As a result, the short-distance/high-momentum component of the different nuclear quantities calculated based on QMC wave functions depend on the scheme (specific $N N$ model) and scale (the short-distance regulator or cutoff used within that model). At such small distances, Two-Nucleon Short-Range Correlations (SRCs) are expected to be the dominant component of the nuclear wave function and, consequently, studying SRCs can help us understand the $N N$ interaction (and vice versa). 


\subsection{Two-Nucleon Short-Range Correlations (SRCs)}

SRCs are described as pairs of nucleons that interact at short distances and, as a result of the interplay between the different channels of the $N N$ interaction, fly apart with high individual- and relative-momenta, and smaller center-of-mass momentum relative to the nuclear Fermi momentum $\left(k_{F} \approx 250 \mathrm{MeV} / c \approx 1.27 \mathrm{fm}^{-1}\right.$, [18]). For a detailed SRC review see, e.g., [19]. The study of SRCs also has implications for studies of nuclear charge radii [20], neutrino-less double-beta decay [21, 22, 23, 24, 25, 26], the partonic structure of nucleons bound in nuclei [19, 27, 28, 29, 30], neutron stars [31, 32, 33], and others.

In traditional nuclear physics, nucleons in the nucleus are described as individual particles interacting through the average potential created by the other $A-1$ nucleons. The eigenstates of these Mean-Field (MF) models predict a set of bound single-particle orbits. One of the most successful of these models is the Independent-Particle ShellModel (IPSM). While this single-particle description seems very crude given that the nucleus is very dense, these models describe many bulk properties of nuclei well. These models, however, fail to describe the short-distance and high-momentum components of the nuclear many-body wave function.

The importance of SRCs was introduced theoretically by R. Jastrow in 1955 [34]. He used variational techniques to examine the ground state properties of a stronglyinteracting many-body system. The nuclear wave function is parametrized by an anti-symmetrized product of single-particle wave functions. In the hard-sphere limit, which approximates the repulsive core of the $N N$ interaction, the ground state energy of this system increases without limit. This problem can be remedied through the introduction of functions that depend on the $N N$ separation $r_{i j}$ such that: $\psi \sim$ $\prod_{i<j=1}^{N} \mathbb{F}\left(r_{i j}\right)$. These "correlation" functions, which will be reviewed in more detail in chapter 4 of this thesis, are defined so that $\mathbb{F}\left(r_{i j}\right)$ vanishes for $r<r_{0}$ and approaches unity for $r \gg r_{0}$ (where $r_{0}$ refers to the range of the strong interaction). This was the first allusion to the importance of SRCs in nuclei.

Experimentally, ground-state charge-density distributions were calculated using 
semi-realistic $N N$ potentials and Mean-Field wave functions that did not include SRCs and compared to electron scattering data [35]. The calculated densities overestimated the interior density of the nucleus by up to $20 \%$. L. Lapikas compared IPSM calculations to electron-induced knock-out of protons from different nuclear shells [36]. While these calculations described the shape of momentum distributions for nucleons in the valence and next deeper sub-shells, the spectroscopic strengths for valence orbitals were much lower than expected (see Fig. 1-3). Since Mean-Field models describe nucleons as individual particles, the next step in the potential expansion corresponds to two-nucleon correlations. Calculations including the effect of longrange correlations still disagree with experimental observations [37]. The additional inclusion of SRCs is needed to fully account for the spectroscopic strengths. Within the theoretical framework used to extract the spectroscopic factors, this leads to an effective description of the nucleus that has two main regions in momentum space: 1) a Mean-Field region below $k_{F}$ dominated by single-nucleon behavior and longrange correlations, and containing $\approx 80 \%$ of all nucleons, and 2) an SRC-dominated high-momentum tail above $k_{F}$ that contains $\approx 20 \%$ of all nucleons.

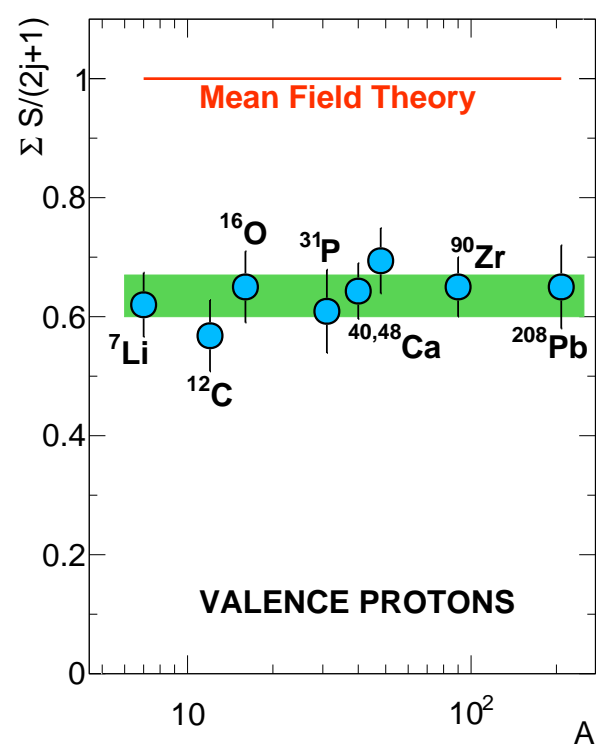

Figure 1-3: Summed spectroscopic strengths for valence orbitals from electroninduced proton-knockout cross sections compared to Mean-Field theory expectations 36 . 
Inclusive QE electron-scattering measurements in high- $Q^{2}$ and $x_{B}>1$ kinematics have been traditionally used to study SRCs. Here, $x_{B}$ is the fraction of the nucleon longitudinal momentum carried by the struck quark $2^{2}$ and $Q^{2}$ is the magnitude squared of the four-momentum transferred by the probe in the scattering process. See chapter 2 for a formal definition of these variables. In these measurements, it is observed that the (per-nucleon) cross-section ratios between two nuclei $A_{1}$ and $A_{2}$ :

$$
R\left(A_{1}, A_{2}\right) \equiv \frac{\sigma\left(A_{1}, x_{B}, Q^{2}\right) / A_{1}}{\sigma\left(A_{2}, x_{B}, Q^{2}\right) / A_{2}},
$$

scale for $x_{B}>1.5$ (see Fig. 1-4). That is, due to the scale separation that exists between the strong interaction between the two nucleons in the SRC pair, and their weaker interaction with the rest of the system, when scattering from SRC nucleons the cross section in different nuclei is approximately the same up to a constant factor $R\left(A_{1}, A_{2}\right)$. This factor is interpreted as the relative abundance of SRCs in nucleus $A_{1}$ relative to $A_{2}$. This was observed for the first time at SLAC [38], where ratios for different nuclei relative to deuteron, $a_{2}(A) \equiv R(A, \mathrm{~d})$ were studied. These measurements were subsequently extended at JLab, where $a_{3}(A) \equiv R\left(A,{ }^{3} \mathrm{He}\right)$ was also studied [30, 39, 40, 41].

Semi-inclusive electron-induced QE proton-knockout experiments, in which both the scattered electron and knocked-out proton are detected, provide additional information with respect to inclusive experiments. Measurements in which the electronscattering process can breakup the ${ }^{3} \mathrm{He}$ nucleus into a proton and a deuteron (2body breakup) or two protons and a neutron (3-body breakup) carried out at $Q^{2}=$ $1.5\left(\mathrm{GeV} / c^{2}\right)^{2}$ and $x_{B}=1$ in Hall A of JLab found good agreement with Plane-Wave Impulse Approximation (PWIA) calculations up to missing momenta $2^{3}$ approximately equal to the typical nucleus Fermi momentum [42, 44]. However, at higher missing momenta, the measured and PWIA-based distributions disagreed by up to $400 \%$. Additional calculations that included non-QE reaction mechanisms were needed to

\footnotetext{
${ }^{2}$ In a nucleus of mass number $A, x_{B}$ can take any value from 0 to $A$, since there can be up to $A$ nucleons present from which the knocked-out nucleon can take momentum.

${ }^{3}$ In the PWIA, the missing momentum equals the initial nucleon momentum. See chapter 2 for details.
} 


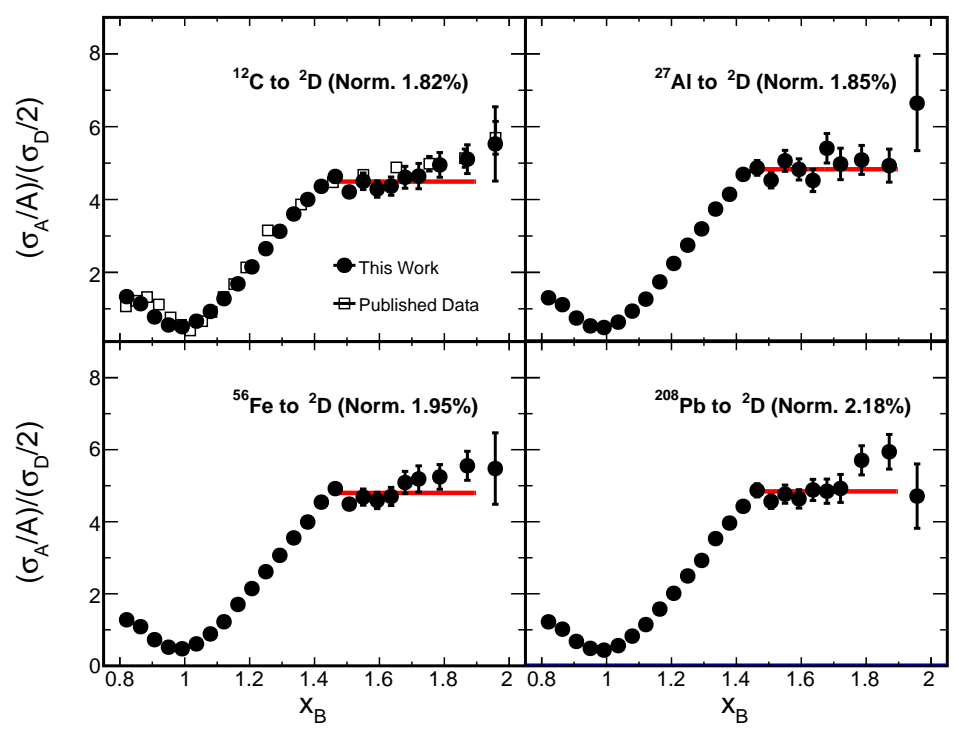

Figure 1-4: Inclusive $\left(e, e^{\prime}\right)$ per-nucleon cross-section ratios for ${ }^{12} \mathrm{C},{ }^{27} \mathrm{Al},{ }^{56} \mathrm{Fe}$, and ${ }^{208} \mathrm{~Pb}$ to deuteron as a function of $x_{B}$ from [30]. At $x_{B}>1.5$ the cross sections scale, as expected from scattering off SRC nucleons. See text for details.

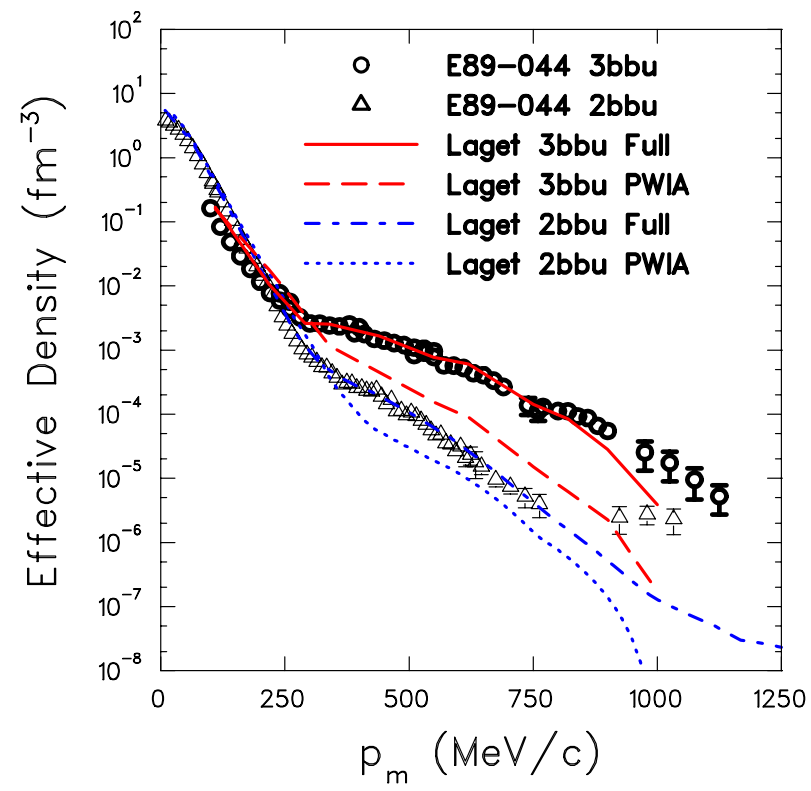

Figure 1-5: ${ }^{3} \mathrm{He}\left(e, e^{\prime} p\right)$ reduced cross section as a function of missing momentum from [42]. The $\bigcirc$ and $\triangle$ markers correspond to the measured 3- and 2-body breakup reactions respectively, and are compared to calculations from [43. The red dashed (- - ) and blue dotted (-...-) lines correspond to PWIA calculations. The red solid and blue dash-dotted (-- ) lines correspond to full calculations that include the effect of non-QE reaction mechanisms. See text and chapter 2 for more details. 
explain the observed discrepancy. See Fig. 1-5. Additional semi-inclusive SRC measurements from JLab have been carried out in deuteron [45] and ${ }^{12} \mathrm{C}$ [46]. Since this type of measurement is the focus of this thesis, details on the reaction and discussions about these previous studies will be presented in chapters 2 and 3 .

In exclusive or triple-coincidence experiments, information from the two nucleons from the SRC pair can be obtained. For instance, the measurement of the protoninduced two-nucleon knockout ${ }^{12} \mathrm{C}(p, 2 p n)$ reaction performed at Brookhaven National Laboratory by the Eva collaboration allowed the determination of the angle between the knocked-out proton and the recoil neutron, showing that above $k_{F}$, where SRCs are expected to dominate, the two nucleons are ejected from the nucleus preferentially in back-to-back configurations. [47, 48, 49]. See Fig. 1-6 (left). In this analysis, the width of the center-of-mass momentum distribution of SRC pairs was determined to be $\sigma_{c . m}^{12} \mathrm{C}=143 \pm 17 \mathrm{MeV} / c$. This study was expanded by [50] using (e, $\left.e^{\prime} p p\right)$ data from Hall $\mathrm{B}$ of JLab on ${ }^{12} \mathrm{C},{ }^{27} \mathrm{Al},{ }^{56} \mathrm{Fe}$, and ${ }^{208} \mathrm{~Pb}$. It was found that the pair center-ofmass motion in these nuclei can be described by a three-dimensional Gaussian with a width ranging from 140 to $170 \mathrm{MeV} / c$, which is approximately consistent with the sum of two Mean-Field nucleon momenta. See Fig. 1-6 (right). The ${ }^{12} \mathrm{C}$ result was consistent with the equivalent result from [47] and [51]. The extracted widths are also consistent with calculations from [52, 53, 54, 55], which supports the theoretical picture of SRC pair formation from temporal fluctuations of Mean-Field nucleons.

The analysis from [49] also showed that the removal of a proton from the nucleus with initial momentum between $275-550 \mathrm{MeV} / c$ was $92_{-18}^{+8} \%$ of the time accompanied by the emission of an approximately back-to-back neutron, which indicates that the probabilities to encounter $p p$ or $n n$ SRC pairs in the nucleus are at least a factor of six smaller than that of $p n$. This result is known as $n p$ dominance of SRCs and has been studied and confirmed in a variety of analyses. Fig. 1-7 (left) shows the SRC pair fractions as a function of missing momentum from the previously mentioned analysis and also from a measurement from Hall A of JLab [51, 57]. They found that there are approximately 20 times more $n p$ pairs than $p p$ or $n n$ pairs [47, 60]. These $n p$-dominance observations on ${ }^{12} \mathrm{C}$ were extended to ${ }^{27} \mathrm{Al},{ }^{56} \mathrm{Fe}$, and ${ }^{208} \mathrm{~Pb}$ by 

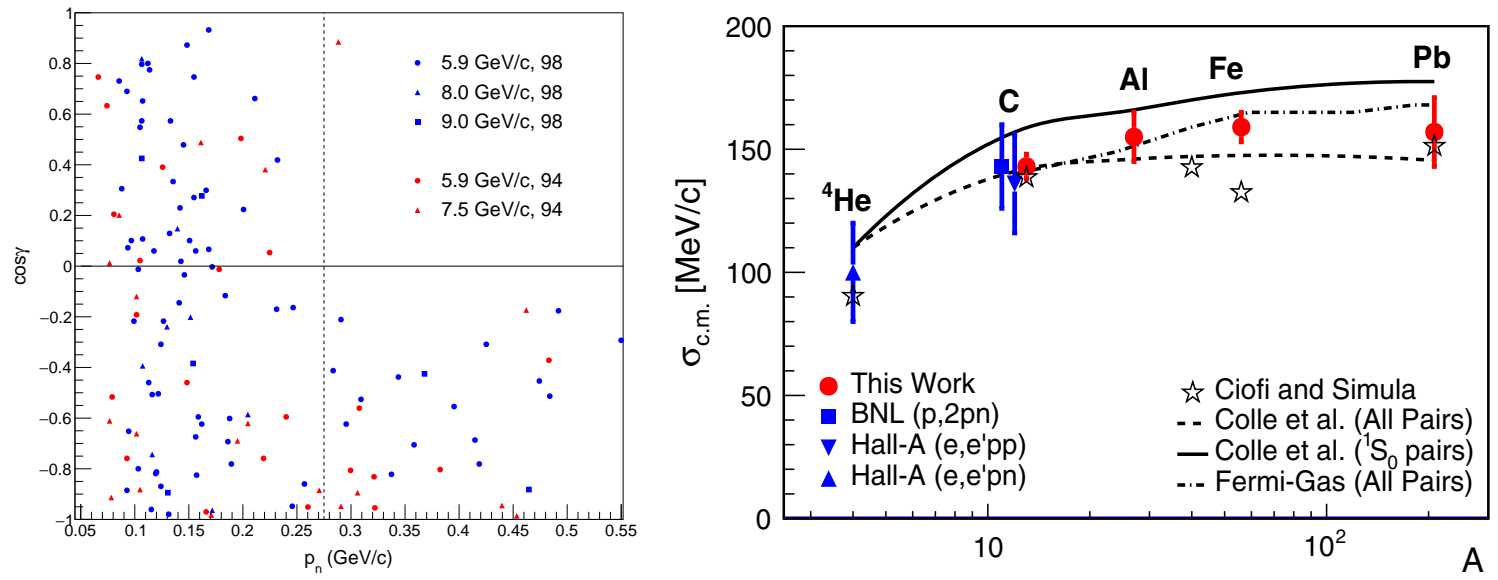

Figure 1-6: Center-of-mass momentum distribution of SRC pairs. Left: cosine of the angle $\gamma$ between the struck proton and the recoil neutron, as a function of recoilneutron momentum $p_{n}$. Above the Fermi momentum, where SRCs dominate, an angular correlation between the two nucleons is observed [49]. Right: width of the center-of-mass momentum distribution of SRCs for different nuclei [50]. The red (-) markers correspond to the result obtained in this analysis. The blue markers corre-

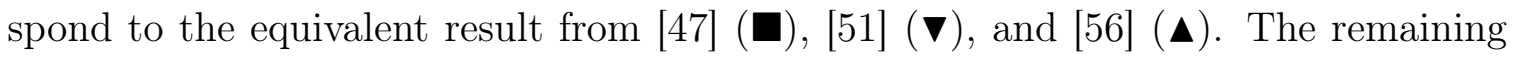
markers and lines correspond to theoretical calculations from [52, 53, 54, 55].
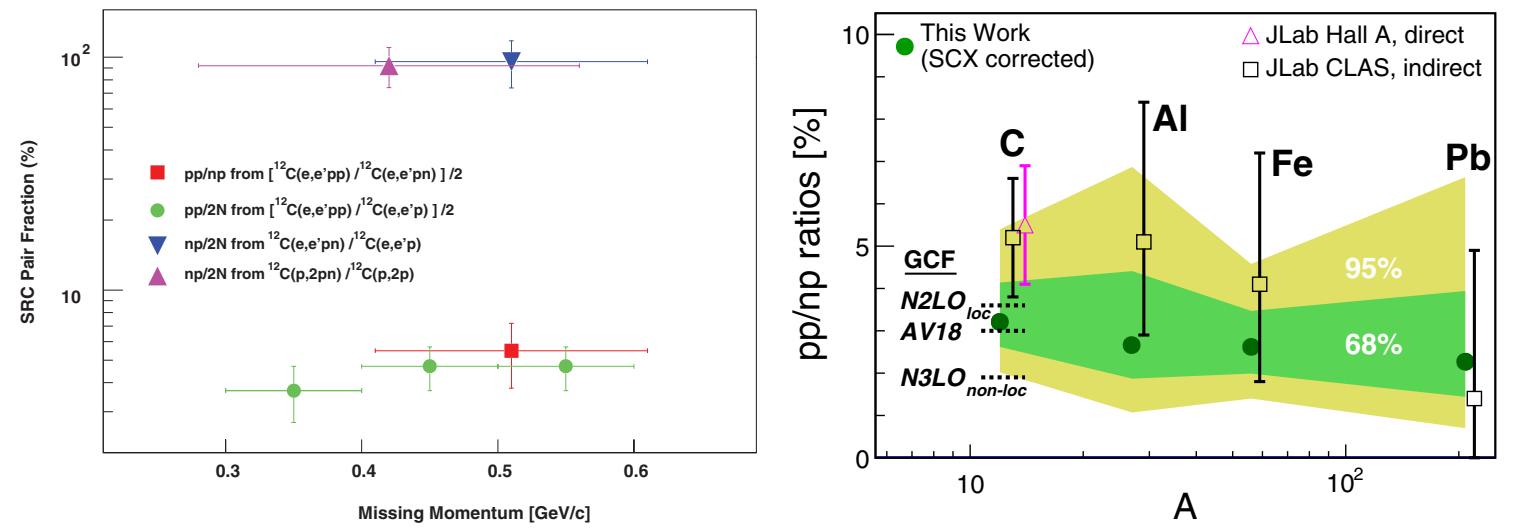

Figure 1-7: $n p$ dominance of SRCs. Left: SRC pair fraction in ${ }^{12} \mathrm{C}$ [57]. The red $(\boldsymbol{\square})$, green $(\boldsymbol{\bullet})$, and blue $(\boldsymbol{\nabla})$ markers correspond to the $p p / n p, p p / 2 N$, and $n p / 2 N$ fractions respectively. The purple marker $(\boldsymbol{\Lambda})$ corresponds to the $n p / 2 N$ result from [49]. Right: $p p / n p$ fraction in ${ }^{12} \mathrm{C},{ }^{27} \mathrm{Al},{ }^{56} \mathrm{Fe}$, and ${ }^{208} \mathrm{~Pb}$ from [58] shown as green (-) markers. The green and yellow bands correspond to the $68 \%$ and $95 \%$ confidence limits respectively. The markers $\triangle$ and $\square$ correspond to the equivalent results from [57] and [59] respectively. The horizontal dashed lines correspond to the ${ }^{12} \mathrm{C}$ prediction from the Generalized Contact Formalism (GCF) (see chapter 4) using different $N N$ potentials. 
[59] by analyzing the $\left(e, e^{\prime} p\right)$ and $\left(e, e^{\prime} p p\right)$ reactions, and later bolstered by [58] by analyzing the $\left(e, e^{\prime} p n\right)$ and $\left(e, e^{\prime} p p\right)$ reactions from data measured in Hall B of JLab. See Fig. 1-7 (right).

The interpretation of this result is that the experimentally-probed momentum range (300 $\lesssim k \lesssim 600 \mathrm{MeV} / c$ ) corresponds to the region of the $N N$ potential around the point in which the central channel crosses a minimum and the tensor force takes over and governs the interaction [61, 62] (see Fig. 1-2). The tensor force only couples to spin-1 $N N$ pairs, and since short-distance $p p$ and $n n$ pairs are predominantly spin0 (due to the Pauli exclusion principle), pairs in this region are mostly $n p$. This causes the $p p$ and $n n$ densities as a function of relative momentum to have a dip around $380 \mathrm{MeV} / c$, and this dip is not present in the total $p n$ density. See Fig. 1-8 (right). As nucleons interact at even shorter distances, the scalar force should take over the interaction (see top panel in Fig. 1-2), and spin-0 contributions are expected to increase. This statement is supported by studies of the $\left(e, e^{\prime} p\right),\left(e, e^{\prime} p p\right)$, and $\left(e, e^{\prime} p n\right)$ reactions presented in [56, 63, 64].

This tensor-dominated momentum regime is further supported by the result from a ${ }^{3} \mathrm{He}\left(e, e^{\prime} p p\right) n$ measurement carried out in Hall B of JLab [65]. In this study, the relative and total momentum distributions of $p p$ and $p n$ pairs in ${ }^{3} \mathrm{He}$ were measured by detecting events in which the electron scattered off an uncorrelated nucleon, and the two (spectator) nucleons from the SRC pair were emitted from the nucleus with high momenta. Fig. 1-8 (left) shows the ratio of $p p / p n$ (spectator) SRC pairs integrated over relative momentum and as a function of center-of-mass momentum of the SRC pair. As center-of-mass momentum of the pair is increased, the dip in the $p p$ distribution gets smeared, and the relative fraction of $p p$ to $p n$ pairs increases. This is shown in Fig. 1-8 (right), where ${ }^{3} \mathrm{He}$ 2-body momentum distributions $F_{N N}(q, Q)$ for different values of $Q$ from [66] are shown. The dip in the $p p$ distribution gets filled as $Q$ increases.

Lastly, [60] studied the double ratios of the number of $\left(e, e^{\prime} N\right)$ high-momentum (SRC) nucleon events to low-momentum (Mean-Field) nucleon events for nucleus $A$ $\left({ }^{27} \mathrm{Al},{ }^{56} \mathrm{Fe}\right.$, and $\left.{ }^{208} \mathrm{~Pb}\right)$ relative to ${ }^{12} \mathrm{C}$ : 

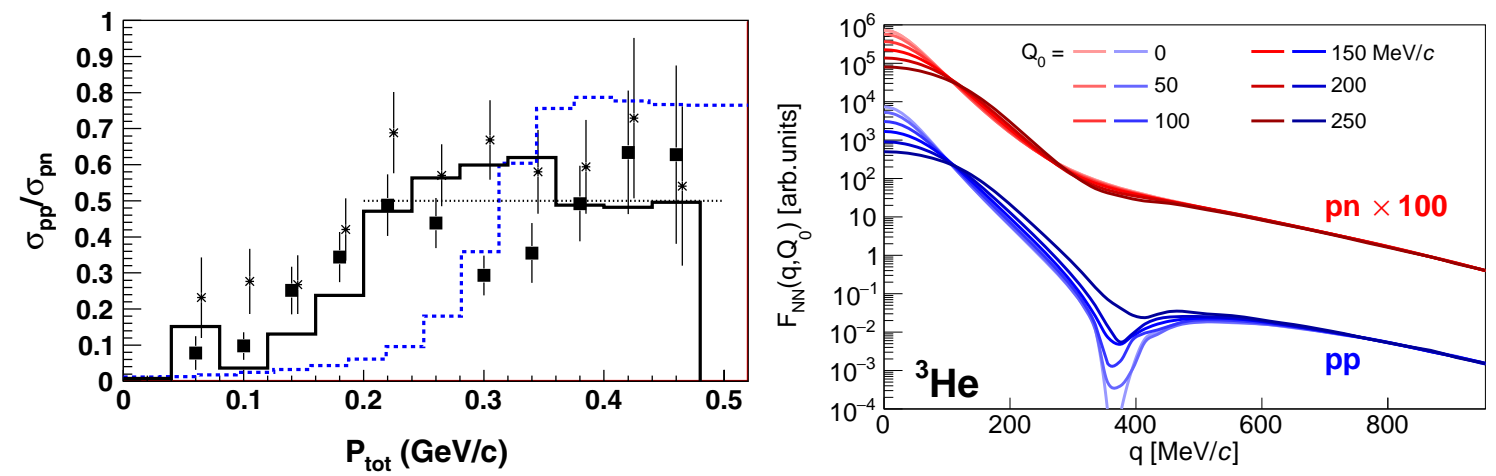

Figure 1-8: Effect of pair center-of-mass momentum on relative $p p$-to- $p n$ abundance. Left: ratio of $p p / p n$ (spectator) SRC pairs from ${ }^{3} \mathrm{He}\left(e, e^{\prime} p p\right) n$ measurements integrated over relative momentum between $0.3-0.5 \mathrm{GeV} / c(\mathbf{a})$ and between $0.4-0.6$ $\mathrm{GeV} / c(*)$ as a function of center-of-mass momentum of the pair $P_{t o t}$ [65]. The black solid and blue dashed curves correspond respectively to a 1-body and a $p p / p n$ boundstate momentum distribution calculations from [67]. The dotted line at 0.5 shows the simple pair counting result. The data and the 1-body calculation have been multiplied by 1.5 to approximately account for the ratio of the average electron-proton and electron-neutron elementary cross sections. Right: QMC calculations of the ${ }^{3} \mathrm{He}$ 2-body momentum distribution $F_{N N}(q, Q)$ as a function of relative momentum $q$ for different values of center-of-mass momentum $Q \equiv P_{t o t}$. The distributions have been arbitrarily scaled to emphasize their shapes.

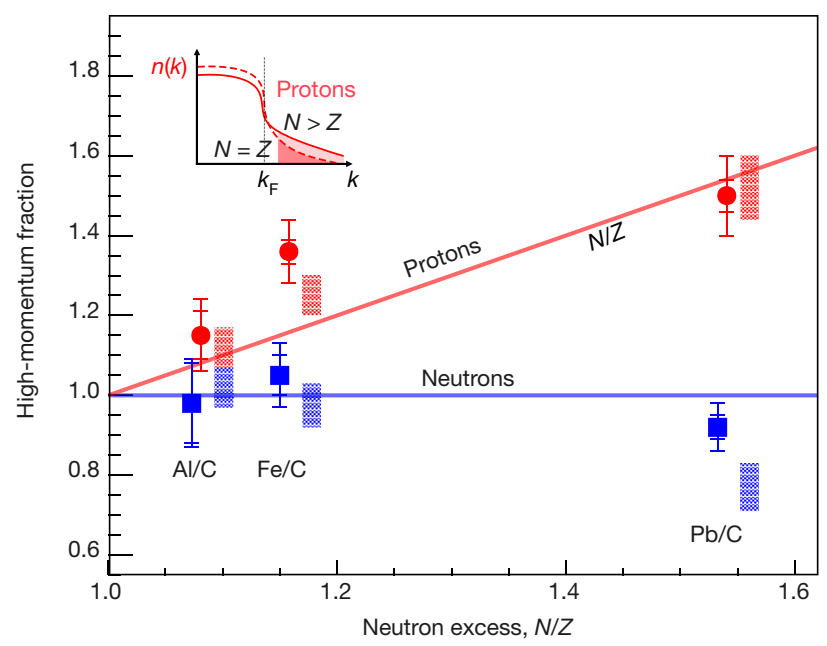

Figure 1-9: Fraction of high-momentum nucleons in nucleus $A$ relative to carbon (see equation 1.5) as a function of neutron excess [60]. The red ( $)$ and blue $(\boldsymbol{\square})$ markers correspond to protons and neutrons respectively. The red and blue shaded rectangles correspond to the equivalent quantity calculated phenomenologically using an $n p$ dominance model. The red and blue lines correspond to $N / Z$ and 1 respectively, and are drawn to guide the eye. As the number of neutrons increases in the nucleus, the fraction of high-momentum neutrons stays approximately equal, while the fraction of high-momentum protons increases. 


$$
\frac{A\left(e, e^{\prime} N\right)_{\mathrm{high}} /\left.A\left(e, e^{\prime} N\right)\right|_{\text {low }}}{{ }^{12} C\left(e, e^{\prime} N\right)_{\mathrm{high}} /{ }^{12} C\left(e, e^{\prime} N\right)_{\text {low }}},
$$

which describes the increase in the fraction of high-momentum protons and neutrons in each considered asymmetric nucleus relative to carbon. It was found that the fraction of high-initial-momentum protons increases by $\sim 50 \%$ from carbon to lead, while the fraction of high-initial-momentum neutrons decreases by $\sim 10 \%$. See Fig. 1 9. This result implies that, on average, protons move faster than neutrons in neutronrich nuclei.

\subsection{Scope of this work}

The main objective of this work is to further the current knowledge on the $N N$ interaction and SRCs by putting QMC calculations to test both from an experimental and a phenomenological standpoints. This thesis has two primary parts. In the first part, I describe how we can use measurements of electron-scattering proton knockout from light nuclei to constrain the $N N$ interaction. Chapter 2 presents the theoretical description of the QE electron-induced proton-knockout reaction $\left(e, e^{\prime} p\right)$ in $A=3$ nuclei. Chapter 3 describes the experimental extraction of ${ }^{3} \mathrm{He}$ and ${ }^{3} \mathrm{H}\left(e, e^{\prime} p\right)$ absolute cross sections and cross section ratios as a function of missing momentum from an experiment carried out at JLab, and compares them to a series of calculations using different models of the $N N$ potential in the momentum range $40-550 \mathrm{MeV} / c$. This momentum range covers the transition from single-particle (low momentum) behavior to two-particle SRC (high momentum) behavior.

Since traditional nuclear-structure effective models like the IPSM fail to describe the high-momentum and short-distance states in nuclei, effective models which include SRCs are essential to fully describe the nuclear ground state for nuclei for

which "exact" QMC calculations are not feasible. Chapter 4 presents the Generalized Contact Formalism (GCF) as an effective model to describe SRCs in nuclei. I then use the GCF together with several $N N$ models to study which SRC properties are scale-and-scheme dependent, and which are independent. Additionally, a list of appli- 
cations for the GCF is presented, and a GCF-based extraction of nuclear correlation functions is presented.

Throughout this thesis, I will present equations in natural units (i.e. $c=\hbar=1$ ).

\subsubsection{A word on notation changes}

Chapters 3 and 4 of this thesis are focused on experimental and phenomenological studies respectively. To be consistent with the nomenclature used in these fields, the notation between chapters 3 and 4 changes slightly.

Chapter 3 uses $q$ and $Q$ for the electron-scattering momentum transferred by the virtual photon. In chapter $4, q$ and $Q$ are the relative and center-of-mass momenta respectively. The single-particle momentum is written as $p$ in chapter 3 , and as $k$ in chapter 4. Lastly, momentum and energy units in chapter 3 are given in powers of electron-Volts: $\mathrm{MeV}, \mathrm{GeV}, \ldots$, whereas in chapter $4 . \mathrm{fm}^{-1}$ is used (the conversion between these units is done by multiplying or dividing by $\hbar c \approx 197.3 \mathrm{MeV} \cdot \mathrm{fm}$ ). 


\section{Chapter 2}

\section{Quasi-Elastic Electron Scattering off Light-Nuclei}

\section{$2.1 \quad$ Introduction}

The structure of small objects (e.g. atomic arrays, atoms, nuclei, nucleons, ...) can be studied by examining how a probe (of known properties) scatters off them. In nuclear structure studies, the electron is commonly used as a probe due to its lack of internal structure and its very-well understood electromagnetic interaction. Electrons scatter from nuclei by exchanging a single virtual photon. By tuning the resolving wavelength of the virtual photon, $\lambda_{\gamma}$ (determined by how much momentum it transfers to the probed object) one can learn about properties of the nucleus at different scales. Fig. 21 shows a cartoon illustrating the overall features of the electron-nucleus cross section as a function of transfer energy. At the low end of the spectrum $\left(\lambda_{\gamma} \sim r_{A}\right.$, the nucleus radius) the virtual photon emitted by the electron probe interacts coherently with the nucleus as a whole (elastic scattering) providing a global description of this system. At the high end of the spectrum $\left(\lambda_{\gamma} \ll r_{N}\right.$, the nucleon radius) the virtual photon elastically interacts with one of the quarks forming the nucleons in the nucleus (Deep Inelastic Scattering or DIS), allowing us to study the nucleon internal structure. In the intermediate region, the virtual photon can excite the nucleus or its nucleons into resonant states $\left(\Delta, \mathrm{N}^{*}, \ldots\right)$. Finally, when $\lambda_{\gamma} \sim r_{N}$, the virtual photon can interact 
with a (quasi-free) nucleon bound in the nuclear medium, knocking it out of the nucleus without breaking it (quasi-elastic, QE scattering), providing a description of the nucleus internal structure. This last process will be the focus of this chapter and the next one.

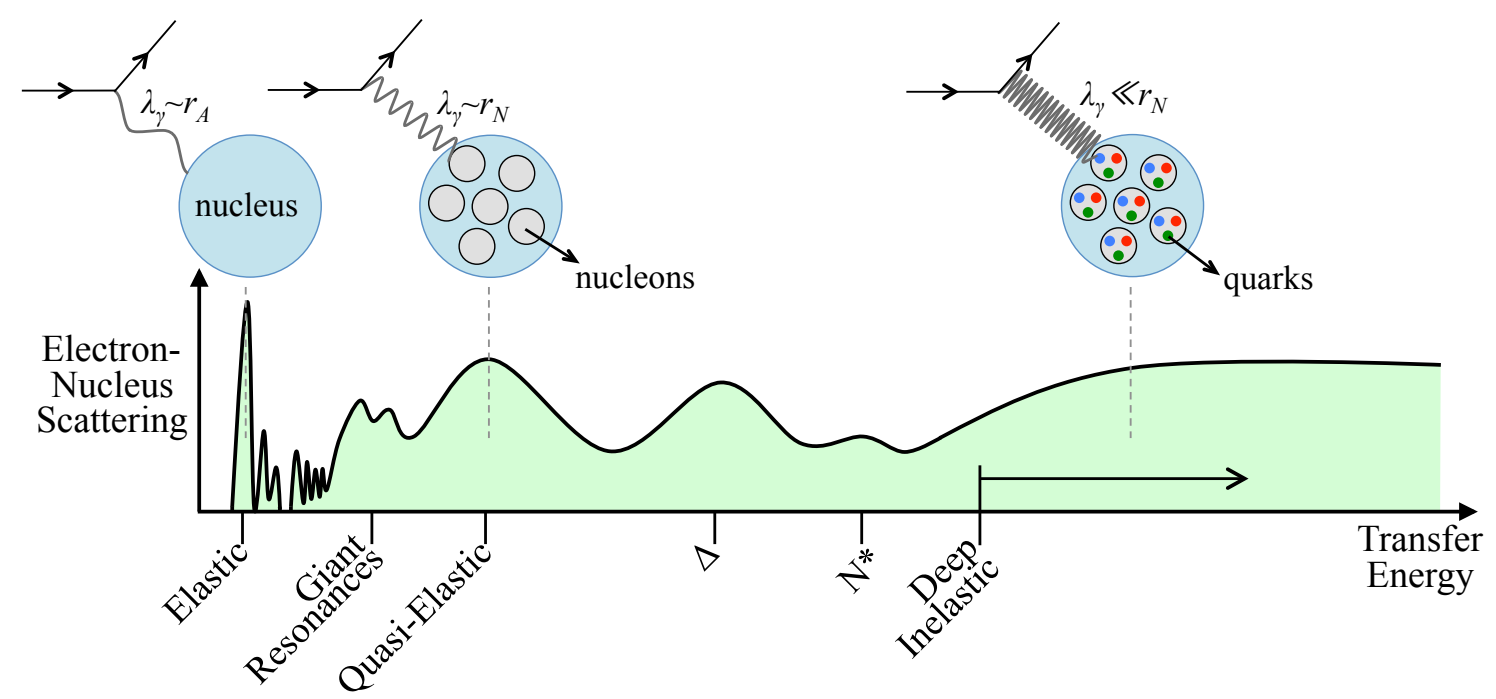

Figure 2-1: Electron-nucleus scattering cross section vs. transfer energy $(\omega)$ at constant $Q^{2}=\vec{q}^{2}-\omega^{2}$, where $q$ is the transfer momentum. As the virtual photon wavelength is decreased, smaller structures in the nucleus can be resolved.

\subsection{Quasi-elastic $\left(e, e^{\prime} p\right)$ scattering}

In quasi-elastic fixed-target electron-scattering measurements, electrons of momentum $p_{e}^{\mu}=\left(E_{e}, \vec{p}_{e}\right)$ scatter from a nuclear target $A$ of mass $m_{A}$ and momentum $p_{t a r}^{\mu}=$ $\left(m_{A}, \overrightarrow{0}\right)$ into a final state with momentum $p_{e^{\prime}}^{\mu}=\left(E_{e^{\prime}}, \vec{p}_{e^{\prime}}\right)$. In this process, a virtual $]^{1}$ photon of momentum $q^{\mu}=p_{e}^{\mu}-p_{e^{\prime}}^{\mu}=(\omega, \vec{q})$ and magnitude squared $Q^{2} \equiv-q^{\mu} \cdot q_{\mu}=$ $\vec{q}^{2}-\omega^{2}$ is emitted. As a result of the interaction with the virtual photon, a proton gets knocked out of the nucleus with momentum $p_{p}^{\mu}=\left(E_{p}, \vec{p}_{p}\right)$, and the $A-1$ system recoils with momentum $p_{\text {recoil }}^{\mu}$. See Fig. 2-2. The plane defined by the incoming and scattered electron vectors is called scattering plane, and the plane defined by

\footnotetext{
${ }^{1}$ A particle is denoted virtual if it is off its mass shell, meaning that it does not satisfy the energy-momentum relation $E^{2}=p^{2}+m^{2}$, where $E, p$, and $m$ are the particle energy, momentum, and mass respectively.
} 
the virtual photon and knocked-out proton vectors is called the reaction plane. The angle between these planes is denoted $\phi . \theta_{e^{\prime}}$ is the electron scattering angle. $\theta_{p q}$ and $\theta_{r q}$ are the angles between the virtual-photon vector and the knocked-out proton and recoiling system respectively.

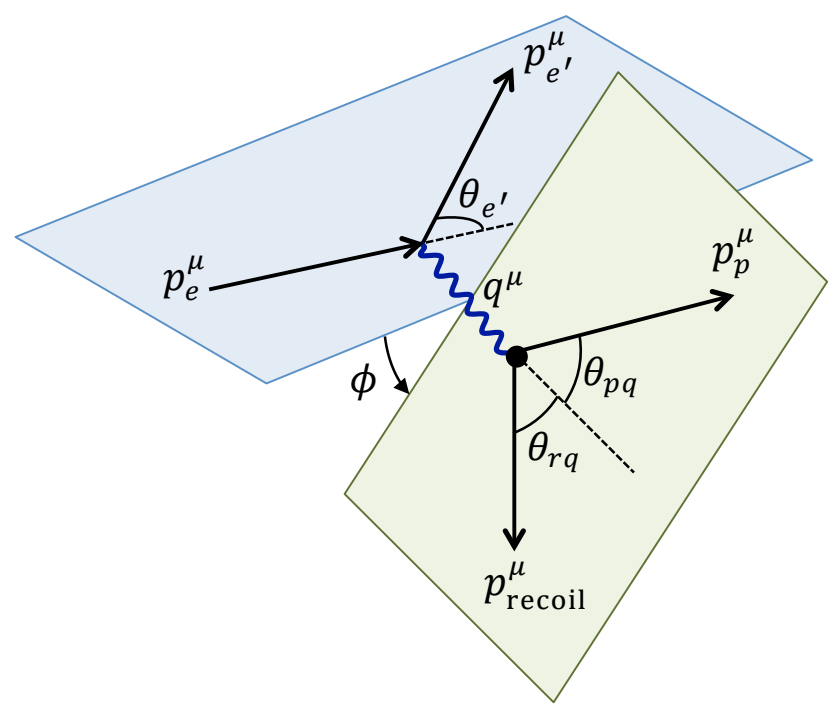

Figure 2-2: Diagram for $\left(e, e^{\prime} p\right)$ reaction kinematics. $p_{e}^{\mu}, p_{e^{\prime}}^{\mu}, p_{q}^{\mu}, p_{p}^{\mu}$, and $p_{\text {recoil }}^{\mu}$ are the Lorentz vectors for the incoming electron, scattered electron, virtual photon, knockedout proton, and $A-1$ recoiling system respectively. The blue area corresponds to the scattering plane, defined by the incoming and scattered electron vectors. The green area corresponds to the reaction plane, defined by the virtual photon and knocked-out proton vectors.

If only the scattered electron is measured, then it is called inclusive-scattering, and labeled $\left(e, e^{\prime}\right)$ (for an $\left(e, e^{\prime}\right)$ review see, e.g., [68]). If the momentum of the knockedout proton is measured in coincidence with that of the scattered electron $\left(\left(e, e^{\prime} p\right)\right)$, additional quantities, such as the missing (or undetected) momentum and energy, can be reconstructed:

$$
\begin{aligned}
\vec{p}_{\text {miss }} & =\vec{p}_{p}-\vec{q} \\
E_{\text {miss }} & =\omega-T_{p}-T_{A-1}
\end{aligned}
$$

where $T_{p}=E_{p}-m_{p}$ is the knocked-out proton kinetic energy, and $T_{A-1}$ is the recon- 
structed kinetic energy of the recoiling $A-1$ system, and can be written as:

$$
T_{A-1}=\left(\omega+m_{A}-E_{p}\right)-\sqrt{\left(\omega+m_{A}-E_{p}\right)^{2}-\left|\vec{p}_{m i s s}\right|^{2}} .
$$

\section{$\left(e, e^{\prime} p\right)$ Cross Section and the Plane-Wave Impulse Approximation}

The cross section for electron-induced proton knockout $\left(e, e^{\prime} p\right)$ scattering in the onephoton-exchange approximation (see, e.g., [69]) can be written as:

$$
\frac{d^{6} \sigma}{d \omega d E_{p} d \Omega_{e} d \Omega_{p}}=\sigma_{\mathrm{Mott}} \hat{l}^{\mu \nu} W_{\mu \nu}^{A}
$$

where $\sigma_{\text {Mott }}$ is the Mott cross section, and $\hat{l}_{\mu \nu}$ and $W_{\mu \nu}^{A}$ are the leptonic and nuclearresponse (hadronic) tensors respectively. $\Omega_{e}$ and $\Omega_{p}$ correspond to the solid angles for the scattered electron and knocked-out proton respectively. While the leptonic tensor is well understood, the hadronic tensor cannot be calculated rigorously from first principles for many-body systems without making approximations because of QCD having strong coupling in this regime.

A commonly used model in scattering calculations is the Plane-Wave Impulse Approximation (PWIA). The main assumptions of the PWIA are that: 1) the initial and final state particle wave functions can be described using plane waves $\left(\sim e^{i \vec{k} \cdot \vec{r}}\right)$, and 2) the interaction occurs over a very short time scale with respect to nuclear dynamics (impulse approximation). In PWIA, the nuclear response functions $\left(W_{\mu \nu}^{A}\right)$ are written in terms of nucleon response functions and, consequently, the cross section from equation 2.4 factorizes into [70]:

$$
\left.\frac{d^{6} \sigma}{d \omega d E_{p} d \Omega_{e} d \Omega_{p}}\right|_{\mathrm{PWIA}}=K \sigma_{e p} S\left(\left|\vec{p}_{i}\right|, E_{i}\right),
$$

where $K=E_{p}\left|\vec{p}_{p}\right|$ is a kinematical factor, $\sigma_{e p}$ is the elementary cross section for an electron scattering from a bound proton [71], and $S\left(\left|\vec{p}_{i}\right|, E_{i}\right)$ is the spectral function, the probability to find a nucleon in the nucleus with momentum $\left|\vec{p}_{i}\right|$ and removal energy $E_{i}$. That is, in PWIA, the $\left(e, e^{\prime} p\right)$ cross section factorizes into a term that 
describes the interaction of the electron with the bound proton $\left(\sigma_{e p}\right)$, and a term that describes the configuration of that proton inside the nucleus $\left(S\left(\left|\vec{p}_{i}\right|, E_{i}\right)\right)$. The integral of the spectral function over removal energy corresponds to the 1-body momentum distribution (see section 1.2 ):

$$
n\left(\left|\vec{p}_{i}\right|\right)=\int_{0}^{\infty} S\left(\left|\vec{p}_{i}\right|, E_{i}\right) d E_{i}
$$

The diagrams describing the PWIA processes of interest are shown in Fig. 2-3. In diagram a), the virtual photon interacts with a single nucleon, knocking it out of the nucleus. Additionally, in diagram b), the two spectator (unstruck) nucleons can rescatter between themselves $\left(\mathrm{FSI}_{23}\right)$. As the struck nucleons from these diagrams leave the nuclear medium, they do not reinteract. Under these assumptions, the missing momentum and energy are equal to the initial nucleon momentum and removal energy respectively $\left(\vec{p}_{\text {miss }} \equiv \vec{p}_{i}, E_{\text {miss }} \equiv E_{i}\right)$. Consequently, interpreting an $\left(e, e^{\prime} p\right)$ cross section measurement through the PWIA allows us to access the spectral function 2

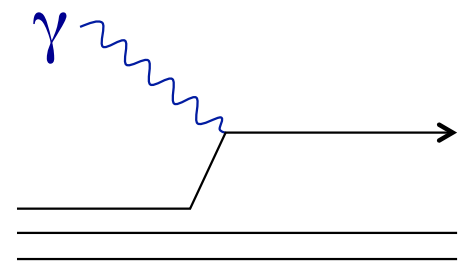

a) PWIA

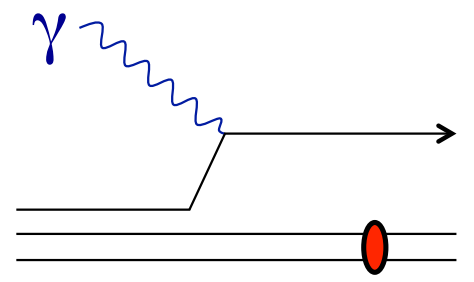

b) $\mathrm{FSI}_{23}$

Figure 2-3: Reaction mechanisms of interest: a) PWIA b) PWIA including the continuum final-state interaction between the two unstruck nucleons $\left(\mathrm{FSI}_{23}\right)$.

\subsubsection{Quasi-elastic $\left(e, e^{\prime} p\right)$ scattering off $A=3$ nuclei}

The $A=3$ system (namely the mirror nuclei ${ }^{3} \mathrm{He}$ and ${ }^{3} \mathrm{H}$ nuclei) plays a unique role in nuclear structure studies in general, and electron-scattering reactions in particular [72. This system is complex enough to include some fundamental nuclear

\footnotetext{
${ }^{2}$ It is worth stressing that spectral functions are not physical observables, and their extraction is entirely based on the PWIA assumption.
} 
environment effects (e.g. $N N$ interaction coming from different types of nucleonnucleon pairs, in contrast with the lighter deuteron system in which the interaction is only between a proton and a neutron), but its ground state can still be calculated very precisely (in contrast with heavier nuclei for which calculations are based on

effective models). Consequently, measurements of ${ }^{3} \mathrm{He}$ and ${ }^{3} \mathrm{H}\left(e, e^{\prime} p\right)$ cross sections can be used as a test of precise nuclear-theory calculations that are not feasible for heavier nuclei, and also as a test of calculations based on equation 2.5 .

\section{Isospin symmetry and $A=3$ doublet}

${ }^{3} \mathrm{He}$ and ${ }^{3} \mathrm{H}$ form an isospin doublet with total isospin quantum number $T=1 / 2$ and third component $T_{3}=(Z-N) / 2$ corresponding to $T_{3}^{3} \mathrm{He}=1 / 2$ and $T_{3}^{3} \mathrm{H}=-1 / 2$ respectively. That is, replacing all the protons with neutrons and all the neutrons with protons transforms either of these nuclei into the other:

$$
{ }^{3} \mathrm{He} \stackrel{p, n \leftrightarrow n, p}{\longleftrightarrow}{ }^{3} \mathrm{H} .
$$

Since the nuclear force is nearly isospin symmetric, protons and neutrons in ${ }^{3} \mathrm{He}$ should behave similarly to neutrons and protons in ${ }^{3} \mathrm{H}$ respectively. This can be tested by analyzing ratios of ${ }^{3} \mathrm{He}$ neutron to ${ }^{3} \mathrm{H}$ proton momentum distributions, which are more common than spectral function calculations (see Fig. 2-4). The isospin symmetry argument is valid within $3 \%$ in the nucleon momentum range of interest.

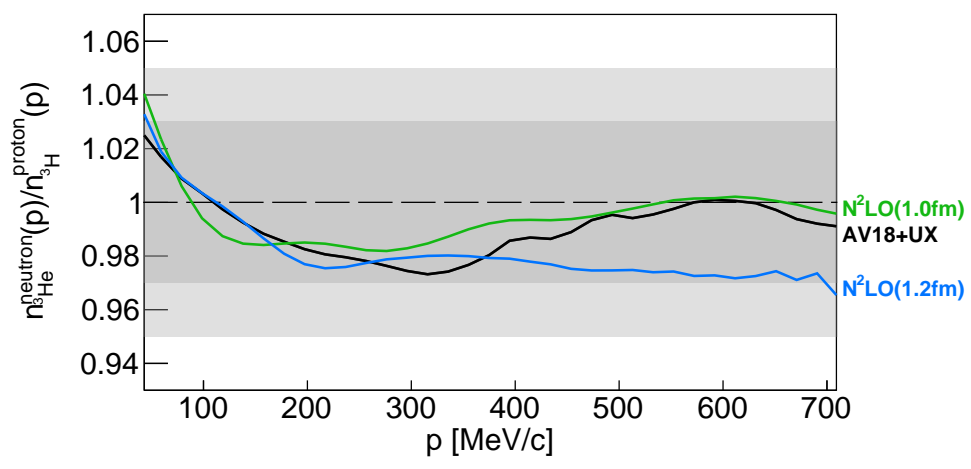

Figure 2-4: Ratio of ${ }^{3} \mathrm{He}$ neutron to ${ }^{3} \mathrm{H}$ proton momentum distributions as a function of nucleon momentum calculated using three different $N N$ models [66, 73]. The darkand light-gray areas correspond to the $\pm 3 \%$ and $\pm 5 \%$ bands respectively. 


\section{Bound (2bbu) and Continuum (3bbu) Final States}

There are two possible final states in the QE $\left(e, e^{\prime} p\right)$ scattering off ${ }^{3} \mathrm{He}$ :

- In the case that $E_{i}>E_{3_{\mathrm{He}, 3 b b u}^{\mathrm{bind}}} \equiv 2 m_{p}+m_{n}-m_{3 \mathrm{He}} \approx 7.7 \mathrm{MeV}$, then the scattering process breaks up the nucleus into three pieces: two protons and a neutron. Here, $m_{p}, m_{n}$, and $m_{3 \mathrm{He}}$ are the proton, neutron, and ${ }^{3} \mathrm{He}$ masses respectively. Therefore, the final state corresponds in this case to three-body kinematics, and the infinite ways in which energy and momentum can be shared between the final-state nucleons creates a continuous energy spectrum. This channel is known as the 3-body breakup (3bbu), or continuum channel, and labeled ${ }^{3} \mathrm{He}\left(e, e^{\prime} p\right) p n$.

- If $E_{i}=E_{3 \mathrm{He}, 2 b b u}^{\mathrm{bind}} \equiv m_{p}+m_{d}-m_{3 \mathrm{He}} \approx 5.5 \mathrm{MeV}$, then the scattering process breaks up the ${ }^{3} \mathrm{He}$ nucleus into two pieces: a proton and a deuteron. Here, $m_{d}$ is the deuteron mass. Therefore, the final state will correspond to 2-body kinematics, and the spectral function will depend on the separation energy as $\sim \delta\left(E_{i}-E_{3 \mathrm{He}, 2 b b u}^{\mathrm{bind}}\right)$. This channel is known as the 2-body breakup (2bbu), or bound channel, and labeled ${ }^{3} \operatorname{He}\left(e, e^{\prime} p\right) d$.

In the case of $\left(e, e^{\prime} p\right)$ scattering off ${ }^{3} \mathrm{H}$, the two neutrons left after the electronproton interaction do not form a bound state, and consequently only the 3bbu (or continuum) channel exists. The threshold for this reaction corresponds to $E_{i}>$ $E_{3_{\mathrm{H}}}^{\mathrm{bind}} \equiv m_{p}+2 m_{n}-m_{3_{\mathrm{H}}} \approx 8.5 \mathrm{MeV}$, and the energy spectrum is continuous, like that of the ${ }^{3} \mathrm{He} 3 \mathrm{bbu}$ channel. Here, $m_{3^{3} \mathrm{H}}$ is the tritium mass.

Fig. 2-5 shows the ${ }^{3} \mathrm{He}$ proton and neutron spectral functions calculated by $\mathrm{C}$. Ciofi degli Atti and L. P. Kaptari using the AV18 potential [69] as a function of nucleon momentum and energy. From isospin symmetry arguments, the ${ }^{3}$ He neutron spectral function can be interpreted as the ${ }^{3} \mathrm{H}$ proton spectral function. As expected, only the $3 \mathrm{bbu}$ channel is present in this case. 

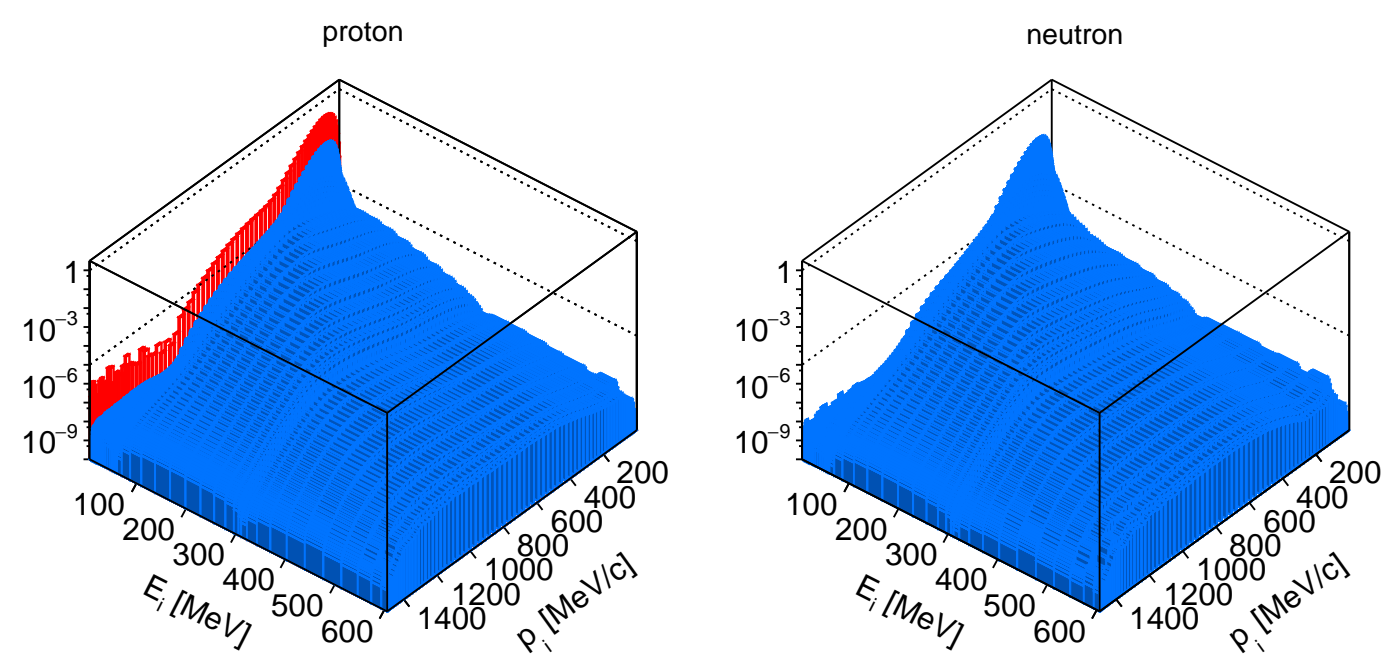

Figure 2-5: $A=3$ AV18 spectral function calculated by C. Ciofi degli Atti and L. P. Kaptari [69]. The left panel shows the ${ }^{3}$ He proton spectral function normalized to 2 (number of protons). The z-axis corresponds to $p_{i}^{2} S\left(p_{i}, E_{i}\right)$. The right panel shows the ${ }^{3}$ He neutron spectral function normalized to 1 (number of neutrons) which, following isospin-symmetry arguments, approximately equals the ${ }^{3} \mathrm{H}$ proton spectral function. The blue surfaces show the continuum or $3 \mathrm{bbu}$ channel. The red ridge, only present on the left panel, shows the bound or $2 \mathrm{bbu}$ channel.

\subsubsection{Suppression of non-QE reaction mechanisms}

Besides the processes from the diagrams illustrated in Fig. 2-3, there are several reaction mechanisms that lead to the same final-state particles and thus can affect or obscure the conclusions extracted from the measurement. The most significant of these are Meson-Exchange Currents (MEC), Isobar Currents (IC), and Final-State Interactions (FSI) (see diagrams in Fig. 2-6] [74, 75]. Minimizing the contribution from these reaction mechanisms is crucial for interpreting experimental results in the context of PWIA. This has been experimentally studied before in the case of QE $\mathrm{d}\left(e, e^{\prime} p\right)$ studies 45$]$.

\section{MEC}

MEC refers to the process in which the virtual photon transfers its energy and momentum to the virtual meson being exchanged between two interacting nucleons. Relative to the PWIA contribution, the amplitude for MEC is expected to be suppressed by 


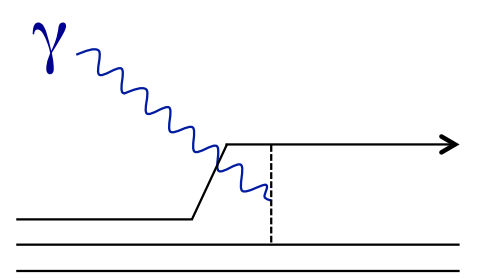

a) MEC

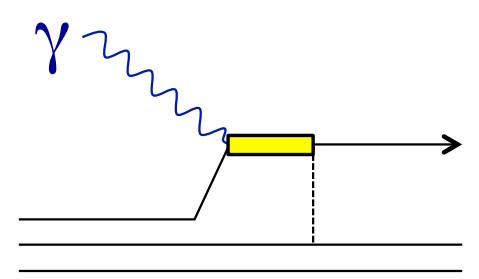

b) IC

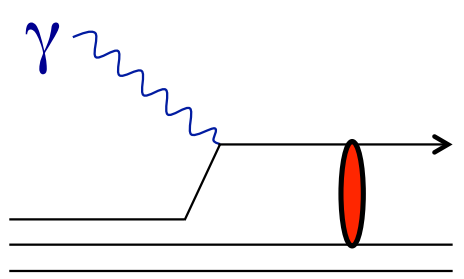

c) FSI

Figure 2-6: Non-QE reaction mechanisms with the same experimental signature as the diagrams in Fig. 2-3 a) Meson-Exchange Currents (MEC), b) Isobar Currents $(\mathrm{IC}), \mathrm{c})$ Final-State Interactions (FSI).

a factor of at least $\left(1+Q^{2} /\left(1\left[\left(\mathrm{GeV} / c^{2}\right)^{2}\right]\right)^{-2}[74\right.$, 75]. Therefore, this process can be reduced by going to high- $Q^{2}\left(Q^{2}>1.5\left(\mathrm{GeV} / c^{2}\right)^{2}\right)$ kinematics.

\section{IC}

IC refers to the process in which the virtual photon excites a nucleon into a $\Delta$ isobar configuration in the intermediate state, which subsequently re-scatters off another nucleon via $\Delta N \rightarrow N N$. Relative to the PWIA contribution, the amplitude for IC is expected to be suppressed by a factor of at least $1 / Q^{2}$. Therefore, this process can also be minimized by going to high- $Q^{2}$ kinematics. In addition, $\Delta$ is on-shell for $\omega=Q^{2} /\left(2 m_{p}\right)+300 \mathrm{MeV}$. Therefore, IC is reduced by going to kinematics at $\omega<Q^{2} /\left(2 m_{p}\right)$. Consequently requiring that $x_{B} \equiv Q^{2} /\left(2 m_{p} \omega\right)>1$ limits the selection to events far from the inelastic threshold, further suppressing the IC contribution.

\section{FSI}

FSI refers to the process in which the struck nucleon re-scatters from the other nucleons in the system. Its effect can be minimized by requiring that the angle between the momentum vector of the recoiling system and the $q$ vector $\left(\theta_{r q}\right.$, see Fig. 2-2 be small. To illustrate this, Fig. 2-7 shows the ratio of a ${ }^{3} \mathrm{He}\left(e, e^{\prime} p\right)$ cross section calculation which includes rescattering of the struck nucleon $\left(\sigma_{\text {Full }}\right)$, to the corresponding PWIA cross section calculation ( $\sigma_{\text {PWIA }}$ ) as a function of $\theta_{r q}$ by M. Sargsian. For very small or very large values of $\theta_{r q}, \sigma_{\text {Full }} / \sigma_{\text {PWIA }}$ approaches 1 . The maximum rescattering occurs for $\theta_{r q} \approx 70^{\circ}$. 


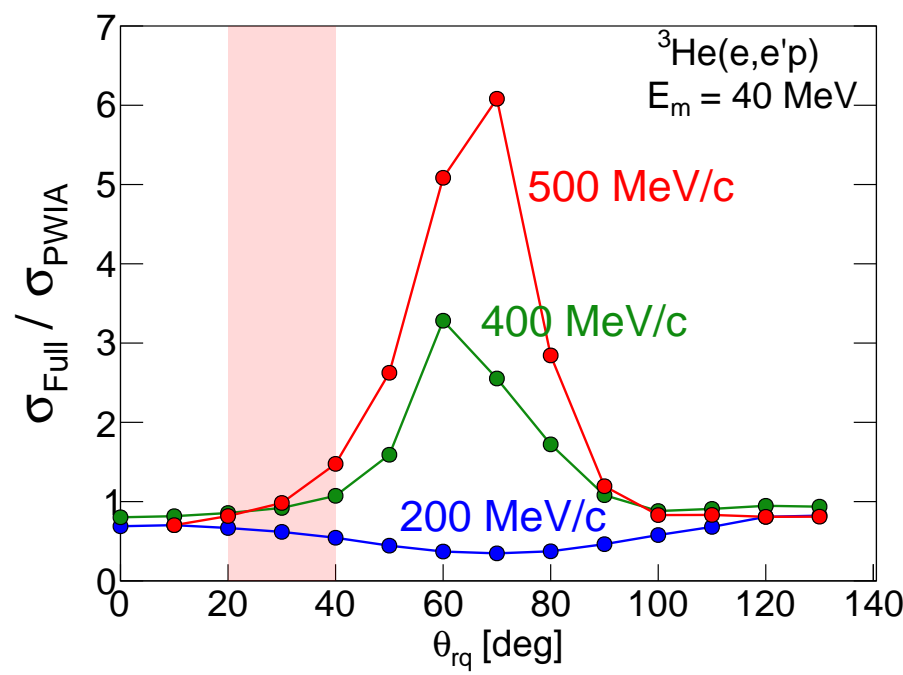

Figure 2-7: Ratio of calculated ${ }^{3} \mathrm{He}\left(e, e^{\prime} p\right)$ full cross section (which includes single rescattering of the struck nucleon off either one of the other two nucleons) to the PWIA cross section for $p_{\text {miss }}=200 \mathrm{MeV} / c$ (blue), $400 \mathrm{MeV} / c$ (green), and $500 \mathrm{MeV} / c$ (red) as a function of $\theta_{r q}$, the angle between the recoil momentum and $\vec{q}$ in the laboratory frame. The vertical band shows the acceptable upper limit for $\theta_{r q}$ to minimize FSI. Calculation by M. Sargsian.

Any residual FSI effect is expected to cancel in the cross section ratio of equal mass nuclei, since the knocked-out proton can rescatter off the same number of nucleons.

\subsection{3 $\left(e, e^{\prime} p\right) \quad$ cross-section calculations for few-body systems}

Theoretical calculations for the ${ }^{3} \mathrm{He}\left(e, e^{\prime} p\right)$ and ${ }^{3} \mathrm{H}\left(e, e^{\prime} p\right)$ cross sections are carried out using various techniques, and include contributions from different diagrams. For instance, in this work, we examine the Cracow-group calculations based on the Faddeev formulation of the three-body system [72, 76, 77] which are carried out either excluding or including $\mathrm{FSI}_{23}$ (see Fig. 2-3, b)), labeled Cracow-PW and Cracow respectively. Furthermore, we consider a calculation based on equation 2.5, using the ${ }^{3}$ He spectral function from C. Ciofi degli Atti and L. P. Kaptari (see Fig. 2-5) including $\mathrm{FSI}_{23}$ [69] and the $\sigma_{c c 1}$ electron-nucleon off-shell cross section [71], labeled $\mathrm{CK}+C C 1$. Finally, we also consider a calculation by M. Sargsian [78] that either excludes or includes FSI of the struck nucleon (see Fig. 2-6, c)) using the generalized Eikonal approximation [79, 80] (but excluding FSI $_{23}$ ), labeled Sargsian-PW and 
Sargsian-FSI respectively. The cross-section calculations available at the time of this study are listed in Table 2.1. We will test the effects of the different approximations against experimental data.

Table 2.1: List of available ${ }^{3} \mathrm{He}$ and ${ }^{3} \mathrm{H}\left(e, e^{\prime} p\right)$ cross-section calculations.

\begin{tabular}{c|ccc} 
& PWIA & PWIA + FSI $_{23}$ & PWIA +FSI \\
\hline \hline Cracow & $\checkmark$ & $\checkmark$ & \\
CK $+C C 1$ & & $\checkmark$ & \\
Sargsian & $\checkmark$ & & $\checkmark$
\end{tabular}

\subsubsection{Previous $A=3$ electron-scattering measurements}

There are many electron-scattering measurements on ${ }^{3} \mathrm{He}[81,82$, 83, 84, 85, 86, 87]. The most relevant to this thesis are the $\mathrm{QE}{ }^{3} \mathrm{He}\left(e, e^{\prime} p\right)$ 2- and 3-body breakup crosssection measurements from [42, 44]. These measurements were carried out at $Q^{2}=$ $1.5\left(\mathrm{GeV} / c^{2}\right)^{2}$ and $x_{B}=1$, near the expected maximum of struck-proton rescattering and, therefore, suffered from the reaction mechanisms described in section 2.2 .2 . The measured cross-sections were lower than PWIA calculations by a factor of $\sim 2$ for $p_{\text {miss }}<250 \mathrm{MeV} / c$, higher by a factor of $\sim 3$ for $400<p_{\text {miss }}<500 \mathrm{MeV} / c$, and even higher for larger $p_{m i s s}$, and were only accurately described by calculations that included large contributions from non-QE mechanisms [33, 43, 88, 89]. See Figs. 1-5 and 2-8. These calculations included both single- and double-rescattering, as well as meson exchange $(\pi$ and $\rho$ ) and IC. The interpretation of these results was limited due to the model dependence with which such calculations are carried out.

Tritium measurements, on the other hand, are much more infrequent due to safety limitations and regulations associated with this radioactive nucleus. ${ }^{3} \mathrm{He}$ and ${ }^{3} \mathrm{H}$, $\left(e, e^{\prime}\right)$ and $\left(e, e^{\prime} p\right)$ were measured in the early 1960's at the Stanford Linear Accelerator Center (SLAC) [90, 91, 92, 93]. In the late 1980's, additional $\left(e, e^{\prime}\right)$ measurements were carried out at MIT-Bates and Saclay [94, 95, 96, 97, 98, 99]. No new ${ }^{3} \mathrm{H}$ electron scattering data have been published since.

While the ${ }^{3} \mathrm{He}$ measurements are extremely important, the simultaneous measurement of the ${ }^{3} \mathrm{He}\left(e, e^{\prime} p\right)$ and ${ }^{3} \mathrm{H}\left(e, e^{\prime} p\right)$ reactions allows us to rigorously study the 
Figure 2-8: $\quad{ }^{3} \mathrm{He}\left(e, e^{\prime} p\right) p n \quad$ crosssection as a function of missing energy from 42. Each panel corresponds to a different bin in $p_{\text {miss. }}$. The black ( ) markers correspond to the measured cross section. The black dotted (....) curve presents a PWIA calculation using the spectral function from [100] and the $C C 1$ electron-proton cross section. The red dash-dotted (- - ), blue long-dashed (- - ), and green solid lines correspond respectively to PWIA, PWIA + FSI, and PWIA +FSI + MEC calculations by 43]. The pink thick-dashed $(-\mathbf{-})$ line, only present in the $p_{\text {miss }}=650$ $\mathrm{MeV} / c$ panel, corresponds to a calculation in which double rescattering has been turned off. The vertical arrow indicates the missing energy corresponding to scattering off a nucleon from a standing SRC pair. See text for details.

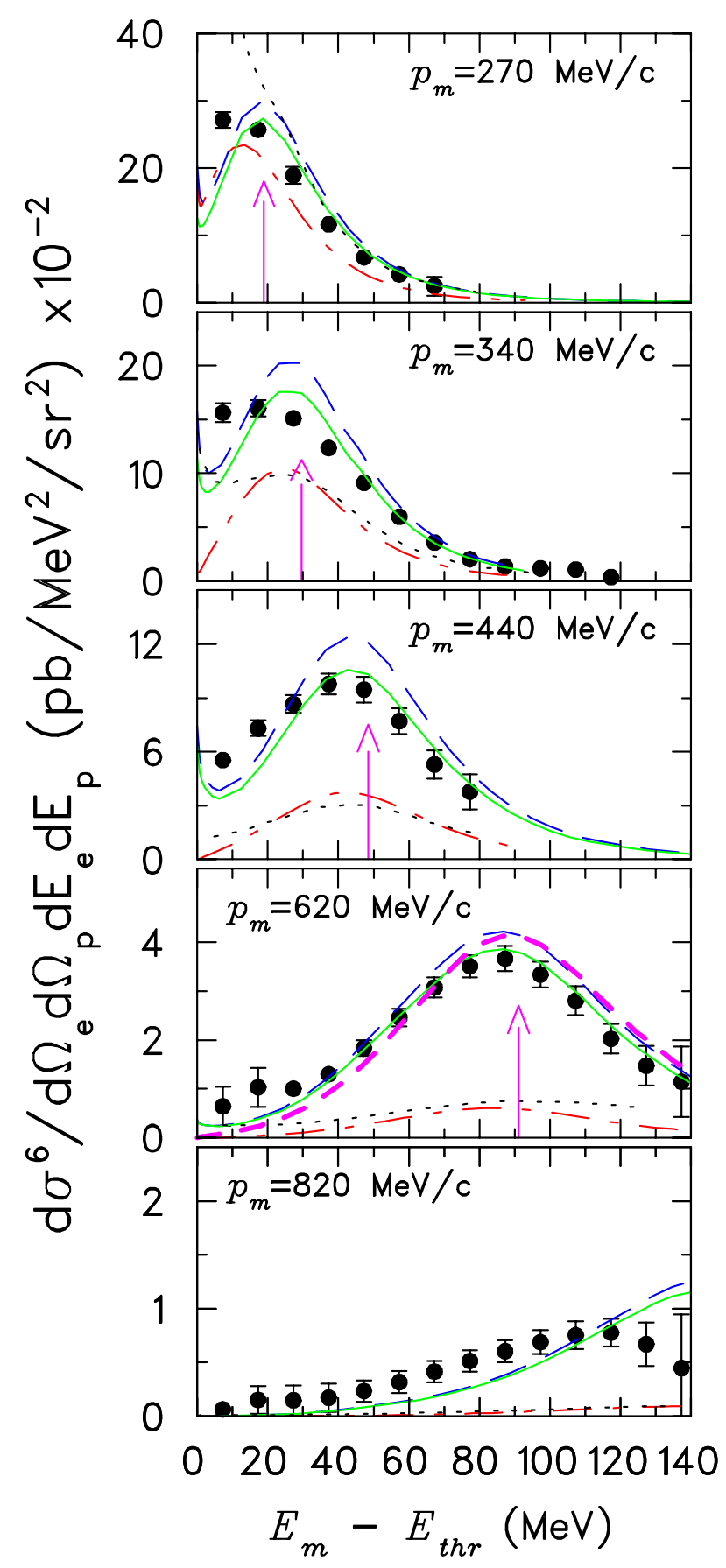


$A=3$ system as a whole, and further constrain the non-QE reaction mechanism and ground-state wave function models. Additionally, results from comparing the high$p_{\text {miss }}$ measured cross sections with calculations carried out using different models of the $N N$ interaction can be used to constrain $N N$ models at short distances. 


\section{Chapter 3}

\section{Measurement of $\left(e, e^{\prime} p\right)$ Scattering off ${ }^{3} \mathrm{H}$ And ${ }^{3} \mathrm{He}$}

[Phys. Lett. B 797, 134890 (2019), arXiv: 2001.07230 (2020)]

In this chapter, I will describe the first measurement of the ${ }^{3} \mathrm{He}$ and ${ }^{3} \mathrm{H}\left(e, e^{\prime} p\right)$ reactions in kinematics in which non-QE reaction mechanisms are expected to be minimal, and in the range $40 \leq p_{\text {miss }} \leq 500 \mathrm{MeV} / c$. The measurement took place in May 2018 in Hall A of the Thomas Jefferson National Accelerator Facility (JLab).

This chapter is organized as follows. Section 3.1 describes the experimental setup used in the measurement, which corresponded to the standard Hall-A equipment with a newly-designed target cell. Section 3.2 explains how the Hall-A $\left(e, e^{\prime} p\right)$ Monte Carlo event generator was modified to correctly simulate the $A=3$ cross section, and subsequently used to determine the kinematical settings for the experiment. Section 3.3 describes the detector calibration tasks in which I was most involved. Section 3.4 outlines the method used to corroborate that the experimental luminosity was well constrained. Section 3.5 reports in detail the data-analysis procedures carried out to extract the ${ }^{3} \mathrm{He} /{ }^{3} \mathrm{H}\left(e, e^{\prime} p\right)$ cross-section ratio [101] and ${ }^{3} \mathrm{He}$ and ${ }^{3} \mathrm{H}\left(e, e^{\prime} p\right)$ absolute cross sections [102. Lastly, sections 3.6 and 3.7 present and discuss our findings. 


\subsection{Experimental setup}

\subsubsection{JLab accelerator}

This experiment was carried out at JLab, which operates the Continuous Electron Beam Accelerator Facility (CEBAF) [103]. Electrons produced in an electron gun (photocathode) travel through the race-track-like loop shown in Fig. 3-1, and gain energy when traversing the north and south linear accelerators (linacs) located in the straight sections of the path. The electron beam can circle this race-track-like loop up to five times (gaining approximately $2.2 \mathrm{GeV}$ in every "pass") before being delivered to one of four experimental halls (labeled A through D).
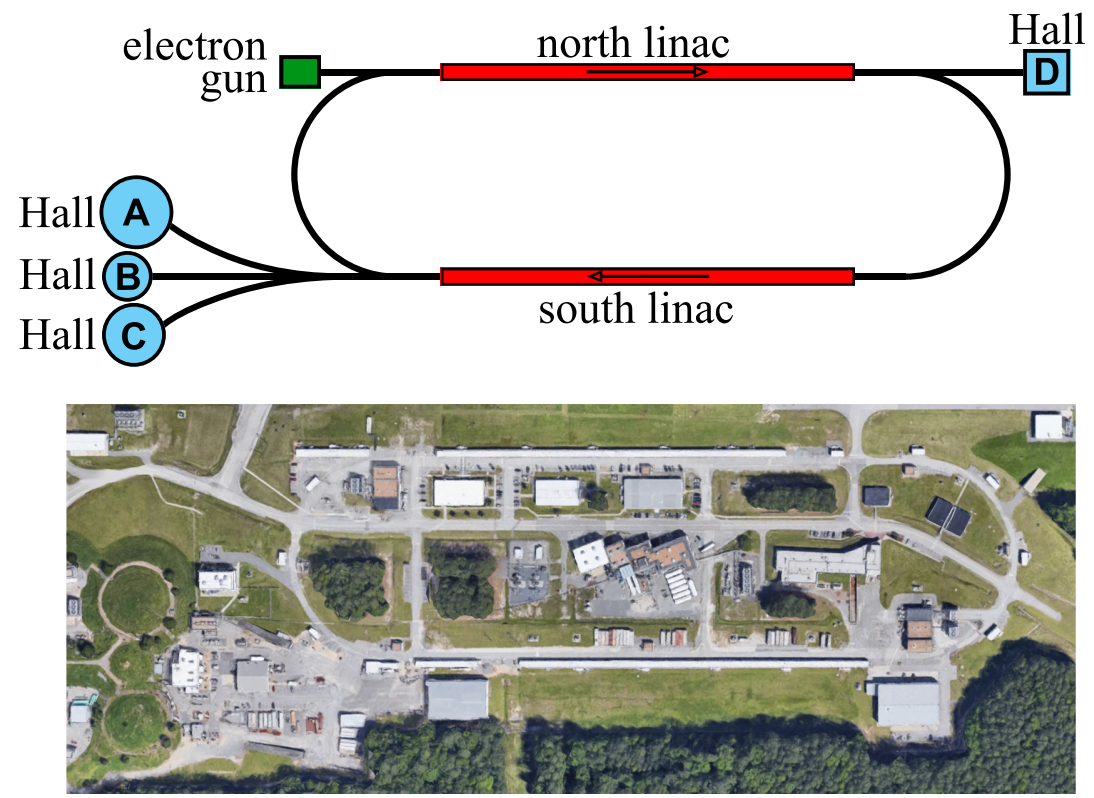

Figure 3-1: Aerial view of the Continuous Electron Beam Accelerator Facility (CEBAF) at JLab. Top: diagram showing the main features of CEBAF. The thick black lines describe the paths travelled by electrons as they are accelerated and delivered to the halls. Bottom: an aerial photo of CEBAF. All the components described in the diagram are underground. However, some service buildings, roads, and the hall roofs are visible from above.

In this experiment, a $2^{\text {nd }}$ pass beam was delivered to Hall $\mathrm{A}$, in which it impinged on a gaseous target located in the center of the hall. In the next sections, I will describe the experimental setup used to measure different quantities related to the incoming electron (3.1.2), nuclear target (3.1.3), and scattered electron and knocked- 
out proton (3.1.4). More details on all the information described in the rest of this section can be found in [104] and references therein.

\subsubsection{Hall-A beam line}

In this section, I will describe how the incoming-electron vector (beam direction and energy), as well as the total amount of electrons (delivered beam charge) are determined.

\section{Beam position and direction (Beam-Position Monitors and Raster)}

A combination of the transverse electron orbit in the accelerator (betatron motion) and other effects cause the transverse beam position at the target to be different from the nominal $(x, y)=(0,0)$ position. The transverse beam position is measured by a set of two Beam-Position Monitors (BPMs) located $7.524 \mathrm{~m}$ and $1.286 \mathrm{~m}$ upstream of the target respectively. Each BPM consists of four antennas oriented in the direction of the nominal beam path. As the beam travels through the BPM, each antenna measures a voltage. Comparing these signals allows the BPM system to determine the transverse beam coordinates in the BPM plane to within $100 \mu \mathrm{m}$ (for currents above $1 \mu \mathrm{A}$ ). Comparing the transverse beam coordinates determined by the two BPMs allows one to determine the incoming electron direction.

The BPM measurements are non-disruptive to the beam. However, before the BPMs can be used, they need to be calibrated against wire scanners called harps. The harp scans are beam disruptive measurements that consist of moving wires in different orientations across the beam path (at low currents) and reading out the induced wire signals. Two harps are positioned in the beam line (each adjacent to a BPM) and are surveyed with respect to the Hall A coordinate system by the JLab alignment group.

The heat transferred from the electron beam to the target can permanently damage the target cells. In order to avoid this problem, the heat load is spread out by rastering the beam. This is achieved using a set of four (two vertically- and two 
horizontally-deflecting) dipole magnets located $23 \mathrm{~m}$ upstream of the target. These dipoles deflect the beam in the vertical and horizontal directions at $\approx 25 \mathrm{kHz}$. A slight mismatch between the vertical and horizontal frequencies creates a Lissajouslike pattern. The raster is typically calibrated against the BPMs and is set to deflect the beam over a $2 \mathrm{~mm} \times 2 \mathrm{~mm}$ area. The raster induces a phase lag between the real beam position and the position recorded by the BPMs, and the BPMs only provide the average beam position. To get the event-by-event transverse beam position, the raster current is used instead.

\section{Beam energy}

This experiment was conducted with a $2^{\text {nd }}$ pass beam, which means a fixed nominal energy of approximately $4.4 \mathrm{GeV}$. We accurately measured the beam energy using the arc method.

The electron beam to Hall $\mathrm{A}$ is deflected by a series of 8 dipole magnets (see Fig. 3-2). The momentum of the electron beam is related to the bend angle of the arc and the field integral of the eight dipole magnets as:

$$
\left|\overrightarrow{p_{e}}\right|=k \frac{\int \vec{B} \cdot \overrightarrow{d l}}{\theta_{\text {bend }}},
$$

where $k=0.299792 \mathrm{GeV} \cdot \mathrm{rad} \cdot \mathrm{T}^{-1} \cdot \mathrm{m}^{-1} / c$. The bend angle can be measured using four harp scanners (two placed before, and two placed after the 8 dipoles). Since the eight dipoles are inaccessible, a 9th dipole, which is identical to and powered in series with the other eight, is used to measure the field integral. The absolute field measurement is done using a Nuclear Magnetic Resonance (NMR) probe, and the integral is determined via the "translating coil" method, consisting of moving two coils separated by a fixed distance through the dipole, and measuring flux changes in the two coils. Further details on how this procedure is carried out can be found in [105]. The measured beam energy was determined to be narrowly distributed around $E_{e}=4.3256 \mathrm{GeV}$. 


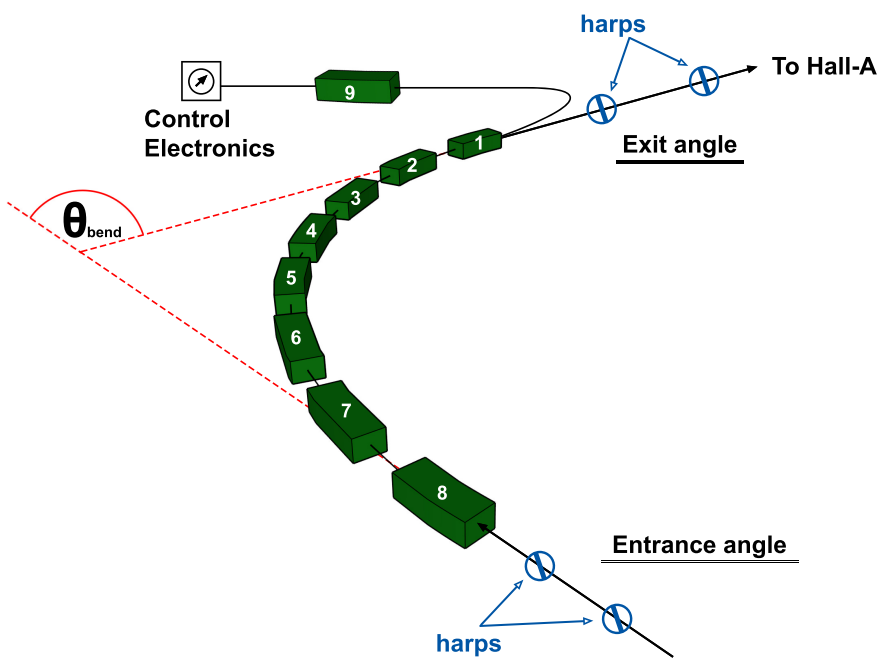

Figure 3-2: Absolute beam-energy measurement using the arc method.

\section{Beam current and charge (Beam-Current Monitor)}

The experiment was carried out at a nominal electron-beam current of $22.5 \mu \mathrm{A}$. The delivered beam current is measured continuously and non-intrusively (with an accuracy of $\leq 0.5 \%$ in the range $1-180 \mu \mathrm{A}$ ) using the Beam-Current Monitor (BCM) system (see Fig. 3-3) which is located $25 \mathrm{~m}$ upstream of the target system [106]. It consists of a parametric current transformer (referred to as an Unser) sandwiched between two Radio-Frequency (RF) cavity monitors enclosed in a temperature-stabilizing, magnetic-field-shielding box. The RF cavity monitors are stainless steel pill-box cavities tuned to the electron beam frequency $(1.497 \mathrm{GHz})$. An electron beam passing through the Unser and RF cavities induces a measurable frequency $(f)$ which is proportional to the input current (i.e. $I=($ gain $) \cdot f+$ offset). Measuring this frequency allows one to determine the beam current.

Two of the main advantages of the Unser are 1) it can be periodically self-calibrated and 2) its gain is very stable. Its main disadvantage is that its offset drifts significantly (at the $\mu \mathrm{A}$ level) on a time scale of several minutes and, consequently, cannot be used for a long-term current measurement. On the other hand, the RF cavities have the advantage that their signal is very stable (within $0.5 \%$ on the scale of several months), but the disadvantage that they cannot be self-calibrated. Thus, the BCM system is used by doing an Unser self-calibration, calibrating the RF cavities against the Unser 


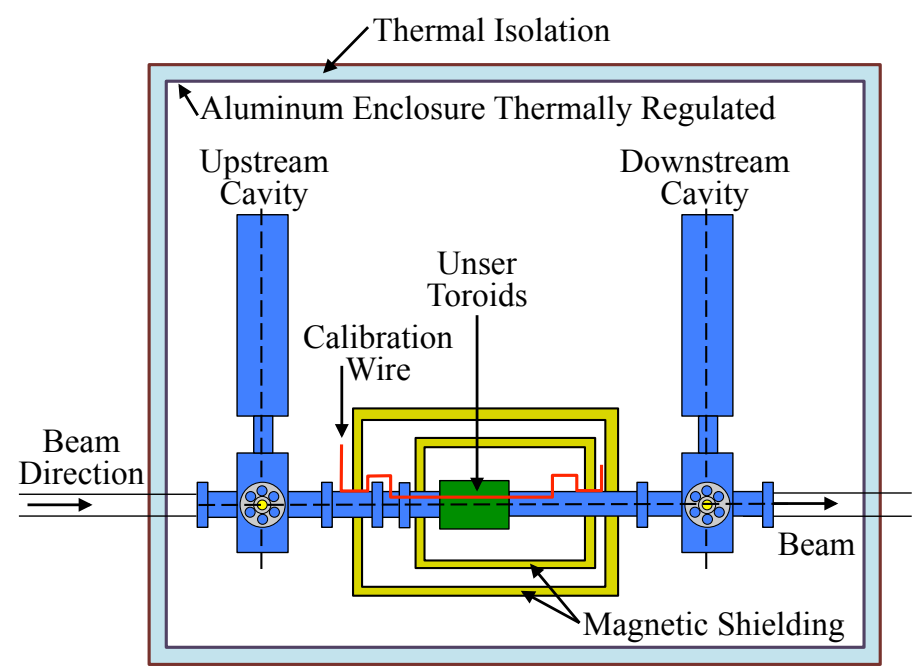

Figure 3-3: Schematic of the Hall-A Beam Current Monitor (BCM).

immediately after, and subsequently monitoring the current using the RF cavities. The calibration process for these devices is described in section 3.3.1.

The charge delivered by the incoming electron beam is determined by integrating the measured current over time.

\subsubsection{Tritium target assembly}

The Hall-A target system consists of a "ladder" that moves vertically to align a given target with the incoming electron beam (see Fig. 3-4). The ladder is equipped with five gas cells followed by a series of solid targets. The gas cells include tritium, deuterium, hydrogen, helium-3, and an empty cell for background studies. The solid targets are a 25-cm dummy and optics targets, and the carbon hole, raster, aluminum, carbon, titanium, and BeO targets.

\section{Gas cells}

The gas cells used in this experiment (see Fig. 3-5) correspond to sealed containers in which the gas is not circulated. This is different from the standard Hall-A highcurrent circulating-fluid target cells. The cell was designed to minimize the amount of tritium required and to provide several layers of tritium confinement. The cells were fabricated from aluminum 7075-T651. Additional considerations for the cell de- 

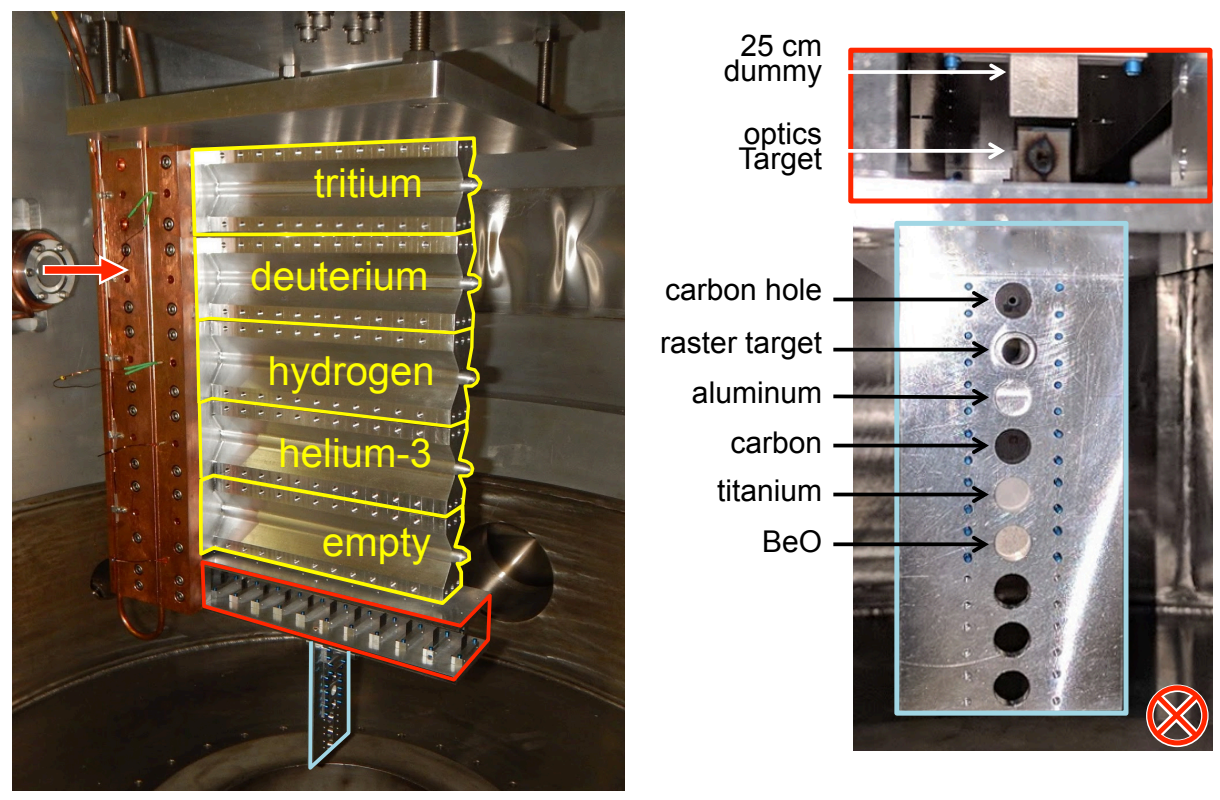

Figure 3-4: Target Assembly. (Left) Side view of target ladder used during the experiment. The areas marked in yellow show the gas target cells, which include (from top to bottom) tritium, deuterium, hydrogen, helium-3, and an empty cell for background studies. Below the gas target cells, we find the solid targets, which include the 25-cm dummy and optics targets (area marked in red) and the carbon hole, raster, aluminum, carbon, titanium, and $\mathrm{BeO}$ targets (area marked in light blue). (Right) Frontal view of the solid targets. The ladder moves up and down to position any selected target in front of the electron beam (the direction of which is represented by a red arrow (left) and cross (right)).

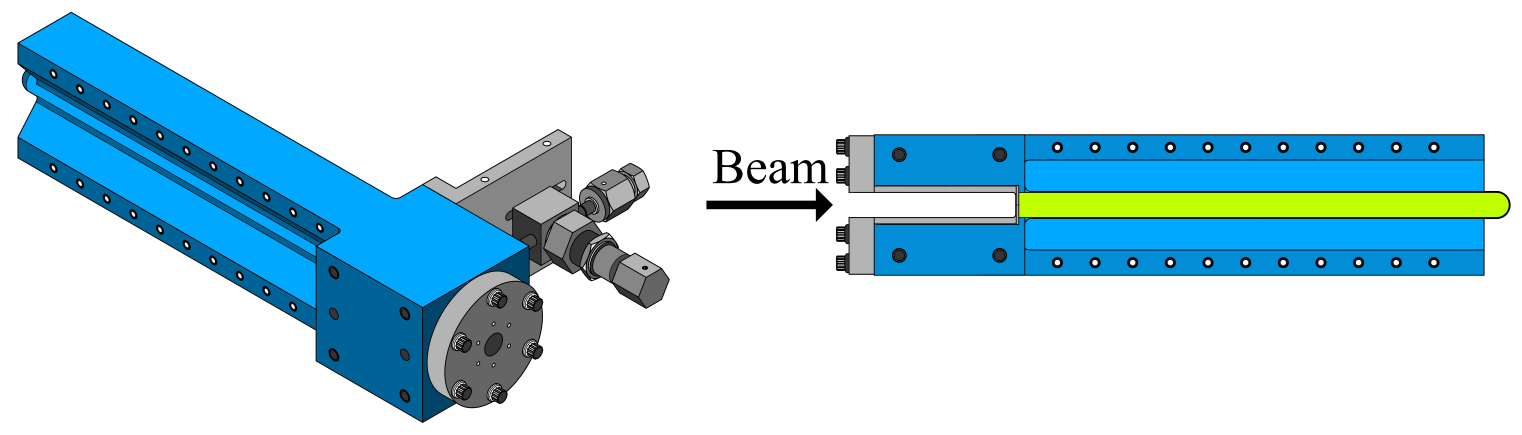

Figure 3-5: Schematic of the gas target cell. (Left) Cell design. (Right) Side view of the cell. The bright-green area represents the gas volume. 
sign included minimizing the aluminum volume to allow for the beam and scattered particles to enter and exit the cell with minimal effect from the target walls. Additional details can be found in [107, 108]. The gas densities and other properties are summarized in Table 3.1 .

Table 3.1: Properties of the gas targets used in the experiment. The thickness is obtained by multiplying the gas density $\left[\mathrm{mg} / \mathrm{cm}^{3}\right]$ by the target length $(25 \mathrm{~cm})$. See [107, 109, 110] for details.

\begin{tabular}{c|ccc} 
Target & Fill Pressure $[\mathrm{kPa}]$ & Fill Temp [K] & Thickness $\left[\mathrm{mg} / \mathrm{cm}^{2}\right]$ \\
\hline \hline${ }^{3} \mathrm{H}$ & 1400 & $296.3 \pm 0.1$ & $85.1 \pm 0.8$ \\
${ }^{2} \mathrm{H}(\mathrm{d})$ & 3549 & $296.1 \pm 0.1$ & $142.2 \pm 0.8$ \\
${ }^{1} \mathrm{H}$ & 3549 & $297.4 \pm 0.1$ & $70.8 \pm 0.4$ \\
${ }^{3} \mathrm{He}$ & 1772 & $294.3 \pm 0.1$ & $53.4 \pm 0.6$
\end{tabular}

\section{Solid targets}

In this experiment, the solid targets were only used for beam centering, raster, and optics calibrations. See section 3.3 for more details.

\subsubsection{High-Resolution Spectrometers (HRS)}

The scattered electron and knocked-out proton were measured using the standard Hall-A detector package, which consists of two (almost identical) High-Resolution Spectrometers (HRS) [104], labeled "Left" and "Right" according to the side of the beam line in which they are located, as shown on the left panel in Fig. 3-6. The target system is located at the center of the hall, and the spectrometers can independently pivot around this point to detect particles scattered at specific angles. In each HRS, four sequential magnets (three quadrupoles (Q), and a dipole (D), in a QQDQ sequence) focus and bend charged particles of a specific charge upwards to the detector package, as shown on the right panel in Fig. 3-6. By tuning the magnetic field in these magnets, one can study particles with momenta centered around a specific central momentum. The maximum momentum is about $4 \mathrm{GeV} / c$. 

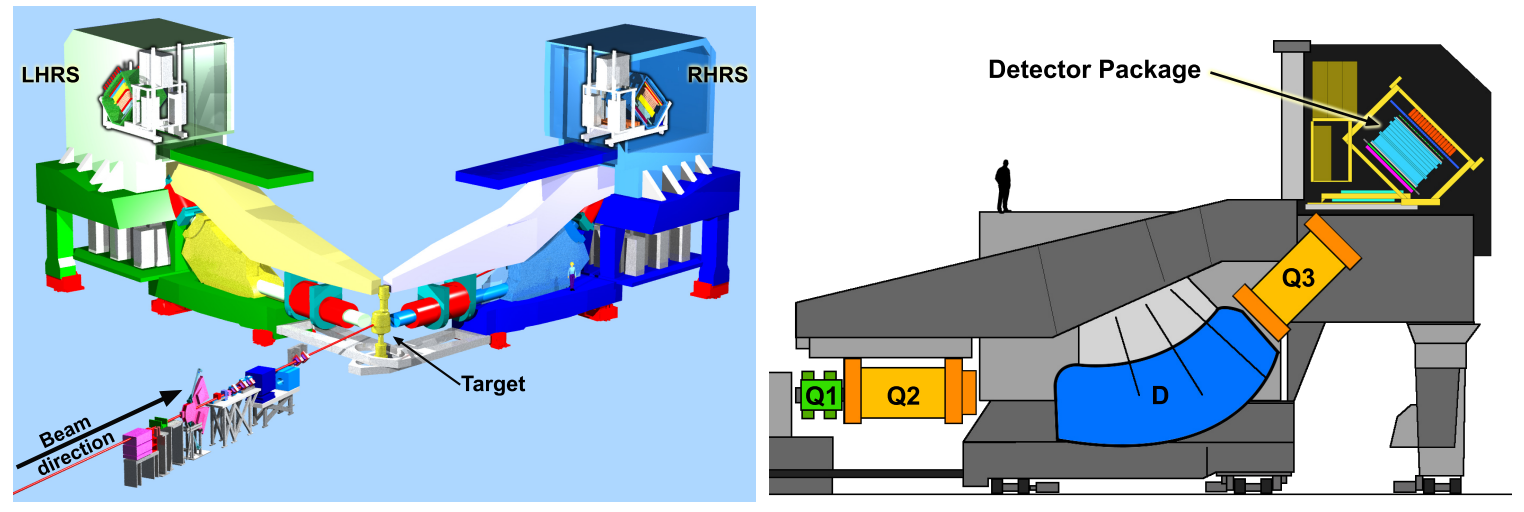

Figure 3-6: (Left) Hall-A experimental setup illustrating the beam-line components, target, and Left and Right High-Resolution Spectrometers (HRS). (Right) Side view of the HRS showing the QQDQ structure (where Q stands for quadrupole, and D stands for dipole), and the detector package.

\section{HRS acceptance}

The HRS momentum acceptance corresponds to $\delta \approx \pm 4.5 \%$ of the central spectrometer momentum value $p_{c}: p=p_{c}(1+\delta)$. The horizontal angular acceptance is approximately $\pm 30 \mathrm{mrad}$ around the spectrometer's central angle, and the vertical acceptance corresponds to approximately $\pm 60 \mathrm{mrad}$ about the horizontal. The Hall-A HRS acceptance is summarized in Table 3.2 .

Table 3.2: Hall-A HRS nominal momentum and angular acceptance.

\begin{tabular}{c|c} 
& acceptance \\
\hline \hline Momentum & $\pm 4.5 \%$ \\
Angular horizontal & $\pm 30 \mathrm{mrad}$ \\
vertical & $\pm 60 \mathrm{mrad}$
\end{tabular}

\section{HRS detector package}

A schematic of the HRS detector package is shown in Fig. 3-7. The main components of the detector package are a pair of vertical drift chambers (VDCs), which provide tracking information, and a pair of hodoscopes (scintillator detectors, labeled S0 and S2) which provide triggering (activation of data-acquisition system, DAQ) and timing information. These components are identical in both spectrometers. The remaining components, a $\mathrm{CO}_{2}$ Cherenkov detector and a lead-glass calorimeter, provide particle 
identification.

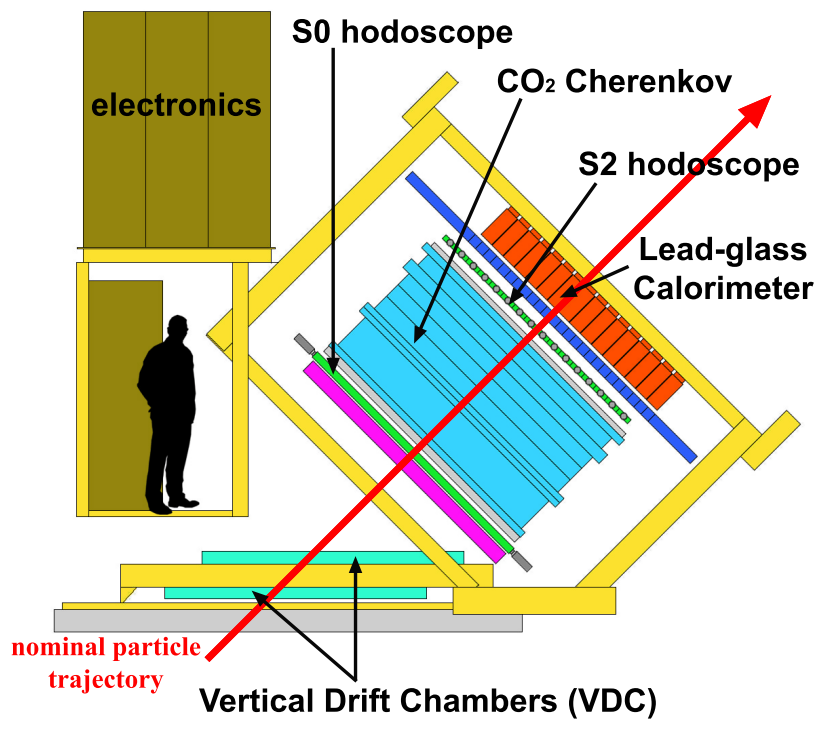

Figure 3-7: Schematic of the HRS detector package. Particles traverse the detector from left to right, and from below at $45^{\circ}$.

\section{Vertical Drift Chambers}

A pair of Vertical Drift Chambers (VDC), separated by $335 \mathrm{~mm}$, provide tracking information in each HRS [111] (see Fig. 3-7). Each VDC consists of two wire planes in a UV configuration, with the wires in the $\mathrm{U}$ and $\mathrm{V}$ planes in a $90^{\circ}$ angle with respect to one another, and the two planes together in a $45^{\circ}$ angle with respect to the nominal particle trajectory, as shown in Fig. 3-8. Each ( $\mathrm{U}$ and $\mathrm{V}$ ) plane is composed of 368 sense wires separated by $4.24 \mathrm{~mm}$. The active volume of each VDC consists of a gas mixture of argon $(62 \%)$ and ethane (38\%). The sense wires are at a potential of $3.5 \mathrm{kV}$.

When a charged particle crosses this gas mixture, it ionizes the gas molecules. The resulting electrons are accelerated by the electric field towards the closest sense wire, creating an electrical signal in that wire. The wire which the electron drifted toward, and the hit timing information are recorded. Information from four hits are used to reconstruct a track. In the spectrometer focal plane, defined by the lower VDC layer, the position resolution is $\sigma_{x, y} \sim 100 \mu \mathrm{m}$, and the angular resolution is 

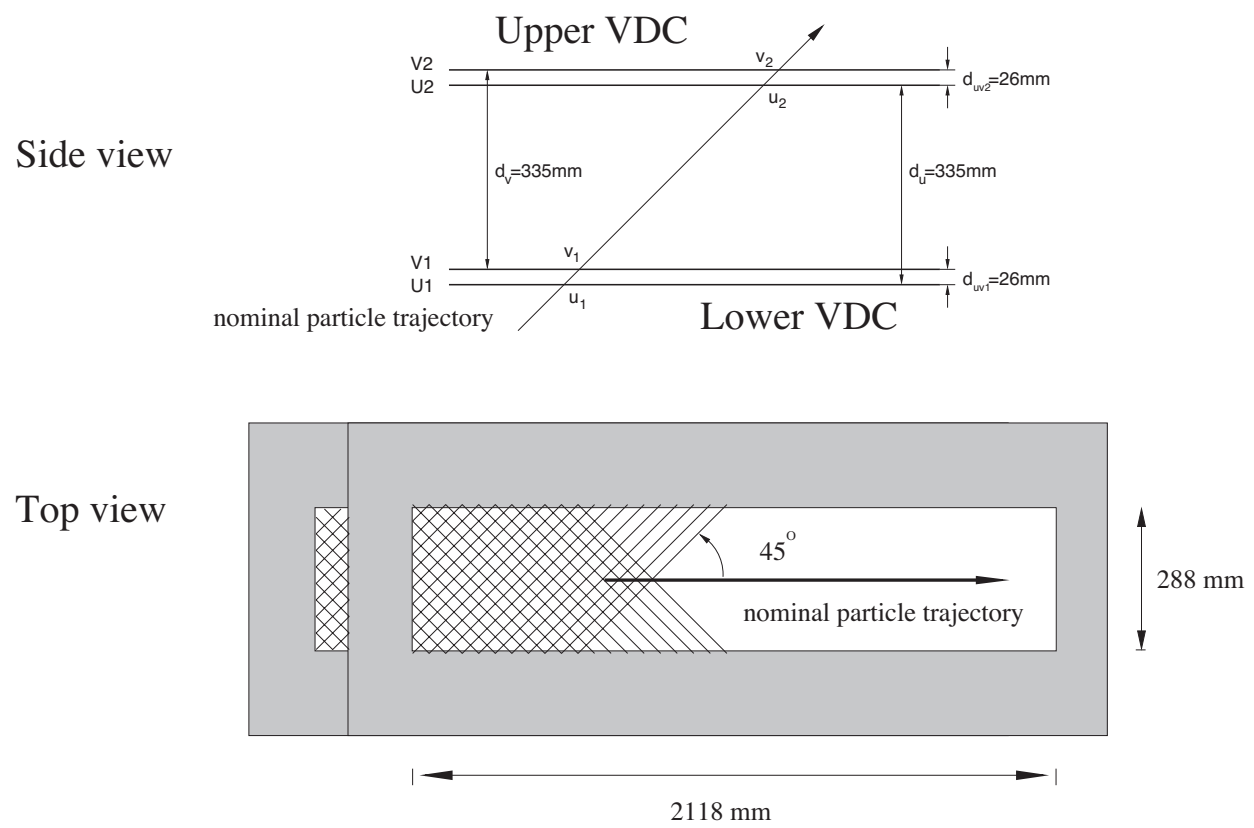

Figure 3-8: Schematic layout for the two Vertical Drift Chambers (VDC) in each Hall-A HRS. Figure taken from [104]. The top and bottom schematics show the side and top views of the VDC respectively. 
$\sigma_{\theta, \phi} \sim 0.5 \mathrm{mrad}$. This, combined with the long optical path $(\approx 23.4 \mathrm{~m}$ from target to focal plane) allows for a momentum resolution of about $2 \times 10^{-4}$ and an angular resolution of about $2 \mathrm{mrad}$ at the target.

\section{Hodoscopes}

In each HRS, a pair of hodoscope detectors labeled S0 and S2 provide triggering and timing information. The S0 detector consists of a long scintillator paddle read out from above and below by two Photo-Multiplier Tubes (PMT). Approximately $2 \mathrm{~m}$ after S0 in the particle path, the S2 hodoscope consists of 16 slightly overlapping paddles, each read out from both sides by two PMTs. See Fig. 3-9. The active areas of the S0 and S2 hodoscopes are approximately $190 \times 40 \mathrm{~cm}^{2}$ and $220 \times 54 \mathrm{~cm}^{2}$ respectively.

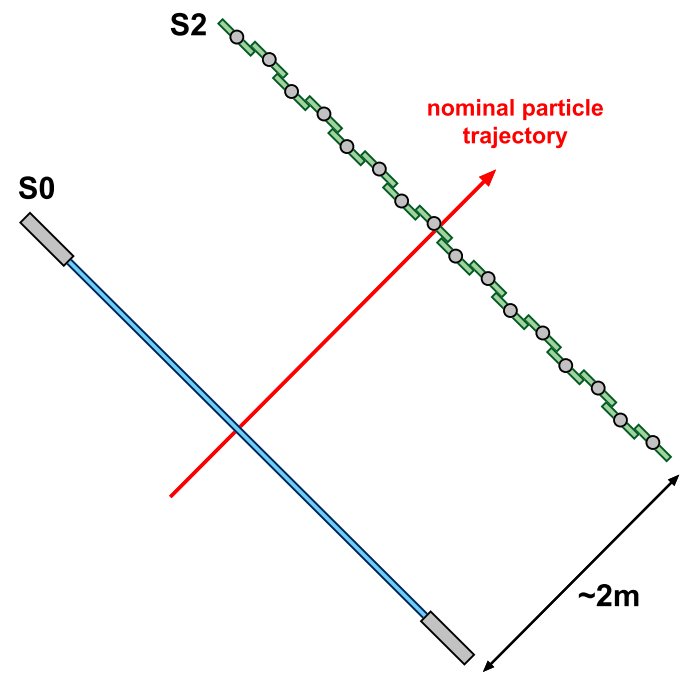

Figure 3-9: Schematic layout (side view) for the hodoscopes in each Hall-A HRS. The blue and green areas represent the S0 and S2 scintillators respectively. The gray areas indicate PMTs.

The Hall-A hodoscopes work through the principle of scintillation. When charged particles traverse a scintillator, they excite the scintillator molecules in their path. As these molecules de-excite, they emit light that, when coupled to a PMT, is converted into an electrical signal, amplified, and recorded. Compared to other detectors, scintillators have a very fast time response and, consequently, are commonly used for timing purposes. For more details about scintillation see, e.g., [112]. 
The long optical path from the interaction point to the HRS detector package, combined with the fast response of scintillator detectors, allows for time-of-flight particle identification (relative to detected electrons) with a resolution $\sigma \sim 0.5 \mathrm{~ns}$ in the case of coincidence experiments such as this on $\varrho^{1}$.

\section{Cherenkov detector}

$\mathrm{A} \mathrm{CO}_{2}$ Cherenkov detector (filled at atmospheric pressure, $n=1.00041$ ) is installed in each spectrometer between the S0 and S2 scintillator detectors [113]. The Cherenkov light is focused onto 10 PMTs by 10 spherical mirrors.

When a charged particle travels through a medium (of refraction index $n$ ) with velocity $v=\beta c$ greater than the speed of light in that medium, a characteristic cone of light with an opening angle $\theta_{\breve{C}}$ such that $\cos \theta_{\breve{C}}=(\beta n)^{-1}$ called Cherenkov radiation is emitted. Since the cosine function takes a maximum value of 1 , the threshold for this Cherenkov radiation to be emitted corresponds to $\beta=1 / n$, and consequently, the momentum threshold for a particle of mass $m$ is:

$$
p_{\check{C} \text { threshold }}=\frac{m c}{\sqrt{n^{2}-1}}
$$

Because of the mass dependence, we use Cherenkov detectors for particle identification. In the Hall-A Cherenkov detectors, the Cherenkov radiation threshold for pions is $p_{\pi}>4.8 \mathrm{GeV} / c$, and even higher for heavier particles. These detectors identify electrons with $99 \%$ efficiency. The Cherenkov detector signals can be combined with the hodoscope signals to create different trigger configurations.

\section{Lead-Glass calorimeter}

There are two calorimeter layers in each Hall-A HRS. Each layer is composed of several tens of $\left(\sim 10 \times 10 \times 30 \mathrm{~cm}^{3}\right)$ lead-glass blocks. The exact size of the blocks, their number, and orientation, is slightly different between each calorimeter layer and also between the two spectrometers [104].

\footnotetext{
${ }^{1}$ This limit is actually set by the readout electronics used in the experiment and not by the scintillators.
} 
When a high-energy electron (or positron) interacts in a dense medium, it loses energy by the emission of a photon (this process is called Bremsstrahlung, see section 3.6.3 for details). This photon then produces an electron-positron pair. This pair, and the original electron (or positron) go on to radiate more photons that consequently pair-produce more electrons and positrons. As this process is repeated many times, a cascade of particles (referred to as electromagnetic shower) is produced. This shower continues to develop until the average energy of the particles falls below a critical energy (which depends on the atomic number of the material).

When hadrons (both charged and neutral) interact strongly with the atomic nuclei in the same medium, they can produce particles that interact further downstream, producing a cascade of particles (referred to as hadronic shower). Electromagnetic and hadronic showers develop very differently. While the former is more uniform (repeating Bremsstrahlung and pair-production steps), the latter is more variable, since many more final states are produced in high-energy hadronic interactions. Additionally, neutral pions produced in the interaction decay via $\pi^{0} \rightarrow \gamma \gamma$, leading to an electromagnetic component in the shower. Moreover, about a third of the incident energy is lost in the form of nuclear excitation, making this energy undetectable. Furthermore, electromagnetic showers develop and die off in a smaller volume than hadronic ones. All these differences between the two types of showers make their detection (with calorimeters) a useful particle-identification method.

\subsubsection{Trigger setup and efficiency}

We recorded all events which had an electron-spectrometer trigger. The trigger setup for this experiment is summarized in Table 3.3. The DAQ was configured to record events for which any of the LHRS triggers (T1 through T3) fired. Additionally, the information from the remaining triggers was saved onto the data stream.

To determine the T1 efficiency, $\epsilon_{\mathrm{T} 1}$, we begin by selecting a good electron sample $N_{\text {tot }}$ with nominal acceptance cuts, a single track reconstructed by the VDCs, a good signal in the Cherenkov detector, and by requiring that the events fired T3. Of this sample, we determine $N_{\mathrm{T} 3}$, the number of events that fired T3, but neither T1 nor 
Table 3.3: Trigger setup. S0 and S2 refer to signals in the first and second scintillator planes encountered by particles traversing the detectors. GC refers to a signal in the gas Cherenkov counter. The symbols " $\&$ " and "||" represent the logical operators "and" and "or" respectively.

\begin{tabular}{c|cc} 
& Label & Trigger Logic \\
\hline \hline \multirow{3}{*}{ LHRS trigger } & T1 & $\left.($ S0 \& S2 $)\right|_{\text {LHRS }}$ \\
& T2 & $\left(\right.$ S0 \& S2 \& GC $\left.\right|_{\text {LHRS }}$ \\
& T3 & $\left(\right.$ S0 || S2) \& GC $\left.\right|_{\text {LHRS }}$ \\
\hline RHRS trigger & T4 & $\left.($ S0 \& S2 $)\right|_{\text {RHRS }}$ \\
\hline \multirow{3}{*}{ Coincidence } & C1 & T1 \& T4 \\
& C2 & T1 \& S2 $\left.\right|_{\text {RHRS }}$ \\
& C3 & T1 \& S0 $\left.\right|_{\text {RHRS }}$
\end{tabular}

T2. Consequently, $N_{\mathrm{T} 3} / N_{\text {tot }}$ corresponds to the combined S0 and S2 inefficiency (if T3 fired, but T1 did not, it is because either S0 or S2 did not fire). This is illustrated in the left Venn diagram from Fig. 3-10. T1 is represented by the entire red area (including the purple overlap with T3). T3 is represented by the entire blue area (including the purple overlap with T1). T2 is represented by the purple overlap between the red and blue areas. $N_{\mathrm{T} 3}$ corresponds to the gridded area, and $N_{\text {tot }}$ to the entire blue area (including the purple overlap with $\mathrm{T} 1$ ). Thus, the T1 efficiency is determined as:

$$
\epsilon_{\mathrm{T} 1}=1-\frac{N_{\mathrm{T} 3}}{N_{\mathrm{tot}}} \approx 99.7 \pm 0.1 \% .
$$
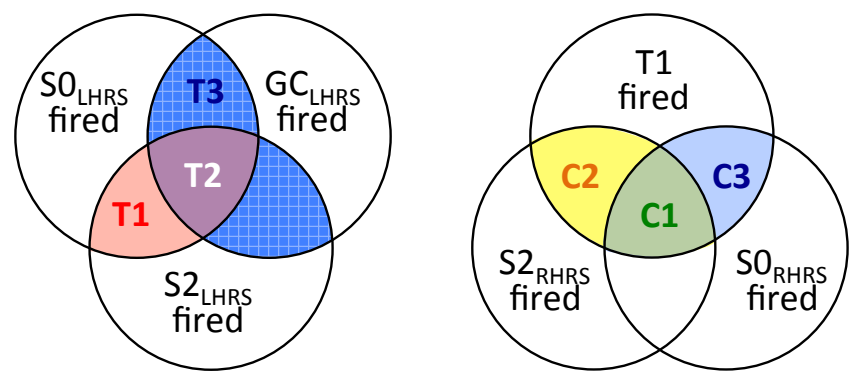

Figure 3-10: Venn diagram illustrating the relationship between different triggers: (Left) LHRS, (Right) Coincidence. Note that the red and blue areas on the left diagram include the purple area from the overlap. Similarly, the yellow and blue areas on the right diagram include the green area from the overlap. 
The T4 efficiency, $\epsilon_{\mathrm{T} 4}$, is determined by the RHRS S0 and S2 efficiencies:

$$
\epsilon_{\mathrm{T} 4}=\epsilon_{\left.\mathrm{S} 0\right|_{\mathrm{RHRS}}} \times \epsilon_{\left.\mathrm{S} 2\right|_{\mathrm{RHRS}}}=\left(\frac{N_{\mathrm{C} 1}}{N_{\mathrm{C} 2}}\right) \times\left(\frac{N_{\mathrm{C} 1}}{N_{\mathrm{C} 3}}\right) \approx 98.4 \pm 0.1 \%
$$

where $N_{\mathrm{C} 1}, N_{\mathrm{C} 2}$, and $N_{\mathrm{C} 3}$ are the number of events that fire triggers $\mathrm{C} 1, \mathrm{C} 2$, and C3 respectively, and in which the RHRS particle satisfies the same acceptance and single-track cuts required in the $\epsilon_{\mathrm{T} 1}$ calculation, and are represented by the green, yellow (including the green overlap), and blue (including the green overlap) areas in the right Venn diagram from Fig. 3-10 respectively.

Coincidence events were selected from the difference between the electron and proton event times (T1-T4). Therefore, the coincidence-trigger efficiency is defined as:

$$
\epsilon_{\mathrm{coinc}}=\epsilon_{\mathrm{T} 1} \times \epsilon_{\mathrm{T} 4} \approx 98.1 \pm 0.1 \%
$$

\subsection{Simulation studies}

SIMC is a Monte Carlo event generator that emulates the Hall-A experimental conditions [114]. SIMC generates events following the QE $\left(e, e^{\prime} p\right)$ PWIA cross-section model from equation 2.5. Radiation, Coulomb, energy loss, and other corrections can be added to the generated data. Subsequently, these events are propagated through a realistic HRS model to account for detector acceptance and resolution effects. These events can then be analyzed just as measured data.

\section{$A=3$ cross section}

The standard SIMC package could be used to simulate the $\mathrm{d}\left(e, e^{\prime} p\right)$ breakup and $A \geq 3$ continuum reactions. In the case of $\mathrm{d}\left(e, e^{\prime} p\right)$, the cross section is calculated following equation 2.5 with the spectral function:

$$
S_{d}\left(\left|\vec{p}_{i}\right|, E_{i}\right)=n_{d}\left(\left|\vec{p}_{i}\right|\right) \delta\left(E_{i}-E_{d}^{\text {bind }}\right)
$$


where $n_{d}$ is the deuteron 1-body momentum distribution from Wiringa [115], and $E_{d}^{\text {bind }}$ is the deuteron binding energy. In the case of $A \geq 3$ continuum reactions, the cross section is calculated using a series of spectral functions by Benhar et al. [116].

I added to SIMC the ${ }^{3} \mathrm{He}$ spectral function calculated by C. Ciofi degli Atti and L. P. Kaptari [69] and shown in Fig. 2-5. Due to the lack of a ${ }^{3} \mathrm{H}$ proton spectral function, SIMC was modified to assume isospin symmetry, and the ${ }^{3} \mathrm{H}\left(e, e^{\prime} p\right)$ calculation was carried out using the ${ }^{3} \mathrm{He}$ neutron spectral function from [69] instead. As shown in section 2.2.1. this assumption is valid within $\approx 3 \%$ in the $p_{\text {miss }}$ range studied.

Finally, the ${ }^{3} \mathrm{He}\left(e, e^{\prime} p\right)$ 2-body breakup (2bbu) channel needed to be added to the simulation. To achieve this, I created a new, independent simulation mode. In this new mode, SIMC treats the kinematics for scattering off ${ }^{3} \mathrm{He}\left(e, e^{\prime} p\right)$ identically to $\mathrm{d}\left(e, e^{\prime} p\right)$, with the replacement of the appropriate variables (e.g. target mass: $m_{d} \rightarrow$ $m_{3 \mathrm{He}}$, and binding energy: $\left.E_{d}^{\text {bind }} \rightarrow E_{3_{\mathrm{He}}, 2 b b u}^{\text {bind }}\right)$, and using the $2 \mathrm{bbu}$ spectral function from [69]. The 2- and 3-body breakup simulations are then run independently, and subsequently added.

\section{Kinematics selection and optimization}

We chose $Q^{2}=2.0\left(\mathrm{GeV} / c^{2}\right)^{2}$, and $x_{B}=1.4$ to reduce non-QE reaction mechanisms. We then chose a $2^{\text {nd }}$ pass beam $\left(E_{e}=4.4 \mathrm{GeV}\right)$ so the scattered electron could be detected in the HRS. This gave a central angle and momentum for the scattered electron in the LHRS of $\left(\theta_{e^{\prime}}, p_{e^{\prime}}\right)=\left(20.88^{\circ}, 3.543 \mathrm{GeV} / c\right)$.

We ran simulations for these conditions and considering knocked-out protons with any final-state momentum and angles. Given the limited HRS acceptance, two separate kinematical settings were needed to cover a missing momentum range of $40 \leq p_{\text {miss }} \leq 550 \mathrm{MeV} / c$, with the low- $p_{\text {miss }}$ kinematical setting extending up to about $p_{\text {miss }} \approx 250 \mathrm{MeV} / c$, and the high- $p_{\text {miss }}$ kinematical setting covering higher $p_{\text {miss }}$. This range ensured complete coverage of the transition between single-particle and twonucleon SRC behavior. Two (one for each kinematical setting) large-acceptance $\theta_{p}$ vs. $p_{p}$ histograms were created (with counts in the $z$ axis), only including events for which $\theta_{r q}<37.5^{\circ}$ to minimize FSI (see section 2.2.2). Since the cross section 
is larger at lower- $p_{\text {miss }}$, the low- $p_{\text {miss }}$ kinematical setting is determined by scanning the resulting $\theta_{p}$ vs. $p_{p}$ histogram with a "box" the size of the HRS angular (horizontal) - momentum acceptance (see Table 3.2) and determining the spectrometer central kinematics as the box position which yields the maximum number of counts. The candidate high- $p_{m i s s}$ kinematical setting was selected by additionally requiring events for which $p_{\text {miss }}>300 \mathrm{MeV} / c$, and then repeating the process of scanning this $\theta_{p}$ vs. $p_{p}$ histogram with the acceptance box. The resulting low- and high- $p_{\text {miss }}$ kinematical settings were determined to be $\left(\theta_{p}, p_{p}\right)=\left(48.82^{\circ}, 1.481 \mathrm{GeV} / c\right)$ and $\left(58.50^{\circ}, 1.246 \mathrm{GeV} / c\right)$ respectively.

An additional kinematical setting was determined similarly for the measurement of $\mathrm{H}\left(e, e^{\prime} p\right)$ elastic scattering. This kinematically-overconstrained reaction is used for calibration and resolution studies. In order to minimize RHRS motion, the protonarm kinematical setting was fixed to be that of the low- $p_{m i s s}$ kinematics, and the $\theta-p$ plot used for the kinematics determination was created with electron variables. The experimental kinematics are summarized in Table 3.4 .

Table 3.4: Kinematical settings used in the experiment.

\begin{tabular}{c|c|c} 
& LHRS setting $\left(\theta_{e^{\prime}}, p_{e^{\prime}}\right)$ & RHRS setting $\left(\theta_{p}, p_{p}\right)$ \\
\hline \hline high- $p_{\text {miss }}$ kinematics & \multirow{2}{*}{$20.88^{\circ}, 3.543 \mathrm{GeV} / c$} & $58.50^{\circ}, 1.246 \mathrm{GeV} / c$ \\
\cline { 1 - 1 } \cline { 1 - 1 } low- $p_{\text {miss }}$ kinematics & & $48.82^{\circ}, 1.481 \mathrm{GeV} / c$
\end{tabular}

We also measured $\mathrm{d}\left(e, e^{\prime} p\right)$ in the low- $p_{\text {miss }}$ kinematical setting.

\subsection{Calibrations}

The JLab Hall-A Tritium program included four experiments run by dozens of physicists, including over 13 graduate students. While the physics goals varied, the calibration measurements were done concurrently, and shared among experiments. Here, I summarize the calibration tasks in which I was most involved. 


\subsubsection{BCM calibration}

The BCM system is calibrated in two steps: 1) the Unser is self calibrated (without electron beam), and 2) the RF cavity monitors are then calibrated against the Unser (with electron beam).

The Unser is calibrated by passing a known DC current through an internal wire, and creating a map between the frequency measured by the Unser and the input current. The current is passed through the wire in 90-second steps, increasing the wire current in every step $(2,3,5,10,25,40,60,80,90,100 \mu \mathrm{A})$ (see Fig. 3-11 a)). The wire current is turned off before and after each step. These zero-current steps are measured to subtract the Unser DC background (pedestal). This sequence can be repeated to improve the measurement statistics. After the pedestal has been subtracted, the frequency distribution measured in each current step is fitted with a normal distribution, and the resulting mean is taken to be the measured frequency. The error in the Unser frequency response is given by $\frac{1.5 \mu \mathrm{A}}{\sqrt{\text { step size }}}($ where step size $=90 \mathrm{sec})$. I plotted input DC current vs. Unser frequency and fitted it with a linear function $I_{\text {input }}=\left(\right.$ gain $\left._{\text {Unser }}\right) \cdot f_{\text {Unser }}+$ offset $_{\text {Unser }}$. The fit parameters correspond to the Unser gain (stable) and offset (drifting). See Fig. 3-11 b).

The RF cavity calibration requires turning the electron beam on and off several times, and simultaneously measuring the RF cavity and Unser responses. A beam profile corresponding to 90 -second steps of $3,7,12,18,22.2 \mu \mathrm{A}$ with additional $0 \mu \mathrm{A}$ steps between each step is requested. Since the calibration is done with the beamline equipment, the nuclear target in the hall is irrelevant. See Fig. 3-11 c). The pedestal in the resulting Unser spectrum is subtracted as described above. Since the RF cavity monitors are low noise devices, their spectra do not need to be background subtracted. The frequency distributions measured by the Unser and RF cavities in each current step are fitted with normal distributions, and the resulting means are taken to be the measured frequencies. The Unser gain parameter obtained in the Unser calibration step is then used to determine the beam current, which is plotted vs. the measured RF cavity frequency. This plot is fitted with a linear function 

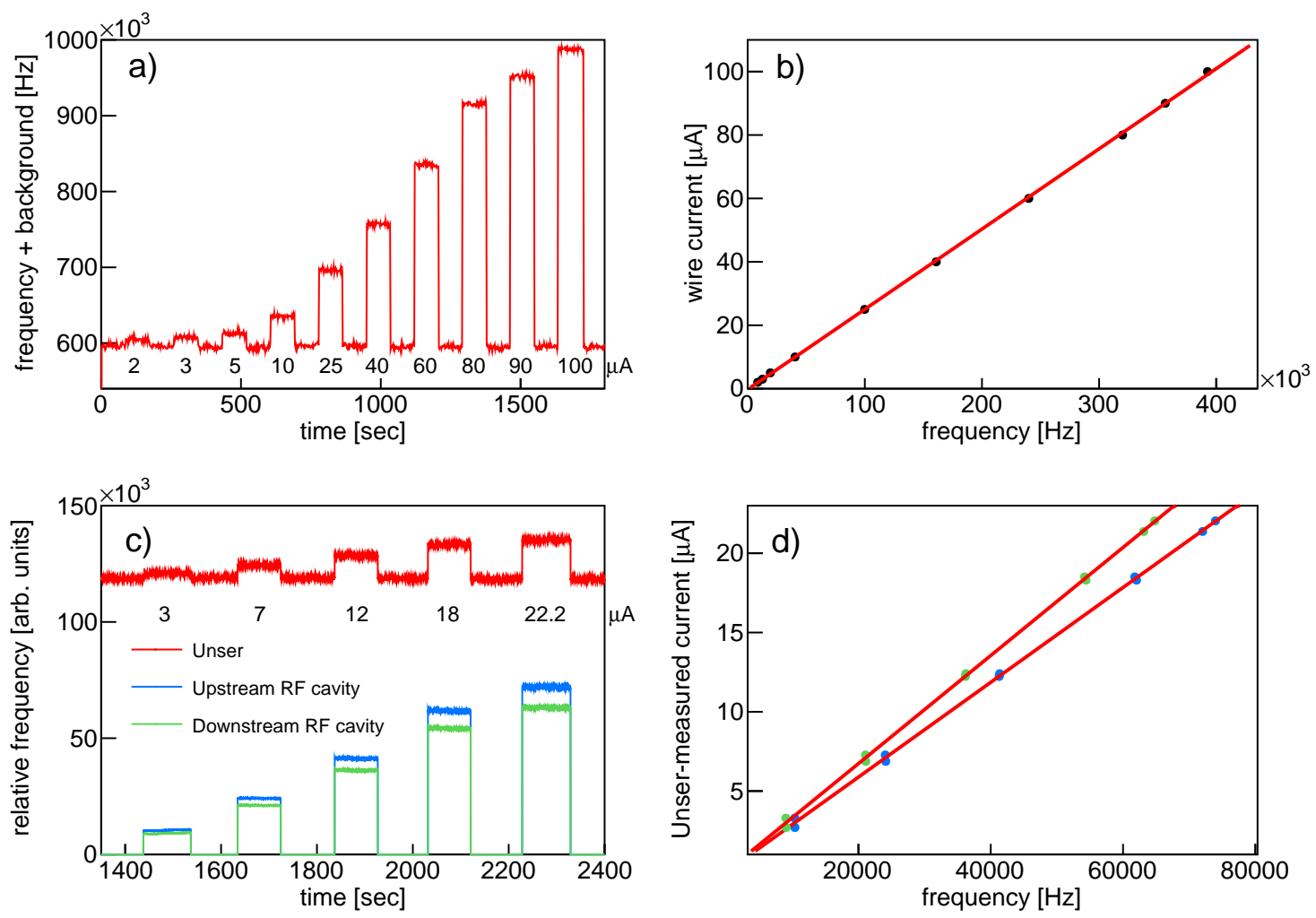

Figure 3-11: (Top) Unser calibration: a) frequency measured by the Unser as a function of time. The steps correspond to different values $(2,3,5,10,25,40,60,80,90,100 \mu \mathrm{A})$ of the input current passed through the Unser's internal wire. The lowest frequency values correspond to the pedestal measured when the wire current is set to zero. b) Input wire current as a function of the pedestal subtracted Unser frequency. A linear fit allows one to extract the Unser calibration parameters (gain and offset). (Bottom) RF cavity calibration: c) frequency measured by the Unser and RF cavity monitors as a function of time. The steps correspond to different values $(3,7,12,18,22.2 \mu \mathrm{A})$ of the electron beam current. The lowest frequency values in the Unser signal correspond to the pedestal measured when the beam current is set to zero (beam turned off). d) Beam current determined from the calibrated Unser as a function of RF cavity frequency. A linear fit allows one to extract the RF cavity calibration parameters (gain and offset, see Table 3.5). 
$I_{\text {Unser }}=\left(\operatorname{gain}_{R F}\right) \cdot f_{R F}+$ offset $_{R F}$. Finally, the RF cavity gain and offset are stored and used to determine the beam current in subsequent runs. See Table 3.5 and Fig. 3-11 d).

Table 3.5: RF-cavity calibration parameters. See text for details.

\begin{tabular}{c|cc} 
& $\operatorname{gain}_{R F}[\mu \mathrm{A} / \mathrm{Hz}]$ & offset $_{R F}[\mu \mathrm{A}]$ \\
\hline \hline Upstream RF cavity & $(2.96 \pm 0.03) \mathrm{e}-04$ & $-0.10 \pm 0.10$ \\
Downstream RF cavity & $(3.35 \pm 0.03) \mathrm{e}-04$ & $-0.09 \pm 0.10$
\end{tabular}

\subsubsection{HRS optics calibration}

Particles detected in the HRS travel about $23 \mathrm{~m}$ from the target to the spectrometer focal plane, where their coordinates are measured by the VDCs. We measured the spectrometer "optics" in order to be able to reconstruct the particle's coordinates at the target $(\delta, \theta, y, \phi)_{t g}$ from the measured coordinates at the focal plane $(x, \theta, y, \phi)_{f p}$. The reconstruction of coordinates at the target from the coordinates measured at the focal plane is described by an optics tensor $(\mathbb{T})$ [117]:

$$
\left[\begin{array}{l}
\delta \\
\theta \\
y \\
\phi
\end{array}\right]_{t g}=\mathbb{T}\left[\begin{array}{l}
x \\
\theta \\
y \\
\phi
\end{array}\right]_{f p} .
$$

The variables measured at the focal plane correspond to the position of the particle and the tangent of the angle made by its trajectory along $\left(x_{f p}, \theta_{f p}\right)$ and perpendicular to $\left(y_{f p}, \phi_{f p}\right)$ the dispersive direction. The variables reconstructed at the target $\left(\delta, y_{t g}, \phi_{t g}, \theta_{t g}\right)$ are related to physical variables (momentum, z-coordinate of the interaction vertex, and in- and out-of-plane angles). The elements of $\mathbb{T}$ are written as: 


$$
\begin{aligned}
y_{t g} & =\sum_{j, k, l} Y_{j k l} \theta_{f p}^{j} y_{f p}^{k} \phi_{f p}^{l}, \\
\theta_{t g} & =\sum_{j, k, l} T_{j k l} \theta_{f p}^{j} y_{f p}^{k} \phi_{f p}^{l}, \\
\phi_{t g} & =\sum_{j, k, l} P_{j k l} \theta_{f p}^{j} y_{f p}^{k} \phi_{f p}^{l}, \\
\delta & =\sum_{j, k, l} D_{j k l} \theta_{f p}^{j} y_{f p}^{k} \phi_{f p}^{l},
\end{aligned}
$$

where the tensors $Y_{j k l}, T_{j k l}, P_{j k l}$, and $D_{j k l}$ are polynomials in $x_{f p}$. For example:

$$
D_{j k l}=\sum_{i=0}^{m} C_{i}^{D_{j k l}} x_{f p}^{i} .
$$

The optimization method consists of finding the coefficients for these tensors that best reproduce known positions, angles, and momenta through a $\chi^{2}$-minimization procedure. There are well established codes that have been traditionally used for this task in Hall A. In principle, the optics tensor should be a property of the spectrometer which, once determined, could be indefinitely used. In practice, the spectrometers are complex systems with many moving components that are individually calibrated and from time to time replaced and, consequently, different experiments have to, at least, check the quality of the optics tensor at the time of the measurement.

The $Y, T$, and $P$ coefficients are determined by taking data with the optics (or multi-foil) target (see Fig. 3-4) and sieve-slit collimators (see Fig. 3-12). The optics target corresponds to 11 (0.25-mm-thick) carbon foils separated by $2.5 \mathrm{~cm}$ (thus spanning a total length of $25 \mathrm{~cm}$ ) with the central foil located at the center of the target ladder $(z=0)$. The sieve-slit collimators are stainless steel slabs with a pattern of 49 holes spaced differently in the horizontal and vertical directions. That, combined with the different-sized holes, allow for the unambiguous determination of the collimator orientation. The sieve-slit collimators are placed at the entrance of the spectrometers. The known $z$-coordinates of the foils in the optics target, and $x$ - and 
$y$-coordinates of the different holes in the sieve-slit collimator (in a given HRS) can be written as a function of $y_{t g}, \phi_{t g}$, and $\theta_{t g}$, and thus the $Y, T, P$ coefficients can be determined (for that HRS) from this measurement.
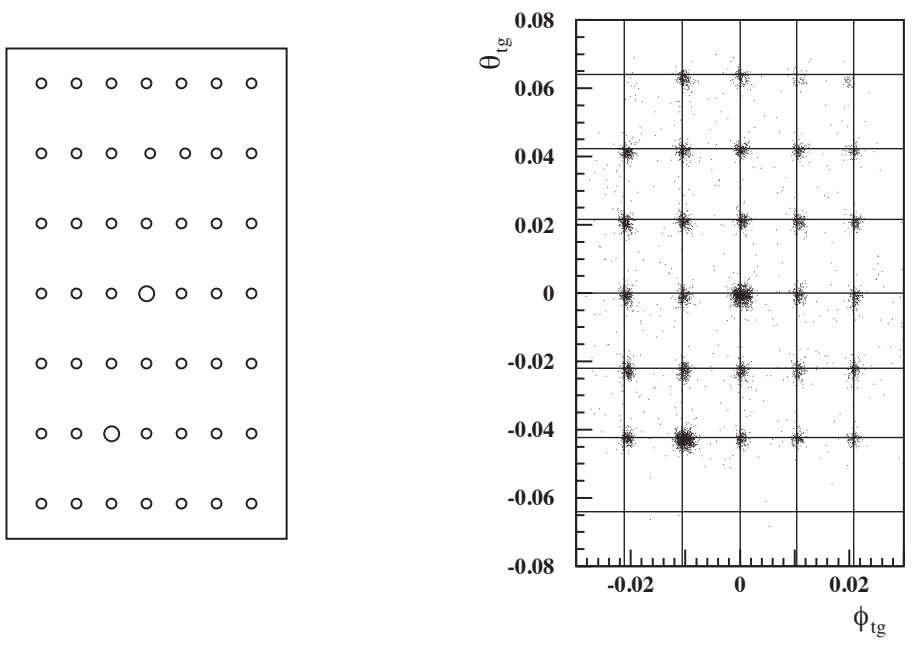

Figure 3-12: Sieve-slit collimator pattern: (left) geometric and (right) reconstructed.

The $D$ coefficients can be determined, for example, by measuring a kinematicallyoverconstrained reaction such as elastic $\mathrm{H}\left(e, e^{\prime}\right)$ in different momentum ranges and determining the parameters that minimize the difference between the momentum reconstructed from the measured $\delta$ and that reconstructed from the electron scattering angle.

The starting point for our optics optimization corresponded to the tensor coefficients determined by the JLab GMp experiment (E12-07-108), which preceded the series of Tritium experiments and thoroughly calibrated the Hall-A spectrometers [118]. The $Y, T$, and $P$ coefficients determined by the GMp experiment were re-optimized via the standard method with optics target and sieve-slit collimator data.

\section{High-order HRS optics check}

We checked the HRS optics performance by verifying that quantities with physical meaning are correctly reconstructed over the entire spectrometer acceptance. For example, the missing energy for $\mathrm{H}\left(e, e^{\prime} p\right)$ elastic scattering should be a delta function centered at zero. The 'narrowness' of this quantity makes it particularly sensitive to 
the HRS optics. While $E_{\text {miss }}$ did not vary with LHRS $y_{t g}, \phi_{t g}$, and $\delta$, or with any of the RHRS variables in our analysis, it did vary with LHRS $\theta_{t g}$ (see Fig. 3-13 left).
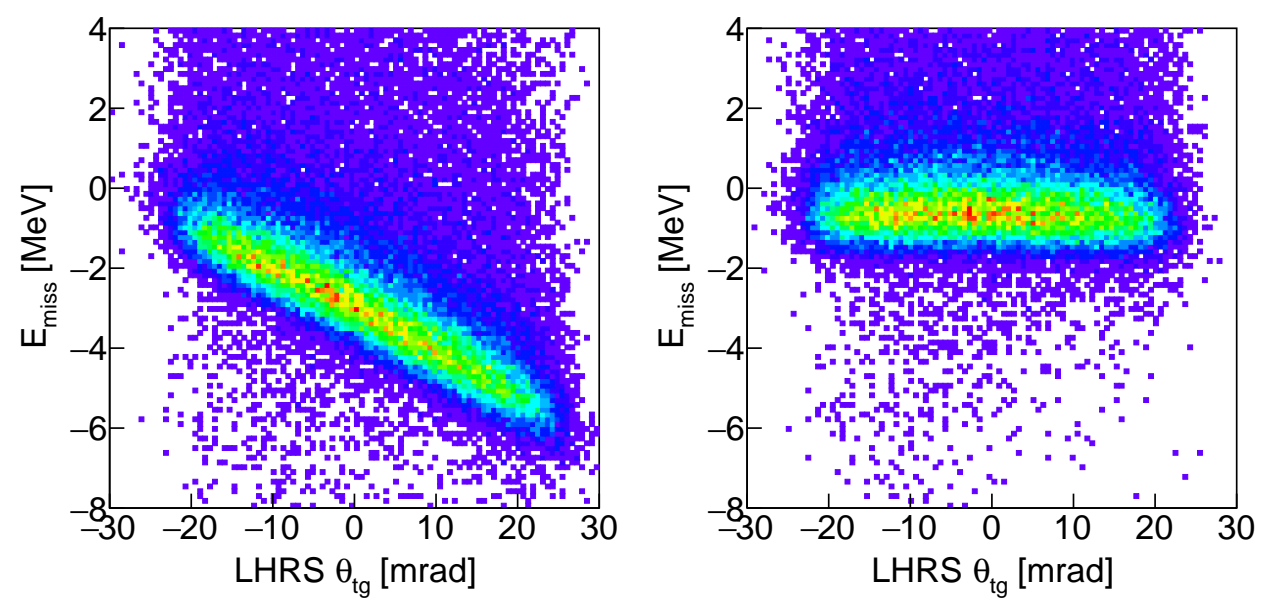

Figure 3-13: $E_{\text {miss }}$ dependence as a function of LHRS $\theta_{t g}$ for $\mathrm{H}\left(e, e^{\prime} p\right)$ elastic scattering. $E_{\text {miss }}$ should be a delta function centered at 0 , and should have no dependence on any kinematical variable. The left plot shows a linear 'optical aberration' between $E_{\text {miss }}$ and $\theta_{t g}$. The right plot shows the same histogram after correcting the LHRS matrix element $C_{0}^{D_{100}}$. We see that, after the correction, $E_{\text {miss }}$ is not centered around 0 yet (because the $0^{\text {th }}$-order corrections from the next section still need to be applied). Nevertheless, the optical aberration is not present anymore.

Since $E_{m i s s}$ is a function of $\delta$, not $\theta_{t g}$, any dependence of $E_{m i s s}$ in $\theta_{t g}$ can be interpreted as a spurious dependence between $\delta$ and $\theta_{t g}$. Since the 'optical aberration' is linear, the problem should come from the matrix element that relates $\delta$ and $\theta_{t g}$ linearly. Since $\theta_{t g} \propto \theta_{f p}$ (i.e. the only non-zero linear coefficient in equation 3.9 is $T_{100}$ ), we see from equations 3.11 and 3.12 that this element is $C_{0}^{D_{100}}$. We fitted this slope and extracted a correction equal to:

$$
\Delta C_{0}^{D_{100}}=(7.36 \pm 0.08) \times 10^{-2}
$$

The right plot in Fig. $3-13$ shows the corrected dependence between $E_{\text {miss }}$ and $\theta_{t g}$. This was the only high-order 'optical aberration' found. See Appendix A Figs. A-1 through A-10 for plots of several physical quantities as a function of different spectrometer variables after this correction is applied. Although some of these distributions are broader than $E_{\text {miss }}$, all of them exhibit the expected 'flat' behavior within 
approximately $1 \mathrm{MeV}$.

\section{$0^{\text {th }}$-order HRS optics check}

Elastic $\mathrm{H}\left(e, e^{\prime} p\right)$ has only two degrees of freedom. Consequently, once any pair of variables is fixed, the entire kinematics of the interaction are determined. This makes elastic $\mathrm{H}\left(e, e^{\prime} p\right)$ a powerful tool to check optics calibrations. For example, we can write the beam energy as a function of the electron and proton scattering angles as:

$$
E_{e}=m_{p}\left(\cot \frac{\theta_{e^{\prime}}}{2} \cot \theta_{p}-1\right)
$$

Alternatively, we can write the scattered electron momentum as a function of the beam energy and electron scattering angle as:

$$
\left|\vec{p}_{e^{\prime}}\right| \approx E_{e^{\prime}}=\frac{m_{p} E_{e}}{m_{p}+E_{e}\left(1-\cos \theta_{e^{\prime}}\right)}
$$

We can combine these expressions to write, for example, $\theta_{p}\left(E_{e}, p_{e^{\prime}}\right), \theta_{p}\left(p_{e^{\prime}}, \theta_{e^{\prime}}\right)$, or $\theta_{p}\left(E_{e}, \theta_{e^{\prime}}\right)$ (or any other pair of independent variables). The difference between any of these distributions and $\theta_{p}$ measured by the RHRS, should be a normal distribution centered at zero. However, this was not the case (see Fig. 3-14 left). The fact that all three distributions are centered at the same value is an indication that only the measured $\theta_{p}$ is off. We fitted gaussians to these three distributions, and took their weighed average as a correction factor. Subsequently, this offset was expressed in terms of the corresponding RHRS optics variable and added to the optics tensor tables that are used to process the data. This correction was introduced as the following optics offset:

$$
\Delta C_{0}^{P_{000}}=(-1.567 \pm 0.008) \times 10^{-3}
$$

This corresponds to a 1.6 mrad offset in the spectrometer central angle. The corrected plot is shown in Fig. 3-14 right.

The same problem was seen when studying the proton momentum. The correction 

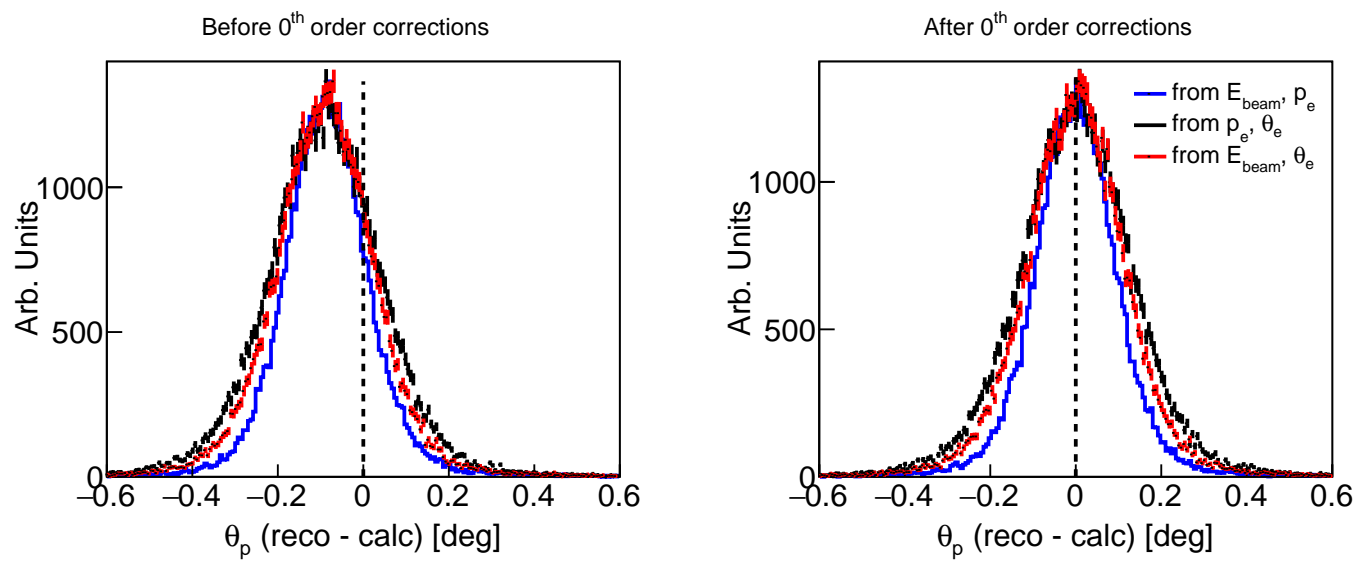

Figure 3-14: Difference between proton scattering angle measured by RHRS (reco), and calculated from different pairs of variables (calc). The plot on the left shows the distributions before any '0th' order correction. The plot on the right shows the status after all the corrections described in this section. All the distributions have been normalized to have the same height at their mean value.

introduced to the RHRS optics matrix was:

$$
\Delta C_{0}^{D_{000}}=(-1.202 \pm 0.007) \times 10^{-3},
$$

corresponding to a small offset in the spectrometer central momentum. A similar problem in the missing momentum $y$-component was fixed with the following correction in the RHRS optics matrix:

$$
\Delta C_{0}^{T_{000}}=(-2.940 \pm 0.009) \times 10^{-3} .
$$

See Appendix AFigs. A-11 through A-17 for plots showing additional distributions before and after these ' ${ }^{t h}$ ' order corrections. After these corrections, the $\mathrm{H}\left(e, e^{\prime} p\right) p_{\text {miss }}$ resolution was better than $9 \mathrm{MeV} / c$.

\section{Final optics checks from $\mathbf{d}\left(e, e^{\prime} p\right)$ QE scattering}

To check the validity of all the aforementioned optics corrections, we looked at their effect on $\mathrm{d}\left(e, e^{\prime} p\right)$ QE distributions measured in the low- $p_{\text {miss }}$ kinematical setting. Fig. 3-15 shows the missing-energy distributions before and after the optics corrections. There are two clear features in these plots. First, we can see the effect from 
correcting the LHRS $C_{0}^{D_{100}}$ (linear) element as the resolution enhancement from the blue to the red curves. Second, we can see the effect from correcting the RHRS $0^{\text {th }}$ order elements as the shift of the distributions towards their expected values.

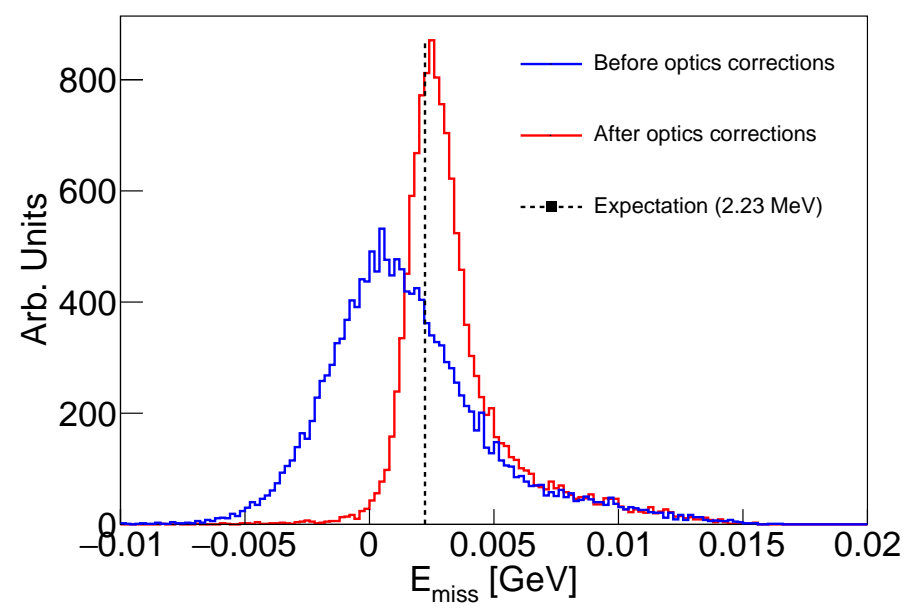

Figure 3-15: Missing energy in $\mathrm{d}\left(e, e^{\prime} p\right)$ QE scattering before and after optics corrections.

\subsection{Luminosity check}

In order to verify the absolute normalization of the measured distributions, we compared elastic $\mathrm{H}\left(e, e^{\prime} p\right)$ distributions to a simple yield-estimate calculation. The number of detected events per unit time can be written as:

$$
\frac{\Delta N}{\Delta t}=\mathscr{L} \sigma \Delta \Omega,
$$

where $\sigma$ is the interaction cross section, $\mathscr{L}=I \cdot T$ is the luminosity, and $\Delta \Omega$ is the covered solid angle. Here, $I$ refers to the beam current in units of electrons/second, and $T$ corresponds to the number of protons per unit $\mathrm{cm}^{2}$ :

$$
T=\frac{\rho \cdot l \cdot N_{A} \cdot n_{p p m}}{M},
$$

where $\rho=2.832 \mathrm{mg} / \mathrm{cm}^{3}$ is the target density, $N_{A}$ is Avogadro's number, $M=2.016$ $\mathrm{g} / \mathrm{mol}$ is the molar mass (grams per mol of $\mathrm{H}_{2}$ molecule), $n_{p p m}=2$ is the number of 
protons per $H_{2}$ molecule, and $l$ is the effective target length.

The elastic scattering of electrons off a hydrogen target is described by the unpolarized differential cross section known as the Rosenbluth cross section:

$$
\begin{aligned}
\frac{d \sigma}{d \Omega} & =\left.\frac{d \sigma}{d \Omega}\right|_{M o t t}\left(\frac{1}{1+\tau}\right)\left[G_{E}^{2}\left(Q^{2}\right)+\frac{\tau}{\epsilon} G_{M}^{2}\left(Q^{2}\right)\right] \\
\tau & =Q^{2} /\left(4 m_{p}^{2}\right) \\
\epsilon^{-1} & =1+2(1+\tau) \tan ^{2}\left(\theta_{e^{\prime}} / 2\right)
\end{aligned}
$$

where $G_{E}$ and $G_{M}$ are respectively the electric and magnetic proton Sachs form factors. $d \sigma /\left.d \Omega\right|_{\text {Mott }}$ is the differential Mott cross section corrected for proton recoil:

$$
\left.\frac{d \sigma}{d \Omega}\right|_{M o t t}=\frac{\alpha^{2} E_{e^{\prime}}}{4 E_{e}^{3} \sin ^{4} \theta_{e^{\prime}} / 2} \cos ^{2} \frac{\theta_{e^{\prime}}}{2}
$$

where $\alpha$ is the fine structure constant, $E_{e}$ is the beam energy, and $E_{e^{\prime}}$ is the scatteredelectron energy. This expression is then integrated over $\phi_{e^{\prime}}$ :

$$
\frac{d \sigma_{M o t t}}{\sin \theta_{e^{\prime}} d \theta_{e^{\prime}}}=\int_{\phi_{e^{\prime}, 0} / \sin \theta_{e^{\prime}}}^{\phi_{e^{\prime}, f} / \sin \theta_{e^{\prime}}} \frac{\alpha^{2} E_{e^{\prime}}}{4 E_{e}^{3} \sin ^{4} \theta_{e^{\prime}} / 2} \cos ^{2} \frac{\theta_{e^{\prime}}}{2} d \phi_{e^{\prime}}
$$

where, since the spectrometers cover a constant solid angle $d \Omega=\sin \theta_{e^{\prime}} d \theta_{e^{\prime}} d \phi_{e^{\prime}}$, the $\phi_{e^{\prime}}$ limit depends on $\theta_{e^{\prime}}$. This implies:

$$
\frac{d \sigma_{M o t t}}{d \theta_{e^{\prime}}}=\frac{\alpha^{2} E_{e^{\prime}}}{4 E_{e}^{3} \sin ^{4} \theta_{e^{\prime}} / 2} \cos ^{2} \frac{\theta_{e^{\prime}}}{2}\left(\phi_{e^{\prime}, f}-\phi_{e^{\prime}, 0}\right)
$$

We chose $\phi_{e^{\prime}}$ to be well within the spectrometer acceptance edges $\left(\left|\phi_{e^{\prime}}\right|<30 \mathrm{mrad}\right)$. Finally, we multiply this result by $(\hbar c)^{2}$ to get the correct units.

Radiative effects were included by multiplying by $\left(1+\delta\left(\theta_{e^{\prime}}, \Delta E\right)\right)$, where $\delta\left(\theta_{e^{\prime}}, \Delta E\right)$ is taken from the formalism developed by Mo and Tsai [119]. Only events between the elastic peak $E_{e^{\prime}}=m_{p} E_{e} /\left(m_{p}+E_{e}\left(1-\cos \theta_{e^{\prime}}\right)\right)$ and $E_{e^{\prime}}-\Delta E$ are included in both the estimate and the data sample. In this study, we selected a constant $\Delta E \approx 65 \mathrm{MeV}$, as shown on the left of Fig. 3-16. 

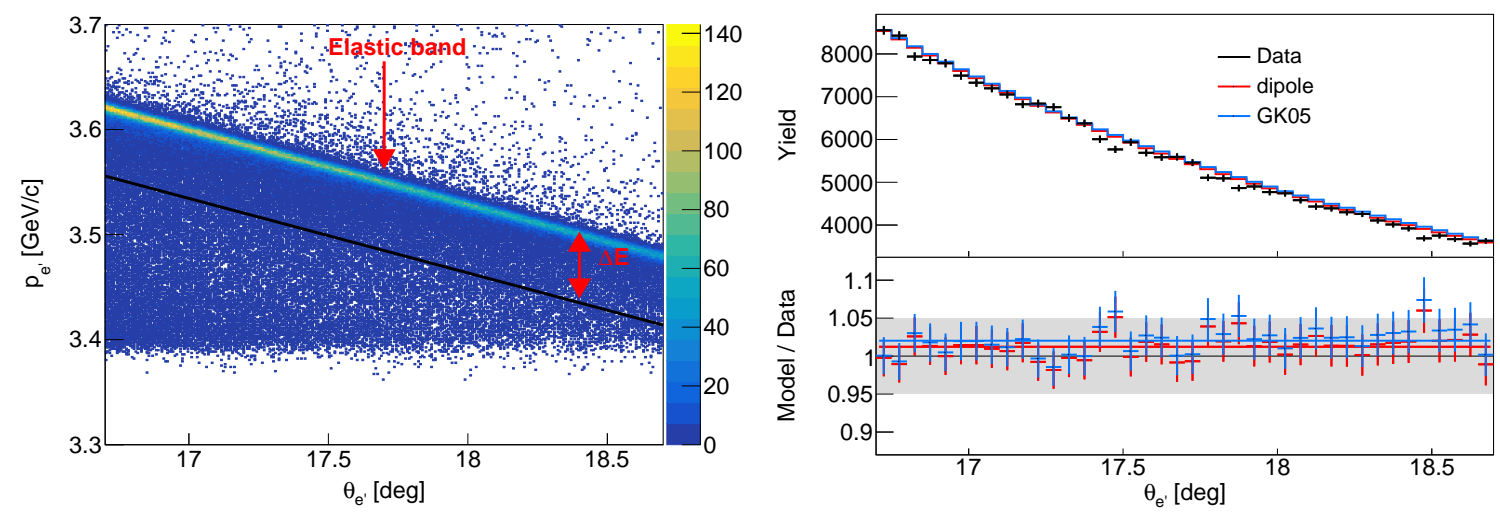

Figure 3-16: (Left) electron momentum as a function of electron scattering angle. The diagonal black line marks the choice of lower limit for the selected window $\Delta E \approx 65 \mathrm{MeV}$ used in the calculation of radiative effects. (Right) comparison between measured and calculated yields as a function of electron scattering angle. The calculation was carried out with the form factor model from [120] (blue) and also using the dipole parametrization (red). The two calculations agree with the data within $2 \%$.

Finally, before comparing to the yield-estimate calculation, the measured data was corrected for variations in the target density caused by local temperature fluctuations (boiling), and dead time (the fraction of time in which the DAQ cannot record data). The results are shown on the right of Fig. 3-16. The calculations were carried out both with the proton form factors taken from [120] and also with the traditional dipole parametrization: $G_{D}\left(Q^{2}\right)=1 /\left(1+\frac{Q^{2}}{0.71\left(\mathrm{GeV} / c^{2}\right)^{2}}\right)^{2}$. The calculations agree with the measurement within $2 \%$.

\subsection{Data analysis}

\subsubsection{Event selection}

We histogrammed the total number of detected events in each run normalized to the beam charge, and discarded a small number of runs for which this quantity was more than $3 \sigma$ away from the mean. Only events with a single track reconstructed in each spectrometer were kept. Finally, we only kept events for which the beam current was within $\pm 1.5 \mu \mathrm{A}$ of the average value to exclude unwanted beam interruptions, commonly referred to as beam trips. The event-selection cuts described below were 
applied to the resulting sample.

\section{Acceptance and vertex cuts}

As acceptance-edge effects are very difficult to model and quantify, we only considered events for which electrons and protons were detected within $\pm 4 \%$ of the central spectrometer momentum $(\delta)$, and $\pm 27.5 \mathrm{mrad}$ in in-plane $\left(\phi_{t g}\right)$ and $\pm 55.0 \mathrm{mrad}$ in out-of-plane angle $\left(\theta_{t g}\right)$ relative to the center of the spectrometer acceptance. See Table 3.2 for reference. The effect of these cuts is shown in Fig. 3-17.
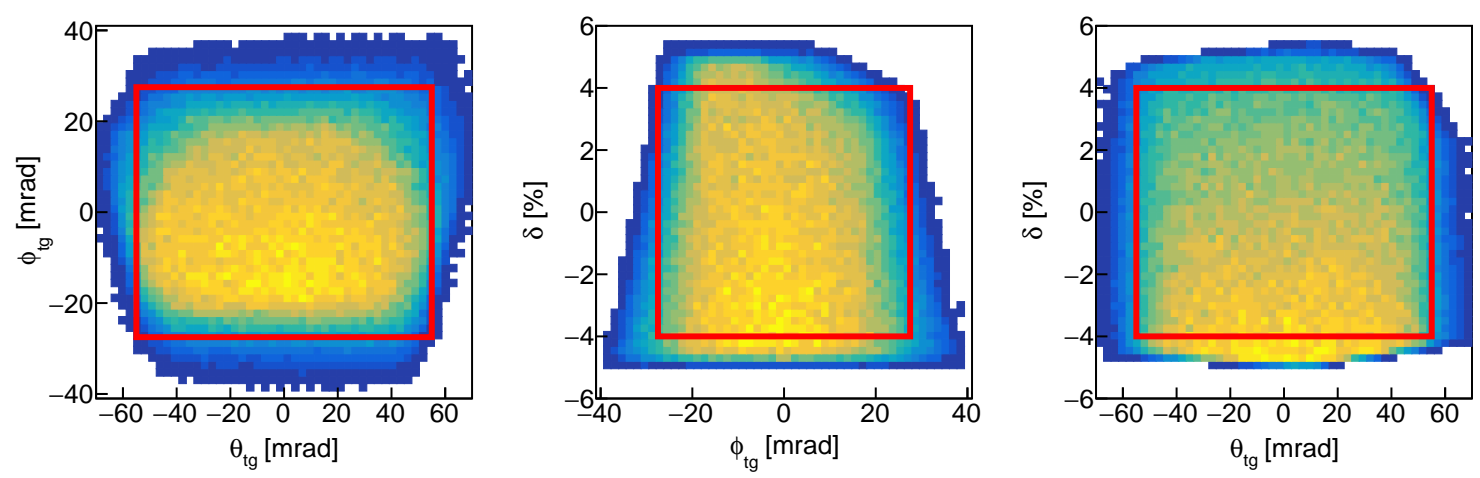

Figure 3-17: HRS acceptance from a SIMC simulation and effect of acceptance cuts. The plots correspond to $\phi_{t g}$ vs $\theta_{t g}$ (left), $\delta$ vs $\phi_{t g}$ (center), and $\delta$ vs $\theta_{t g}$ (right). The red boxes represent the applied acceptance cuts.

The interaction vertex $z$-component, which is approximately related to $y_{t g}$ as:

$$
z=\frac{y_{t g}}{\sin \theta_{c}}
$$

where $\theta_{c}$ is the HRS central angle, was required to originate within the central $\pm 9 \mathrm{~cm}$ of the 25-cm gas-target cell to exclude events originating from the target walls. By measuring scattering from the empty cell described in section 3.1.3, we determined that the target cell wall contribution to the measured $\left(e, e^{\prime} p\right)$ event yield was negligible $(\ll 1 \%)$.

Coincidence events were selected by requiring the LHRS and RHRS z-component of the reconstructed vertices to be within $\pm 1.2 \mathrm{~cm}$ of each other, which corresponds to $\pm 3 \sigma$ of the vertex reconstruction resolution. 
Fig. 3-18 shows the $z$-component of the interaction vertex reconstructed with the LHRS vs RHRS. The black points correspond to events before vertex cuts for the low$p_{m i s s}$ kinematical setting for the ${ }^{3} \mathrm{He}$ and empty targets. The equivalent plots for the high- $p_{\text {miss }}$ kinematical setting are similar. Additionally, for both settings, the ${ }^{3} \mathrm{H}$ plots are similar to the ${ }^{3} \mathrm{He}$ ones. The horizontal clusters of events at $\pm 12.5 \mathrm{~cm}$, present in both plots, correspond to the downstream and upstream caps of the aluminum target cell respectively. For these events, an electron scatters of the aluminum cap and goes into the LHRS acceptance, and the RHRS vertex is reconstructed to a random position. The pronounced diagonal band, only present in the ${ }^{3} \mathrm{He}$ plot, corresponds to coincidence events in which the vertex is reconstructed by both spectrometers to approximately the same position. Finally, the remaining events scattered throughout both plots correspond to random coincidence events. Due to the low experimental luminosity, the random coincidence event rate was negligible $(\ll 1 \%)$. The width of the diagonal band is determined by the $3 \sigma$ coincidence vertex cut, and its length is defined by the $\pm 9 \mathrm{~cm}$ cut.
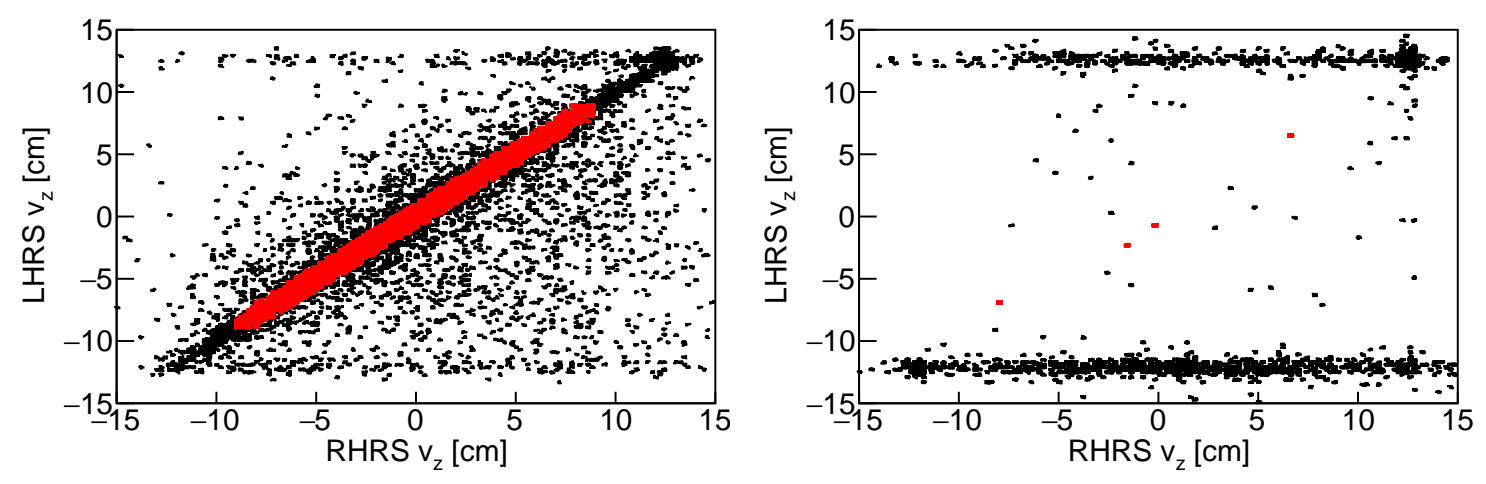

Figure 3-18: $z$-component of the interaction vertex reconstructed with the LHRS vs RHRS before and after vertex cuts in the low- $p_{\text {miss }}$ kinematical setting in the case of the ${ }^{3} \mathrm{He}$ (left) and empty (right) targets. The red points correspond to events after the vertex cuts described in the text.

\section{Particle identification (PID) cuts}

Electrons were identified by the ratio of their energy measured in the calorimeter to their momenta. Other negative particles (such as pions) will only deposit a fraction of their energy in the calorimeter and, as a result, the $E_{\text {cal }} /|\vec{p}|$ ratio will be smaller 
than that for electrons. We identified electrons by requiring $E_{\text {cal }} /\left|\vec{p}_{e}\right|>0.5$. See left plot in Fig. 3-19.
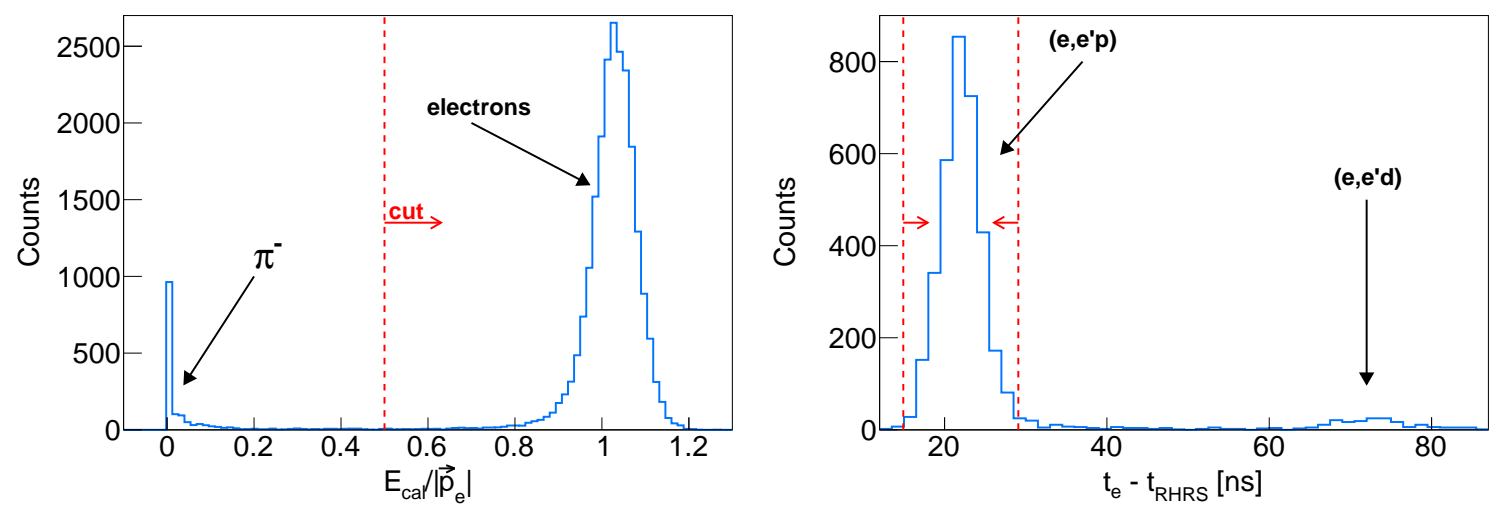

Figure 3-19: Particle-identification criteria. Left: electron PID. Electron candidates are required to deposit more than $50 \%$ of their energy in the calorimeter (i.e. $\left.E_{\text {cal }} /\left|\vec{p}_{e}\right|>0.5\right)$. Right: proton PID. Protons are selected based on their relative time with respect to the corresponding electron.

We identified coincident protons in the RHRS by their arrival time with respect to the electron in the LHRS. The long optical path from the target to the scintillator detectors, which form the trigger, causes different-mass particles to create coincidence events well separated in time, which can be used for PID. For example, the travel time difference between $1.48 \mathrm{GeV} / c$ protons and deuterons over a 25 -m path gives a difference in time between $\left(e, e^{\prime} p\right)$ and $\left(e, e^{\prime} d\right)$ events of $\approx 50 \mathrm{~ns}$. The right plot in Fig. 3-19 shows the measured distribution for the difference in time between the electron and the RHRS event triggers. The two visible bumps correspond to $\left(e, e^{\prime} p\right)$ and $\left(e, e^{\prime} d\right)$ events. Proton coincidence events were selected by placing a $\pm 3 \sigma$ cut around the $\left(e, e^{\prime} p\right)$ peak, as shown by the red dashed lines.

\section{Physics cuts}

In addition to the cuts described above, we also required that $\theta_{r q}<37.5^{\circ}$, and (for high- $p_{\text {miss }}$ kinematics) $x_{B}>1.3$. These cuts should minimize the effect of non-QE reaction mechanisms (see section 2.2.2). 


\subsubsection{Data-SIMC comparison}

After all these cuts were applied, the resulting measured and simulated distributions were compared. Fig. 3-20 shows the number of ${ }^{3} \mathrm{H}\left(e, e^{\prime} p\right)$ events (counts) as a function of missing energy (and $Q^{2}$ in the insert) for the low- $p_{m i s s}$ kinematical setting. The equivalent ${ }^{3} \mathrm{He}\left(e, e^{\prime} p\right)$ distributions and the distributions of other kinematical variables can be found in Appendix B. The simulation describes the shapes of the measured distributions well enough to be used in the data analysis as described in the following sections.

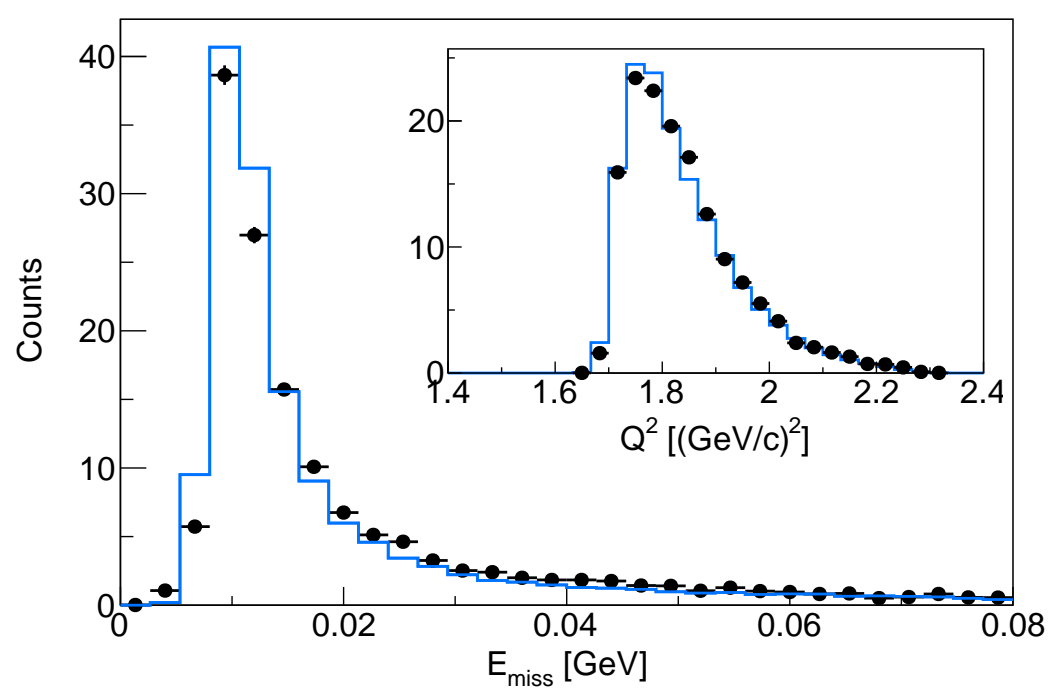

Figure 3-20: Comparison between ${ }^{3} \mathrm{H}\left(e, e^{\prime} p\right)$ measured and simulated distributions for the low- $p_{\text {miss }}$ kinematical setting. The simulated distributions are normalized to give the same integral as the data. See Appendix B for additional distributions. The black markers correspond to the measured distribution, while the blue lines correspond to the SIMC simulation with the $\mathrm{CK}+C C 1$ model (normalized to give the same integral as the data).

\section{Effect of interaction between the two spectator nucleons $\left(\mathrm{FSI}_{23}\right)$}

The PWIA cross-section calculations can be carried out either including or excluding the continuum interaction between the two unstruck nucleons in the $A=3\left(e, e^{\prime} p\right)$ reaction (see diagram b) in Fig. 2-3). To assess the importance of this effect, we compared the missing-energy distributions from simulations carried out with and without 
the inclusion of this diagram. The results are shown in Fig. 3-21. FSI $_{23}$ is nonnegligible and is more important in the low- $p_{\text {miss }}$ kinematical setting. Consequently, we only used calculations that do not include $\mathrm{FSI}_{23}$ in the high- $p_{\text {miss }}$ kinematical setting.
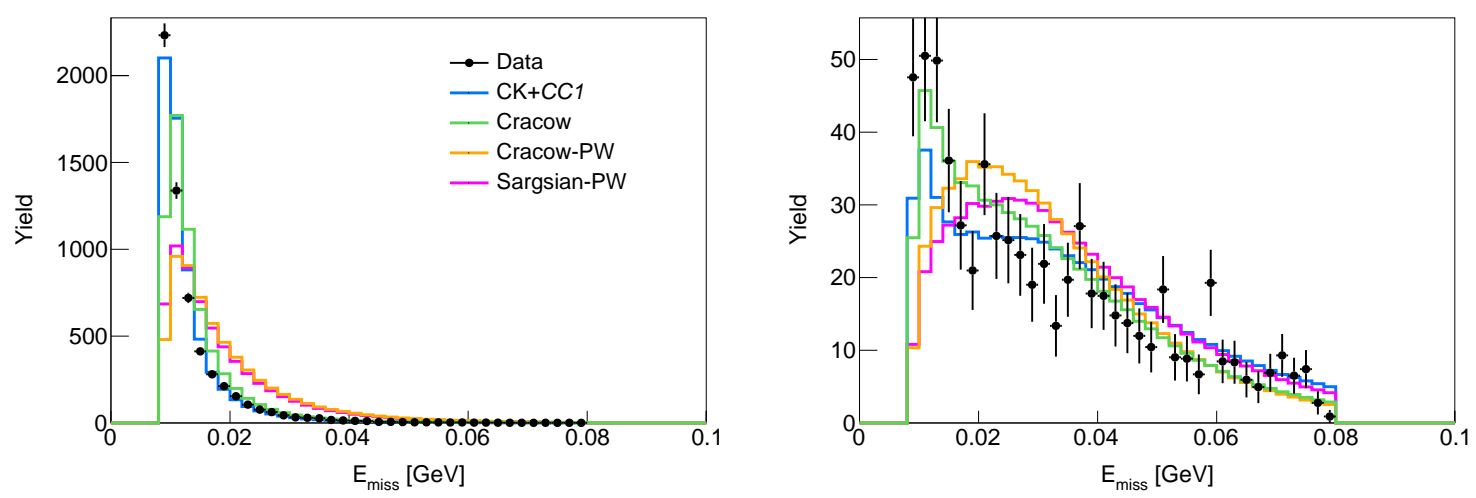

Figure 3-21: Comparison between ${ }^{3} \mathrm{He} E_{\text {miss }}$ distributions from data and simulations with $\left(\mathrm{CK}+C C 1\right.$ and Cracow) and without (Cracow-PW and Sargsian-PW) $\mathrm{FSI}_{23}$ for the low- $p_{\text {miss }}$ (left) and high- $p_{\text {miss }}$ (right) kinematical settings. The $\mathrm{FSI}_{23}$ effect is more important in the low- $p_{\text {miss }}$ kinematical setting. The corresponding ${ }^{3} \mathrm{H}$ plot leads to the same results.

In the remainder of this chapter, I will describe two separate but related analyses. The first one corresponds to the direct extraction of the ${ }^{3} \mathrm{He} /{ }^{3} \mathrm{H}\left(e, e^{\prime} p\right)$ cross-section ratio [101. The second one corresponds to the extraction of the ${ }^{3} \mathrm{He}$ and ${ }^{3} \mathrm{H}\left(e, e^{\prime} p\right)$ absolute cross sections [102].

\section{6 ${ }^{3} \mathrm{He} /{ }^{3} \mathrm{H}\left(e, e^{\prime} p\right)$ cross-section ratio}

For each measured nucleus $A$ we binned the data in bins of $p_{\text {miss }}$ and calculated the normalized $\left(e, e^{\prime} p\right)$ yield:

$$
Y_{\mathrm{A}}\left(p_{\text {miss }}\right)=\frac{N\left(p_{\text {miss }}\right)}{C\left(\frac{\rho}{A} \cdot b\right) \cdot t_{\text {live }}},
$$

where $N\left(p_{\text {miss }}\right)$ is the number of counts detected in each $p_{\text {miss }}$ bin (integrated over the experimental $E_{\text {miss }}$ acceptance), $C$ is the delivered beam charge, $\rho$ is the target areal density (see Table 3.1), $b$ is the density correction (see section 3.6.1), and $t_{\text {live }}$ 
is the fraction of the time in which the DAQ is able to record data.

We calculated the ratio of the ${ }^{3} \mathrm{He}$ and ${ }^{3} \mathrm{H}$ normalized yields and then corrected it for the radioactive decay of ${ }^{3} \mathrm{H}$ (see section 3.6.2). We then corrected the yield ratios for radiative, bin-migration, and $E_{\text {miss }}$-acceptance effects (see sections 3.6.3, 3.6.4. and 3.6 .5 respectively).

\subsubsection{Density ('boiling') corrections}

The gas cells are filled with the gas densities specified in Table 3.1. However, local temperature fluctuations created by electron beam heating changes the gas density in its path. The density changes depend on the beam current and this effect needs to be taken into account when extracting yields and cross sections. The correction factor is determined by extracting the normalized $\left(e, e^{\prime}\right)$ yield:

$$
Y_{\mathrm{A}}^{\left(e, e^{\prime}\right)}(I)=\frac{N(I)}{C \cdot t_{\mathrm{live}} \cdot \epsilon},
$$

where $I$ is the beam current, $N(I)$ is the number of good electron events for a given beam current, $C$ is the delivered beam charge, $t_{\text {live }}$ is the fraction of the time in which the DAQ recorded data, and $\epsilon$ is the detector efficiency (tracking, PID, trigger, ...). In the absence of beam the density should correspond to its nominal value (Table 3.1. The yields are normalized such that $Y_{\mathrm{A}}^{\left(e, e^{\prime}\right)}(0 \mu \mathrm{A})=1$. Additional information on the determination of these corrections, including the event-selection criteria, check of the methodology on a solid target, efficiency studies, and other details can be found in [109]. The corrections used in this analysis for the different gas targets at $I=22.5 \mu \mathrm{A}$ are shown in Table 3.6 .

Table 3.6: Density correction factors at $I=22.5 \mu \mathrm{A}$ from 109 .

\begin{tabular}{c|c} 
Target & correction factor $(b)$ \\
\hline \hline${ }^{3} \mathrm{H}$ & $0.901 \pm 0.003$ \\
${ }^{2} \mathrm{H}(\mathrm{d})$ & $0.908 \pm 0.003$ \\
${ }^{1} \mathrm{H}$ & $0.884 \pm 0.004$ \\
${ }^{3} \mathrm{He}$ & $0.938 \pm 0.002$
\end{tabular}




\subsection{2 ${ }^{3} \mathrm{H}$-decay correction}

Tritium decays radioactively via the process ${ }^{3} \mathrm{H} \rightarrow{ }^{3} \mathrm{He}+e^{-}+\bar{\nu}_{e}+18.6 \mathrm{keV}$. Consequently, the ${ }^{3} \mathrm{H}$ density decreases with time according to:

$$
n_{{ }^{3} \mathrm{H}}(t)=n_{3}^{0} e^{-t / \tau_{3} \mathrm{H}},
$$

where $n_{3 \mathrm{H}}^{0}$ is the initial ${ }^{3} \mathrm{H}$ density in the target cell, and $\tau_{3} \mathrm{H}=(4500 \pm 8$ days $) / \ln (2)$ is the ${ }^{3} \mathrm{H}$ lifetime [121]. This process increases the ${ }^{3} \mathrm{He}$ fraction in the target cell by:

$$
n_{3 \mathrm{He}}(t)=n_{3 \mathrm{He}}^{0}+n_{3_{\mathrm{H}}}^{0}\left(1-e^{-t / \tau_{3} \mathrm{H}}\right),
$$

where $n_{3 \mathrm{He}}^{0}$ is the initial ${ }^{3} \mathrm{He}$ density in the target cell. Since the experiment took place between $t=171-195$ days after the ${ }^{3} \mathrm{H}$ cell was filled, and taking $\epsilon_{0} \equiv$ $n_{3_{\mathrm{He}}}^{0} /\left(n_{{ }^{\mathrm{He}} \mathrm{He}}^{0}+n_{3_{\mathrm{H}}}^{0}\right)=0.059 \%$ from [107], we find that the ${ }^{3} \mathrm{He}$ fraction present in the ${ }^{3} \mathrm{H}$ target at the time of our experiment was:

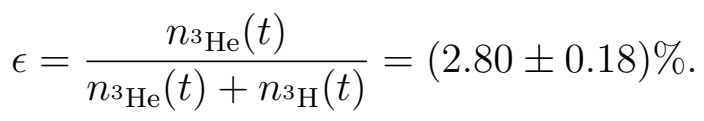

Thus, the cross section measured with the tritium cell $\left(\sigma_{3^{\mathrm{H}}}^{\mathrm{mixed}}\right)$ is a mixture of the tritium $\left(\sigma_{3} \mathrm{H}\right)$ and helium-3 $\left(\sigma_{3 \mathrm{He}}\right)$ cross sections:

$$
\sigma_{3 \mathrm{H}}^{\operatorname{mixed}}=(1-\epsilon) \sigma_{3_{3} \mathrm{H}}+\epsilon \sigma_{3} \mathrm{He} .
$$

Solving for $\sigma_{3} \mathrm{H}$ we get:

$$
\sigma_{3} \mathrm{H}=\frac{\sigma_{3^{\mathrm{H}}}^{\text {mixed }}-\epsilon \sigma_{3} \mathrm{He}}{1-\epsilon} .
$$

The ${ }^{3} \mathrm{H}$ decay correction is:

$$
R_{{ }^{3} \mathrm{He} /{ }^{3} \mathrm{H}}^{\text {corr.yeld }}=\frac{(1-\epsilon) R_{3^{\mathrm{He}} /{ }^{3} \mathrm{H}}^{\text {measured }}}{1-\epsilon R_{{ }^{3} \mathrm{He} /{ }^{3} \mathrm{H}}^{\text {measured }}},
$$

where $R_{3 \mathrm{He} /{ }^{3} \mathrm{H}}^{\text {measured }} \equiv \sigma^{3} \mathrm{He} / \sigma_{3{ }^{3} \mathrm{H}}^{\text {mixed }}$ and $R_{3^{3} \mathrm{He} /{ }^{3} \mathrm{H}}^{\text {corr }} \equiv \sigma^{3} \mathrm{He} / \sigma_{3} \mathrm{H}$ are the measured and decaycorrected cross-section ratios respectively. Fig. 3-22 shows the missing-momentum 
dependence of the corrected event yield ratio $R_{{ }^{3} \mathrm{He} /{ }^{3} \mathrm{H}}^{\text {corry }}$ in each kinematical setting. The ${ }^{3} \mathrm{He} /{ }^{3} \mathrm{H}$ ratio is about three at the smallest measured $p_{\text {miss }}$ and decreases to about 1.5 at $p_{\text {miss }} \approx 250 \mathrm{MeV} / c$, with a possible rise after that.

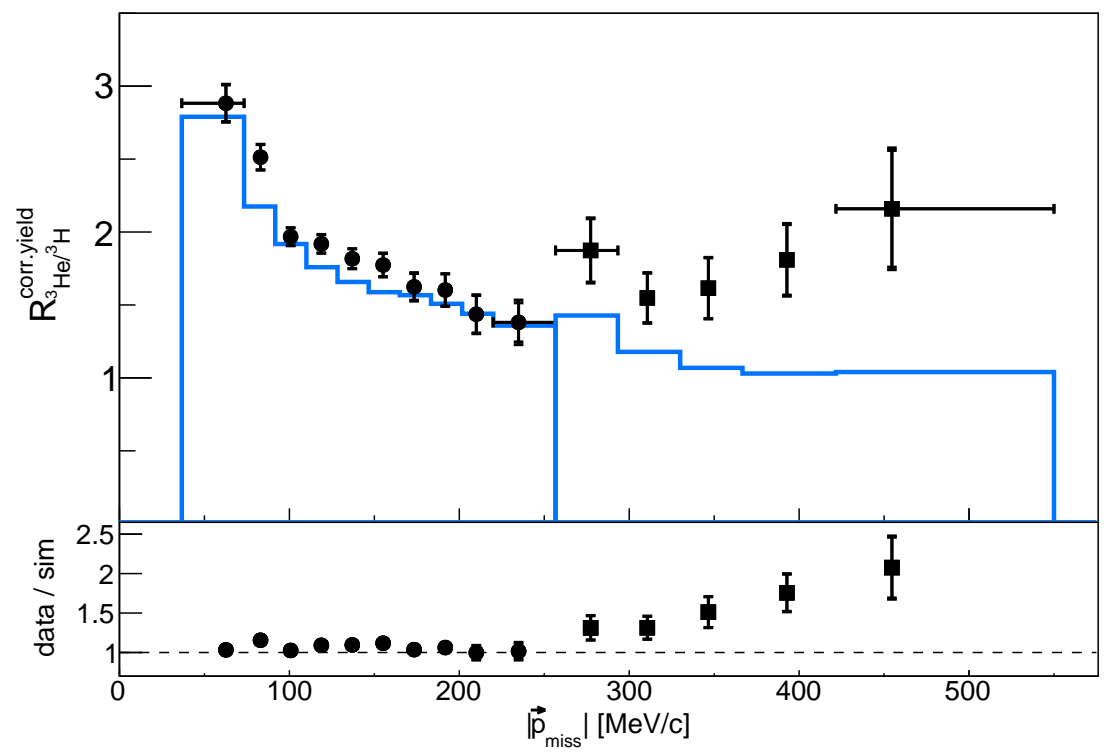

Figure 3-22: Missing-momentum dependence of the measured ${ }^{3} \mathrm{H}$-decay-corrected ${ }^{3} \mathrm{He} /{ }^{3} \mathrm{H}$ normalized event-yield ratios for the low- $p_{\text {miss }}(\bullet)$ and high- $p_{\text {miss }}(\boldsymbol{\square})$ kinematical settings. The error bars include both statistical and point-to-point systematical uncertainties (see Appendix C Table C.5. An additional overall normalization uncertainty is not shown (see Table 3.8). The solid histogram shows the PWIA SIMC simulation using equation 2.5 and the spectral function of [69]. The bin widths are the same for the histogram and the data.

\subsubsection{Radiative corrections}

We want to study the QE interaction between an electron and a proton embedded in the nuclear medium through the exchange of a single virtual photon, resulting in the emission of the struck nucleon. The diagram describing this process, presented in Fig. 3-23, is the leading order term (first-order Born approximation) in a perturbative expansion in powers of the fine structure constant $\alpha$.

Unavoidably, this is not the only process which contributes to the measured cross section. In the presence of the nuclear Coulomb field, electrons lose energy by the emission of real photons. This process is called "braking" or "Bremsstrahlung" radi- 


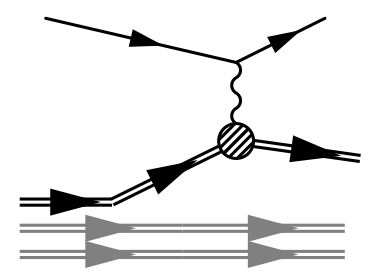

Figure 3-23: Leading-order diagram in the QE electron-proton interaction.

ation. If the incoming (initial-state) or outgoing (final-state) electron interacts with the Coulomb field of a nucleus other than the one it is scattering off, and emits a photon, and the process is called external Bremsstrahlung. If, instead, the electron interacts with the Coulomb field of the nucleus it scatters from, then the process is called internal Bremsstrahlung. The electron can also emit and re-absorb a virtual photon, exchange two photons with the proton, etcetera. Some of these processes are illustrated in Fig. 3-24.
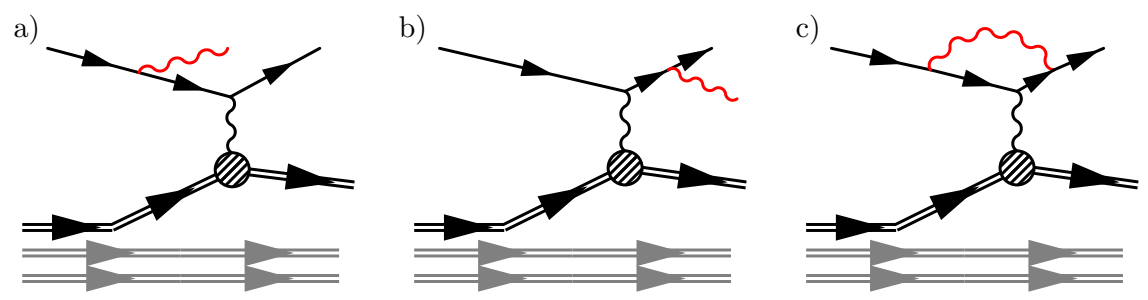

Figure 3-24: Some examples of higher-order electron-proton scattering diagrams: a) initial-state Bremsstrahlung radiation. b) final-state Bremsstrahlung radiation. c) emission and re-absorption of a virtual photon. The radiated photons are represented in red.

These emitted photons go undetected, and the processes are experimentally indistinguishable. The effect from the radiative processes is that, at the vertex, the interaction does not take place at the exact energy predicted from the measurement of the incoming and scattered electron momentum vectors. The proton can also radiate, although the energy loss in this process is suppressed by $m_{e}^{2} / m_{p}^{2}$. In order to extract the Born cross section from the measurement, the contributions from these (and other) diagrams need to be unfolded, and their effect removed.

The radiative correction methodology was first developed by J. Schwinger [122] and later expanded for inclusive $\left(e, e^{\prime}\right)$ experiments by Mo and Tsai [119]. SIMC 
implements radiative effects using the Mo and Tsai formalism generalized for coincidence $\left(e, e^{\prime} p\right)$ cross sections. This formalism is described in detail in [123, 124]. The energy distribution for multi-photon Bremsstrahlung is calculated to all orders using the soft-photon approximation $\left(E_{\gamma} \ll E_{e}, E_{e^{\prime}}, E_{p}\right)$ with some corrections to remove non-physical divergences introduced by this approximation. The angular distribution of these photons uses the "peaking approximation", which assumes the emitted Bremsstrahlung photons are emitted in the directions of the incoming and outgoing electron, and the knocked-out proton.

Fig. 3-25 shows the low- $p_{\text {miss }}{ }^{3}$ He missing-energy spectrum simulated using SIMC with and without radiative effects. The Born spectrum has a visible gap between the 2- and 3-body breakup channels. Radiative effects cause the radiative tail from the 2-body breakup to 'leak' into the 3-body breakup regime, filling this gap and making it experimentally impossible to separate the 2- and 3-body breakup channels on an event-by-event basis. In addition, the $2 \mathrm{bbu}$ radiative tail is larger than the $3 \mathrm{bbu}$ cross section at large $E_{\text {miss }}$.

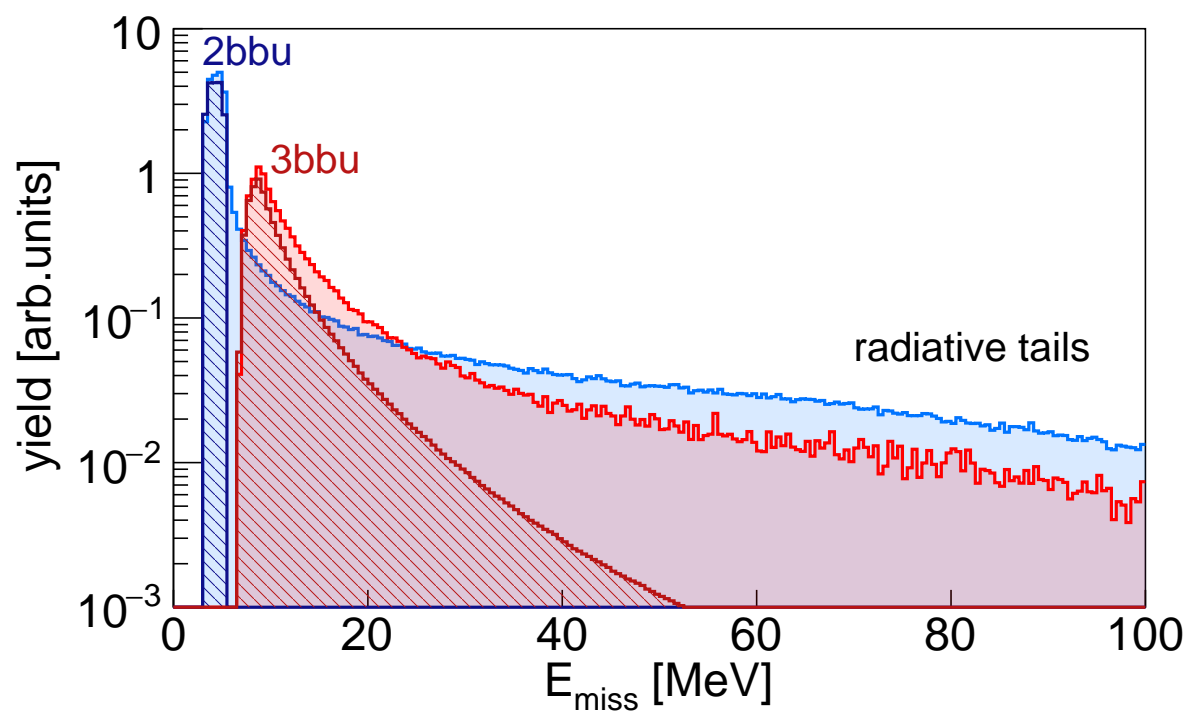

Figure 3-25: Simulated missing-energy spectrum for ${ }^{3} \mathrm{He}$ in the low- $p_{\text {miss }}$ setting with and without radiative effects. The blue and red distributions correspond to the 2- and 3-body breakup channels respectively. The dark-colored curves with diagonal shading correspond to a Born (no radiation) simulation. The light-colored curves with solid filling correspond to a simulation that includes radiative effects calculated following the method described in this section. 
The data was corrected for radiation effects by dividing the measured yield ratio by:

$$
C_{\text {Rad }}\left(p_{\text {miss }}\right)=\frac{R_{\text {Gen.Var }}^{\text {Rad }}\left(p_{\text {miss }}\right)}{R_{\text {Gen.Var }}^{\text {Born }}\left(p_{\text {miss }}\right)},
$$

where $R_{\text {Gen.Var }}^{\text {Rad }}\left(p_{\text {miss }}\right)$ and $R_{\text {Gen.Var }}^{\text {Born }}\left(p_{\text {miss }}\right)$ are the ${ }^{3} \mathrm{He} /{ }^{3} \mathrm{H}$ ratios simulated using SIMC with the $\mathrm{CK}+C C 1$ cross-section model, with and without radiative effects respectively, calculated using generated variables. Fig. 3-27 shows the radiative and other correction factors.

\subsubsection{Bin-migration corrections}

In counting experiments like this one, ensembles of events sampled from an unknown distribution are collected and sorted into bins. The resulting histogram is interpreted as a reproduction of the distribution from which the events came. The variables used to construct these histograms are measured using detectors that have a finite resolution and other detector effects. This can cause events from one bin to be incorrectly assigned to a different one. This bin migration can cause problems if the distribution being measured (e.g. cross sections) is changing rapidly, leading to more events migrating out of a bin than migrating in (or vice versa).

Unlike for data events, we know both the generated variables and the "measured" variables for simulated events. The bin-migration correction was done by dividing the measured yield ratio by:

$$
C_{B M}\left(p_{\text {miss }}\right)=\frac{R_{\text {Rec.Var }}^{\text {Rad }}\left(p_{\text {miss }}\right)}{R_{\text {Gen.Var }}^{\text {Rad }}\left(p_{\text {miss }}\right)},
$$

where $R_{\text {Rec.Var }}^{\text {Rad }}\left(p_{\text {miss }}\right)$ and $R_{\text {Gen.Var }}^{\text {Rad }}\left(p_{\text {miss }}\right)$ are the ${ }^{3} \mathrm{He} /{ }^{3} \mathrm{H}$ ratios evaluated as a function of missing momentum calculated using the reconstructed and generated variables respectively, and including radiative effects. These quantities were obtained using SIMC with the $\mathrm{CK}+C C 1$ cross-section model. The bin-migration effects are small due to the excellent spectrometer resolution, see Fig. 3-27. 


\subsection{5 $E_{m i s s}$-acceptance corrections}

The goal of this analysis is to extract the ratio of ${ }^{3} \mathrm{He} /{ }^{3} \mathrm{H}$ momentum distributions as a function of missing momentum. As stated before (see section 2.2), momentum distributions are obtained by integrating spectral functions over all missing energies: $n\left(p_{\text {miss }}\right)=\int_{0}^{\infty} S\left(p_{\text {miss }}, E_{\text {miss }}\right) d E_{\text {miss }}$. The limited spectrometer momentum and angular acceptances cause a limited $E_{\text {miss }}$ acceptance. For a given $p_{\text {miss }}$ bin, we can only integrate the extracted spectral function over a limited $E_{\text {miss }}$ range. Fig. 3-26 shows an example toy model to illustrate this idea. In it, I generated electron and proton vectors within the 6-dimensional volume corresponding to the HRS acceptance at the high- $p_{\text {miss }}$ kinematical setting, and calculated $p_{\text {miss }}$ and $E_{\text {miss }}$. The finite $E_{\text {miss }}$ acceptance is clearly seen.

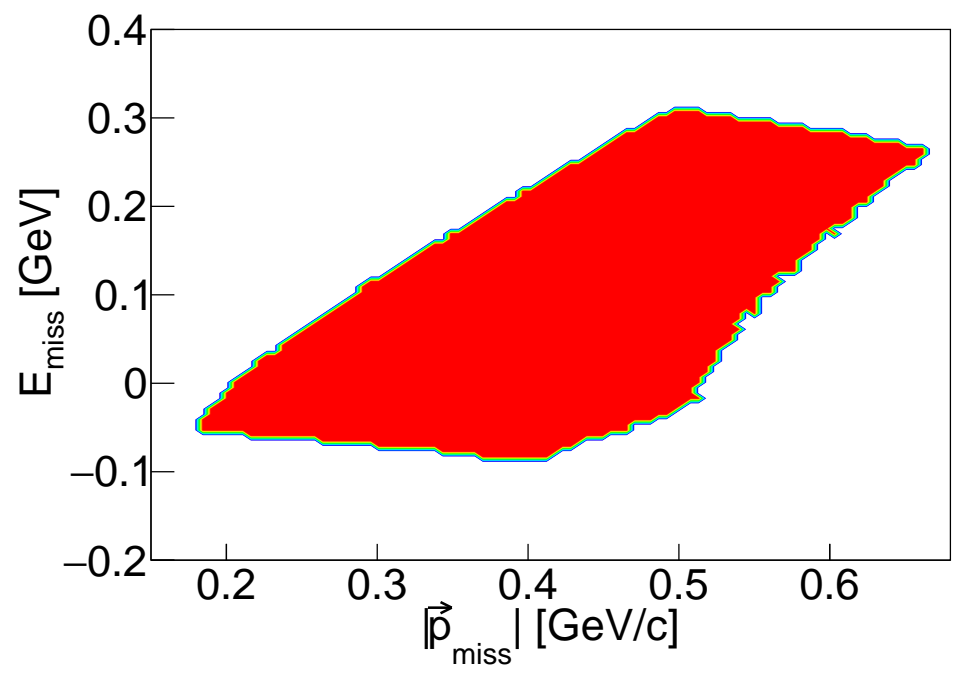

Figure 3-26: Toy model to illustrate the spectrometer limited $E_{\text {miss }}$ acceptance. Electron and proton vectors are generated in the high- $p_{\text {miss }}$ kinematical setting within the spectrometer 6 -dimensional acceptance volume. These generated vectors are used to calculate $p_{\text {miss }}$ and $E_{\text {miss }}$, which we plot here. The red area corresponds to the $p_{\text {miss }}-E_{\text {miss }}$ phase-space available to the spectrometers in this specific kinematical setting.

The $E_{m i s s}$-acceptance correction is achieved by dividing the measured yield ratio by:

$$
C_{E m A c c}\left(p_{m i s s}\right)=\frac{R_{\text {Gen.Var }}^{\text {Born }}\left(p_{m i s s}\right)}{n_{3 \mathrm{He} /{ }^{3} \mathrm{H}}\left(p_{m i s s}\right)},
$$


where:

$$
n_{{ }^{3} \mathrm{He} /{ }^{3} \mathrm{H}}\left(p_{\text {miss }}\right)=\frac{\int_{0}^{\infty} S_{{ }^{3} \mathrm{He}}\left(p_{\text {miss }}, E_{\text {miss }}\right) d E_{\text {miss }}}{\int_{0}^{\infty} S_{{ }^{3} \mathrm{H}}\left(p_{\text {miss }}, E_{\text {miss }}\right) d E_{\text {miss }}},
$$

and:

$$
R_{\text {Gen.Var }}^{\text {Born }}\left(p_{\text {miss }}\right)=\frac{\int_{\Omega_{E_{\text {miss }}}} S_{{ }^{\mathrm{He}}}\left(p_{\text {miss }}, E_{\text {miss }}\right) d E_{\text {miss }}}{\int_{\Omega_{E_{\text {miss }}}} S_{{ }^{\mathrm{H}}}\left(p_{\text {miss }}, E_{\text {miss }}\right) d E_{\text {miss }}} .
$$

Here, $\Omega_{E_{\text {miss }}}$ denotes, for a given $p_{\text {miss }}$ bin, the $E_{\text {miss }}$ range accessible to the spectrometers. The integral from equation 3.39 is calculated by numerically integrating the spectral function used by SIMC to calculate PWIA cross sections. The integral from equation 3.40 is calculated by generating events using the same spectral function (without the inclusion of radiative effects and using generated variables), and putting these events through the SIMC spectrometer acceptance model. The obtained corrections are compared to other corrections in Fig. 3-27.

\subsubsection{Final corrections}

The ${ }^{3} \mathrm{He}\left(e, e^{\prime} p\right) /{ }^{3} \mathrm{H}\left(e, e^{\prime} p\right)$ cross-section ratio as a function of $p_{\text {miss }}$ was extracted by dividing the yield ratio corrected for tritium decay by the total correction:

$$
\frac{\sigma_{3} \mathrm{He}\left(e, e^{\prime} p\right)}{\sigma_{3} \mathrm{H}\left(e, e^{\prime} p\right)}=\frac{1}{C_{\text {total }}} R_{3 \mathrm{He} /{ }^{3} \mathrm{H}}^{\text {corr.yield }},
$$

where the total correction corresponds to the product of the individual corrections described in the previous sections. Some factors from the individual corrections cancel when multiplied together, and the total correction simplifies to:

$$
C_{\text {total }}=C_{B M} \times C_{R a d} \times C_{E m A c c}=\frac{R_{\text {Rec.Var }}^{\text {Rad }}\left(p_{m i s s}\right)}{n_{3 \mathrm{He} /{ }^{3} \mathrm{H}}\left(p_{m i s s}\right)} .
$$

The total corrections are shown in Fig. 3-27, and tabulated in Appendix C Table C.5. The individual correction terms, as well as the total correction, are less than $13.5 \%$. 


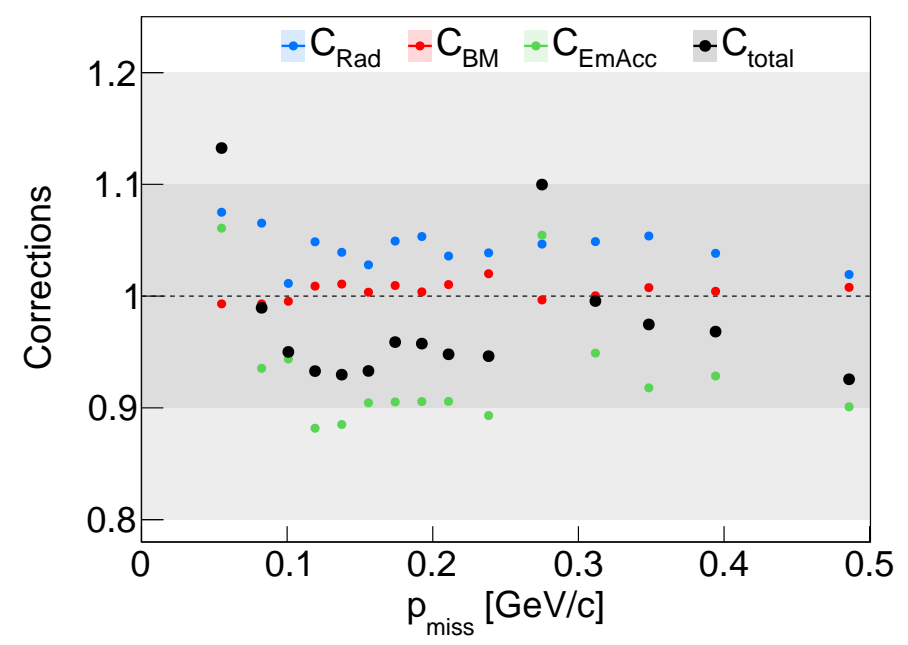

Figure 3-27: Corrections applied to the measured normalized event yield ratio $R_{3}^{\text {corr.yield }}{ }^{3} \mathrm{H}\left(p_{\text {miss }}\right)$ to obtain the ${ }^{3} \mathrm{He}\left(e, e^{\prime} p\right) /{ }^{3} \mathrm{H}\left(e, e^{\prime} p\right)$ cross-section ratio. The blue, red, and green markers correspond to the radiative, bin-migration, and $E_{m i s}$-acceptance corrections respectively. The total correction, which is defined as the product of these corrections, is shown in black. The dark- and light-gray bands correspond to the 10 and $20 \%$ levels respectively.

\subsubsection{Systematic uncertainties}

The point-to-point systematic uncertainties due to the event-selection cuts were determined by repeating the analysis 5000 times. Each time, each event-selection cut was chosen randomly (uniformly) within reasonable limits. See Table 3.7. Each resulting ${ }^{3} \mathrm{He} /{ }^{3} \mathrm{H}$-ratio value for each $p_{\text {miss }}$ bin was histogrammed. The standard deviation of the resulting distribution for each $p_{\text {miss }}$ bin was taken to be the systematic uncertainty from the event-selection cuts in that bin. These values range from $1 \%$ to $8 \%$ and are typically much smaller than the statistical uncertainties.

We assume an additional point-to-point systematic uncertainty in each $p_{\text {miss }}$ bin equal to $20 \%$ of the total correction from section 3.6.6. Appendix C Table C.5 tabulates the correction terms and the resulting cross-section ratios and uncertainties for each $p_{\text {miss }}$ bin.

There is an additional overall normalization uncertainty of $1.8 \%$ coming mainly from the target density uncertainty. Other normalization uncertainties are 1\% or less, as shown in Table 3.8 . 
Table 3.7: Sampling limits for systematic sensitivity study. The $\left|v_{z}\right|, \delta, \theta_{t g}$, and $\phi_{t g}$ limits were used for both LHRS and RHRS reconstructed variables. * was only used in the high- $p_{m i s s}$ kinematical setting.

\begin{tabular}{c|ccc} 
& Units & Lower limit & Upper limit \\
\hline \hline$\left|v_{z}\right|$ & $\mathrm{cm}$ & 8 & 11 \\
$\delta$ & $\%$ & 3.5 & 4.5 \\
$\theta_{t g}$ & $\mathrm{mrad}$ & 50 & 60 \\
$\phi_{t g}$ & $\mathrm{mrad}$ & 25 & 30 \\
$x_{B}^{*}$ & & 1.275 & 1.325 \\
$\theta_{r q}$ & deg & 35 & 40
\end{tabular}

Table 3.8: Overall systematic uncertainties in the extraction of the ${ }^{3} \mathrm{He} /{ }^{3} \mathrm{H}\left(e, e^{\prime} p\right)$ cross-section ratio. All uncertainties are summed in quadrature.

\begin{tabular}{c|c} 
& Overall Uncertainty \\
\hline \hline Target Walls & $\ll 1 \%$ \\
Target Density & $1.5 \%$ \\
Beam-Charge and Stability & $1 \%$ \\
Tritium Decay & $0.18 \%$ \\
\hline Total & $\approx 1.8 \%$
\end{tabular}

\subsubsection{Final results}

Fig. 3-28 and Appendix C Table C.5 show the $p_{\text {miss }}$ dependence of the extracted ${ }^{3} \mathrm{He} /{ }^{3} \mathrm{H}\left(e, e^{\prime} p\right)$ cross-section ratio. In the simplest model, this ratio should be equal to two, the relative number of protons in ${ }^{3} \mathrm{He}$ and ${ }^{3} \mathrm{H}$. However, at large $p_{\text {miss }}$ the ratio should be equal to one, the relative number of $n p$-SRC pairs in ${ }^{3} \mathrm{He}$ and ${ }^{3} \mathrm{H}$. These SRC pairs will shift equal amounts of cross-section strength from low $p_{\text {miss }}$ to high $p_{\text {miss }}$ in both nuclei, increasing the ${ }^{3} \mathrm{He}$ to ${ }^{3} \mathrm{H}$ ratio at low $p_{\text {miss }}$ to more than two. The measured ratio follows this simple model of a transition from independent nucleons at the lowest $p_{\text {miss }}$ to $n p$-SRC pairs at higher $p_{\text {miss }}$, decreasing from almost three at low $p_{\text {miss }}$ towards about 1.5 at $p_{\text {miss }}=250 \mathrm{MeV} / \mathrm{c}$. At larger $p_{\text {miss }}$ the measured ratio is approximately flat, with a possible rise at the largest $p_{m i s s}$.

Since we chose kinematics in which reaction mechanisms were expected to be small, the resulting cross-section ratios should be sensitive to the ratio of momentum distributions. We therefore compare in Fig. 3-28 the measured cross-section ratios with the 


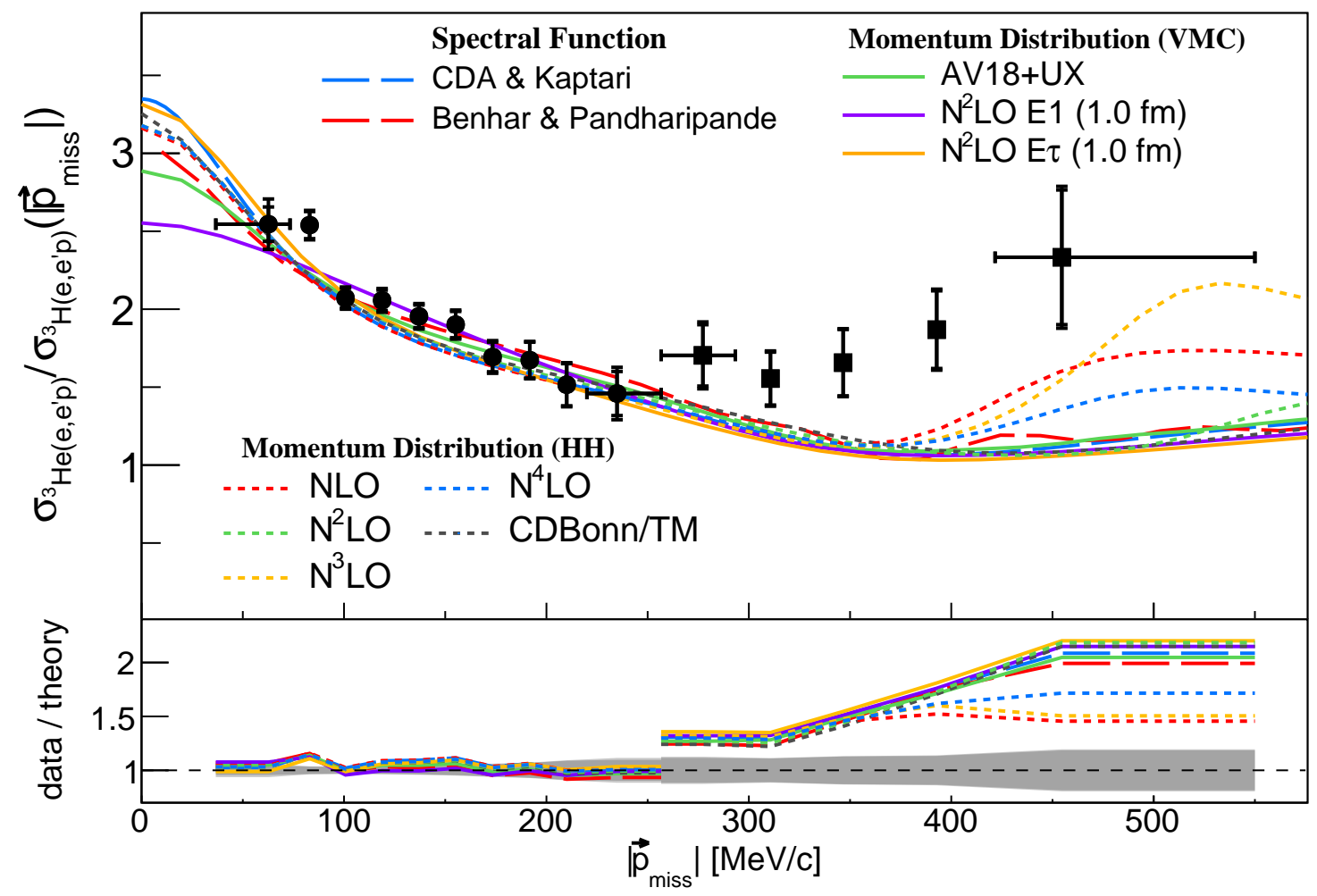

Figure 3-28: ${ }^{3} \mathrm{He} /{ }^{3} \mathrm{H}$ cross-section ratio as a function of $p_{\text {miss }}$ for the low- $p_{\text {miss }}(\bullet)$ and high- $p_{\text {miss }}(\mathbb{\square})$ kinematical settings compared with different momentum-distribution ratios. The solid lines correspond to momentum distributions calculated using the VMC technique [66, 73]. The long-dashed lines (--) correspond to spectral function calculations integrated over their energy dependence [69, 125]. The dashed lines (---) corresponds to momentum distributions calculated using the $\mathrm{HH}$ technique [126, 127]. See text for details. 
ratio of single-nucleon momentum distributions. The momentum distribution calculations are obtained using either the variational Monte Carlo (VMC) technique with local interactions [66, 73] or the Hyperspherical Harmonics (HH) method [126, 127] with non-local interactions. While momentum distributions calculated with local chiral-interactions depend strongly on the cutoff parameter, these effects mostly cancel in the ratio of the momentum distributions. In the case of the non-local chiral potential models, the calculations show significant order dependence. Additionally, the momentum-distribution ratio calculated by integrating the spectral functions from Ciofi degli Atti and Kaptari [69] and Benhar [125] over missing energy are shown.

We found that all the momentum-distribution ratios shown agree with the measurement for $p_{\text {miss }} \lesssim 250 \mathrm{MeV} / c$. On the other hand, for $p_{\text {miss }} \gtrsim 250 \mathrm{MeV} / c$ there is an unexpected $20-50 \%$ discrepancy which had already been seen in Fig. 3-22. Some of the possible explanations for this discrepancy are:

- Breakdown of the factorized approach from equation 2.5

- Additional FSI effects

- Single-charge exchange (SCX)

- Relativistic effects.

If all these effects are proven to be negligible, this disagreement could point to issues with the underlying $N N$ interaction at short distances.

\section{Breakdown of the factorized approach}

To test the factorization approximation of equation 2.5, we compared the SIMC factorized $\mathrm{CK}+C C 1$ model with the unfactorized calculation by the Cracow group (see section 2.2.3). The level of agreement between the ${ }^{3} \mathrm{He} /{ }^{3} \mathrm{H}$ ratios extracted with either model was in the order of $5 \%$. That is, a breakdown of the factorization approximation is not enough to explain the high- $p_{m i s s}$ discrepancy. 


\section{Additional FSI effects}

While the experiment kinematics were specifically selected to minimize effects such as FSI, and these effects should further cancel in the ratio of cross sections, we studied the size of residual FSI effects using the cross-section model of M. Sargsian (see section 2.2.3). This model includes the effects of the PWIA diagram, and the diagram corresponding to single-rescattering between the knocked-out proton and either of the other two nucleons in the 3-body breakup channel calculated in the generalized Eikonal approximation [79, 80]. For each $p_{\text {miss }}$ bin in the high- $p_{\text {miss }}$ kinematical setting, we calculated the ${ }^{3} \mathrm{He}$ and ${ }^{3} \mathrm{H}$, PWIA and FSI cross sections integrated over the experimental $E_{m i s s}$ acceptance, and formed the double ratios:

$$
R_{\mathrm{FSI}} / R_{\mathrm{PWIA}}=\frac{\sigma_{\mathrm{FSI}} /\left.\sigma_{\mathrm{PWIA}}\right|_{{ } \mathrm{He}}}{\sigma_{\mathrm{FSI}} /\left.\sigma_{\mathrm{PWIA}}\right|_{{ } \mathrm{H}}} .
$$

Fig. 3-29 shows this quantity as a function of missing momentum for the high- $p_{\text {miss }}$ kinematical setting. The single-rescattering FSI effect is at most 5\%, and therefore cannot explain the high- $p_{\text {miss }}$ discrepancy. We did not correct the data for this effect.

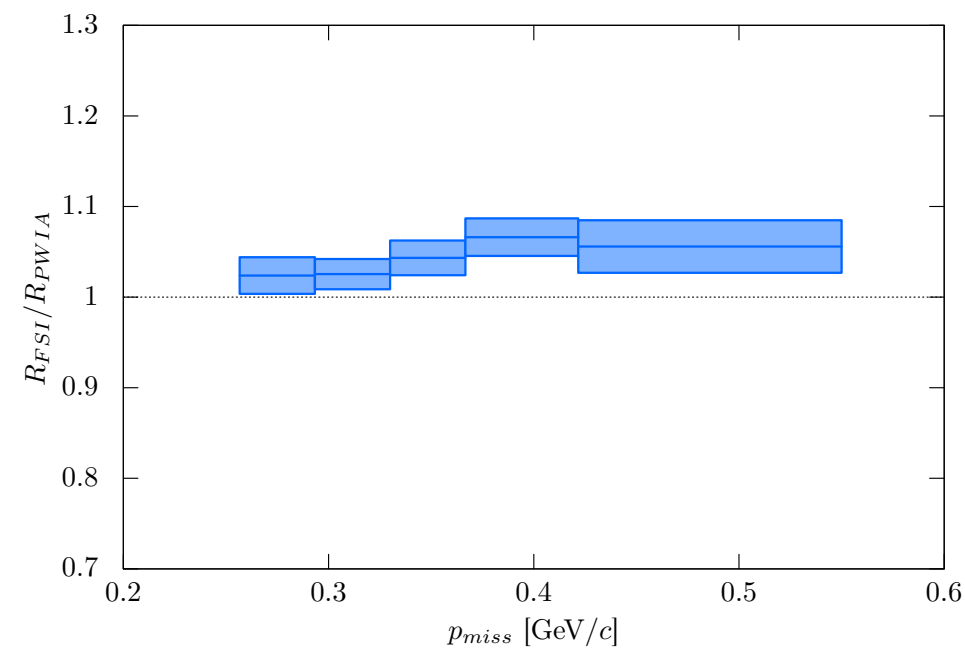

Figure 3-29: Effect of single-rescattering FSI in the ${ }^{3} \mathrm{He} /{ }^{3} \mathrm{H}$ ratio as a function of missing momentum for the high- $p_{\text {miss }}$ kinematical setting. 


\section{Remaining effects}

Another possible explanation for the high- $p_{\text {miss }}$ discrepancy could be single-charge exchange (SCX) in which the struck proton from an $\left(e, e^{\prime} p\right)$ event rescatters at almost $180^{\circ}$ from a neutron, and the latter is ejected from the nucleus ( $p n$-SCX) or vice-versa, the struck neutron from an $\left(e, e^{\prime} n\right)$ event rescatters at almost $180^{\circ}$ from a proton, and the latter is ejected from the nucleus (np-SCX). $p n$-SCX and $n p$-SCX events will respectively decrease and increase the overall number of $\left(e, e^{\prime} p\right)$ events. While these two effects typically balance each other out to some extent, at high- $p_{m i s s}$ and in $A=3$ nuclei this may not be the case. Due to $n p$ dominance, in ${ }^{3} \mathrm{He}$ the uncorrelated nucleon will most likely be a proton, while in ${ }^{3} \mathrm{H}$ it will be a neutron. Thus, if in ${ }^{3} \mathrm{He}$ $n p$-SCX is more likely than $p n$-SCX, then the net effect would be an increase in the $\left(e, e^{\prime} p\right)$ cross section. Oppositely, if in ${ }^{3} \mathrm{H} p n$-SCX is more likely than $n p$-SCX, then the net effect would be a decrease in the $\left(e, e^{\prime} p\right)$ cross section. Additionally, if the SCX process happens at $<180^{\circ}$, then events at small $p_{\text {miss }}$ will be shifted to larger $p_{m i s s}$ states, which will amplify the SCX effects at high- $p_{m i s s}$. This could increase the ${ }^{3} \mathrm{He} /{ }^{3} \mathrm{H}$ ratio and explain the high- $p_{\text {miss }}$ discrepancy. At the time of this analysis, $A=3$ SCX calculations are not available.

Although single-rescattering FSI effects calculated in the generalized Eikonal approximation are small, more complete calculations including two- and three-body interaction operators [128] are needed to fully assess the FSI contribution to the ratio. Furthermore, fully relativistic calculations are needed to study longitudinal-transverse interference effects [129, 130, 131, 132].

At this stage, more theoretical input is needed to determine whether the high$p_{\text {miss }}$ discrepancy is due to unconstrained reaction mechanisms or to issues with the underlying $N N$ interaction at short distances. In the experimental front, we can

determine whether this discrepancy is caused by ${ }^{3} \mathrm{He}\left(e, e^{\prime} p\right)$ events, ${ }^{3} \mathrm{H}\left(e, e^{\prime} p\right)$ events, or both. In order to address this question, we extracted absolute cross sections. 


\section{$3.7 \quad{ }^{3} \mathbf{H e},{ }^{3} \mathbf{H}\left(e, e^{\prime} p\right) \quad$ absolute cross sections}

For each measured nucleus we binned the data in bins of $p_{\text {miss }}$ and $E_{\text {miss }}$ and calculated the raw cross section as:

$$
\left.\left.\sigma\left(p_{\text {miss }}, E_{\text {miss }}\right)\right|_{\text {Raw }} \equiv \frac{d^{6} \sigma\left(p_{\text {miss }}, E_{\text {miss }}\right)}{d E_{e^{\prime}} d \Omega_{e^{\prime}} d E_{p} d \Omega_{p}}\right|_{\text {Raw }}=\frac{N\left(p_{\text {miss }}, E_{\text {miss }}\right)}{C\left(\frac{\rho}{A} \cdot b\right) \cdot t_{\text {live }} \cdot V_{B}\left(p_{\text {miss }}, E_{\text {miss }}\right)}
$$

where $N\left(p_{\text {miss }}, E_{\text {miss }}\right)$ is the number of counts measured in each $\left(p_{\text {miss }}, E_{\text {miss }}\right)$ bin, and $V_{B}\left(p_{m i s s}, E_{m i s s}\right)$ is a factor determined from SIMC simulations that accounts for the spectrometer acceptance and detected phase-space volume of each $p_{\text {miss }}$ and $E_{\text {miss }}$ bin (see section 3.7.1).

We then corrected the raw cross sections for radiative and bin-migration effects, integrated over $E_{m i s s}$, and then bin-centered (see section 3.7.4). Finally, the ${ }^{3} \mathrm{H}$ events were corrected for radioactive decay.

The $E_{\text {miss }}$ integration was defined as:

$$
\sigma\left(p_{m i s s}\right) \equiv \sum_{j} \sigma\left(p_{m i s s}, E_{m i s s}^{j}\right) \Delta E_{m i s s}^{j}
$$

where $\Delta E_{m i s s}^{j}$ corresponds to the bin width. The $E_{\text {miss }}$ integration limits for each $p_{\text {miss }}$ bin correspond to the blue area shown in Fig. $3-30$. The lower $E_{\text {miss }}$ limit corresponds to $8 \mathrm{MeV}$ (i.e. above the ${ }^{3} \mathrm{He} 2$-body breakup peak) to only include the ${ }^{3} \mathrm{He} 3$-body breakup channel and allow for a more consistent comparison with ${ }^{3} \mathrm{H}$ (in which only the 3-body breakup channel is present. See section 2.2.1. At low- $p_{\text {miss }}$, we excluded bins with $E_{\text {miss }}>50 \mathrm{MeV}$ due to the size of the radiative corrections (see section 3.7.2). We also excluded bins with zero measured events, and bins at the edge of the spectrometer acceptance, where SIMC simulations are less reliable. We eliminated unreliable bins by simulating events with a constant cross section, analyzing the simulated events the same way as the data, and excluding bins for which the cross section extracted from the reconstructed simulated events differed from the input cross section by more than $5 \%$. 


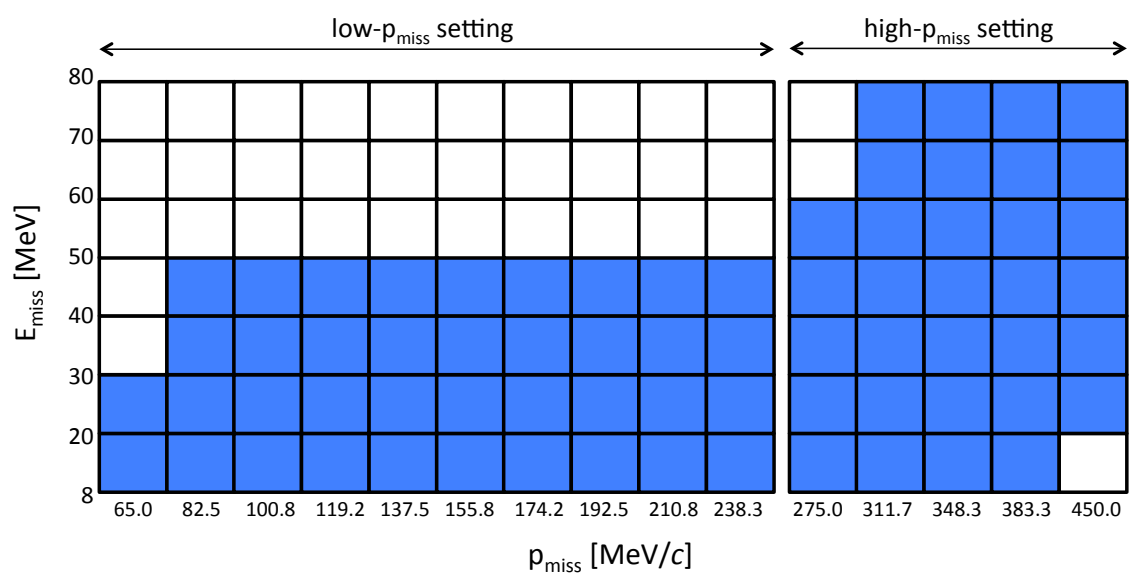

Figure 3-30: $E_{\text {miss }}$ integration limits for each $p_{\text {miss }}$ bin. The included bins are shown in blue. Bins excluded are those with no measured events, large radiative corrections, or bins that are near the spectrometer acceptance edges. See text for details.

\subsubsection{Phase-space and acceptance correction factors}

The acceptance-corrected phase-space factors are determined from simulation. We generated $N_{\text {tot }}$ events uniformly sampling in the ranges $\Delta E_{e^{\prime}}, \Delta \Omega_{e^{\prime}}, \Delta E_{p}$, and $\Delta \Omega_{p}$ (each defined in ranges larger than the spectrometer acceptance). Out of this total, we determined the events generated in each $\left(p_{\text {miss }}, E_{\text {miss }}\right)$ bin, $N\left(p_{\text {miss }}, E_{\text {miss }}\right)$. Then, the phase-space factor is:

$$
\Phi\left(p_{m i s s}, E_{m i s s}\right)=\frac{N\left(p_{m i s s}, E_{m i s s}\right)}{N_{\text {tot }}} \times\left(\Delta E_{e^{\prime}} \Delta \Omega_{e^{\prime}} \Delta E_{p} \Delta \Omega_{p}\right)
$$

From the $N\left(p_{\text {miss }}, E_{\text {miss }}\right)$ events generated in each $\left(p_{m i s s}, E_{m i s s}\right)$ bin, we determined the number of events accepted in each bin, $N_{\text {acc }}\left(p_{\text {miss }}, E_{m i s s}\right)$. Then, the acceptance factor is:

$$
\operatorname{ACC}\left(p_{m i s s}, E_{m i s s}\right)=\frac{N_{\mathrm{acc}}\left(p_{m i s s}, E_{m i s s}\right)}{N\left(p_{m i s s}, E_{m i s s}\right)}
$$

Since the factors $N\left(p_{\text {miss }}, E_{\text {miss }}\right)$ cancel out in the $\Phi \times \mathrm{ACC}$ product, this correction factor: 


$$
\begin{aligned}
V_{B}\left(p_{\text {miss }}, E_{\text {miss }}\right) & \equiv \Phi\left(p_{\text {miss }}, E_{\text {miss }}\right) \times \operatorname{ACC}\left(p_{\text {miss }}, E_{\text {miss }}\right) \\
& =\frac{N_{\text {acc }}\left(p_{\text {miss }}, E_{\text {miss }}\right)}{N_{\text {tot }}} \times\left(\Delta E_{e^{\prime}} \Delta \Omega_{e^{\prime}} \Delta E_{p} \Delta \Omega_{p}\right)
\end{aligned}
$$

was determined directly. See Table 3.9 .

Table 3.9: $\quad V_{B}$ (acceptance-corrected phase-space) correction values in each $\left(p_{\text {miss }}, E_{\text {miss }}\right)$ bin, in units of $\mathrm{MeV}^{2} \mathrm{sr}^{2}$.

\begin{tabular}{c|c|c|c|c|c|c|c} 
& \multicolumn{7}{|c}{$E_{\text {miss }}[\mathrm{MeV}]$} \\
$p_{\text {miss }}[\mathrm{MeV} / c]$ & $8-20$ & $20-30$ & $30-40$ & $40-50$ & $50-60$ & $60-70$ & $70-80$ \\
\hline \hline $36.7-73.3$ & $1.039 \mathrm{e}-04$ & $4.393 \mathrm{e}-05$ & - & - & - & - & - \\
$73.3-91.7$ & $2.942 \mathrm{e}-04$ & $1.850 \mathrm{e}-04$ & $1.174 \mathrm{e}-04$ & $6.342 \mathrm{e}-05$ & - & - & - \\
$91.7-110.0$ & $5.799 \mathrm{e}-04$ & $4.295 \mathrm{e}-04$ & $3.554 \mathrm{e}-04$ & $2.584 \mathrm{e}-04$ & $1.629 \mathrm{e}-04$ & $8.032 \mathrm{e}-05$ & $2.859 \mathrm{e}-05$ \\
$110.0-128.3$ & $8.562 \mathrm{e}-04$ & $7.015 \mathrm{e}-04$ & $6.665 \mathrm{e}-04$ & $5.735-04$ & $4.579 \mathrm{e}-04$ & $3.072 \mathrm{e}-04$ & $1.753 \mathrm{e}-04$ \\
$128.3-146.7$ & $1.075 \mathrm{e}-03$ & $9.243 \mathrm{e}-04$ & $9.072 \mathrm{e}-04$ & $8.908 \mathrm{e}-04$ & $7.979 \mathrm{e}-04$ & $6.562 \mathrm{e}-04$ & $4.834 \mathrm{e}-04$ \\
$146.7-165.0$ & $1.262 \mathrm{e}-03$ & $1.075 \mathrm{e}-03$ & $1.086 \mathrm{e}-03$ & $1.092 \mathrm{e}-03$ & $1.043 \mathrm{e}-03$ & $9.231 \mathrm{e}-04$ & $7.830 \mathrm{e}-04$ \\
$165.0-183.3$ & $1.415 \mathrm{e}-03$ & $1.207 \mathrm{e}-03$ & $1.236 \mathrm{e}-03$ & $1.242 \mathrm{e}-03$ & $1.206 \mathrm{e}-03$ & $1.075 \mathrm{e}-03$ & $9.520 \mathrm{e}-04$ \\
$183.3-201.7$ & $1.563 \mathrm{e}-03$ & $1.329 \mathrm{e}-03$ & $1.373 \mathrm{e}-03$ & $1.378 \mathrm{e}-03$ & $1.322 \mathrm{e}-03$ & $1.192 \mathrm{e}-03$ & $1.066 \mathrm{e}-03$ \\
$201.7-220.0$ & $1.636 \mathrm{e}-03$ & $1.420 \mathrm{e}-03$ & $1.465 \mathrm{e}-03$ & $1.497 \mathrm{e}-03$ & $1.439 \mathrm{e}-03$ & $1.287 \mathrm{e}-03$ & $1.151 \mathrm{e}-03$ \\
$220.0-256.7$ & $2.918 \mathrm{e}-03$ & $2.577 \mathrm{e}-03$ & $2.728 \mathrm{e}-03$ & $2.829 \mathrm{e}-03$ & $2.839 \mathrm{e}-03$ & $2.670 \mathrm{e}-03$ & $2.428 \mathrm{e}-03$ \\
\hline $256.7-293.3$ & $8.967 \mathrm{e}-04$ & $6.100 \mathrm{e}-04$ & $4.480 \mathrm{e}-04$ & $2.993 \mathrm{e}-04$ & $1.530 \mathrm{e}-04$ & - & - \\
$293.3-330.0$ & $1.977 \mathrm{e}-03$ & $1.764 \mathrm{e}-03$ & $1.718 \mathrm{e}-03$ & $1.567 \mathrm{e}-03$ & $1.321 \mathrm{e}-03$ & $9.754 \mathrm{e}-04$ & $5.406 \mathrm{e}-04$ \\
$330.0-366.7$ & $2.635 \mathrm{e}-03$ & $2.481 \mathrm{e}-03$ & $2.525 \mathrm{e}-03$ & $2.516 \mathrm{e}-03$ & $2.453 \mathrm{e}-03$ & $2.355 \mathrm{e}-03$ & $2.111 \mathrm{e}-03$ \\
$366.7-400.0$ & $2.763 \mathrm{e}-03$ & $2.518 \mathrm{e}-03$ & $2.569 \mathrm{e}-03$ & $2.585 \mathrm{e}-03$ & $2.618 \mathrm{e}-03$ & $2.633 \mathrm{e}-03$ & $2.610 \mathrm{e}-03$ \\
$400.0-500.0$ & - & $4.878 \mathrm{e}-03$ & $5.302 \mathrm{e}-03$ & $5.734 \mathrm{e}-03$ & $6.135 \mathrm{e}-03$ & $6.568 \mathrm{e}-03$ & $6.982 \mathrm{e}-03$
\end{tabular}

\subsubsection{Radiative corrections}

The radiative corrections were determined as:

$$
C_{\text {Rad }}\left(p_{\text {miss }}, E_{\text {miss }}\right)=\frac{Y_{\text {Gen.Var }}^{\text {Rad }}\left(p_{\text {miss }}, E_{\text {miss }}\right)}{Y_{\text {Gen.Var }}^{\text {Born }}\left(p_{\text {miss }}, E_{\text {miss }}\right)}
$$

where $Y$ refers to the normalized yield. Since in this case, unlike in the ratio analysis, only the ${ }^{3} \mathrm{He} 3$-body breakup channel was included, the 2-body breakup channel contribution was studied more carefully. SIMC simulations were run separately for each channel (including radiative effects) and the simulated data was analyzed following the same event-selection criteria used for the experimental data. The resulting normalized yield as a function of $E_{\text {miss }}$ can be written as: 


$$
Y_{{ }_{3}^{\mathrm{He}}}^{\mathrm{Rad}}\left(E_{\text {miss }}\right)=A\left(Y_{2 b b u}^{\mathrm{Rad}}\left(E_{m i s s}\right)+B \cdot Y_{3 b b u}^{\text {Rad }}\left(E_{m i s s}\right)\right)
$$

We simultaneously fitted the $2 \mathrm{bbu}$ and $3 \mathrm{bbu}$ simulated spectra to the experimental yield, and the coefficients $A$ and $B$ were determined. Fig. 3-31 shows the resulting $E_{\text {miss }}$ distributions. We binned the $2 \mathrm{bbu}$ peak in a single bin from $0-8 \mathrm{MeV}$ to avoid fitting issues coming from its resolution and position. The extracted coefficients $A$ and $B$ are summarized in Table 3.10 .
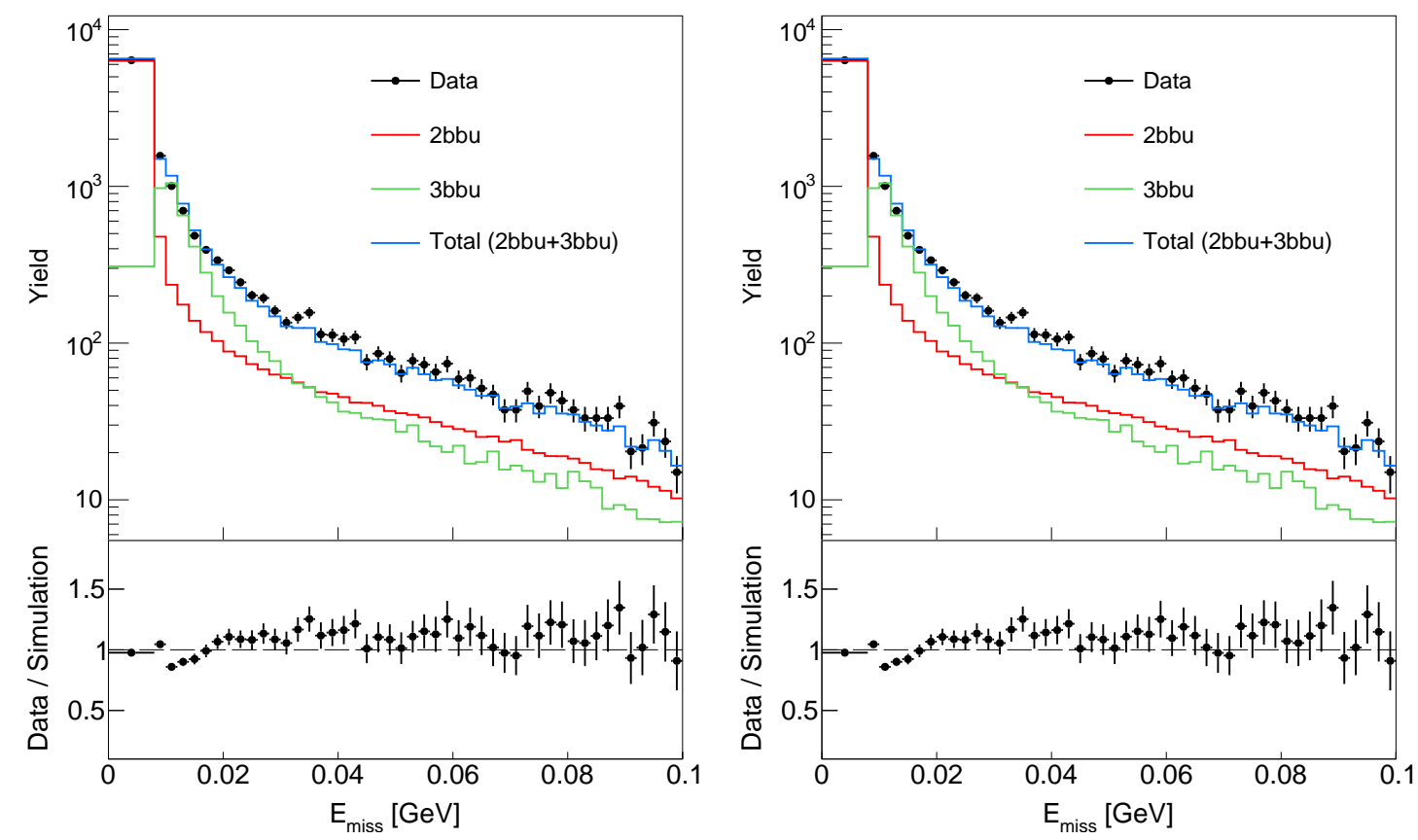

Figure 3-31: Relative 2- and 3-body breakup contributions to the measured yield as a function of $E_{m i s s}$ for the low- $p_{m i s s}$ (left) and high- $p_{m i s s}$ (right) kinematical settings. The black markers correspond to the normalized measured yield. The blue line corresponds to the total simulated yield scaled by $A$ and $B$ (see text for details). The red and green lines correspond to the $2 \mathrm{bbu}$ and $3 \mathrm{bbu}$ contributions respectively. The bottom panels show the ratio of the measured to the total simulated spectra.

Table 3.10: Two- and three-body breakup coefficients for the low- and high- $p_{\text {miss }}$ kinematical settings.

\begin{tabular}{c|c|c} 
& low- $p_{\text {miss }}$ kinematics & high- $p_{\text {miss }}$ kinematics \\
\hline \hline$A$ & $0.570 \pm 0.010$ & $0.780 \pm 0.095$ \\
$B$ & $1.263 \pm 0.039$ & $1.068 \pm 0.147$
\end{tabular}


The size of the radiative correction depends on the $E_{\text {miss }}$-integration range, as shown in Fig. 3-32. This is due to the fact that, at large $E_{\text {miss }}$, the 3 bbu cross section decreases faster than the radiative tail from the 2 bbu channel (see Fig. 3-31). To reduce the size of the radiative correction factors, we integrated over $E_{\text {miss }}$ up to 50 and $80 \mathrm{MeV}$ in the low- and high- $p_{\text {miss }}$ kinematical settings respectively. The size of the final radiative correction is compared to other corrections in Fig. 3-35 and tabulated in Appendix C Tables C.6 and C.7.
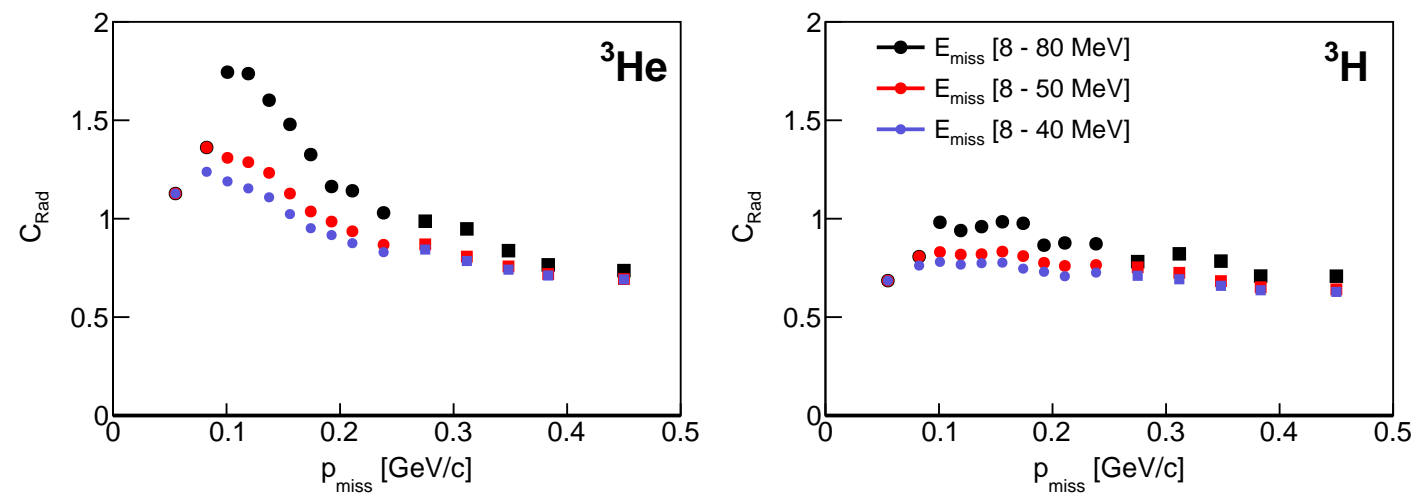

Figure 3-32: Radiative correction factors for the ${ }^{3} \mathrm{He}$ (left) and ${ }^{3} \mathrm{H}$ (right) absolute cross sections integrated over $E_{\text {miss }}$. The different colors correspond to different ranges in the $E_{\text {miss }}$ integration.

\subsubsection{Bin-migration corrections}

The bin-migration corrections were determined as:

$$
C_{B M}\left(p_{m i s s}, E_{m i s s}\right)=\frac{Y_{\text {Rec.Var }}^{\text {Rad }}\left(p_{\text {miss }}, E_{\text {miss }}\right)}{Y_{\text {Gen.Var }}^{\text {Rad }}\left(p_{\text {miss }}, E_{\text {miss }}\right)},
$$

where $Y$ is the normalized yield. For ${ }^{3} \mathrm{He}$, the contribution from $2 \mathrm{bbu}$ and $3 \mathrm{bbu}$ is included following the method described at the end of the previous section. The size of the bin-migration correction is compared to other corrections in Fig. 3-35. The values can be found in Appendix C Tables C.6 and C.7. The bin-migration effects are small due to the excellent spectrometer resolution. 


\subsubsection{Bin-centering corrections}

We applied a bin-centering correction to translate the measured cross section to the center of the $p_{\text {miss }}$ bin to make comparison to future theoretical calculations easier. The bin-centering correction was applied by dividing the cross section (corrected for radiative and bin-migration effects, and integrated over missing energy) by:

$$
C_{B C}\left(p_{\text {miss }}\right)=\frac{\sigma_{\text {integrated }}\left(p_{\text {miss }}\right)}{\sigma_{\text {point }}\left(p_{\text {miss }}\right)} .
$$

Here, $\sigma_{\text {integrated }}\left(p_{\text {miss }}\right)$ corresponds to the acceptance-integrated cross section in each $p_{\text {miss }}$ bin extracted from a SIMC simulation with a given theory model, and integrated over $E_{\text {miss }}$ (i.e. SIMC phase-space events are weighted with a cross-section model, and the simulated data is analyzed following the same procedure used to extract the cross section from the measured data). $\sigma_{\text {point }}\left(p_{\text {miss }}\right)$ corresponds to the cross section calculated using the same theory model in a single kinematical point within that $p_{\text {miss }}$ bin, also integrated over $E_{\text {miss }}$. The kinematical point in which $\sigma_{\text {point }}$ is evaluated corresponds to the central values of $p_{\text {miss }}$ and $E_{\text {miss }}$ and the average values of $x_{B}$ and $Q^{2}$ in that bin, and the electron and proton out-of-plane angles $\phi_{e^{\prime}}=0$ and $\phi_{p}=180^{\circ}$ respectively.

The bin-centering correction factors were determined using both the $\mathrm{CK}+C C 1$ and Cracow models. The total correction was defined as their average, and the uncertainty as their difference (divided by $\sqrt{12}$ ). In the $p_{\text {miss }}$ bins in which the extracted fractional uncertainty was $<2 \%$, we fixed it at $2 \%$. The bin-centering correction factors from either model, as well as the total bin-centering correction factor and its uncertainty are shown in Figs. 3-33 and 3-34 for the low- and high- $p_{\text {miss }}$ kinematical settings respectively.

The integrated-cross-section values used to determine $\sigma_{\text {integrated }}\left(p_{\text {miss }}\right)$ from different models are summarized in Appendix C Tables C.1 and C.2 for the low- and high- $p_{\text {miss }}$ kinematical settings respectively. The point-cross-section values (and kine-

matical points in which they are calculated) used to determine $\sigma_{\text {point }}\left(p_{\text {miss }}\right)$ from different models are summarized in Appendix C Tables C.3 and C.4 for the low- and 

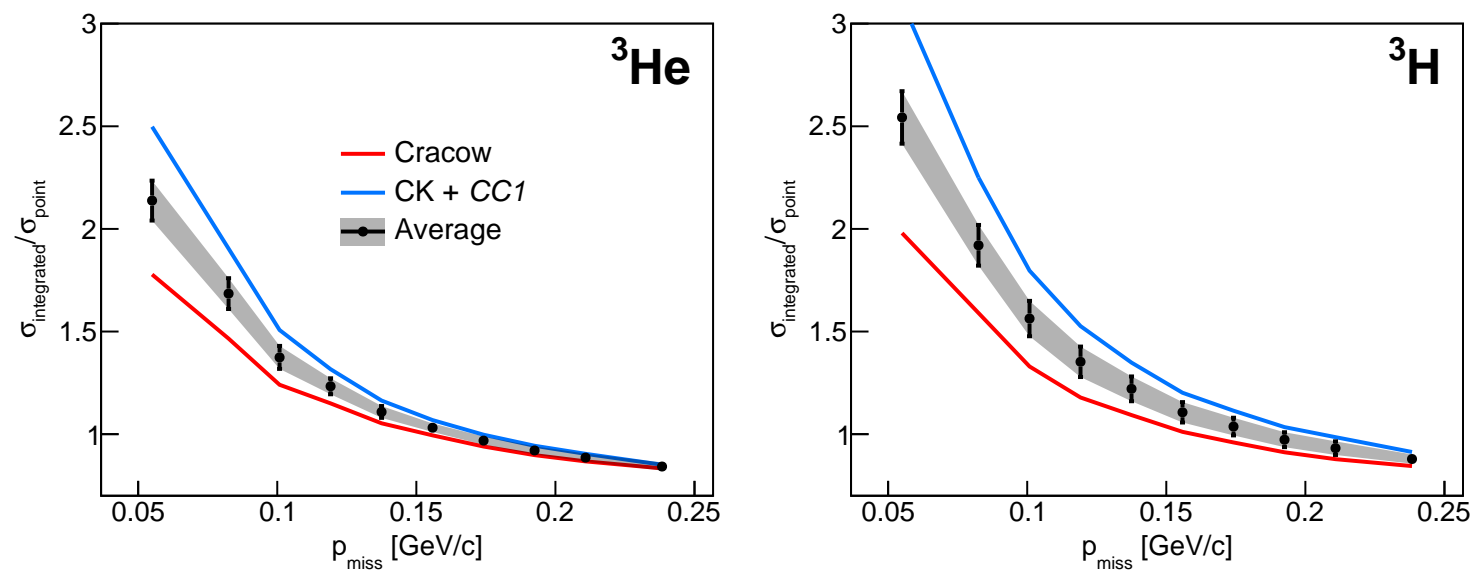

Figure 3-33: Bin-centering correction factors for the low- $p_{\text {miss }}$ kinematical setting for ${ }^{3} \mathrm{He}$ (left) and ${ }^{3} \mathrm{H}$ (right). The red and blue lines correspond to bin-centering correction factors determined with the Cracow and $\mathrm{CK}+C C 1$ models respectively. The total correction is shown as black markers and a gray band.
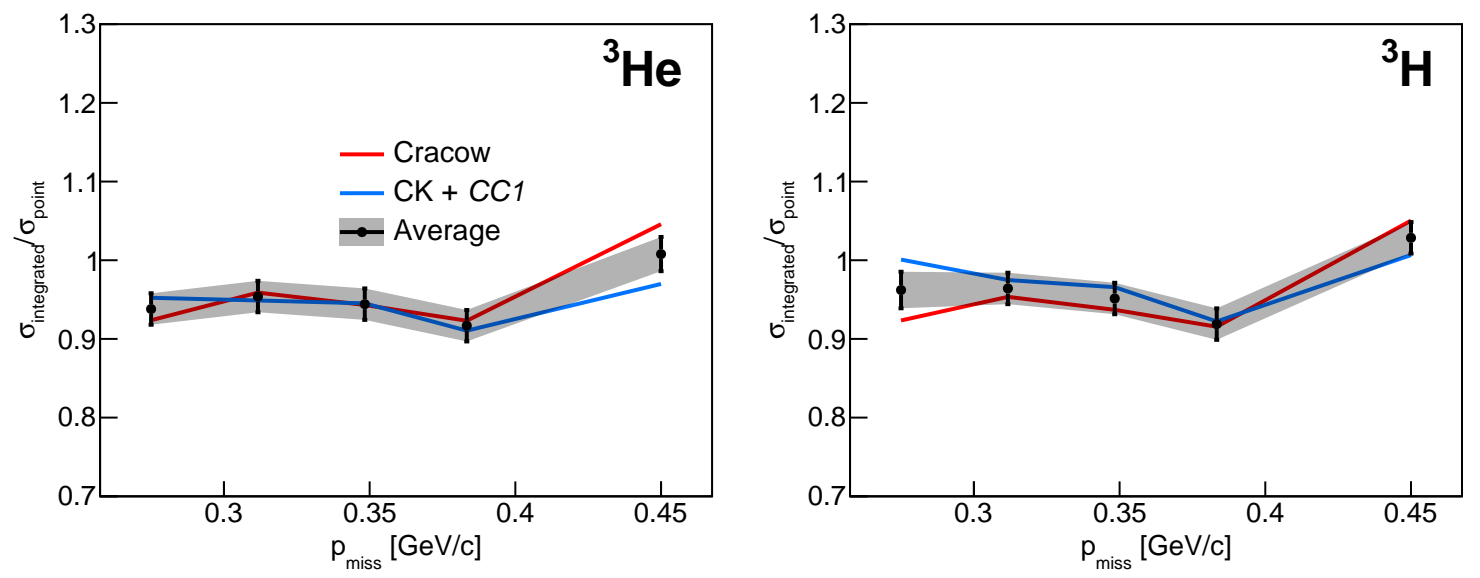

Figure 3-34: Same as Fig. 3-33 for the high- $p_{\text {miss }}$ kinematical setting. 
high- $p_{m i s s}$ kinematical settings respectively.

For completeness, we extracted the acceptance-integrated Sargsian-FSI cross section (which was not used in the determination of bin-centering correction factors) in the high- $p_{m i s s}$ kinematical setting and checked that:

$$
\frac{\sigma_{\text {integrated }}^{\text {Sargsian-FSI }}\left(p_{\text {miss }}\right)}{\sigma_{\text {point }}^{\text {Sargsian-FSI }}\left(p_{\text {miss }}\right)} \approx C_{B C}\left(p_{\text {miss }}\right),
$$

further verifying that using this model for bin-centering corrections does not result in significantly different correction factors.

Future models can be compared to the results from our measurement by calculating point cross sections in the kinematical points from Appendix C Tables C.3 and C.4 and integrating these values over $E_{\text {miss }}$ following equation 3.45 .

\subsection{5 ${ }^{3}$ H-decay correction}

The tritium absolute cross section was corrected for decay by dividing by:

$$
C_{D C} \equiv \frac{\sigma_{{ }^{\mathrm{H}}}^{\text {mixed }}}{\sigma^{\sigma_{\mathrm{H}}}}=\frac{1-\epsilon}{1-\epsilon \cdot R_{{ }^{\mathrm{He}} /{ }^{3} \mathrm{H}}^{\text {measured }}} .
$$

We used the experimental cross-section ratio $R_{3 \mathrm{He} /{ }^{3} \mathrm{H}}^{\text {mear }} \mathrm{H}$ to calculate $C_{D C}$. Error propagation was used to determined the uncertainty of $C_{D C}$ as:

$$
\delta C_{D C}=\frac{\epsilon(1-\epsilon)}{\left(1-\epsilon \cdot R_{3^{3} \mathrm{He} /{ }^{3} \mathrm{H}}^{\text {measured }}\right)^{2}} \delta\left(R^{\text {measured }}\right) .
$$

$\delta\left(R_{3^{3} \mathrm{He} / 3 \mathrm{H}}^{\text {meased }}\right)$ only includes statistical uncertainties to avoid double counting the systematic uncertainties. This correction is compared to other corrections in Fig. 3-35. The values can be found in Appendix C Table C.7.

\subsubsection{Final corrections}

We obtained the absolute cross sections as follows. First, we corrected the raw absolute cross section from equation 3.44 for radiative and bin-migration effects, then 
integrated it over missing energy, and then corrected it for bin-centering. Finally, we corrected the tritium cross section for decay. This process is qualitatively described as:

$$
\frac{\left.\sigma\left(p_{\text {miss }}, E_{\text {miss }}\right)\right|_{\text {Raw }}}{C_{\text {Rad }}\left(p_{\text {miss }}, E_{\text {miss }}\right) \cdot C_{B M}\left(p_{\text {miss }}, E_{\text {miss }}\right)} \rightarrow \int_{E_{\text {miss }}} \rightarrow \times \frac{1}{C_{B C}\left(p_{\text {miss }}\right)} \rightarrow \sigma\left(p_{\text {miss }}\right) .
$$

The corrections applied to the absolute cross sections are shown in Fig. 3-35 and tabulated in Appendix C Tables C.6 and C.7.
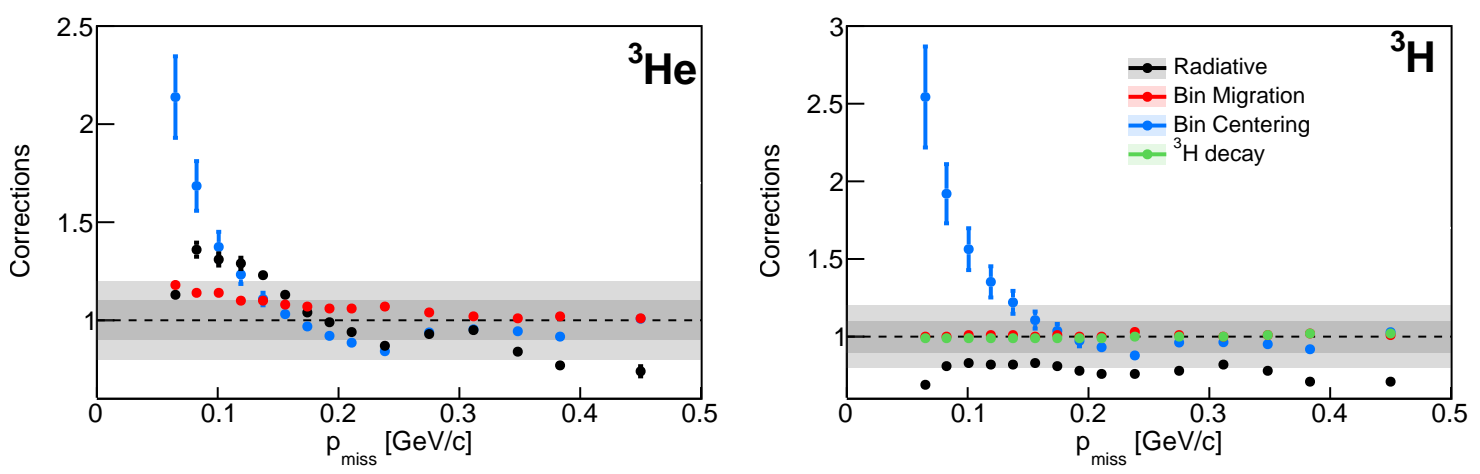

Figure 3-35: Size of corrections applied in the absolute analysis to obtain $\sigma_{3} \mathrm{He}$ (left) and $\sigma_{3} \mathrm{H}$ (right) respectively. The dark- and light-gray bands correspond to the 10 and $20 \%$ levels respectively.

\subsubsection{Systematic uncertainties}

The point-to-point systematic uncertainties due to the event-selection cuts were determined by repeating the analysis 100 times. As was done in the ${ }^{3} \mathrm{He} /{ }^{3} \mathrm{H}$ ratio analysis, for each iteration we chose each event-selection cut randomly (uniformly) within reasonable limits, see Table 3.7. We used the standard deviation of the resulting cross sections in each $p_{\text {miss }}$ bin as the systematic uncertainty from the event-selection cuts in that bin. These values range from $1 \%$ to $8 \%$ and are typically much smaller than the statistical uncertainties.

The uncertainty coming from the radiative correction was conservatively assigned to be $10 \%$ of the size of this correction for each $p_{\text {miss }}$ bin (after integrating over 
$\left.E_{m i s s}\right)$ and applied bin-by-bin. The uncertainty from the bin-migration correction was assigned exactly the same way. The bin-centering and decay correction uncertainties were described in sections 3.7 .4 and 3.7 .5 respectively. See Appendix C Tables C.6 and $\mathrm{C.7}$ for more details.

Additionally, there is an overall normalization uncertainty of $2.7 \%$ coming mainly from the target density uncertainty and HRS detection and trigger efficiencies. Other normalization uncertainties are $1 \%$ or less, as shown in Table 3.11 .

Table 3.11: Overall systematic uncertainties in the extraction of the ${ }^{3} \mathrm{He}\left(e, e^{\prime} p\right)$ and ${ }^{3} \mathrm{H}\left(e, e^{\prime} p\right)$ absolute cross sections. All uncertainties are summed in quadrature.

\begin{tabular}{c|c} 
& Overall Uncertainty \\
\hline \hline Target Walls & $\ll 1 \%$ \\
Target Density & $1.5 \%$ \\
Beam-Charge and Stability & $1 \%$ \\
Tritium Decay & $0.18 \%$ \\
HRS detection and trigger efficiencies & $2 \%$ \\
\hline Total & $\approx 2.7 \%$
\end{tabular}

\subsubsection{AV18 to CD-Bonn effective conversion}

Since the Cracow-group and CK+CC1 calculations used different $N N$-interaction models (see Table 3.12), we decided to study calculations carried out using a single $N N$ potential. We therefore rescaled the cross section determined from the factorized $\mathrm{CK}+C C 1$ model. In the absence of 2-dimensional $\left(p_{m i s s}, E_{m i s s}\right)$ spectral-function calculations with the CD-Bonn potential, we rescaled the $E_{\text {miss }}$-integrated cross section using the ratio of 1-dimensional momentum distributions calculated with the AV18+UIX and CD-Bonn+TM potentials from [126]:

The effective conversion factor $n^{\mathrm{CD}-\mathrm{Bonn}}\left(p_{\text {miss }}\right) / n^{\mathrm{AV} 18}\left(p_{\text {miss }}\right)$ is shown in Fig. 336. This correction effectively shifts the CK+CC1 $E_{\text {miss }}$-integrated cross-section from AV18 to the CD-Bonn potential. Consequently, the remaining differences can be attributed to intrinsic details of the cross-section model. 


$$
\sigma_{\mathrm{CK}+C C 1}^{\mathrm{CD}-\mathrm{Bonn}} \equiv \frac{n^{\mathrm{CD}-\mathrm{Bonn}}\left(p_{\text {miss }}\right)}{n^{\mathrm{AV} 18}\left(p_{\text {miss }}\right)} \times \sigma_{\mathrm{CK}+C C 1}^{\mathrm{AV} 18}
$$

Table 3.12: $N N$ potentials used in the different cross-section calculations.

\begin{tabular}{c|ccc} 
& Cracow & CK+CC1 & Sargsian \\
\hline \hline CD-Bonn & $\checkmark$ & & $\checkmark$ \\
AV18 & & $\checkmark$ & $\checkmark$
\end{tabular}

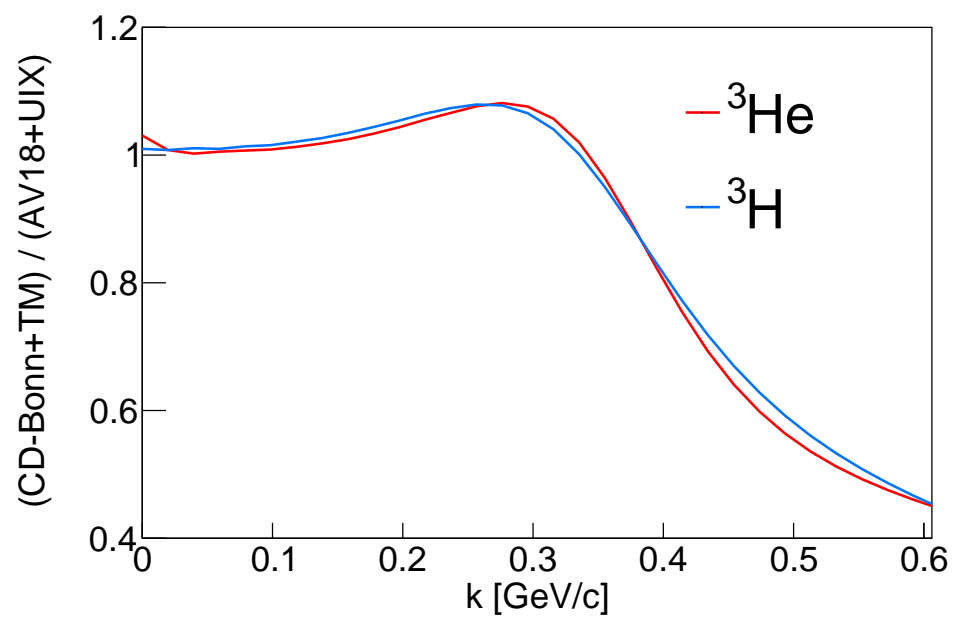

Figure 3-36: Ratio of the proton momentum distributions obtained using the CDBonn + TM and AV18+UIX potentials for ${ }^{3} \mathrm{He}$ and ${ }^{3} \mathrm{H}$ using the calculations from [126].

\subsubsection{Final results}

The resulting ${ }^{3} \mathrm{He}$ and ${ }^{3} \mathrm{H}$ absolute cross sections as a function of $p_{\text {miss }}$ (integrated over $\left.E_{\text {miss }}\right)$ extracted from the measured events and corrected for radiative, binmigration, bin-centering, and decay (in the case of ${ }^{3} \mathrm{H}$ ) effects following the procedure described in the previous section are summarized in Appendix C Tables C.6 and C.7 respectively, and shown in Fig. 3-37.

Fig. 3-37 also shows different cross-section calculations. These are obtained by calculating 70 differential cross-section values (38 and 32 in the low- and high- $p_{\text {miss }}$ kinematical settings respectively) in the kinematical points $\left(p_{m i s s}, E_{m i s s}, x_{B}, Q^{2}, \phi_{e^{\prime}}=\right.$ $0, \phi_{p}=180^{\circ}$ ) from Appendix C Tables C.3 and C.4 and subsequently summing over 
$E_{m i s s}$. The Sargsian-FSI cross section, which does not include $\mathrm{FSI}_{23}$, is only shown in the high- $p_{\text {miss }}$ kinematical setting where effects of $\mathrm{FSI}_{23}$ are smaller (see section 3.5.2).

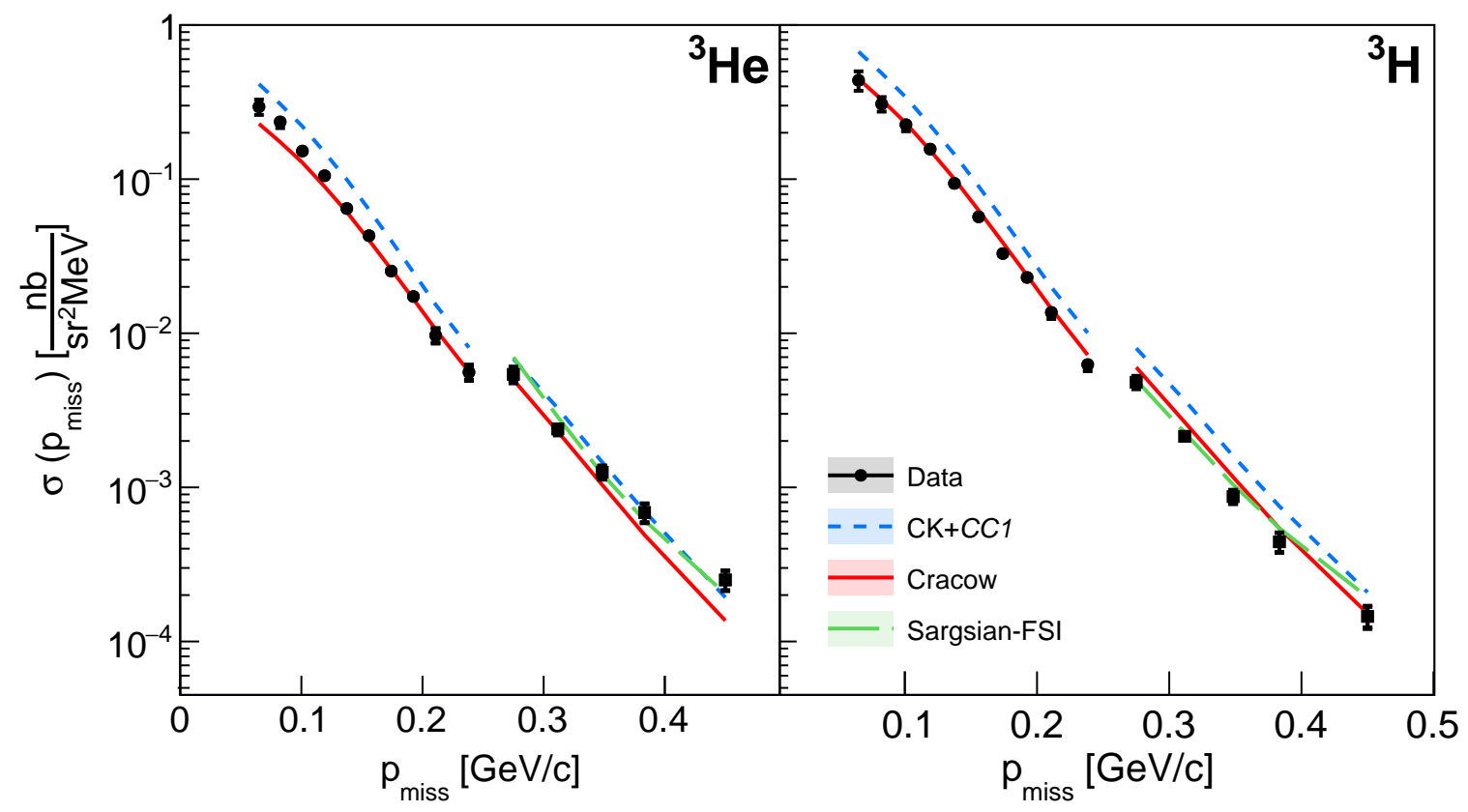

Figure 3-37: Absolute cross section as a function of $p_{\text {miss }}$ for ${ }^{3} \mathrm{He}$ (left) and ${ }^{3} \mathrm{H}$ (right). The markers correspond to the experimental cross sections for the low- $p_{m i s s}(\mathbf{\bullet})$ and high- $p_{\text {miss }}(\mathbf{\square})$ kinematical settings. The colored lines correspond to cross sections calculated using the theory models described in section 2.2.3 (all based on the CDBonn $N N$ potential).

Fig. 3-38 shows ratios of the experimental cross sections to the available PWIA cross sections for ${ }^{3} \mathrm{He}$ and ${ }^{3} \mathrm{H}$. In the case of ${ }^{3} \mathrm{H}$, the Cracow calculation agrees with the data within 10-20\% over the entire measured range, unlike for ${ }^{3} \mathrm{He}$, where they agree only in the range $150 \leq p_{\text {miss }} \leq 350 \mathrm{MeV} / c$. Outside this range, they disagree up to about a factor of $\sim 1.6$ in the highest $p_{\text {miss }}$ bin. The $\mathrm{CK}+C C 1$ calculation is on average $70 \%$ and $65 \%$ higher than the data for ${ }^{3} \mathrm{He}$ and ${ }^{3} \mathrm{H}$ respectively.

Fig. 3-38 (top) also shows the equivalent ratio from the most recent ${ }^{3} \mathrm{He}\left(e, e^{\prime} p\right)$ measurement, which was done at $Q^{2}=1.5\left(\mathrm{GeV} / c^{2}\right)^{2}$ and $x_{B}=1$ [42], to the PWIA calculation from [43]. As described in section 2.2.4, it was found that non-QE reaction mechanisms dominated the cross section and, consequently, the measured cross sections differed from PWIA calculations by up to factors of $\sim 2$ and $\sim 3$ for $p_{\text {miss }}<250$ 

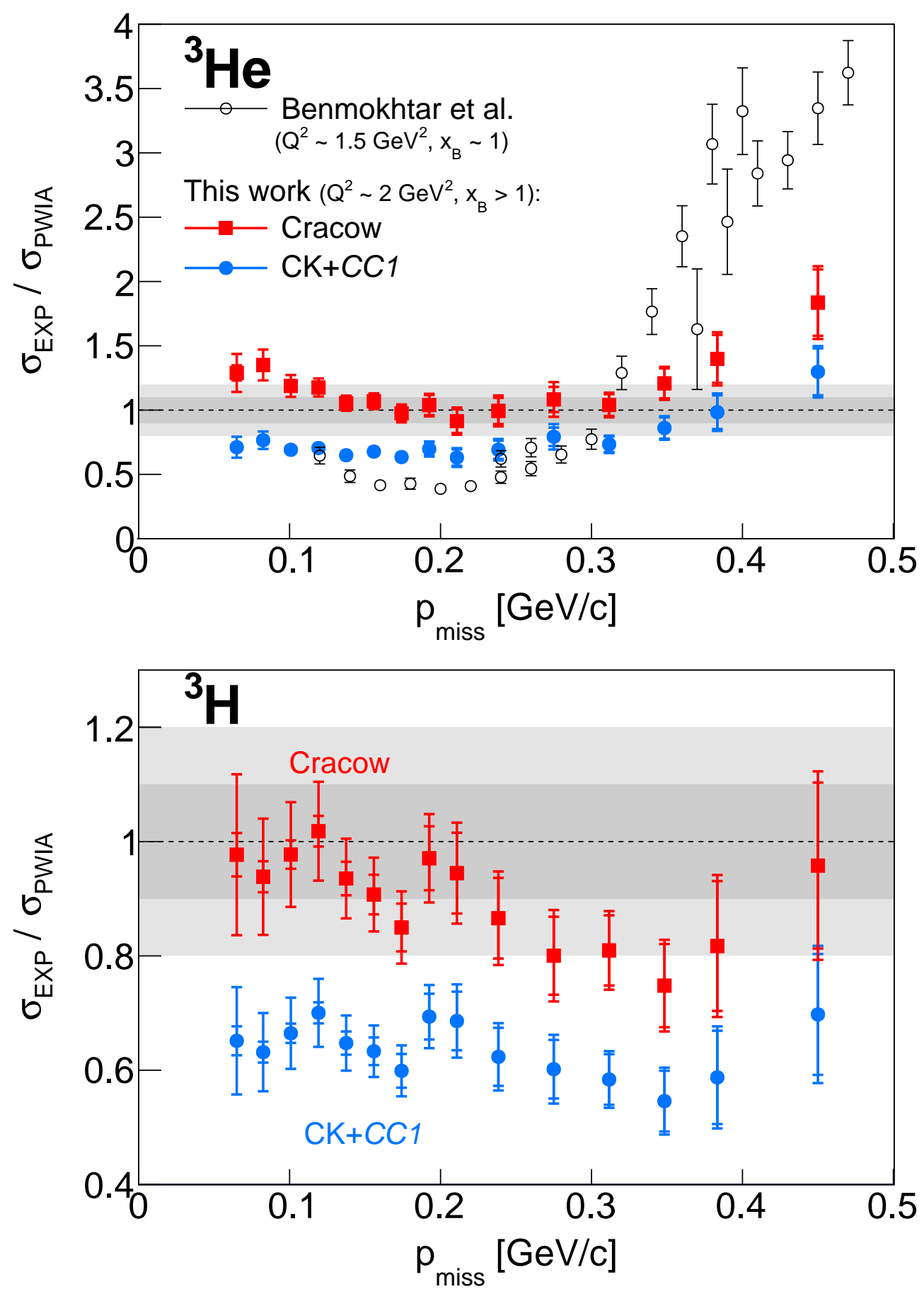

Figure 3-38: Ratio of the experimental cross section to different PWIA calculations for ${ }^{3} \mathrm{He}\left(\right.$ top) and ${ }^{3} \mathrm{H}$ (bottom). Ratios to the Cracow (-) and $\mathrm{CK}+C C 1(\bullet)$ models are shown in red and blue respectively. Also shows as open markers $(\bigcirc)$ on the top plot are the corresponding ratios from [42], measured at lower $Q^{2}$ and $x_{B}=1$ kinematics, to the PWIA calculation from [43. The dark- and light-gray bands correspond to the 10 and $20 \%$ agreement intervals respectively. 
$\mathrm{MeV} / c$ and $400<p_{\text {miss }}<500 \mathrm{MeV} / c$ respectively. The large contribution of non-QE reaction mechanisms limited their ability to constrain the nucleon momentum distribution. These effects are much smaller in our measurement, due to our kinematics selection.

In order to estimate the effect of the struck-nucleon rescattering (FSI), we also compared the measurement to the Sargsian-FSI calculation in the high- $p_{\text {miss }}$ kinematical setting, see Fig. 3-39.

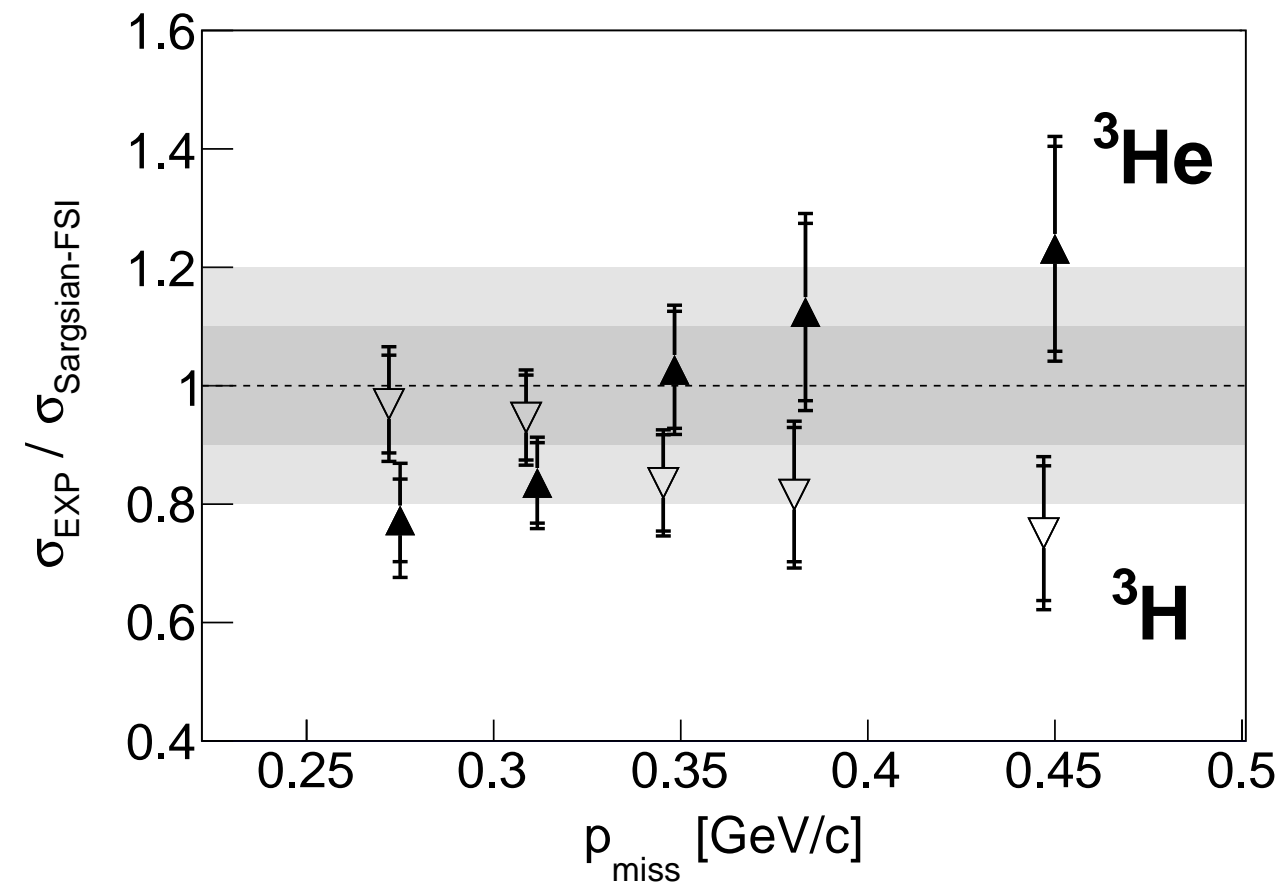

Figure 3-39: Ratio of the experimental cross section to the Sargsian-FSI calculations, which include rescattering of the struck nucleon but do not include $\mathrm{FSI}_{23}$, for ${ }^{3} \mathrm{He}$ $(\boldsymbol{\Delta})$ and ${ }^{3} \mathrm{H}(\nabla)$ in the high- $p_{m i s s}$ kinematical setting. The dark- and light-gray bands correspond to the 10 and $20 \%$ agreement intervals respectively.

The inclusion of FSI enhances the agreement with respect to the PWIA calculations. The ratio of data to FSI calculation increases with $p_{\text {miss }}$ for ${ }^{3} \mathrm{He}$ and decreases for ${ }^{3} \mathrm{H}$. This result can be qualitatively explained in terms of SCX following the reasoning given at the end of section 3.6.8. This hypothesis is further supported by the observation that the isoscalar $A=3$ cross section (i.e. $\sigma_{3} \mathrm{He}+\sigma_{3 \mathrm{H}}$ ) is well described by the calculations to within the accuracy of our data over the entire $p_{\text {miss }}$ range, since SCX effects are expected to be suppressed in isoscalar systems where $p n$-SCX and 
np-SCX largely cancel (See Fig. 3-40). Thus, these results suggest that the most plausible explanation for the high- $p_{\text {miss }}$ discrepancy found in the ${ }^{3} \mathrm{He} /{ }^{3} \mathrm{H}$-ratio analysis is SCX. Nevertheless, additional calculations that quantify the effect of SCX are necessary to confirm this hypothesis. The isoscalar agreement between the calculations and the data validates current models of the $A=3$ system up to initial momenta of $500 \mathrm{MeV} / c$.

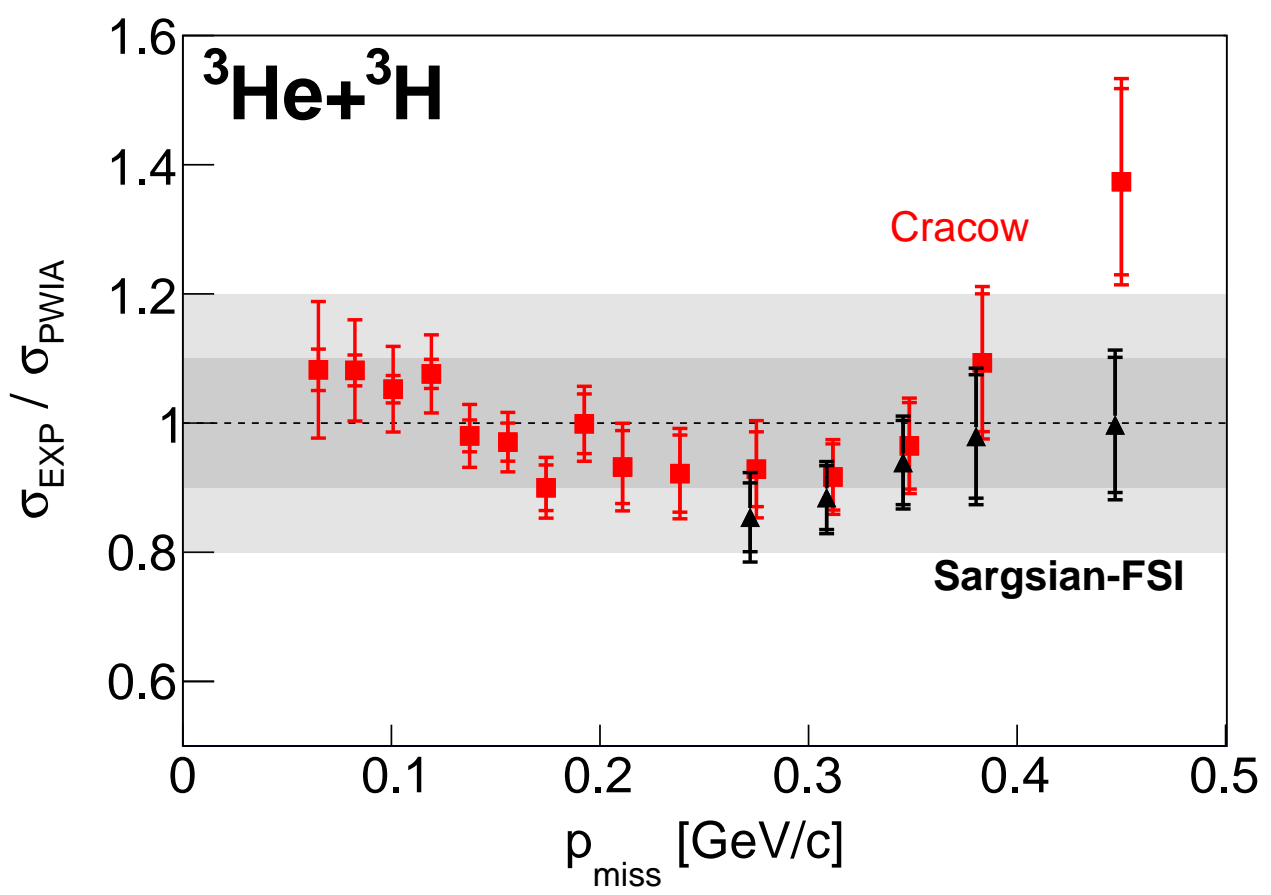

Figure 3-40: Ratio of the total $A=3$ experimental cross section $\left({ }^{3} \mathrm{He}+{ }^{3} \mathrm{H}\right)$ to different calculations as a function of missing momentum. The dark- and light-gray bands correspond to the 10 and $20 \%$ agreement intervals respectively. 


\section{Chapter 4}

\section{Generalized Contact Formalism}

[Phys. Lett. B 780, 211 (2018), arXiv:1907.03658 (2019), Phys. Lett. B 785, 304 (2018)]

\subsection{Introduction}

As explained in chapter 1, the traditional nuclear-structure effective models (e.g. the Independent-Particle Shell-Model) fail to describe the dynamic effects of ShortRange Correlations in nuclei. Developing a complementary model to describe SRCs is fundamental to obtain a full description of the nucleus. In this chapter, I will overview an effective model that describes the high-momentum and short-distance components of the nuclear wave function. This model is based on the (atomic) Contact Formalism, which describes a system of two-component fermions interacting via a short-range interaction and has been validated in the case of ultra-cold atomic gases (see, e.g., [133, 134, 135, 136]). The original GCF derivation comes from [137]. My work focused on testing the GCF against QMC distributions, extracting contacts with proper systematic uncertainties [138, studying their scale and scheme dependence and short-distance/high-momentum equivalence [139], and extending the GCF to calculations of correlation functions [140]. The QMC distributions against which we test the GCF were calculated by [11, 16, 66, 73, 141, 142]. 


\subsection{Contact Formalism in atomic systems}

In the (atomic) Contact Formalism, the many-body wave function for two-component Fermi gases with short-range interactions (i.e. range of the interaction much smaller than the relevant scales of the system such as its scattering length and typical interparticle distances) is expressed as a factorized product of an asymptotic pair wave function $(\varphi)$, and a function $(A)$ which describes the interaction of the pair as a whole with the residual system [143].

$$
\Psi \underset{r_{i j} \rightarrow 0}{\longrightarrow} \varphi\left(\vec{r}_{i j}\right) A\left(\vec{R}_{i j},\left\{\vec{r}_{k}\right\}_{k \neq i, j}\right) .
$$

Here, $\vec{r}_{i j}=\left(\vec{r}_{i}-\vec{r}_{j}\right) / 2$, and $\vec{R}_{i j}=\vec{r}_{i}+\vec{r}_{j}$ are the relative and center-of-mass coordinates of the pair, and $\left\{\vec{r}_{k}\right\}_{k \neq i, j}$ refers to the coordinates of all the particles from the residual system. The pairing at short distance will happen predominantly between $l=0$ (swave) pairs, since the centrifugal forces make the wave function very small for higher partial waves. In the zero-range model [144], the asymptotic pair wave function becomes:

$$
\varphi\left(\vec{r}_{i j}\right)=1 / r_{i j}-1 / a
$$

where $a$ is the scattering length. This can be used to determine the 1-body momentum distribution of the system $n(\vec{k})$. Given that $n(\vec{k}) \sim|\tilde{\Psi}|^{2}$ (where $\tilde{\Psi}$ is the Fourier transform of $\Psi)$ and $\int d^{3} r e^{-i \vec{k} \cdot \vec{r}} \frac{1}{r}=\frac{4 \pi}{k^{2}}$, we get:

$$
n(\vec{k})=\frac{C}{k^{4}}
$$

where $C \sim\langle A \mid A\rangle$ is a constant called the "contact" and defines the thermodynamical properties of these gases [145]. 


\subsection{Generalization to nuclear systems}

Nuclear systems do not fulfill the conditions of the Contact Formalism. The range of the short-distance interaction $\left(r_{\text {eff }} \sim \hbar / 2 m_{\pi} c \approx 0.7 \mathrm{fm}\right.$, where $m_{\pi}$ is the pion mass) is less than, but not much less than the scattering lengths $\left(a\left({ }^{3} S_{1}\right)=5.42 \mathrm{fm}\right)$ and average nucleon-nucleon distances $\left(d=(\rho / 2)^{-1 / 3} \approx 2.3 \mathrm{fm}\right)$. Additionally, unlike the two-component atomic gases, nuclei are not precisely two-component systems, since different types of pairs can be formed with different combinations of protons and neutrons with different spin states.

However, nuclear systems do exhibit several similarities to two-component ultracold atomic gases [146]. For example, nuclei and atomic gases can have the same dimensionless interaction strength $\left(k_{F} \cdot a\right)^{-1}$. Even if the similarities between the two systems are accidental, these facts make the Contact Formalism a worthwhile candidate theory to model and understand Short-Range Correlations in nuclei.

To take into account the fact that nuclei are not two-component Fermi systems, the expression from equation 4.1 can be written more generally as:

$$
\Psi \underset{r_{i j} \rightarrow 0}{\longrightarrow} \sum_{\alpha} \varphi_{N N}^{\alpha}\left(\vec{r}_{i j}\right) A_{N N}^{\alpha}\left(\vec{R}_{i j},\left\{\vec{r}_{k}\right\}_{k \neq i, j}\right)
$$

where $N N$ denotes the type of pair ( $p p, p n$, or $n n)$, and $\alpha$ refers to the spin of the pair. This way, we take into account the contribution from different partial waves. Previous applications of the Contact Formalism to nuclear systems have focused on the dominant channel: the deuteron-like pairs $(p n, l=0,2$ and $s=1$ coupled to $j=1)$ [52, 146, 147]. The Contact Formalism was extended to also include the singlet $p p$, $p n$, and $n n$ s-wave channel $(l=s=j=0)$. Pairs such as $p p$ or $n n$ with $s=1$ should have a negligible contribution, since these configurations of short-distance pairs are blocked by the Pauli principle.

The pair asymptotic wave function $\varphi_{N N}^{\alpha}\left(\vec{r}_{i j}\right)$ is taken as the zero-energy numerical solution of the Schrödinger equation for a pair of nucleons $N N$ with spin $\alpha$ in three dimensions. The obtained wave functions are insensitive to the exact value of the energy at small distances and high momenta. Since the Hamiltonian in the Schrödinger 
equation depends on the potential, different models for the $N N$ potential yield different functional forms for the $N N$ asymptotic wave function. Fig. 4-1 shows the resulting $\left|\varphi_{N N}^{\alpha}\right|^{2}$ calculated using the AV18 potential. Fig. 4-10 shows the resulting $\left|\varphi_{N N}^{\alpha}\right|^{2}$ obtained from different models of the $N N$ potential. For a given $N N$ potential, these asymptotic wave functions should be universal (meaning nucleus-independent), because when $N N$ pairs interact at very short distances, their interaction is very localized, and therefore independent of the nucleus in which it occurs.
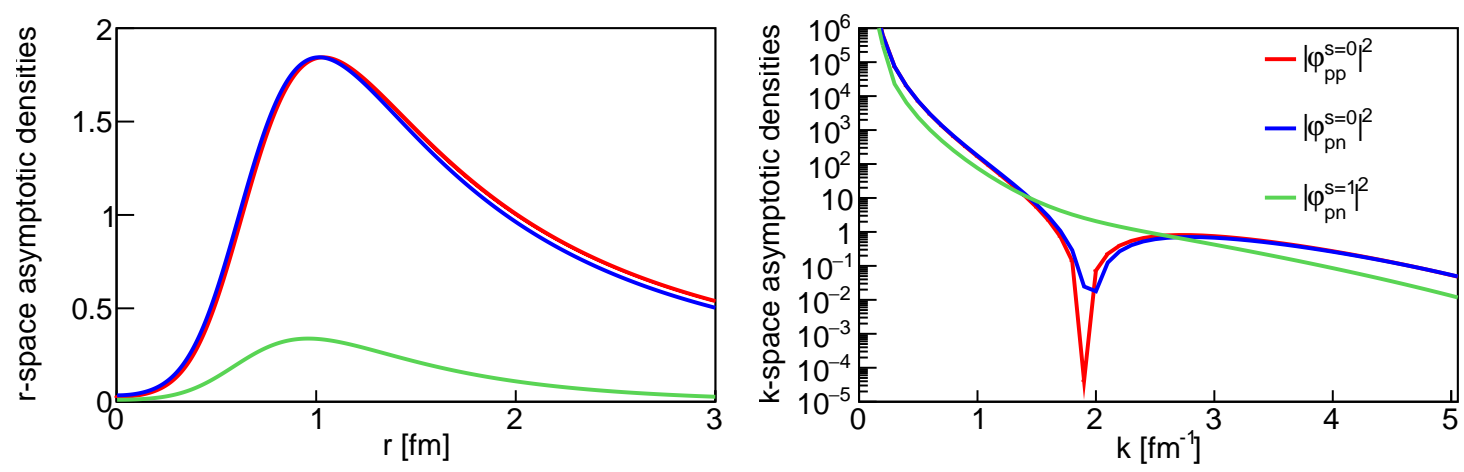

Figure 4-1: $N N$ asymptotic densities calculated using the AV18 $N N$ potential. The left and right panels show these distributions calculated in coordinate and momentum space respectively. Note that the spin-0 functions are both qualitatively and quantitatively similar.

\subsubsection{Coordinate densities and momentum distributions}

Just like in the atomic case, the wave function from the GCF can be used to write down 1- and 2-body coordinate densities and momentum distributions [137. For example, the 2-body coordinate density at short distances can be written as:

$$
\rho_{N N}^{\alpha}(\vec{R}, \vec{r}) \underset{r \rightarrow 0}{\longrightarrow} C_{N N}^{\alpha}(\vec{R})\left|\varphi_{N N}^{\alpha}(\vec{r})\right|^{2},
$$

where $\vec{R}=\vec{R}_{i j}, \vec{r}=\vec{r}_{i j}$, and the $i j$ indices have been dropped. $N N$ denotes the nucleon pair ( $p p, p n$, or $n n)$. Here, the contacts are diagonal matrices that correspond to $C_{N N}^{\alpha}=N_{N N}\left\langle A_{N N}^{\alpha} \mid A_{N N}^{\alpha}\right\rangle$, where $N_{N N}$ is the number of $N N$ pairs. We have assumed that the contacts are averaged over the nuclear magnetic projection. The deuteron-channel contact $C_{p n}^{s=1}$ is the sum over the three diagonal deuteron contacts 
$(m= \pm 1,0)$. Similarly, one can write the 2-body momentum distribution as:

$$
F_{N N}^{\alpha}(\vec{Q}, \vec{q}) \underset{q \rightarrow \infty}{\longrightarrow} C_{N N}^{\alpha}(\vec{Q})\left|\varphi_{N N}^{\alpha}(\vec{q})\right|^{2}
$$

where $\vec{Q}=\vec{k}_{i}+\vec{k}_{j}$ and $\vec{q}=\left(\vec{k}_{i}-\vec{k}_{j}\right) / 2$ are respectively the center-of-mass and relative momentum of the pair, and $\varphi_{N N}^{\alpha}(\vec{q})$ is the Fourier transform of $\varphi_{N N}^{\alpha}(\vec{r})$.

Fig. 4-2 shows the Oxygen-16 QMC 2-body coordinate densities $\rho_{N N}^{16} \mathrm{O}(R, r)$ for $p n$ and $p p$ pairs calculated with the $\mathrm{N}^{2} \mathrm{LO}(1.0 \mathrm{fm})$ and $\mathrm{AV}^{\prime}+\mathrm{UIX}_{\mathrm{c}}$ potentials. These distributions have been scaled to have the same value at $r \sim 1 \mathrm{fm}$ to emphasize the short-distance factorization expected from the GCF. By integrating equation 4.5 over $r$ up to $1 \mathrm{fm}$, the ${ }^{16} \mathrm{O}$ contacts $C_{N N}^{\alpha}(R)$ can be determined. The result is shown in Fig. 4-3. The resulting contacts are compared to contacts obtained from uncorrelated 2-body coordinate densities determined by doing a convolution of the single-nucleon density distribution with itself:

$$
\rho_{N N, \text { un-corr }}^{16}(R) \equiv \int_{0}^{1 \mathrm{fm}} d \Omega_{R} d \vec{r} \rho_{N}^{16} \mathrm{O}(\vec{R}+\vec{r} / 2) \rho_{N}^{16} \mathrm{O}(\vec{R}-\vec{r} / 2),
$$

and adding the effects of the Pauli principle in the case of $p p$ pairs (see section 4.7.3). That the contacts from QMC calculations agree very well with the contacts from uncorrelated 2-body densities implies that these quantities are long-range Mean-Field properties of nuclei. The observed agreement is insensitive to the integration limit for $r$ from zero to $1 \mathrm{fm}$.

Since $\rho_{N N}(R, r)$ and $F_{N N}(Q, q)$ QMC calculations are computationally demanding and thus not available for many nuclei and $N N$ potentials, we further study the GCF analyzing QMC coordinate densities and momentum distributions integrated over $R$ and $Q$ respectively. In the $\mathrm{GCF}$, only the contacts depend on these variables and, consequently, the nuclear contact coefficients correspond to:

$$
\begin{aligned}
C_{N N}^{\alpha} & =\int d \vec{R} C_{N N}^{\alpha}(R), \\
C_{N N}^{\alpha} & =\int d \vec{Q} C_{N N}^{\alpha}(Q),
\end{aligned}
$$




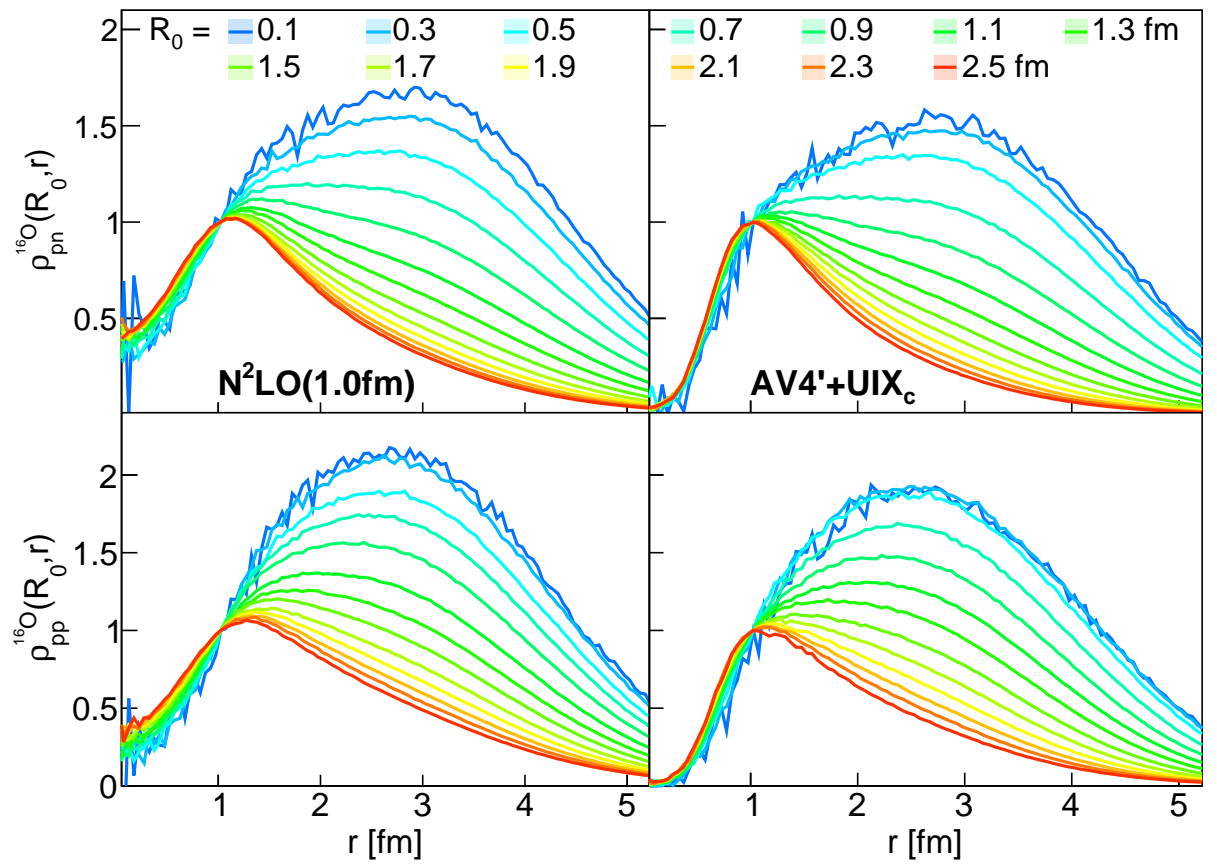

Figure 4-2: QMC Oxygen-16 2-body coordinate densities $\rho_{N N}^{16} \mathrm{O}\left(R_{0}, r\right)$ for $p n$ (top) and $p p$ (bottom) pairs calculated with the $\mathrm{N}^{2} \mathrm{LO}(1.0 \mathrm{fm})$ (left) and $\mathrm{AV} 4^{\prime}+\mathrm{UIX}_{\mathrm{c}}$ (right) potentials. The different colors correspond to different values of $R_{0}$. All calculations are scaled to have the same value at $r \sim 1 \mathrm{fm}$ to emphasize the short-distance factorization.

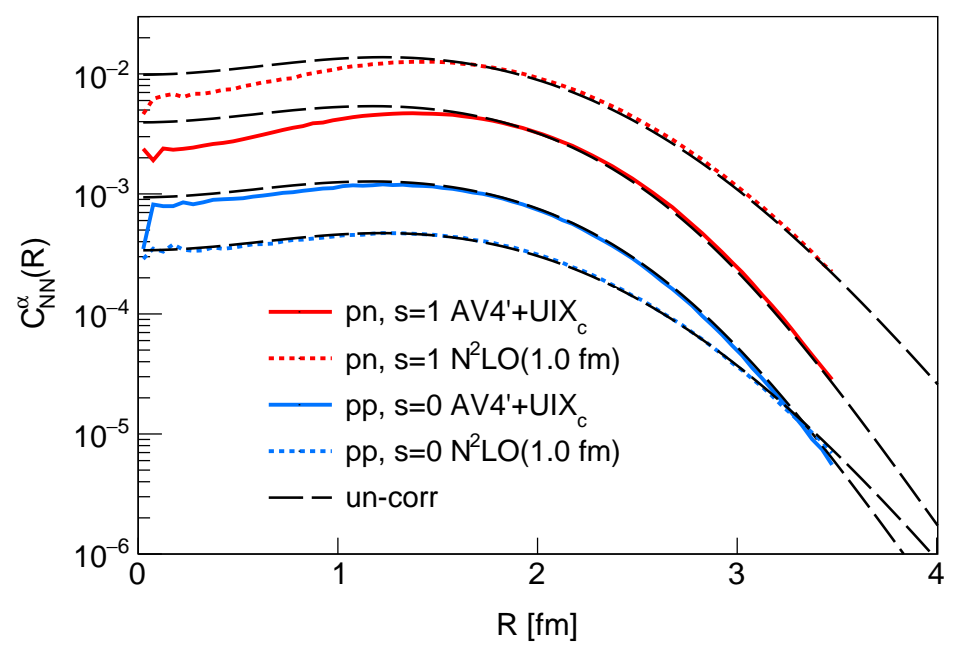

Figure 4-3: Oxygen-16 contacts $C_{N N}^{\alpha}(R)$. The colored lines are obtained by integrating QMC 2-body coordinate densities $\rho_{N N}^{\alpha}(R, r)$ over $r$ from 0 to $1 \mathrm{fm}$. The solid and dotted (-.-.-) lines correspond to contacts determined from distributions calculated with the AV4' $+\mathrm{UIX}_{\mathrm{c}}$ and $\mathrm{N}^{2} \mathrm{LO}(1.0 \mathrm{fm}) N N$ potentials respectively. The red and blue lines correspond to the $p n, s=1$ and $p p, s=0$ contacts respectively. The black dashed (- - ) lines correspond to the equivalent quantity determined from uncorrelated 2-body coordinate densities. See text for details. 
in coordinate and momentum space respectively. Accordingly, the integrated 2-body coordinate density corresponds to:

$$
\rho_{N N}^{\alpha}(\vec{r}) \underset{r \rightarrow 0}{\longrightarrow} C_{N N}^{\alpha}\left|\varphi_{N N}^{\alpha}(\vec{r})\right|^{2},
$$

and the 2-body momentum distribution corresponds to:

$$
F_{N N}^{\alpha}(\vec{q}) \underset{q \rightarrow \infty}{\longrightarrow} C_{N N}^{\alpha}\left|\varphi_{N N}^{\alpha}(\vec{q})\right|^{2},
$$

For example, in the GCF the proton-proton 2-body momentum distribution is:

$$
F_{p p}(\vec{q})=C_{p p}^{s=0}\left|\varphi_{p p}^{s=0}(\vec{q})\right|^{2},
$$

since the only significant contribution at high-momentum is expected to be $s=0$. The neutron-neutron 2-body momentum distribution has the same expression after replacing $p p$ with $n n$. The total $p n$ 2-body momentum distribution is:

$$
F_{p n}(\vec{q})=C_{p n}^{s=0}\left|\varphi_{p n}^{s=0}(\vec{q})\right|^{2}+C_{p n}^{s=1}\left|\varphi_{p n}^{s=1}(\vec{q})\right|^{2}
$$

since it also has a spin-1 contribution. Since the 1-body momentum distribution should be dominated by SRCs at high-momentum, it can be written as the sum of all the relevant 2-body contributions. For example, the proton 1-body momentum distribution is:

$$
\begin{aligned}
n_{p}(\vec{k}) & =2 F_{p p}(\vec{k})+F_{p n}(\vec{k}) \\
& =2 C_{p p}^{s=0}\left|\varphi_{p p}^{s=0}(\vec{k})\right|^{2}+C_{p n}^{s=0}\left|\varphi_{p n}^{s=0}(\vec{k})\right|^{2}+C_{p n}^{s=1}\left|\varphi_{p n}^{s=1}(\vec{k})\right|^{2},
\end{aligned}
$$

where $\vec{k}$ is the single-nucleon momentum. The neutron 1-body momentum distribution is obtained by replacing all the $p$ indices by $n$ and vice versa. Thus, according to the GCF, in the regions where SRCs dominate (namely short-distances and highmomenta), the full nuclear densities can be described by a series of universal functions 
that carry the coordinate or momentum dependence, times a series of constants (i.e. the contacts) which carry the nucleus dependence.

\subsubsection{Normalization conventions}

The relative normalization between the $\varphi_{N N}^{\alpha}$ 's and the contacts can be shifted from one another to give the same overall normalization in equations 4.10 and 4.11 . In this work, we chose to normalize the asymptotic $N N$ wave functions such that:

$$
\int_{k_{F}}^{\infty}\left|\varphi_{N N}^{\alpha}(\vec{k})\right| d \vec{k}=1
$$

Therefore, integrating the total 1-body momentum distribution $n(\vec{k})=n_{p}(\vec{k})+n_{n}(\vec{k})$ from $k_{F}$ to $\infty$ we get:

$$
\int_{k_{F}}^{\infty} n(\vec{k}) d \vec{k}=2\left(C_{p p}^{s=0}+C_{n n}^{s=0}+C_{p n}^{s=0}+C_{p n}^{s=1}\right) .
$$

Since: $\int_{0}^{\infty} n(\vec{k}) d \vec{k}=A$, where $A$ is the number of nucleons, the contacts (divided by $A / 2$ ) represent the fraction of high-momentum nucleons in a given nucleus:

$$
\frac{\int_{k_{F}}^{\infty} n(\vec{k}) d \vec{k}}{\int_{0}^{\infty} n(\vec{k}) d \vec{k}}=\frac{C_{p p}^{s=0}+C_{n n}^{s=0}+C_{p n}^{s=0}+C_{p n}^{s=1}}{A / 2}
$$

Thus, $C_{N N}^{\alpha} /(A / 2)$ gives the fraction of the momentum distribution corresponding to high-momentum $N N, \alpha$ pairs. The normalization of $\varphi_{N N}^{\alpha}(\vec{r})$ in coordinate space comes naturally from taking the Fourier transforms of $\varphi_{N N}^{\alpha}(\vec{k})$ normalized according to equation 4.15

\subsection{Benchmarking the GCF}

As expressed in equations 4.10 and 4.11 , once the asymptotic (nucleus-independent) $N N$ densities are calculated, only the contacts are needed to describe the many-body nuclear densities for a given nucleus at short-distances or high-momenta. In this 
section, I will demonstrate the validity of this prediction, and then describe different ways of extracting these contacts. We will use only the AV18 $N N$ potential in this section.

\subsubsection{Contacts from 2-body coordinate densities}

I extracted the nuclear contacts by fitting the factorized 2-body coordinate density from equation 4.10 to the corresponding VMC 2-body coordinate density from [16, 66, [148] at short distances. Fig. 4-4 shows the normalized VMC pn, pp and $n n$ 2-body coordinate densities for $3 \leq A \leq 40$. There are two regimes: 1) the nucleus-dependent long-distance $(r>1 \mathrm{fm})$ region which is governed by single-particle behavior, and 2) the nucleus-independent short-distance $(r<1 \mathrm{fm})$ region, which is dominated by SRCs.
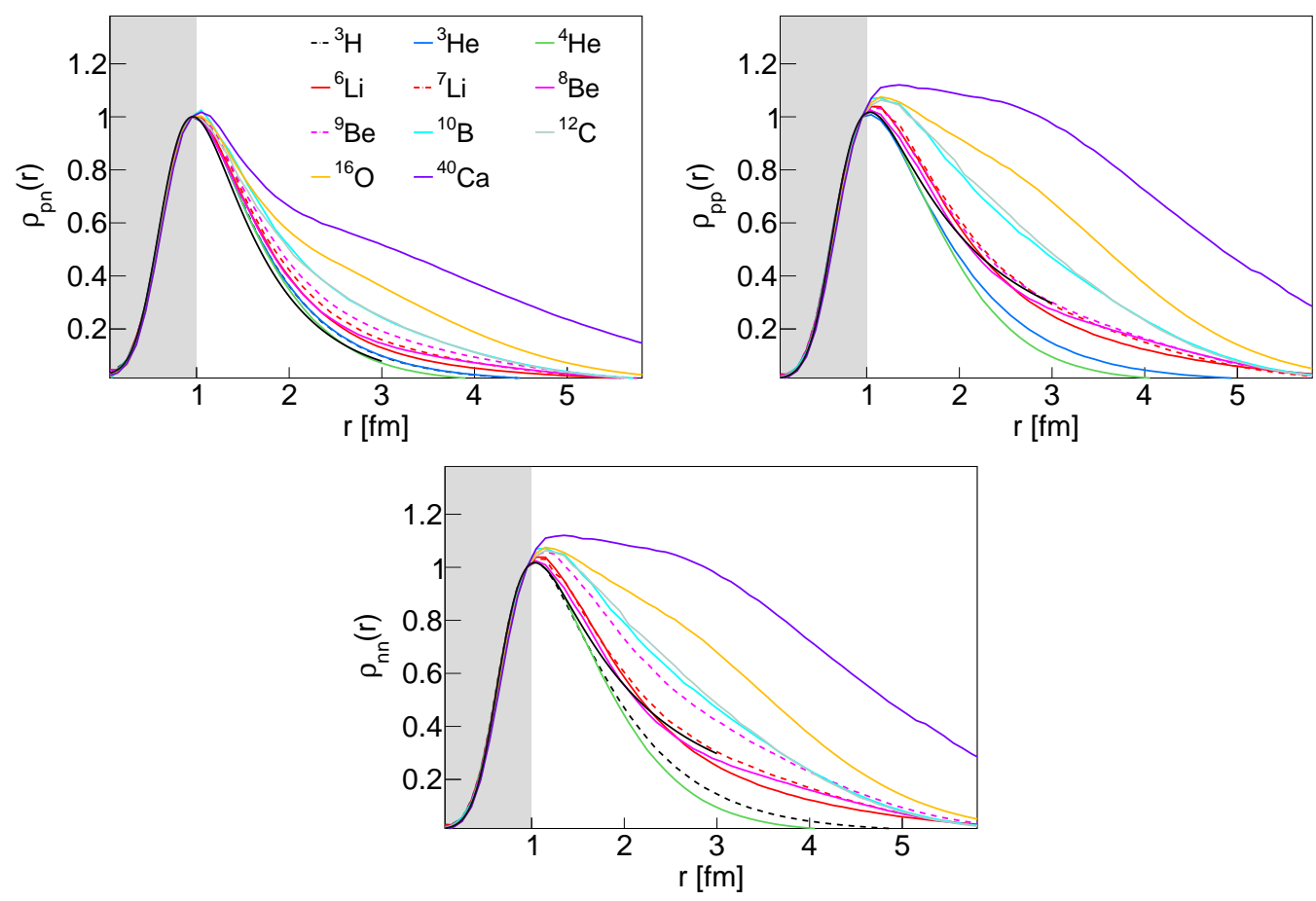

Figure 4-4: Short-distance universality of AV18 2-body coordinate densities. The different colored lines correspond to VMC calculations for different nuclei carried out using the AV18+UX potential. All these distributions are normalized to have the same value at $r \approx 1.0 \mathrm{fm}$. Also shown as black-solid lines are the $N N$ asymptotic densities $\left|\varphi_{N N}^{\alpha}\right|^{2}$ for the corresponding channel. The gray area shows the range in which SRCs dominate the 2-body coordinate densities. 
Fig. 4-4 also shows the $N N$ asymptotic densities $\left|\varphi_{N N}^{\alpha}\right|^{2}$ for the corresponding channel. The short-distance behavior agrees with all nuclei, and the long-distance behavior does not. This validates the GCF prescription for 2-body densities at short distances (equation 4.10) in the case of the AV18 $N N$ potential. The scale factors used to normalize the $\mathrm{VMC}$ densities (to have the same value at $1 \mathrm{fm}$ ) are the contacts.

We extracted the coordinate-space contacts $\left(C_{p p}^{s=0}, C_{n n}^{s=0}, C_{p n}^{s=0}\right.$, and $\left.C_{p n}^{s=1}\right)$ by doing a simultaneous fit to $\rho_{p p}(r), \rho_{n n}(r)$ (in the case of symmetric nuclei, $\rho_{n n}(r) \equiv \rho_{p p}(r)$ ), and $\rho_{p n}(r)$. Since $\rho_{p n}(r)$ has contributions from both $C_{p n}^{s=0}$ and $C_{p n}^{s=1}$, and spin-isospin projections have not been calculated, we assumed isospin symmetry $\left(C_{p p}^{s=0}=C_{n n}^{s=0}\right.$ $=C_{p n}^{s=0}$ for the symmetric nuclei). This assumption is evaluated in the next section. The fits to extract the contacts are carried out in the range $0.25 \leq r \leq 1.0 \mathrm{fm}$. The contact uncertainties are extracted by varying the fit limits by $\pm 0.25 \mathrm{fm}$. The extracted AV18 contacts are shown in Table 4.1 under "r-space".

\subsubsection{Contacts from 2-body momentum distributions}

We follow an almost identical procedure to extract nuclear contacts in momentum space. We extracted the nuclear contacts by fitting the factorized 2-body momentum distributions from equation 4.11 to the corresponding VMC 2-body momentum distributions from [16, 66, 148] at high momenta. Fig. 4-5 shows the ratios of $p n$, $p p$, and $n n$ 2-body momentum distributions for $3 \leq A \leq 40$ to the $N N$ asymptotic densities $\left|\varphi_{N N}^{\alpha}\right|^{2}$ for the corresponding channel normalized so that they all have the same value at $q \approx 4.5 \mathrm{fm}^{-1}$.

The high-momentum behavior agrees for all nuclei, and the low-momentum behavior does not. This validates the GCF prescription for 2-body distributions at high momenta (equation 4.11). The scale factors used to normalize the VMC densities to all agree at $q \approx 4.5 \mathrm{fm}^{-1}$ are the contacts.

We extracted the momentum-space contacts $\left(C_{p p}^{s=0}, C_{n n}^{s=0}, C_{p n}^{s=0}\right.$, and $\left.C_{p n}^{s=1}\right)$ by simultaneously fitting $F_{p p}(q), F_{n n}(q)$ (in the case of symmetric nuclei, $F_{n n}(q) \equiv F_{p p}(q)$ ), $F_{p n}(q)$, and $F_{S T=10}(q)$. In the case of the $F_{S T=10}(q)$ distribution, we assume that, at high-momentum, this function is only due to $p n, s=1$ pairs. In this case, we do not 


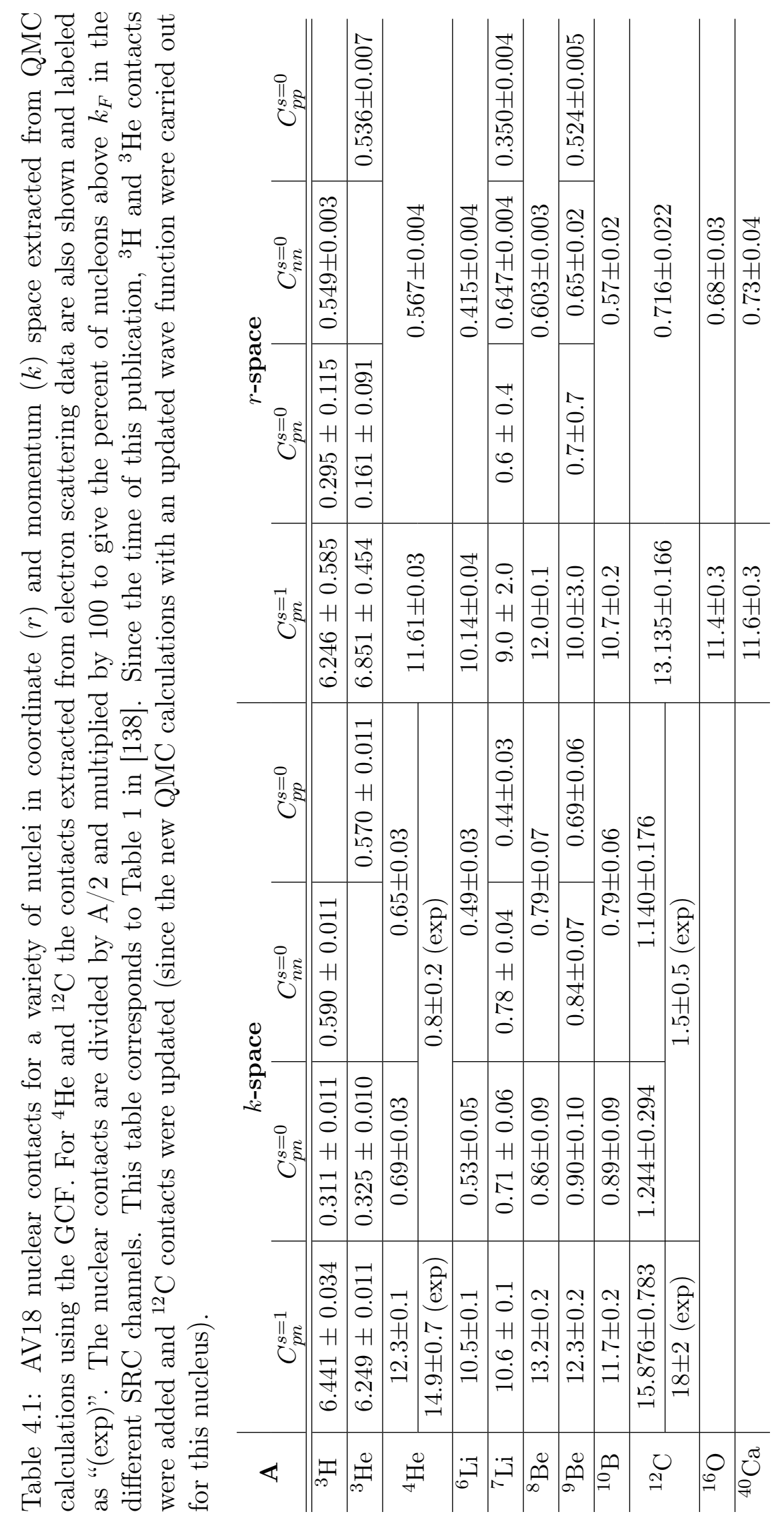



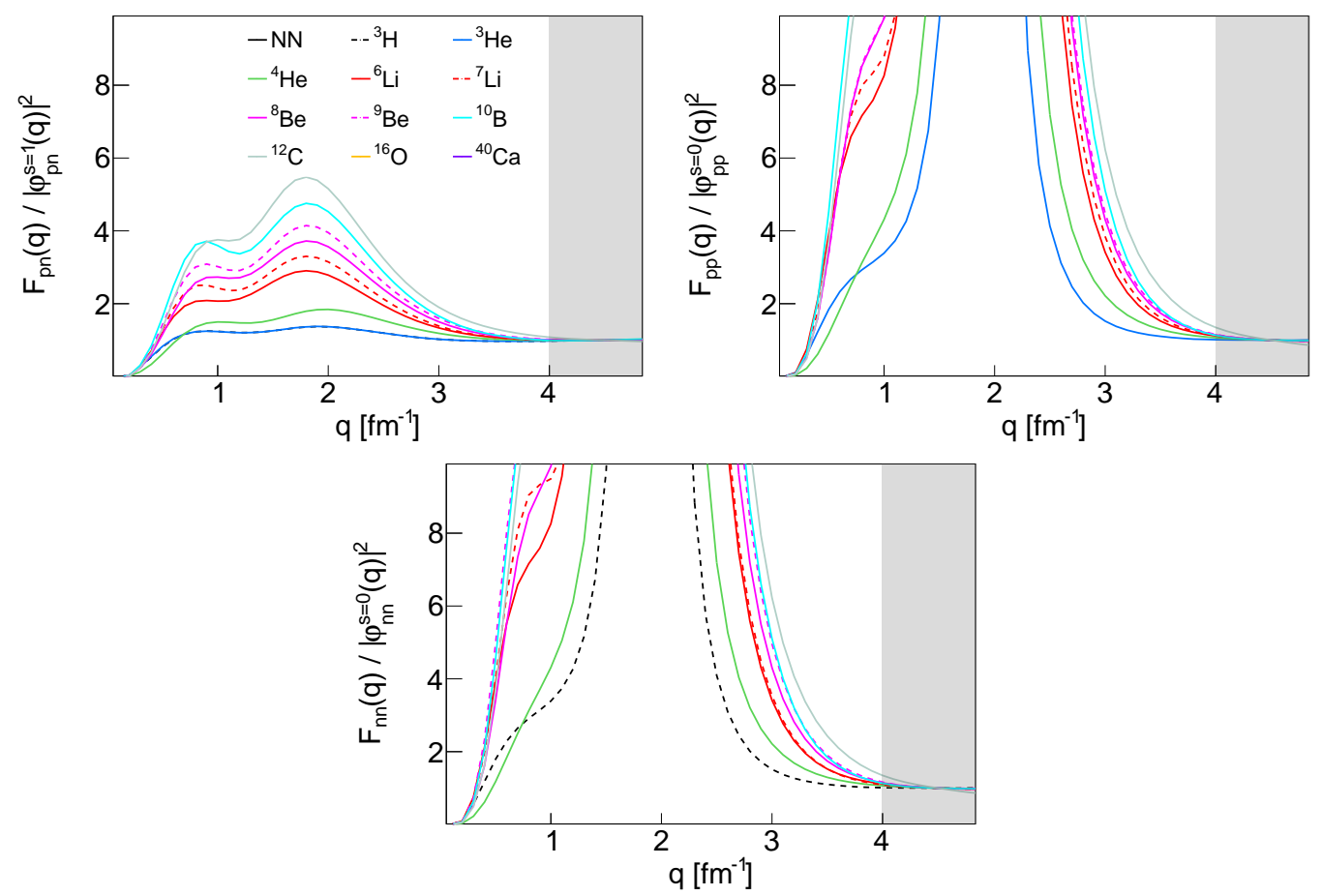

Figure 4-5: High-momentum universality of AV18 2-body momentum distributions. The different colored lines correspond to VMC calculations for different nuclei carried out using the AV18+UX potential, divided by the $N N$ asymptotic densities $\left|\varphi_{N N}^{\alpha}\right|^{2}$ for the corresponding channel. All these distributions are normalized to have the same value at $q \approx 4.5 \mathrm{fm}^{-1}$ in order to visualize the high-momentum universality. The gray area shows the range in which SRCs dominate the 2-body momentum distributions. 
assume isospin symmetry $\left(C_{p p}^{s=0}=C_{n n}^{s=0}=C_{p n}^{s=0}\right.$ for the symmetric nuclei), and we use the results to test it. The fits to extract the contacts are carried out in the range $4.0 \leq q \leq 4.8 \mathrm{fm}^{-1}$. The contact uncertainties are extracted by varying the fit limits by $\pm 0.2 \mathrm{fm}^{-1}$. The extracted AV18 contacts are shown in Table 4.1 under " $k$-space".

\section{Delayed scaling in 2-body momentum distributions}

The scales for SRCs in coordinate and momentum spaces are the nucleon radius $\left(r_{N}\right)$ and the Fermi momentum $\left(k_{F}\right)$ respectively. That is, in coordinate space, SRCs are predicted to dominate for $r \lesssim r_{N}$. Similarly, in momentum space, SRCs are predicted to dominate for $q \gtrsim k_{F}$. The first statement was verified in section 4.4.1. However, in section 4.4.2, we observed that the second statement was not satisfied. The high-momentum scaling occurred for $q>4.0 \mathrm{fm}^{-1} \approx 3 k_{F}$. This effect can be traced back to the definition of 2-body momentum distributions: the probability to find two nucleons of a given type at some relative momentum $(q)$ and some center-ofmass momentum $(Q)$. Care should be taken to identify SRCs in 2-body momentum distributions, since these distributions include any type of pair, whether they are physically correlated at short distances or not. As an example, consider a pair of nucleons, one of which has a momentum $k_{1}=3 k_{F}$ (and therefore belongs to the high-momentum correlated tail of the momentum distribution), and the other one is at rest: $k_{2}=0$ (and therefore belongs to the Mean-Field part of the momentum distribution). The relative momentum for these two uncorrelated nucleons is high $\left(q=\left|\vec{k}_{1}-\vec{k}_{2}\right| / 2=1.5 k_{F}\right)$. That is, this pair could be naively interpreted as a short-range correlated pair. Nevertheless, their center-of-mass momentum is also high $\left(Q=\left|\vec{k}_{1}+\vec{k}_{2}\right|=3 k_{F}\right)$, and SRCs have low $Q$ [50]. Therefore, the high- $q$ condition is necessary, but not sufficient. Additionally, we need to require either for the pair to have low center-of-mass momentum, or alternatively for each individual nucleon to have high individual momenta.

To study this idea beyond the simple example described in the previous paragraph, we developed a toy model that describes the nuclear environment as a Correlated Fermi Gas (CFG, not to be confused with the Generalized Contact Formalism, GCF). 
The CFG model describes the nucleus as a Fermi gas at zero temperature with a shortrange interaction between the fermions. That is, the 1-body momentum distribution corresponds to:

$$
n_{\mathrm{CFG}}(k)=\left\{\begin{array}{l}
C_{1}, \quad k<k_{F} \\
C_{2} / k^{n}, \quad k_{F} \leq k \leq 5 \mathrm{fm}^{-1} \\
0, \quad \text { otherwise, }
\end{array}\right.
$$

where $C_{1}$ and $C_{2}$ are normalization constants representing the strength of the MeanField part of the momentum distribution and the high-momentum, SRC dominated tail respectively. The Mean-Field part of the momentum distribution was defined with an $80 \%$ strength:

$$
\begin{aligned}
\int_{0}^{k_{F}} k^{2} n_{\mathrm{CFG}}(k) d k & =0.8 \\
C_{1} \int_{0}^{k_{F}} k^{2} d k & =\left.C_{1} \frac{k^{3}}{3}\right|_{0} ^{k_{F}}=0.8 \\
C_{1} & =0.8 \frac{3}{k_{F}^{3}}
\end{aligned}
$$

Since all the nucleons in the tail are expected to be correlated with another nucleon, we defined the strength of the tail to be $10 \%$, and every time a nucleon was sampled from this region of the distribution, another nucleon was created, completing the remaining $20 \%$ strength with respect to the Mean Field:

$$
\begin{aligned}
\int_{k_{F}}^{5 \mathrm{fm}^{-1}} k^{2} n_{\mathrm{CFG}}(k) d k & =0.1 \\
C_{2} \int_{k_{F}}^{5 \mathrm{fm}^{-1}} \frac{d k}{k^{n-2}} & =\left.C_{2} \frac{k^{3-n}}{3-n}\right|_{k_{F}} ^{5 \mathrm{fm}^{-1}}=0.1 \\
C_{2} & =0.1 \times \frac{3-n}{5^{3-n}-k_{F}^{3-n}} .
\end{aligned}
$$

We used $n=4$, following the arguments from [146], in which case: $C_{2}=0.1 /\left(\frac{1}{k_{F}}-\right.$ $\left.\frac{1}{5}\right)$. Many events were sampled from $k^{2} n_{\mathrm{CFG}}(k)$. Every time a nucleon with momentum $k_{1}>k_{F}$ was sampled, we also sampled three center-of-mass momenta values $Q$ (one for each cartesian coordinate) from three gaussian distributions with mean $\mu=0$ and 
standard deviation $\sigma=140 \mathrm{MeV} / c$, as measured by Cohen et al. [50]. Then, $k_{1}$ is turned into a vector by sampling a unit vector from a uniform sphere. Accordingly, we create another (correlated) nucleon with momentum:

$$
\vec{k}_{2}=\vec{Q}-\vec{k}_{1}
$$

The resulting 2-body momentum distribution is shown in Fig. 4-6 as a function of $q$ and $Q$ and is built by looping over every possible pair of nucleons, and classifying each pair according to the origin of the two nucleons:

- the two nucleons are from the Mean Field: MF-MF

- one nucleon is from the Mean Field, and the other one is from the highmomentum tail: MF-SRC

- the two nucleons are from the high-momentum tail, but they are not correlated with one another: SRC-SRC (not same pair)

- the two nucleons are from the high-momentum tail, and they belong to the same SRC pair: SRC-SRC (same pair)

As expected, true short-range correlated pairs (bottom-right plot) dominate the 2body momentum distribution at low- $Q$ and high- $q$ simultaneously, and uncorrelated MF-MF pairs (bottom-left plot) dominate the 2-body momentum distribution for $q<k_{F}$. The delayed scaling seen in the previous section comes from the uncorrelated MF-SRC pairs (top-right plot), which dominate the 2-body momentum distribution up to higher values of $q$ as more center-of-mass motion is incorporated. Section IV.A of [149] describes how SRCs dominate the 2-body momentum distribution for $q \gtrsim 1.0+0.5 \times Q$. This relation, which was studied using realistic 2 -body momentum distributions and is plotted in Fig. 4-6 as a diagonal yellow line in the MF-SRC (top-right) plot, agrees with the MF-SRC boundary from this simple model.

Thus there are two main ways to identify SRCs in 2-body momentum distributions while minimizing the Mean-Field contribution. We can either 1) restrict the study 


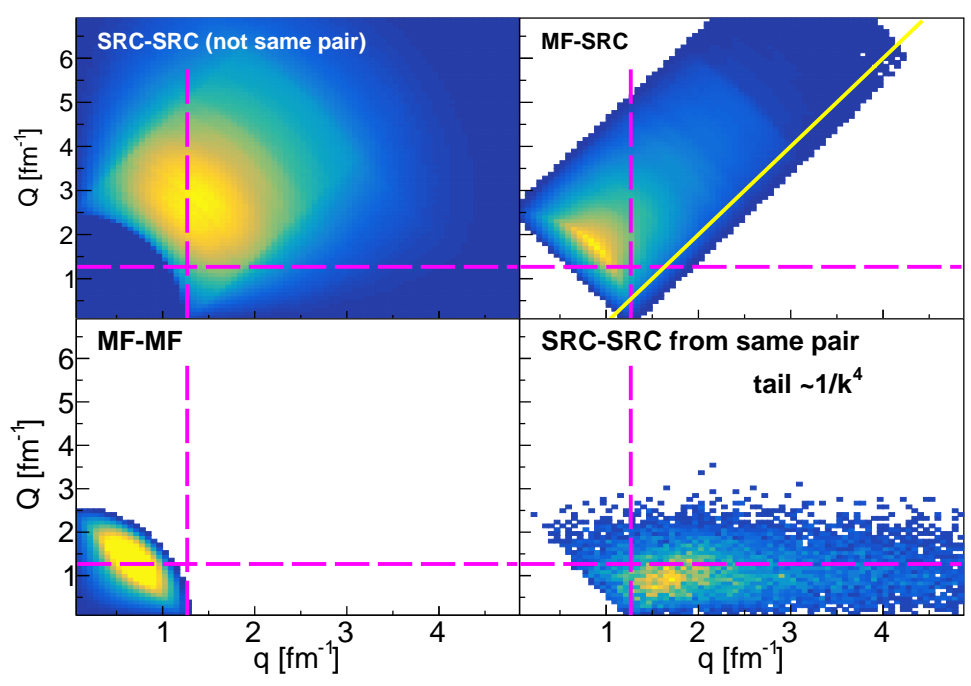

Figure 4-6: Toy model 2-body momentum distribution as a function of center-of-mass $(Q)$ and relative $(q)$ momenta corresponding to nucleons that follow equation 4.18 with $n=4$. The dashed magenta lines correspond to $k_{F}$. The diagonal yellow line shows, for a given $Q$, the minimum $q$ value above which SRC dominate the 2-body momentum distribution [149].

to low- $Q$ and then SRCs will dominate for $q \gtrsim k_{F}$, or 2) we can integrate over $Q$, and then SRCs will dominate for $q \gg k_{F}$. These two approaches can be further seen by comparing experimental data to ab-initio calculations. Fig. 4-7 shows the ${ }^{4} \mathrm{He}$ SRC $p p / p n$ ratio as a function of $q$ adapted from Fig. 1 in [138]. The magenta markers correspond to the SRC pair fraction extracted from electron-induced protonnucleon knockout ${ }^{4} \mathrm{He}\left(e, e^{\prime} p N\right)$ measurements [56]. The kinematics of the experiment are chosen to detect SRC pairs. The colored lines correspond to the ratio:

$$
\frac{\mathrm{SRC}_{p p}}{\mathrm{SRC}_{p n}} \equiv \frac{\int_{0}^{K_{\max }} d \vec{Q} F_{p p}(\vec{q}, \vec{Q})}{\int_{0}^{K_{\max }} d \vec{Q} F_{p n}(\vec{q}, \vec{Q})},
$$

where different colors correspond to different values of $K_{\max }$ varying from 0 to $\infty$. As long as the integral upper limit is small $\left(K_{\max } \lesssim k_{F}\right)$, calculated ratios agree with the measured data. For integrations carried out up to $K_{\max } \geq 2 \mathrm{fm}^{-1}$, the calculations only agree with the measured data for $q \gg k_{F}$.

The 2-body momentum distributions used in section 4.4.2 to extract the nuclear contacts in momentum space correspond to distributions integrated over center-of- 


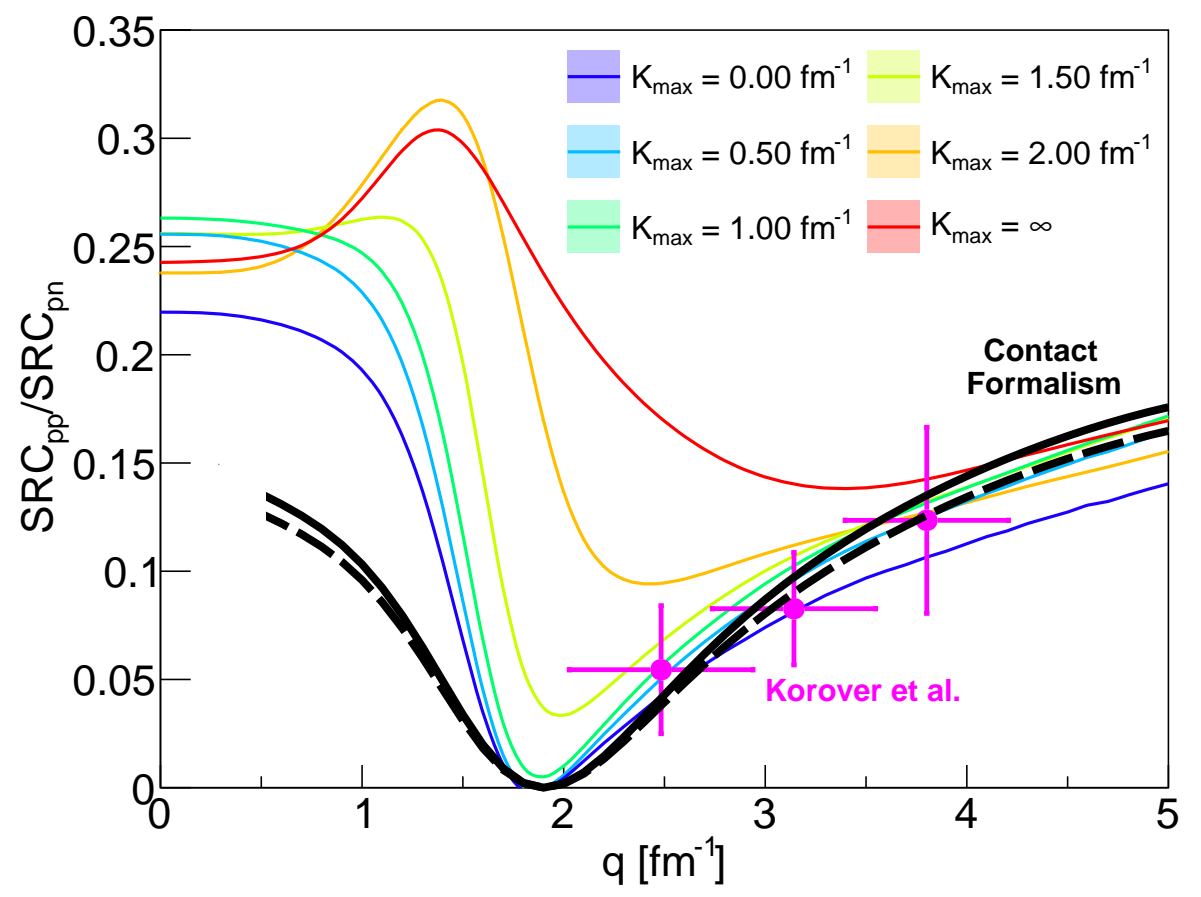

Figure 4-7: ${ }^{4} \mathrm{He}$ SRC $p p / p n$ fraction as a function of $q$ adapted from Fig. 1 in [138. The magenta markers correspond to the SRC pair fraction extracted from ${ }^{4} \mathrm{He}\left(e, e^{\prime} p N\right)$ measurements [56]. The colored lines correspond to AV18+UX VMC 2-body momentum distributions integrated over center-of-mass momentum in the range $0 \leq Q \leq K_{\max }$ (where $K_{\max }$ is specified in the legend). The solid and dashed black lines correspond to the prediction from the GCF calculated using the contacts extracted in momentum and coordinate space respectively. 
mass momentum: $F(\vec{q})=\int_{0}^{\infty} d \vec{Q} F(\vec{q}, \vec{Q})$. This explains why the expected scaling was delayed up to $q \approx 4.0 \mathrm{fm}^{-1}$.

Fig. 4-7 also shows the GCF prediction using the ${ }^{4} \mathrm{He}$ contacts from Table 4.1. In the GCF, this observable is calculated as:

$$
\frac{\mathrm{SRC}_{p p}}{\mathrm{SRC}_{p n}}(q)=\frac{C_{p p}^{s=0}\left|\varphi_{p p}^{s=0}(q)\right|^{2}}{C_{p n}^{s=0}\left|\varphi_{p n}^{s=0}(q)\right|^{2}+C_{p n}^{s=1}\left|\varphi_{p n}^{s=1}(q)\right|^{2}}
$$

The momentum- and coordinate-space contact results agree with each other and with both the measurement and the low- $Q$ VMC calculations. The same agreement is seen among GCF, VMC and data for ${ }^{12} \mathrm{C}$ [57].

\subsubsection{Contact extraction from experimental data}

We used the ${ }^{4} \mathrm{He}$ and ${ }^{12} \mathrm{C}\left(e, e^{\prime} p N\right)$ data [56, 57] to evaluate the GCF results. Conversely, we also used the $p p / p n$ ratios from this data, together with $a_{2}(A)$ scale factors, to directly extract contact values. $a_{2}(A)$ is interpreted as the number of SRC pairs in a given nucleus $A$ relative to deuterium, see section 1.3 . This parameter can be related to the GCF through the expression:

$$
a_{2}(A) \int_{k_{F}}^{\infty}\left|\psi_{d}(\vec{k})\right|^{2} d \vec{k}=\frac{C_{n n}^{s=0}+C_{p p}^{s=0}+C_{p n}^{s=0}+C_{p n}^{s=1}}{A / 2}
$$

where $\psi_{d}(\vec{k})$ is the total deuteron wave function normalized such that: $\int_{0}^{\infty}\left|\psi_{d}(\vec{k})\right|^{2} d \vec{k}=$ 1. We simultaneously fitted equations 4.27 and 4.28 assuming isospin symmetry to the available data. The results are presented in Table 4.1 with the text "(exp)" next to them.

\subsubsection{AV18 results and discussion}

We extracted the nuclear contacts following the three procedures described in the previous sections: 1) extraction in coordinate space from VMC 2-body coordinate densities, 2) extraction in momentum space from VMC 2-body momentum distributions, and 3) extraction in momentum space from experimental data. All three used 
the AV18 NN potential. The results are presented in Table 4.1 as the percentage of nucleons above $k_{F}$ in the different SRC channels.

These results show that some important features of SRCs are naturally obtained in the GCF. First and foremost, $n p$ dominance is manifested in that the $C_{p n}^{s=1}$ contacts are an order of magnitude greater than their spin-0 counterparts. Secondly, the agreement between contacts extracted in momentum and coordinate spaces from VMC calculations indicates a quantitative agreement between short-distance and high-momentum scaling of SRC pairs in nuclei.

More experimental data is needed to constrain the experimental contacts to a greater precision. Nevertheless, with the currently available data, we found that these contacts approximately agree with the corresponding contacts extracted from VMC 2-body densities.

A surprising result of the GCF concerns isospin symmetry in symmetric nuclei. While we expected to see a combinatorial relation between different pairs (i.e. $p p$, $p n, n n)$, we observed that all the spin-0 contacts are the same within uncertainties.

\subsubsection{Contact verification with 1-body momentum distribu- tions}

Up to this point, I described how we determined nuclear contacts from fits to 2-body coordinate densities and momentum distributions. That is, the 1-body momentum distribution from equation 4.14 has not been used and, consequently, it can now be used to verify the results. The left side of Fig. 4-8 shows a comparison between the VMC and the GCF proton 1-body momentum distributions for ${ }^{4} \mathrm{He}$, both based on the AV18 NN potential. The contributions from different contact channels are shown as dashed lines. The right side of Fig. 4-8 shows a similar comparison between VMC and the GCF proton 1-body momentum distributions for several symmetric nuclei. See Appendix $\mathrm{D}$ for plots equivalent to the left panel for the remaining nuclei.

It is worth reminding the reader that, since 2-body momentum distributions contain information from non-SRC "pairs" (see section 4.4.2), the contacts are determined 


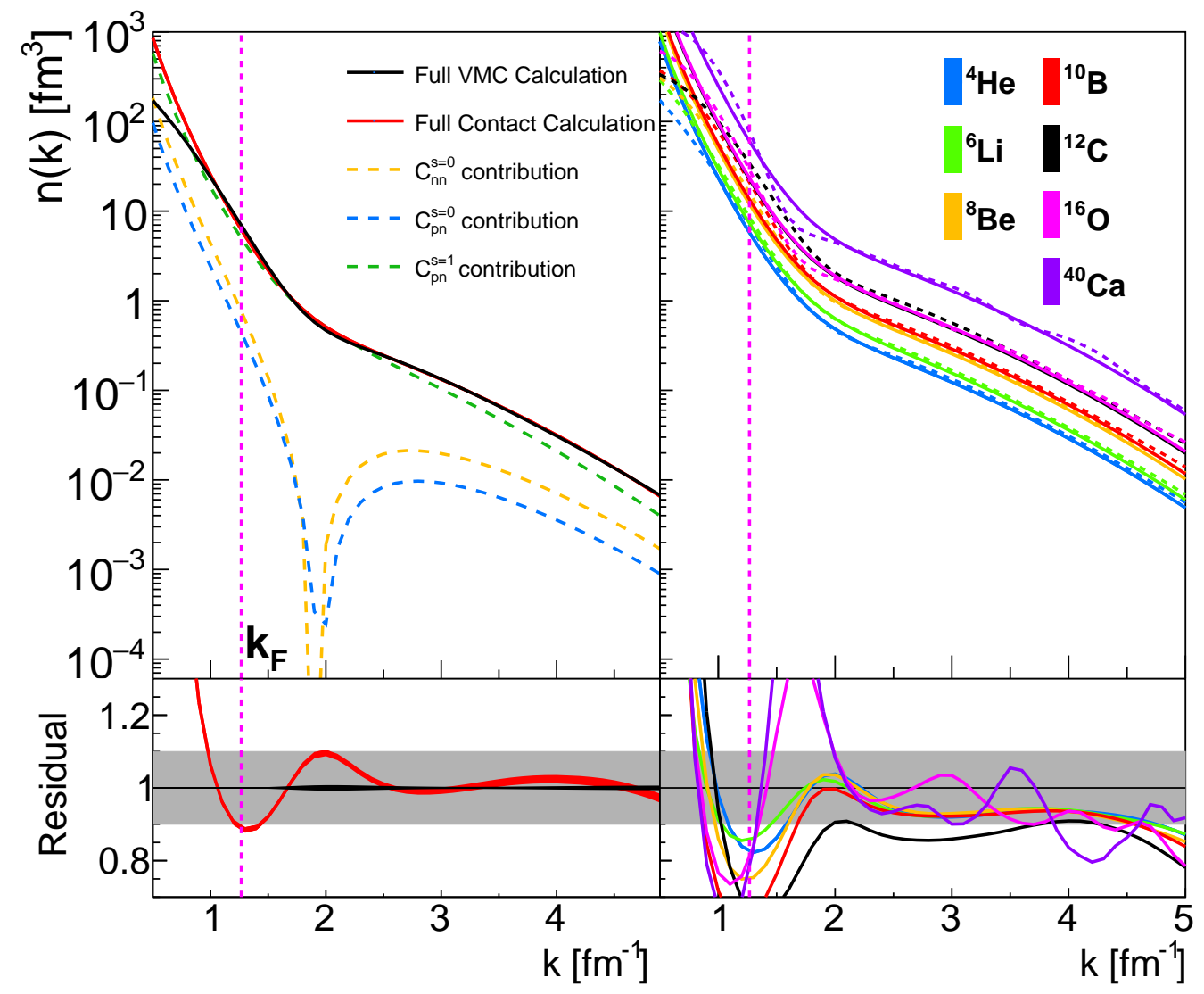

Figure 4-8: Comparison between 1-body momentum distributions from VMC and from the GCF. The left panel shows the comparison for ${ }^{4} \mathrm{He}$ and also the GCF prediction for the contribution from different types of pairs. The sum of the dashed lines equals the solid red line. The lower panel shows the ratio of the GCF over the VMC 1-body momentum distributions. The gray band shows the $10 \%$ level of agreement. The width of the red band corresponds to the uncertainty from the contacts, and the width of the black band of value 1 corresponds to the uncertainty from the VMC calculation. The right panel shows the comparison for different symmetric nuclei. 
from fits in the high-momentum range $4-5 \mathrm{fm}^{-1}$. The GCF describes the VMC 1body momentum distributions remarkably well, within $10-20 \%$ over three orders of magnitude.

\subsubsection{SRCs and the $S T=11$ channel}

We find a negligible SRC contribution from the spin-isospin $S T=11$ channel. Despite completely neglecting this channel we can reproduce the 1-body momentum distribution to within $10-20 \%$. However, previous work (e.g. Feldmeier et al. [150], and Alvioli et al. [151]) found a significant $S T=11$ contribution. A possible explanation for this discrepancy is that of section 4.4.2. That is, these two studies do not limit the pair center-of-mass momentum to be small, potentially leading to the inclusion of non-correlated pairs into their SRC studies.

\subsection{Testing scale-and-scheme independence with the GCF}

In the previous sections, I outlined how we developed and explored the validity of the GCF in the context of the AV18 potential. As described in section 1.2, and shown in Fig. 4-9, ab initio many-body calculations carried out with different $N N$-interaction models produce nuclear wave functions that differ significantly at short distances and high momenta. This scale and scheme dependence raises important questions about the model dependence of SRC measurement interpretations.

In order to address this issue, Chen et al. [152] and Lynn et al. [142] analyzed QMC calculations of 2-body coordinate densities calculated from different realistic $N N$-interaction models (without separating the contributions from different spinisospin channels) and showed the first evidence for scale and scheme independence of ratios of 2-body coordinate densities for different nuclei to the deuteron at short distances.

Here, I present a comprehensive study of the scale and scheme (in)dependence 

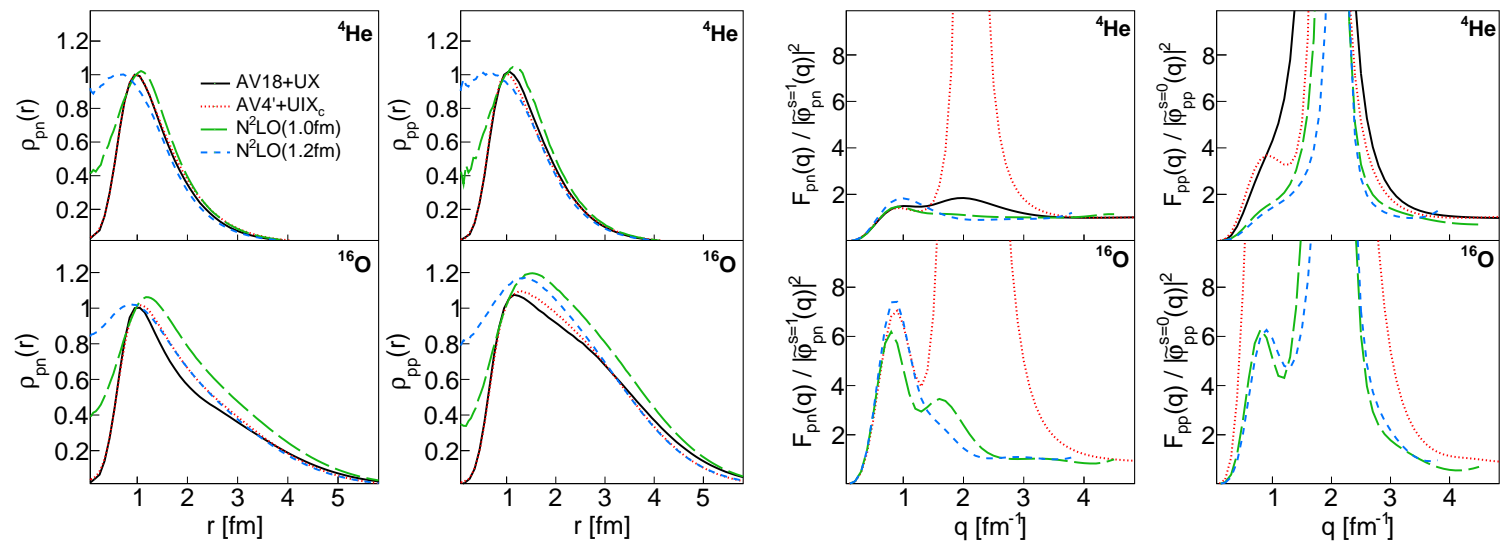

Figure 4-9: 2-body coordinate densities (left two columns) and momentum distributions (right two columns) calculated using different $N N$ potential models. The top and bottom rows correspond to ${ }^{4} \mathrm{He}$ and ${ }^{16} \mathrm{O}$ respectively.

of different SRC properties from analyzing both coordinate densities and momentum distributions (projected into spin-isospin channels) calculated from different realistic $N N$-interaction models using a common framework: the GCF. Specifically, I will focus on the four interactions introduced and described in section 1.1: the phenomenological AV18, AV4', and the chiral $\mathrm{N}^{2} \mathrm{LO}(1.0 \mathrm{fm}), \mathrm{N}^{2} \mathrm{LO}(1.2 \mathrm{fm})$, and NV2+3-Ia* interactions.

As described in section 4.3, the first step in the GCF corresponds to determining the universal $N N$ asymptotic wave functions, corresponding to the zero-energy numerical solution of the Schrödinger equation, which depends on a specific $N N$ potential. That is, $\varphi_{N N}^{\alpha}$ are universal in the weak sense (they are nucleus but not model independent). Consequently, a set of $N N$ asymptotic wave functions needs to be calculated for each $N N$ potential being studied. In Fig. 4-10, we can see the scale and scheme dependence of the $N N$ asymptotic densities.

We begin checking the validity of the GCF by studying the short-distance and high-momentum universality of 2-body coordinate densities and momentum distributions respectively, calculated with different $N N$-interaction models as was done in section 4.4 for the AV18 potential. The left column of Fig. 4-11 shows the 2-body coordinate densities for all four interactions and different nuclei, normalized to have the same value at $\sim 1 \mathrm{fm}$. While the short-distance behavior is very different among $N N$ models, for each model all nuclei exhibit the same behavior. Furthermore, this 

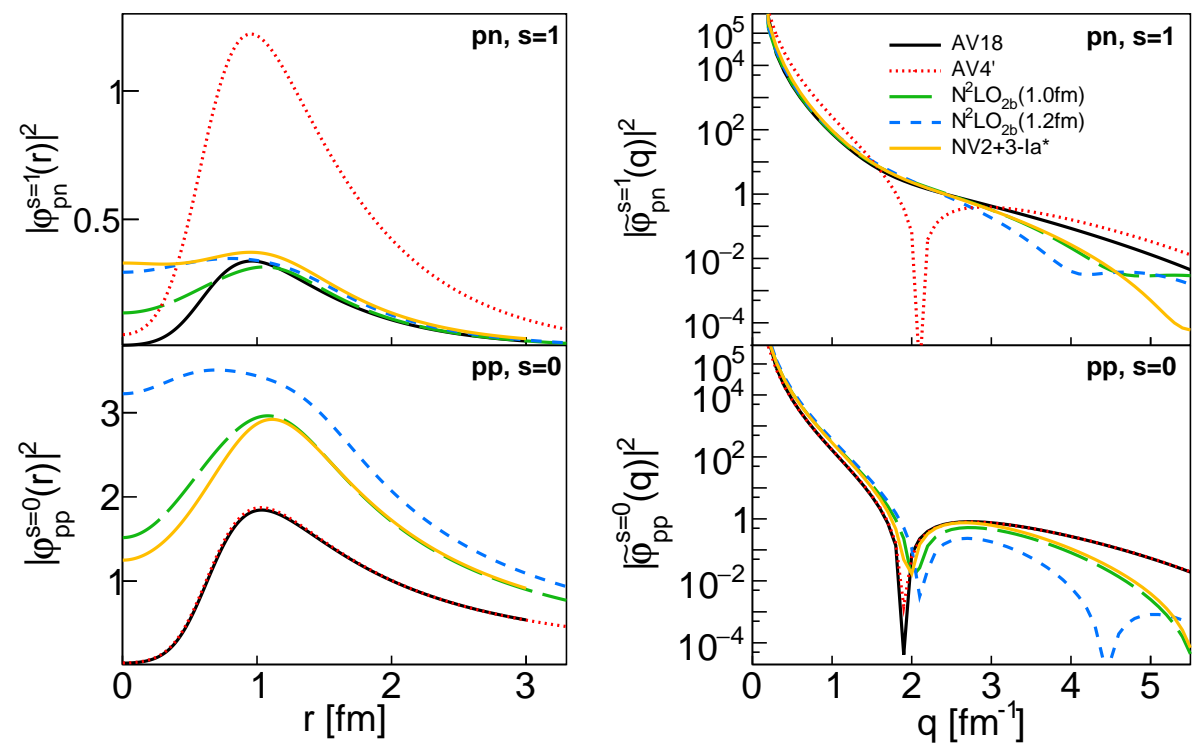

Figure 4-10: Universal asymptotic densities $\left|\varphi_{N N}^{\alpha}\right|^{2}$ for spin-1 pn (top) and spin-0 pp (bottom) pairs calculated in both coordinate (left) and momentum (right) space for five different $N N$ potentials. The spin-0 $n n$ and $p n$ functions are quantitatively very similar to the spin-0 $p p$ functions. Here, $\vec{r}=\vec{r}_{i j}$ is the relative distance between the nucleons in the pair, $\vec{q}=\left(\vec{k}_{i}-\vec{k}_{j}\right) / 2$ is the relative momentum between the nucleons in the pair, and the $i j$ indices have been dropped.

behavior is consistent with that of the universal asymptotic densities (shown in black). This validates equation 4.10 for the different interactions at short-distances. Since the 2-body momentum distributions decay exponentially, the right column of Fig. 4-11 shows the 2-body momentum distributions divided by the corresponding universal asymptotic density, scaled to a value of one at high momenta $\left(4.5 \mathrm{fm}^{-1}\right.$ in the case of phenomenological potentials, and $3.5 \mathrm{fm}^{-1}$ in the case of chiral interactions). As expected from equation 4.11, we found that the high-momentum part of the resulting distributions is constant. For $p p$ and $n n$ pairs, the scaling is less pronounced and starts at higher momenta than that for pn pairs, possibly due to three-nucleon SRCs, but is still present. As discussed previously in section 4.4.2, the 2-body momentum distribution scaling onset is much higher than expected due to the presence of uncorrelated pairs.

The toy model from section 4.4 .2 suggests that the scaling onset also depends on the "hardness" of the interaction, with phenomenological potentials scaling at 

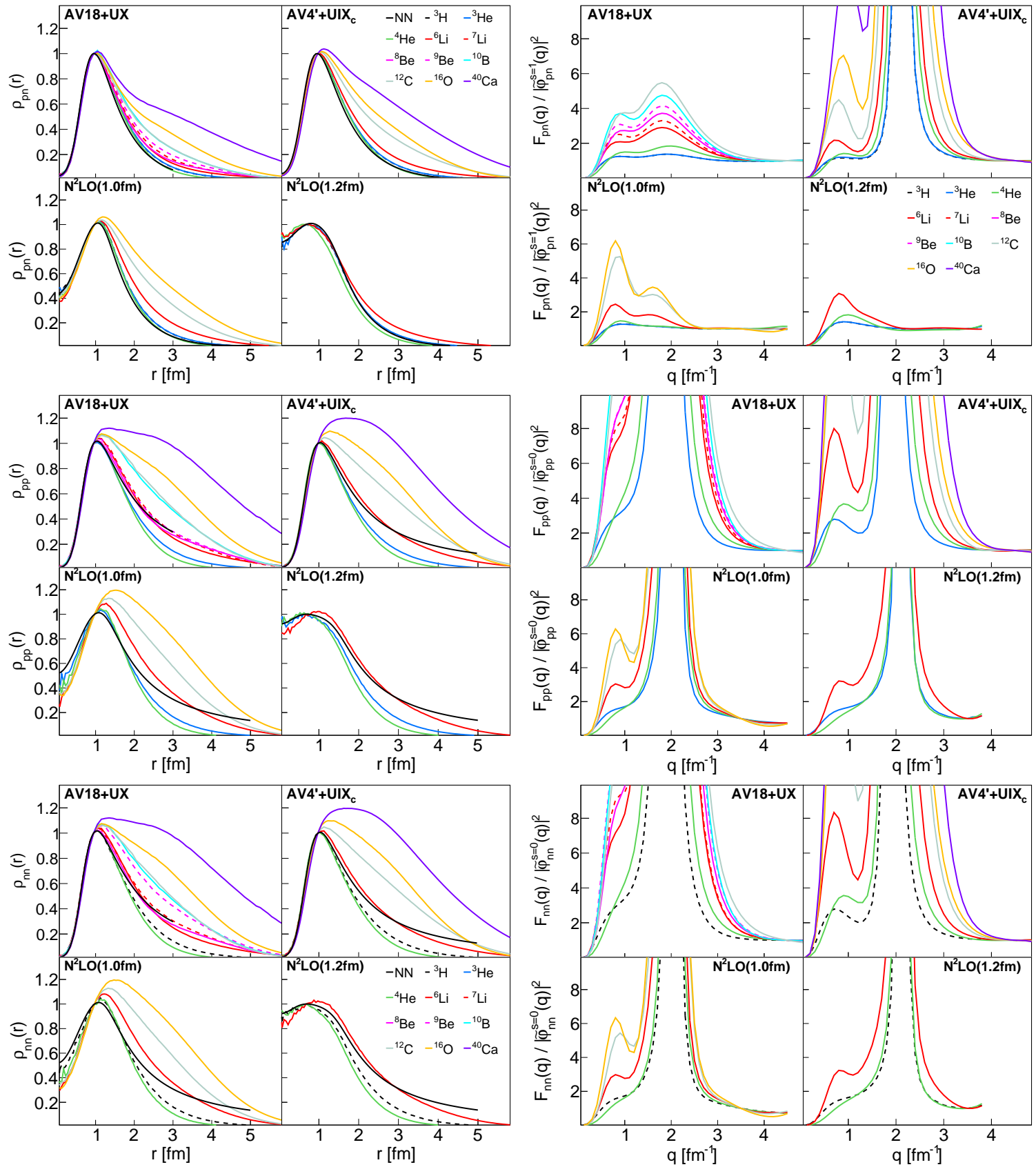

Figure 4-11: Short-distance (left column) and high-momentum (right column) universality of 2-body coordinate densities and momentum distributions respectively for $p n$ (top), $p p$ (center), and $n n$ (bottom) pairs for different $N N+3 N$ interactions. The $\mathrm{N}^{2} \mathrm{LO}(1.0 \mathrm{fm})$ and $(1.2 \mathrm{fm})$ distributions are only shown up to 4.4 and $3.8 \mathrm{fm}^{-1}$ respectively, above which cutoff effects become very large. 
higher momenta. This is confirmed by the distributions from Fig. 4-11. For example, in the case of $p n$ pairs, we can see that the scaling starts at $3.4-4 \mathrm{fm}^{-1}$ for the phenomenological, and at $2-2.3 \mathrm{fm}^{-1}$ for the chiral potentials.

Now that the GCF has been validated for the considered $N N$ interactions, we can proceed to study the scale and scheme (in)dependence of different SRC properties.

\subsubsection{Scale and scheme independence of nuclear contacts and position-momentum equivalence of SRCs}

The GCF 2-body coordinate densities (equation 4.10) and momentum distributions (equation 4.11) include the contribution from the 2-body universal functions $\left(\varphi_{N N}^{\alpha}\right.$, which describe the short-distance and high-momentum pair interaction), and the nuclear contacts $\left(C_{N N}^{\alpha}\right.$, which encode the information related to the many-body dynamics that drive the formation and abundance of SRCs). As shown in Fig. 4-10, the 2-body universal functions are largely scale and scheme dependent. To study the scale and scheme dependence of nuclear contacts independently from the universal functions, we examined ratios of 2-body coordinate densities and momentum distributions at short distances and high momenta respectively, in nucleus $A$ relative to a reference nucleus $A_{0}$. We used the smallest symmetric reference nucleus for each channel: $\mathrm{d}$ for $p n, s=1$, and ${ }^{4} \mathrm{He}$ for the other channels. Thus, according to equation 4.10 and 4.11 the universal functions cancel in the ratio and, consequently, this result corresponds to the ratio of contacts for $A$ to $A_{0}$ :

$$
\frac{\left.\rho_{N N}^{\alpha}(\vec{r})\right|_{A}}{\left.\rho_{N N}^{\alpha}(\vec{r})\right|_{A_{0}}}=\frac{\left.\left|\varphi_{N N}^{\alpha}(\vec{r})\right|^{2} C_{N N}^{\alpha}\right|_{A}}{\left.\left|\varphi_{N N}^{\alpha}(\vec{r})\right|^{2} C_{N N}^{\alpha}\right|_{A_{0}}}=\frac{\left.C_{N N}^{\alpha}\right|_{A}}{\left.C_{N N}^{\alpha}\right|_{A_{0}}},
$$

and similarly in momentum space.

Fig. 4-12 shows the ratios $\left.C_{p n}^{s=1}\right|_{A} /\left.C_{p n}^{s=1}\right|_{d}$ and $\left.C_{N N}^{\alpha}\right|_{A} /\left.C_{N N}^{\alpha}\right|^{4} \mathrm{He}$ for all available nuclei and interactions. All contact ratios for a given nucleus are largely scale and scheme independent within uncertainties. The fact that $N N$-interaction models with very different short-distance structures, including the tensor-less AV4', all lead to the same nuclear contact ratios, implies that the SRC pair formation and abundances are 


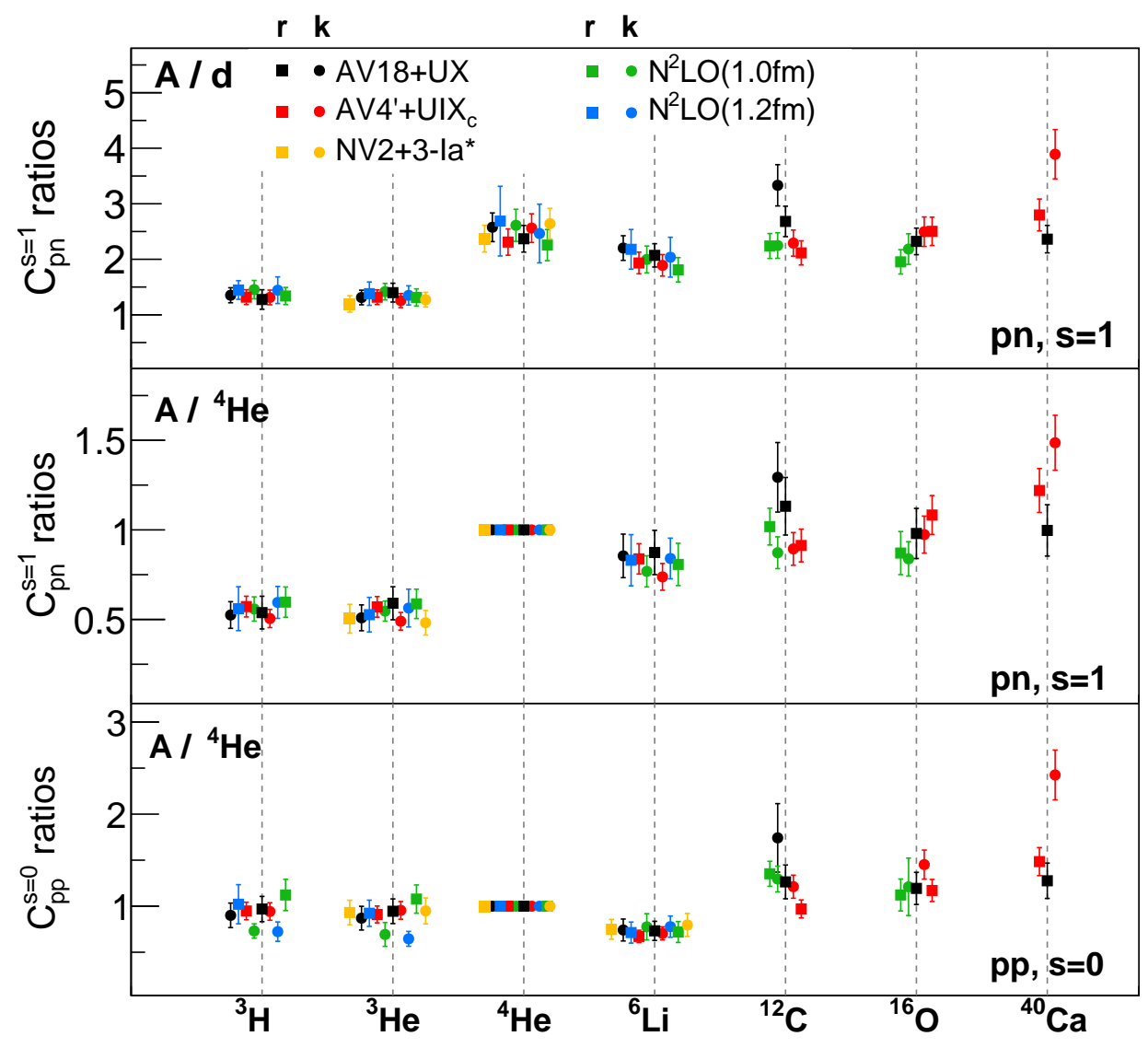

Figure 4-12: Ratios of $p n$ spin-1 nuclear contacts to deuterium (top), ${ }^{4} \mathrm{He}$ (center), and of $p p$ spin- 0 nuclear contacts to ${ }^{4} \mathrm{He}$ (bottom) for different nuclei, extracted for the available potentials in coordinate $(\boldsymbol{\square})$ and momentum (o) space. In the case of ${ }^{3} \mathrm{H}$, there are no $p p$ pairs, and the contact ratios shown for this nucleus in the bottom panel correspond to $C_{n n}^{s=0}$. The contacts have been divided by $A / 2$ and multiplied by 100 to give the percent of nucleons above $k_{F}$ in the different SRC channels. 
a property of the Mean Field (long-range physics) part of the $N N$ interaction, where all these $N N$ models agree. Our results are in agreement with those from Vanhalst [55], who found that the SRC abundances are a Mean-Field property, with only SRC properties such as the isospin structure and relative momentum distributions being determined by the $N N$ interaction structure at short distances.

QMC calculations with soft $N N$-interaction models are less computationally demanding than those with hard ones. We can use the scale and scheme independence of nuclear contact ratios in order to estimate contacts of heavier nuclei without needing their QMC calculations. For instance, let us imagine that, while both $\mathrm{N}^{2} \mathrm{LO}$ and AV18 QMC calculations exist for a reference nucleus $A_{0}$, only those carried out with the $\mathrm{N}^{2} \mathrm{LO}$ interaction exist for a heavier nucleus $A$. We can then estimate the AV18 contacts for $A$ as:

$$
\left.C_{N N}^{\alpha}\right|_{A, \mathrm{AV} 18}=\left.\left(\frac{\left.C_{N N}^{\alpha}\right|_{A}}{\left.C_{N N}^{\alpha}\right|_{A_{0}}}\right)\right|_{\mathrm{N}^{2} \mathrm{LO}} \times\left. C_{N N}^{\alpha}\right|_{A_{0}, \mathrm{AV} 18}
$$

These findings also have implications for experimental studies. It has been claimed in the literature that $a_{2}(A)$ (see sections 1.3 and 4.4.3) is sensitive to the nuclear interaction at short distances. This seemingly stands in contrast with the results of Chen et al. [152] and Lynn et al. [142], who found that ratios of 2-body coordinate densities (for all types of $N N$ pairs) for nucleus $A$ relative to deuterium are insensitive to the $N N$ interaction, and the numerical value for these ratios is consistent with the experimental $a_{2}(A)$ values for the studied nuclei. We strengthen these results by showing the scale and scheme independence of nuclear contact ratios in both coordinate and momentum space, and also for pairs with different quantum numbers. However, unlike in individual $N N, \alpha$ QMC distribution ratios, when all the possible $N N$ channels are included in an $a_{2}(A)$ calculation, the scale-and-scheme-dependent pair densities don't cancel. A rigorous derivation connecting $a_{2}(A)$ (defined experimentally) to QMC calculations and nuclear contacts is underway [153].

Exclusive two-nucleon knockout $A\left(e, e^{\prime} N N\right)$ measurements are sensitive to the nuclear interaction models. Fig. 4-13 shows $p p$-to-pn pair ratios from experiment and 
theory for ${ }^{4} \mathrm{He}$ as a function of relative momentum $q$. The experimental points are the same shown in Fig. 4-7 and correspond to $\left(e, e^{\prime} p p\right)$ and $\left(e, e^{\prime} p n\right)$ measurements from Korover et al. [56]. Some $N N$ interactions agree with the experimental ratios better than others, with the tensor-less AV4' interaction failing completely.

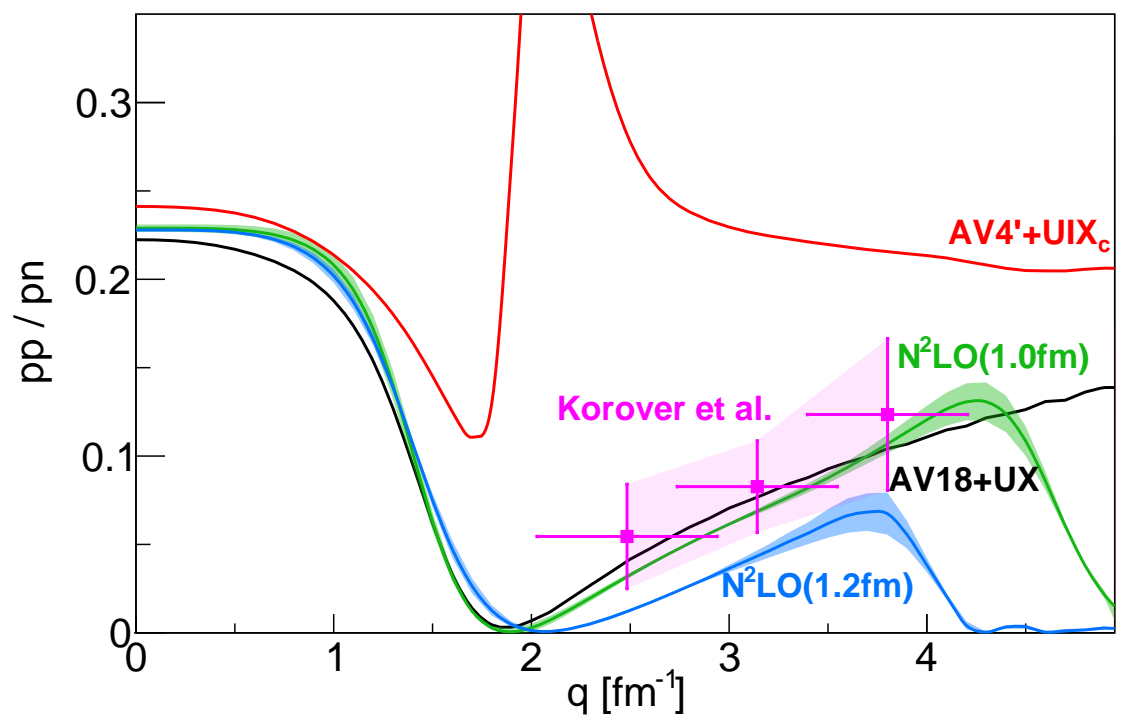

Figure 4-13: Ratios of ${ }^{4}$ He $p p$-to- $p n$ back-to-back $(Q=0)$ pairs from experiment [56] and theory as a function of relative momentum $q$. The lines correspond to ratios of 2body momentum distributions $F_{p p}(q, Q=0) / F_{p n}(q, Q=0)$ calculated using different $N N$ potentials.

Finally, there have been claims that the scaling of SRC pairs with high relative momentum is different from that of SRC pairs with small separation [154]. The agreement found between nuclear contact ratios extracted in coordinate and momentum spaces indicates that such claims are inconsistent with the QMC wave functions.

\subsubsection{Absolute contacts}

In the previous section, I described our findings from studying ratios of 2-body coordinate densities and momentum distributions at short distances and high momenta respectively. By taking such ratios, the scale-and-scheme-dependent asymptotic $N N$ densities cancel, allowing us to study the nuclear contact ratios. These ratios are scale and scheme independent for all the considered nuclei and $N N$ potentials. Having done so, we then proceeded to extract the individual contacts. 
The contact extraction process in the case of the AV18 distributions was described in section 4.4. Similarly, the contacts corresponding to the remaining $N N+3 N$ models were determined by fitting the VMC 2-body coordinate densities and momentum distributions to equations 4.10 and 4.11 respectively. Since not all the spin and isospin projections are available, we make some approximations:

- $C_{p p}^{s=0}$ and $C_{n n}^{s=0}$ are extracted from fits to the total $p p$ and $n n$ densities respectively, assuming that the dominant contribution comes from spin-0 (s-wave) pairs.

- $C_{p n}^{s=0}$ and $C_{p n}^{s=1}$ are extracted from fits to the isospin $\mathrm{T}=1$ and $\mathrm{T}=0$ pn distributions respectively, assuming that the dominant contribution comes from spin-0 and spin-1 pn pairs.

Appendix E shows examples of these fits in the case of ${ }^{4} \mathrm{He}$.

Fig. 4-14 shows the absolute contacts extracted in coordinate and momentum space for all available nuclei and $N N+3 N$ interactions. The top and bottom panels show the $p n$ spin-1 and $p p$ spin-0 contacts respectively. These values are also presented in Table 4.2. For symmetric nuclei, $C_{n n}^{s=0}=C_{p p}^{s=0}$. For ${ }^{3} \mathrm{He}, C_{n n}^{s=0}=0$. For ${ }^{3} \mathrm{H}$, $C_{p p}^{s=0}=0$, and the value shown in this columns corresponds to $C_{n n}^{s=0}$.

The $p n$ spin-1 contacts are largely scale and scheme independent for all interactions in all channels, except for those determined from AV4' distributions. The abundance of pn spin-1 SRC pairs are predicted to be much less in this case, since the AV4' interaction does not have the tensor force responsible for $p n$ dominance.

The $p p$ spin- 0 contact results are more complicated. The AV18 and AV4' contacts (both in coordinate and momentum space) and the $\mathrm{N}^{2} \mathrm{LO}$ momentum-space contacts agree overall with each other, but are higher than the NV2+3-Ia* (both in coordinate and momentum space) and the $\mathrm{N}^{2} \mathrm{LO}$ coordinate-space contacts. This behavior is less well understood than the spin-1 case from the previous paragraph. To further illustrate this, Fig. 4-15 shows ratios of $r$ - to $k$-space $p n$ spin-1 (top) and $p p$ spin-0 (bottom) nuclear contacts for all the available nuclei and interactions. Again, these results confirm that the short-distance and high-momentum scaling of 2-body 


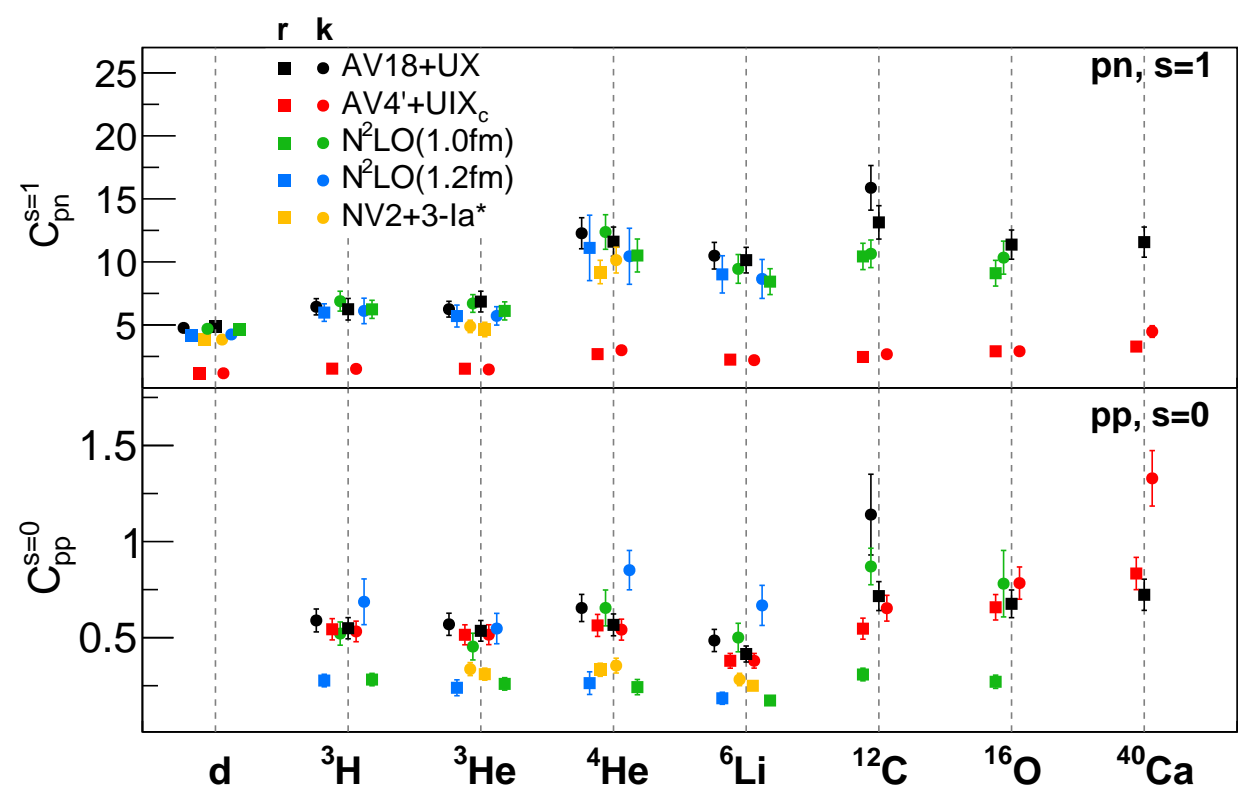

Figure 4-14: Absolute values of the $p n$ spin-1 (top) and $p p$ spin- 0 contacts extracted in coordinate (-) and momentum (-) space from QMC calculations using different $N N$-interaction models. In the case of ${ }^{3} \mathrm{H}$, there are no $p p$ pairs, and the contacts shown for this nucleus in the bottom panel correspond to $C_{n n}^{s=0}$. The contacts have been divided by $A / 2$ and multiplied by 100 to give the percent of nucleons above $k_{F}$ in the different SRC channels.

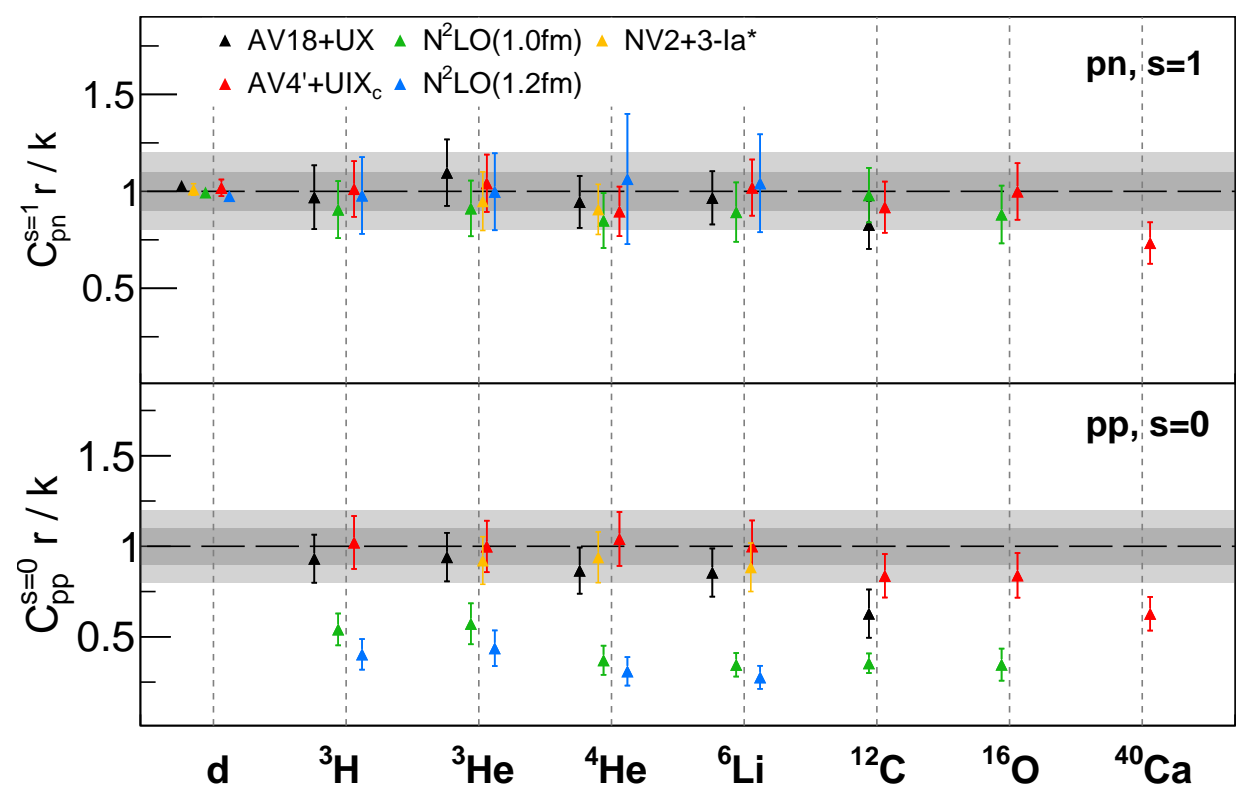

Figure 4-15: Ratios of coordinate- to momentum-space nuclear contacts for $p n$ spin-1 (top) and $p p$ spin-0 (bottom) pairs for different nuclei. In the case of ${ }^{3} \mathrm{H}$, there are no $p p$ pairs, and the contacts shown for this nucleus in the bottom panel correspond to $C_{n n}^{s=0}$. The dark- and light-gray bands correspond to the $10 \%$ and $20 \%$ levels of agreement respectively. 


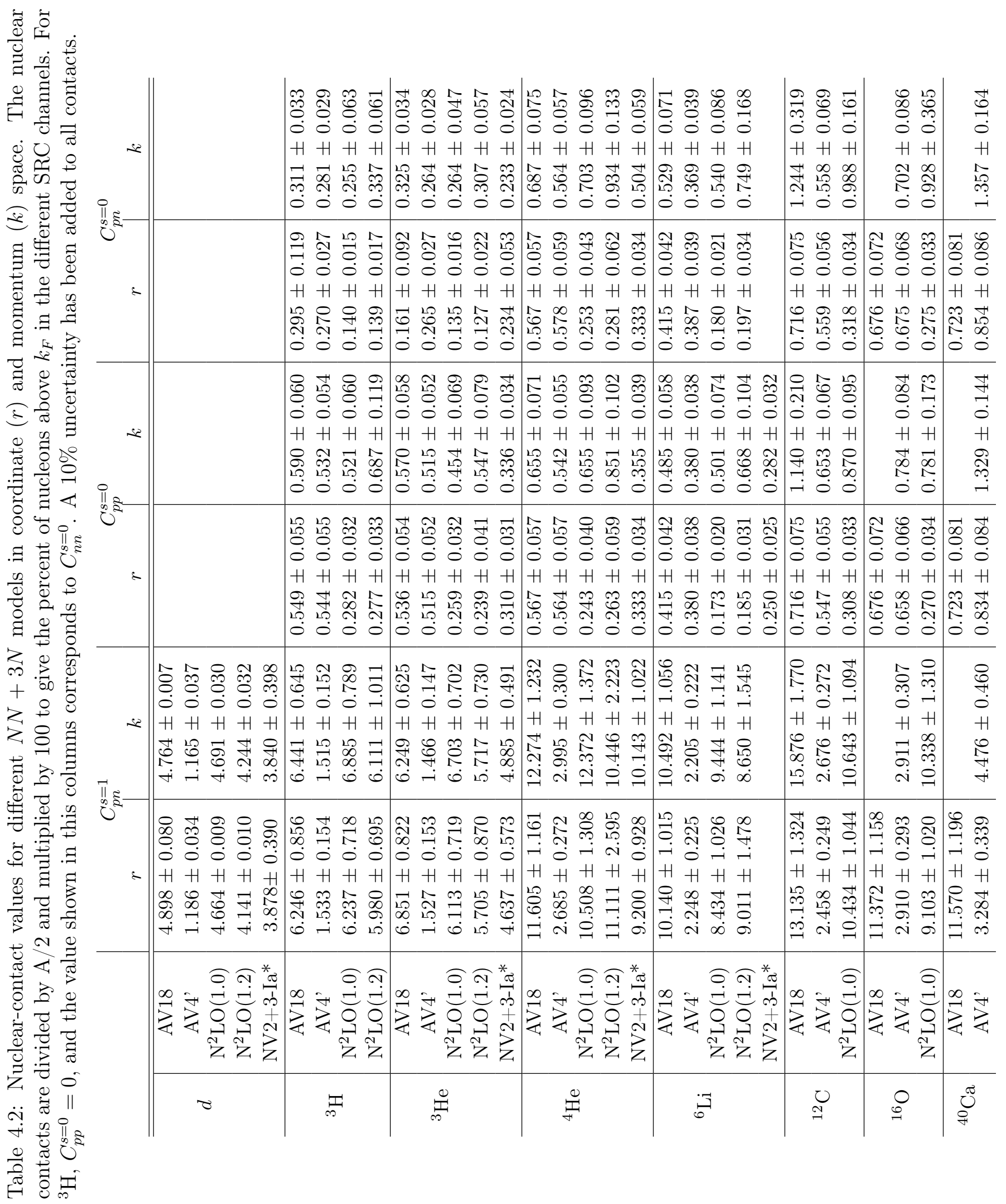


coordinate densities and momentum distributions are consistent with each other for all available nuclei, channels, and $N N$-interaction models, except for the case of the $\mathrm{N}^{2} \mathrm{LO}$ spin-0 channel. The fact that this coordinate-momentum scaling discrepancy only happens in the case of $\mathrm{N}^{2} \mathrm{LO}$ and not with $\mathrm{NV} 2+3-\mathrm{Ia}^{*}$, while both are chiral interactions with short-distance cutoffs, implies that the disagreement is not an inherent

characteristic of chiral interactions, but a feature of the $\mathrm{N}^{2} \mathrm{LO}$ potential, presumably coming from its lower order in the chiral expansion and lack of intermediate deltas.

\subsubsection{Systematic uncertainties}

We included three main sources of contact and contact-ratio uncertainties:

1. Parameter sensitivity: as it was done in section 4.4, the systematic uncertainty of the contacts and contact ratios extracted in this section are determined from a sensitivity study. That is, we vary the fit range within reasonable limits and take the resulting contact spread as the uncertainty. See Appendix Efor details.

2. Calculational precision: all the QMC calculations for different nuclei and $N N$ potentials used in this chapter are available for VMC. Therefore, for consistency, we extracted all the contacts and contact ratios using VMC 2-body coordinate densities and momentum distributions. Additionally, $\mathrm{N}^{2} \mathrm{LO}(1.0 \mathrm{fm})$, $\mathrm{N}^{2} \mathrm{LO}(1.2 \mathrm{fm})$, and AV4' 2-body coordinate densities are now available for some nuclei using DMC and EXT (see section 1.2 for details). Fig. 4-16 shows ratios of nuclear contacts extracted from VMC 2-body coordinate densities to nuclear contacts extracted from DMC and EXT 2-body coordinate densities. Based on these results, we added an uncertainty conservatively fixed at $10 \%$ to all the contacts and contact ratios as a measure of the QMC calculational error.

3. $3 N$-interaction uncertainty: when calculations are available with different $3 N$ force models ( $\mathrm{N}^{2} \mathrm{LO}$ potentials), we include the difference in the results as the uncertainty. 


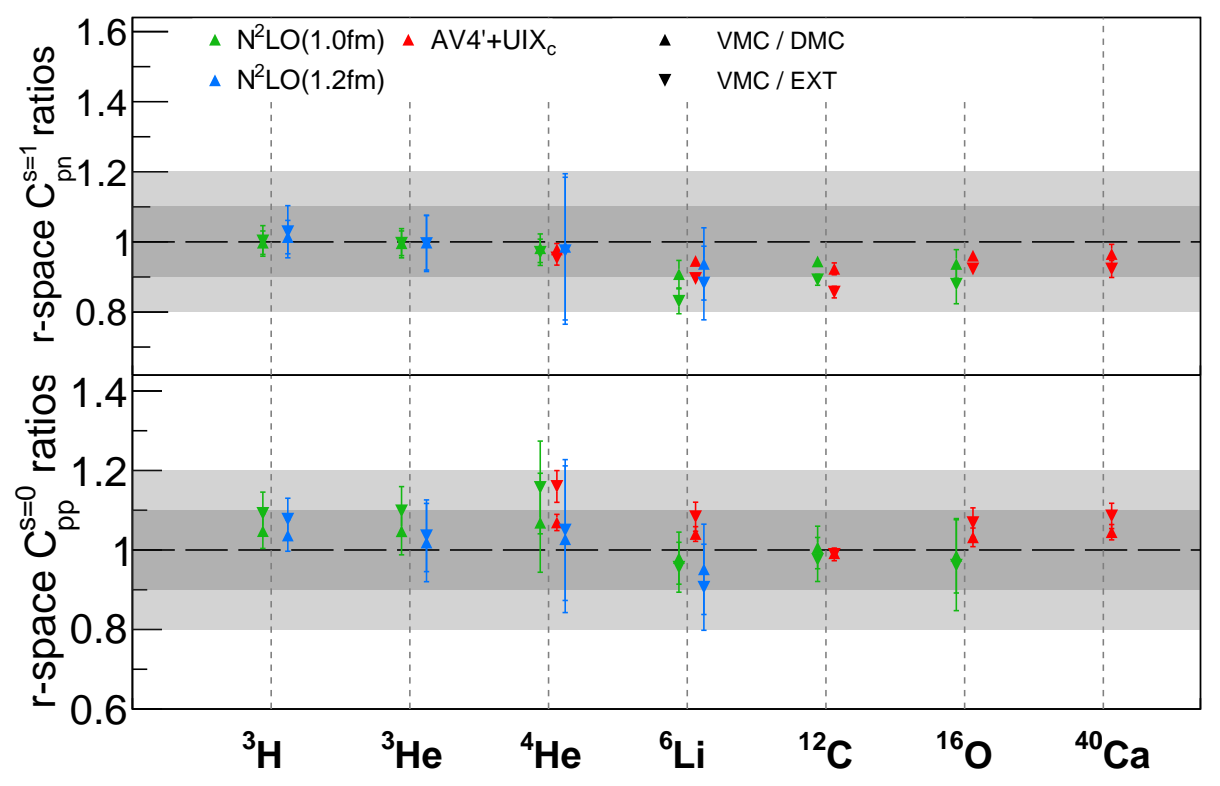

Figure 4-16: Ratios of contacts extracted from VMC 2-body coordinate densities to contacts extracted from DMC and EXT 2-body coordinate densities for the available nuclei and $N N$ interactions (see section 1.2 for details). The dark- and light-gray bands correspond to the $10 \%$ and $20 \%$ levels of agreement respectively.

\subsection{Applications of the GCF}

Besides the connections to atomic physics described at the beginning of this chapter (see Hen et al. [146]), the GCF ideas have found several additional applications. It has been used in studies of photo-absorption cross sections [147], symmetry energy of nucleonic matter and neutron stars [155], asymptotic behavior of the electronscattering Coulomb sum rule [156], nuclear charge densities [157], and the EMC effect [152.

Furthermore, the GCF has been used to calculate spectral functions in SRCdominated kinematics [158]. As explained in chapter 2, spectral functions are exactly calculable only for very light nuclei. For medium to heavy nuclei, spectral functions are determined based on effective theories, which typically lack the effects of ShortRange Correlations. Consequently, these GCF-based spectral functions allow one to calculate cross sections for the knock-out of high-momentum SRC nucleons and for the first time make rigorous quantitative comparisons to high-energy electron scattering data. Of specific interest is the GCF prediction that the repulsive core of the $N N$ 
interaction should become important for SRC pairs with relative momenta above $\approx$ $600 \mathrm{MeV} / c$. A recent analysis of CLAS data by my collaborators confirmed this prediction [63]. By examining the ratio of $\mathrm{A}\left(e, e^{\prime} p p\right)$ to $\mathrm{A}\left(e, e^{\prime} p\right)$ data as a function of $p_{\text {miss }}$, we found:

- A transition from $n p$-dominant region with few $A\left(e, e^{\prime} p p\right)$ events $\left(300<p_{\text {miss }}<\right.$ $600 \mathrm{MeV} / c)$ to a plateau $\left(p_{\text {miss }}>600 \mathrm{MeV} / c\right)$, indicating an isospin-independent interaction.

- The $A\left(e, e^{\prime} p p\right) / A\left(e, e^{\prime} p\right)$ ratio agrees with the GCF predictions for all $N N$ interactions used, except for the tensor-less AV4' interaction, as expected.

Follow-up $A\left(e, e^{\prime} p n\right)$ studies are underway [64]. The GCF-based spectral functions were also recently used to calculate $J / \psi$ photo-production cross sections [159].

Finally, the GCF allows us to extract nuclear correlation functions [140]. The procedure for doing so is described in the remainder of this chapter.

\subsection{Nuclear correlation functions}

The many-body probability density for a non-interacting system corresponds to the product of the single-particle probability densities of its components. In the presence of particle-particle correlations, there can be substantial deviations from this simple picture, which can be encapsulated in correlation functions. Since the atomic nucleus is a dense quantum-mechanical strongly-interacting many-body system, it cannot be described as a collection of non-interacting particles, particularly at small distances where SRCs dominate and, consequently, correlation functions are widely used in nuclear physics. The importance of correlation functions in the description of nuclear systems was introduced by R. Jastrow 34 and presented in section 1.3 of this thesis. One of the first nuclear correlation function parametrizations was determined by Miller and Spencer [160] and used for several decades in a variety of nuclear structure calculations. Some examples of studies that use nuclear correlation functions include calculations of: neutrinoless double-beta decay [21, 22, 23, 24, 161, 162], nuclear 
parity violation [163, 164], nuclear transparency in quasi-elastic scattering [165, 166, 167, 168, 169, 170], and shadowing in deep inelastic scattering [171. In spite of the spin and isospin dependence of the $N N$ interaction, which becomes very important mainly at short distances, and the widespread use of nuclear correlation functions, the study of the spin and isospin decomposition of correlation functions has received less attention. The determination of nuclear correlation functions is most important for medium and heavy nuclei. In this section, I will describe how we used the GCF to extract the spin and isospin decomposition of nuclear correlation functions for ${ }^{16} \mathrm{O}$ and ${ }^{40} \mathrm{Ca}$ (the two heaviest nuclei studied so far using Cluster VMC (CVMC)).

\subsubsection{Correlation function definition}

As mentioned above, the correlation function describes the deviation of the density of a many-body system from the simple picture in which all particles are uncorrelated. As such, the standard procedure for defining the nuclear correlation function $(\mathbb{F})$ for a given nucleus $(A)$ as a function of the separation between two nucleons $(r)$ is:

$$
\mathbb{F}_{N N}^{\alpha}(A, r) \equiv \frac{\rho_{N N}^{\alpha}(A, r)}{\rho_{N N}^{\alpha, \text { uncorr. }}(A, r)}
$$

where the numerator corresponds to a fully-correlated 2-body coordinate density, and the denominator corresponds to a 2-body coordinate density calculated in the absence of dynamical correlations.

\subsubsection{Fully-correlated 2-body coordinate density}

At short distances $(r \lesssim 1 \mathrm{fm})$ the spin and isospin decomposed fully-correlated 2body density can be accurately described using the GCF (see equation 4.10). At

long distances $(r \gtrsim 2 \mathrm{fm})$, nucleons should behave independently, and thus the fullycorrelated 2-body density can be approximated as the product of two (uncorrelated) single-nucleon densities integrated over the center-of-mass position $(\vec{R})$ of the pair:

$$
\rho_{N N}^{(0)}(A, \vec{r}) \equiv S_{N N} \int d \vec{R} \rho_{N}(A, \vec{R}+\vec{r} / 2) \rho_{N}(A, \vec{R}-\vec{r} / 2)
$$


Here, the single-nucleon densities are normalized to the proton or neutron number, and $S_{N N}$ represents a symmetry factor:

$$
S_{N N}= \begin{cases}1, & \text { for } p n \text { pairs } \\ Z(Z-1) / 2 Z^{2}, & \text { for } p p \text { pairs }(Z=\text { number of protons }) \\ N(N-1) / 2 N^{2}, & \text { for } n n \text { pairs }(N=\text { number of neutrons }) .\end{cases}
$$

At intermediate distances $(1 \lesssim r \lesssim 2 \mathrm{fm})$ both behaviors are present. Thus, in our model, we describe the full 2-body density by a combination of the short- and longdistance behaviors, with the relative contribution determined by a blending function $\left(g_{N N}(A, r)\right)$, and a constant $(\kappa)$ such that:

$$
\rho_{N N}^{\alpha}(A, r)=g_{N N}(A, r) \rho_{N N}^{\mathrm{GCF}}(A, r)+\kappa\left(1-g_{N N}(A, r)\right) \rho_{N N}^{(0)}(A, r) .
$$

When solving for the blending function one gets:

$$
g_{N N}(A, r)=\frac{\rho_{N N}^{\alpha}(A, r)-\kappa \rho_{N N}^{(0)}(A, r)}{\rho_{N N}^{\mathrm{GCF}}(A, r)-\kappa \rho_{N N}^{(0)}(A, r)} .
$$

While the most straightforward choice of $\kappa$ in equation 4.34 corresponds to 1 , this parameter is introduced to make sure the denominator in equation 4.35 never equals 0 and therefore the blending function is never singular.

In order to satisfy the condition that at short distances $\rho_{N N}^{\alpha}(A, r)$ be fully described by the GCF 2-body density $\left(\rho_{N N}^{\mathrm{GCF}}(A, r)\right)$, the blending function at short distances should satisfy $g_{N N}(A, r) \rightarrow 1$. Similarly, in order to satisfy the condition that at long distances $\rho_{N N}^{\alpha}(A, r)$ be fully described by the uncorrelated 2-body density $\left(\rho_{N N}^{(0)}(A, r)\right)$, since $\rho_{N N}^{\mathrm{GCF}}(A, r)$ falls as $1 / r^{2}$ for $r>2 \mathrm{fm}$, the blending function at long distances should satisfy $g_{N N}(A, r) \rightarrow(\kappa-1) / \kappa$. We proposed the following parametrization for the blending function: 


$$
g_{N N}(A, r)=\left\{\begin{array}{lc}
1, & r \leq 0.9 \mathrm{fm} \\
\frac{1}{\kappa}\left(\kappa-1+e^{(0.9 \mathrm{fm}-r) / a}\right), & r>0.9 \mathrm{fm},
\end{array}\right.
$$

where the short- and long-distance behaviors are blended with a characteristic lengthscale $a$, which should depend on the isospin of the pairs and on the specific nucleus being studied. This parameter (and thus the entire blending function) can be determined by fitting equation 4.34 to fully correlated 2-body coordinate densities calculated from CVMC [16]. We carried out an independent study for $p p, n n$, and $p n$ pairs in ${ }^{16} \mathrm{O}$ and ${ }^{40} \mathrm{Ca}$ and learned that the blending function is both isospin and nucleus independent, and thus $g_{N N}(A, r) \rightarrow g(r)$. We find that $a$ depends smoothly on $\kappa$, and for $\kappa=2$ it is determined to be $a=1.518 \pm 0.001 \mathrm{fm}$.

We verified the validity of the density extracted using this model with that from CVMC, as shown in Fig. 4-17.

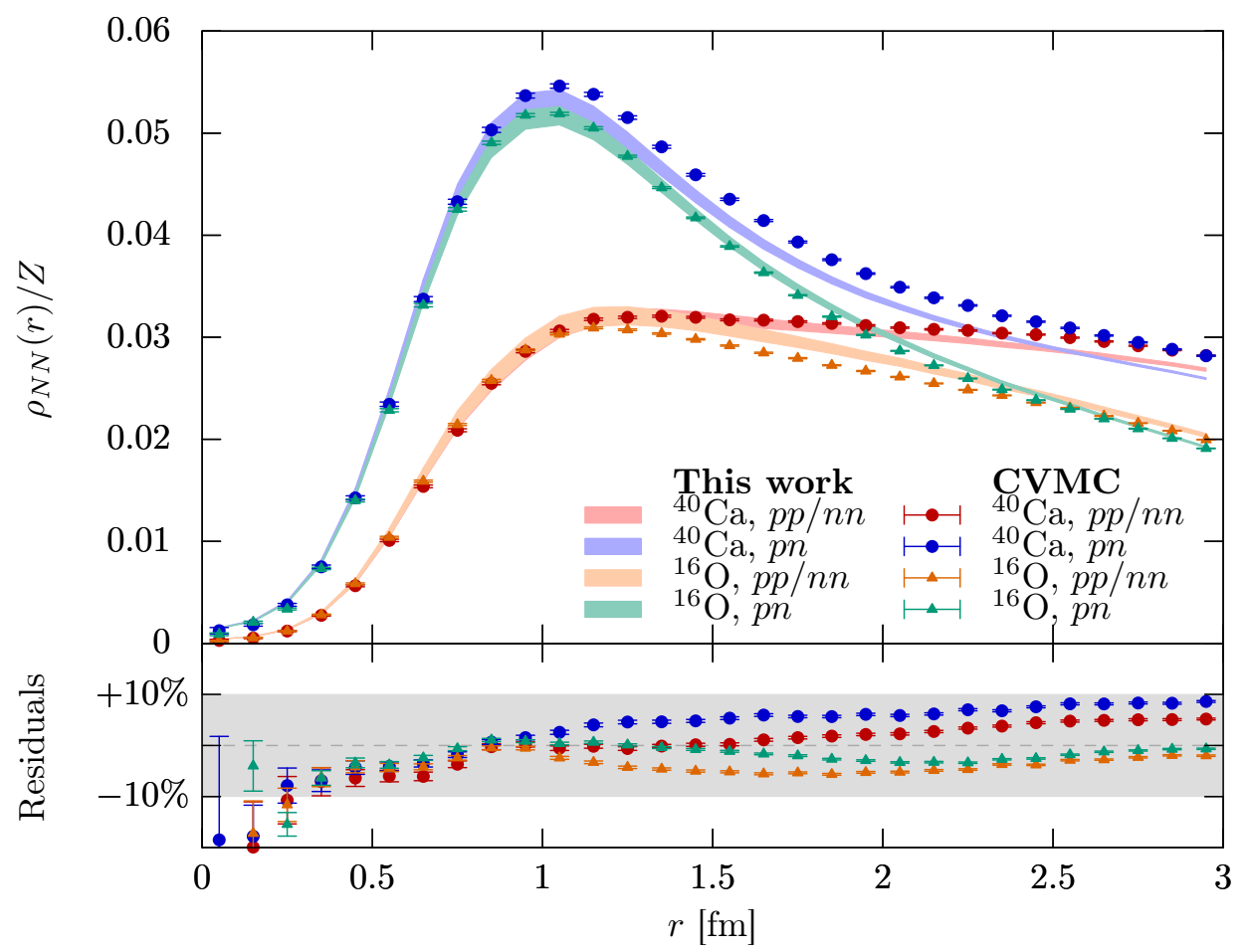

Figure 4-17: Comparison between the 2-body coordinate density from the model from equation 4.34 (shown as bands for $\kappa=2$ and $a=1.518 \pm 0.001 \mathrm{fm}$ ) and that from CVMC (shown as markers) for ${ }^{16} \mathrm{O}$ and ${ }^{40} \mathrm{Ca}$. 
The dominant source of uncertainty comes from the contact coefficients. Our model reproduces the fully correlated 2-body coordinate density in these two nuclei for all types of pairs (since ${ }^{16} \mathrm{O}$ and ${ }^{40} \mathrm{Ca}$ are symmetric, the $p p$ and $n n$ behavior is the same) to about $10 \%$. This shows that the spin-isospin dependence of the 2-body coordinate density is dominant at short distances (and thus originates from the GCF densities) while the long-range behavior is universal for a given nucleus.

The single-nucleon density used to calculate the uncorrelated 2-body density from equation 4.32 is taken from CVMC. Nevertheless, these single-nucleon densities have been well-constrained experimentally, and several parametrizations for different nuclei can be found in the literature. See, for example, [172].

\subsubsection{Uncorrelated 2-body coordinate density and the Pauli exclusion principle}

To build the correlation function, we must now proceed to determine the denominator in equation 4.31. While this denominator corresponds to an uncorrelated 2-body coordinate density, this quantity must be treated with more sophistication than the one presented in equation 4.32 the correlative effects of the Pauli exclusion principle must be included.

Typically, uncorrelated 2-body coordinate densities are determined from antisymmetrized wave functions in the form of a Slater determinant:

$$
\begin{aligned}
\rho_{i j}^{\text {uncorr. }}(\vec{r})=\frac{1}{2} \sum_{\alpha, \beta, \in \text { occ }} \int \mathrm{d}^{3} r_{i} \mathrm{~d}^{3} r_{j} \delta\left(\vec{r}-\left(\vec{r}_{i}\right.\right. & \left.\left.-\vec{r}_{j}\right)\right) \phi_{\alpha}^{\dagger}\left(x_{i}\right) \phi_{\beta}^{\dagger}\left(x_{j}\right) \\
& \times\left[\phi_{\alpha}\left(x_{i}\right) \phi_{\beta}\left(x_{j}\right)-\phi_{\beta}\left(x_{i}\right) \phi_{\alpha}\left(x_{j}\right)\right],
\end{aligned}
$$

where $\alpha$ and $\beta$ are isospin labels and $x_{i}$ represents the pair quantum numbers: $x \equiv$ $\left(\vec{r}, m_{s}= \pm 1 / 2, m_{t}= \pm 1 / 2\right)$. In the case of $p n$ pairs, this expression reduces to that from equation 4.32. However, for the case of $p p$ pairs, two spin-up protons cannot 
occupy the same orbital (Pauli principle). Thus one gets:

$$
\begin{aligned}
\rho_{p p}^{\text {uncorr. }}(\vec{r})= & \frac{1}{2} \int \mathrm{d}^{3} r_{1} \mathrm{~d}^{3} r_{2} \delta\left(\vec{r}-\left(\vec{r}_{1}-\vec{r}_{2}\right)\right) \\
& \times\left[\rho\left(\vec{r}_{1}\right) \rho\left(\vec{r}_{2}\right)-\frac{1}{2} \rho\left(\vec{r}_{1}, \vec{r}_{2}\right) \rho\left(\vec{r}_{2}, \vec{r}_{1}\right)\right] \\
\equiv & \frac{Z}{Z-1} \rho_{p p}^{(0)}(\vec{r})-\rho_{p p}^{\text {exch. }}(\vec{r}),
\end{aligned}
$$

where $\rho(\vec{r})$ is the single-nucleon proton density normalized to $Z$. The quantity $\rho\left(\vec{r}_{1}, \vec{r}_{2}\right)$ is the density-matrix defined such that its diagonal elements yield the proton or neutron single-nucleon density. The second term of 4.39 represents the influence of the Pauli exclusion principle. The same happens for $n n$ pairs. The expression in this case is obtained by substituting $N$ for $Z$ and the single-nucleon neutron density for the proton one.

We use a result based on nuclear matter (but using the local-density approximation to the first term of the density-matrix expansion of [173]) for $\rho_{p p}^{\text {exch. }}(\vec{r})$ (the alternative would be to use a nucleus-specific Slater determinant):

$$
\rho_{p p}^{\text {exch. }}(\vec{r})=\frac{Z}{2(Z-1)} \rho_{p p}^{(0)}(r) \times\left(\frac{3 j_{1}\left(\bar{k}_{F} r\right)}{\bar{k}_{F} r}\right)^{2}
$$

where $\bar{k}_{F}=200 \mathrm{MeV} / \mathrm{c}$ is a Fermi momentum averaged over the nuclear volume and $j_{1}$ is a spherical Bessel function. We verified the accuracy of equation 4.40 numerically, by comparing with the Slater determinant provided by the single-particle wave functions of [174].

The effect of including or neglecting the term $\rho_{p p}^{\text {exch. }}(\vec{r})$ in equation 4.39 when calculating the $p p$ correlation function can be seen in Fig. 4-18. The effect of the Pauli principle is to strongly reduce the uncorrelated density at small distances, thus enhancing the $p p$ and $n n$ correlation function. It is crucial to keep track of the Pauli principle effects when comparing correlation functions from different authors. 


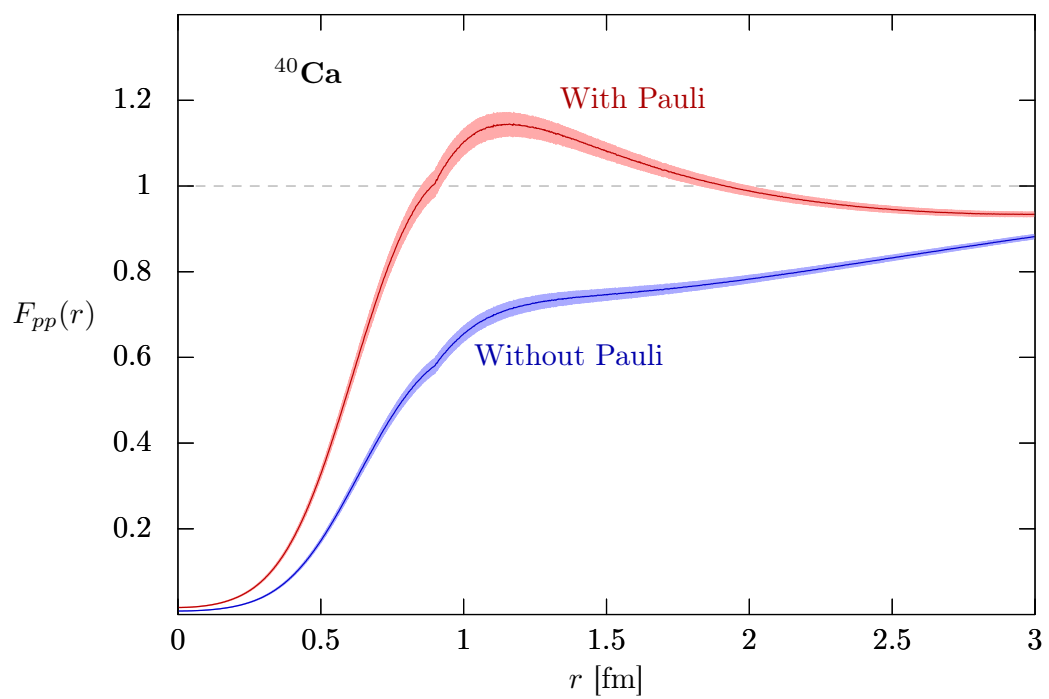

Figure 4-18: Effect of including or neglecting the term $\rho_{p p}^{\text {exch. }}(\vec{r})$ in equation 4.39 when calculating the $p p$ correlation function, shown for ${ }^{40} \mathrm{Ca}$.

\subsubsection{Correlation function results and discussion}

We now have all the components necessary to extract the nuclear correlation function from equation 4.31. The results from our model for ${ }^{16} \mathrm{O}$ and ${ }^{40} \mathrm{Ca}$ for all types of pairs (since these two nuclei are symmetric, the $p p$ and $n n$ behavior is the same) are shown as colored bands in Fig. 4-19. The equivalent results from CVMC are shown as points of the same colors. The correlation functions are qualitatively similar for both nuclei and different types of pairs, with some isospin dependence mainly at short distances $(r<1.5 \mathrm{fm})$. These differences are caused by the fact that, at short distances, $p p$ (and $n n$ ) pairs are predominantly spin-0 while $p n$ pairs are predominantly spin-1.

For comparison and reference, Fig. 4-19 also shows several other calculations of nuclear correlation functions. The correlation functions from CVMC and from our model are close to that of Simkovic et al. [24] and to the ${ }^{16} \mathrm{O}$ calculations of Alvioli et al., but are higher than the correlation functions predicted by Benhar et al. and by Miller and Spencer. The calculations by Alvioli et al. for ${ }^{40} \mathrm{Ca}$ predict a significantly higher correlation function for both $p p / n n$ and $p n$. The calculation using the Unitary Correlation Operator Method (UCOM) [176] in the $T=0, S=1$ channel, shown as a black dash-dotted line, is slightly lower than our predictions for pn pairs. 


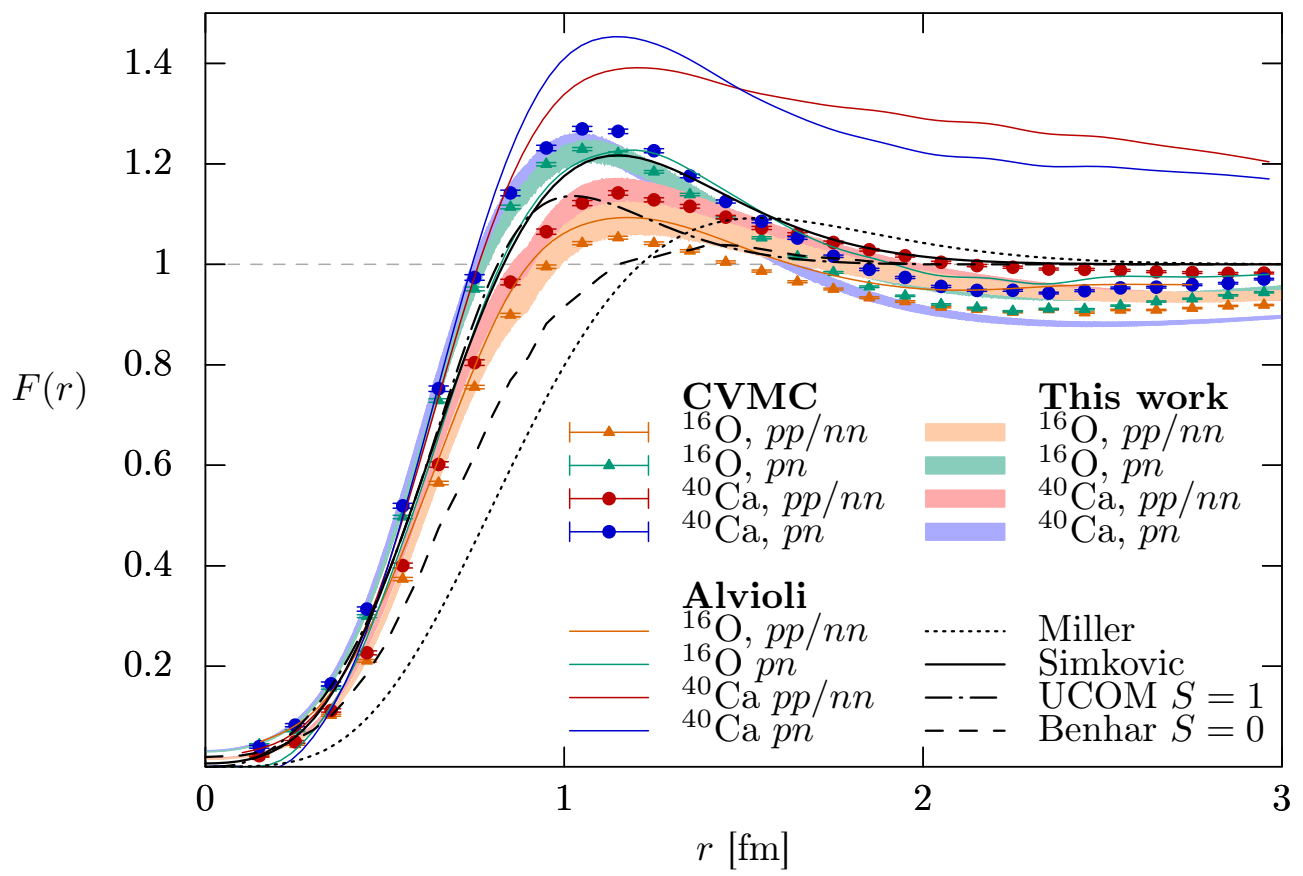

Figure 4-19: Results from the isospin-decomposed ${ }^{16} \mathrm{O}$ and ${ }^{40} \mathrm{Ca}$ correlation functions and comparison to several other calculations. The black dotted line (........) corresponds to the original model suggested by Miller and Spencer [160]. The colored thin lines correspond to calculations by Alvioli et al. [175]. The black dashed line (---- ) corresponds to calculations by Benhar et al. [25]. The black solid line corresponds to calculations by Simkovic et al. 24]. The black dash-dotted line (-.-) corresponds to a calculation using the Unitary Correlation Operator Method (UCOM) [176] in the $T=0, S=1$ channel. See text for details. 
A study of Jastrow correlation functions [177] shows that isospin symmetry is broken at the level of two-body cluster truncation. This is one of the problems with the Miller-Spencer parameterization. However, the Simkovic et al. model avoids this problem because of the bump (at about $r=1 \mathrm{fm}$ ) in their correlation function. Our agreement with the Simkovic et al. model shows that our work also avoids this problem.

As shown in section 4.4.3, nuclear contacts can be extracted from experimental data. This implies that one can potentially extract contacts, and subsequently 2body coordinate densities and nuclear correlation functions for heavier nuclei for which QMC calculations are not available.

\section{Correlation function parametrization}

For ease of implementation, we provide the following parametrization for the correlation function from our model:

$$
\mathbb{F}(r)=1-e^{-\alpha r^{2}} \times\left(\gamma+r \sum_{i=1}^{3} \beta_{i} r^{i}\right)
$$

with parameter values given in Table 4.3. This function reproduces the correlation functions of both ${ }^{16} \mathrm{O}$ and ${ }^{40} \mathrm{Ca}$.

Table 4.3: Parameters describing $\mathbb{F}(r)$, using the functional form of equation 4.41.

\begin{tabular}{c|ccc} 
Parameter & Units & Value $(p p / n n)$ & Value $(p n)$ \\
\hline \hline$\alpha$ & $\mathrm{fm}^{-2}$ & 3.17 & 1.08 \\
$\gamma$ & - & 0.995 & 0.985 \\
$\beta_{1}$ & $\mathrm{fm}^{-2}$ & 1.81 & -0.432 \\
$\beta_{2}$ & $\mathrm{fm}^{-3}$ & 5.90 & -3.30 \\
$\beta_{3}$ & $\mathrm{fm}^{-4}$ & -9.87 & 2.01
\end{tabular}




\section{Chapter 5}

\section{Summary and Conclusions}

In chapter 3 , I presented the first simultaneous measurement of the ${ }^{3} \mathrm{He}$ and ${ }^{3} \mathrm{H}\left(e, e^{\prime} p\right)$ reactions in kinematics where non-QE reaction mechanisms should be minimal, and hence the cross sections should be sensitive to the proton momentum distributions. These kinematics correspond to large $Q^{2}, x_{B}>1$, and $\theta_{r q}<40^{\circ}$. Further sensitivity to the momentum distributions is expected in the ${ }^{3} \mathrm{He} /{ }^{3} \mathrm{H}$ cross-section ratio, since any residual FSI effect should cancel in equal-mass nuclei (as confirmed by a generalized Eikonal approximation calculation of the struck-nucleon single rescattering). We found that, while the ${ }^{3} \mathrm{He} /{ }^{3} \mathrm{H}$ cross-section ratio well describes the ratio of momentum distributions up to $p_{\text {miss }} \approx 250 \mathrm{MeV} / c$, they disagree by $20-50 \%$ at higher $p_{\text {miss }}$. None of the currently available calculations can describe this result. Consequently, to study whether the high- $p_{\text {miss }}$ discrepancy came from additional non-QE reaction mechanisms, or from deficiencies in the $A=3$ wave functions, and also to test several state-of-the-art $A=3$ cross-section-calculation models, we then extracted the ${ }^{3} \mathrm{He}$ and ${ }^{3} \mathrm{H}\left(e, e^{\prime} p\right)$ absolute cross sections. We found the measured and PWIA-calculated ${ }^{3} \mathrm{H}$ cross sections agreed to within $10-20 \%$. On the other hand, the measured and PWIA-calculated ${ }^{3} \mathrm{He}$ cross sections only agreed for $150<p_{\text {miss }}<350 \mathrm{MeV} / c$. Including FSI of the struck nucleon in the theory calculation improved the agreement for both ${ }^{3} \mathrm{He}$ and ${ }^{3} \mathrm{H}$ at high- $p_{\text {miss }}$. However, even after including FSI, we observed that the ${ }^{3} \mathrm{He}$ and ${ }^{3} \mathrm{H}$ measured cross sections increase and decrease respectively relative to theory calculations at high- $p_{m i s s}$. This observation is consistent with the 
hypothesis that single-charge exchange (SCX) at high- $p_{m i s s}$ can explain the remaining differences between the measured cross sections and calculations. Nevertheless, additional calculations that quantify the SCX effect are needed. Finally, we found that the PWIA-calculations agreement with our measurement is significantly better than with previous ${ }^{3} \mathrm{He}\left(e, e^{\prime} p\right)$ measurements carried out at $Q^{2}=1.5\left(\mathrm{GeV} / c^{2}\right)^{2}$ and $x_{B}=1$, where non-QE reaction mechanisms dominated the cross section, and the interpretation of the extracted results was consequently limited [42, 44]. The isoscalar $\left({ }^{3} \mathrm{He}+{ }^{3} \mathrm{H}\right)$ cross section is well described by the available calculations, validating current models of the $A=3$ ground state up to missing momenta of 500 $\mathrm{MeV} / c$.

In chapter 4, I introduced the atomic Contact Formalism, and subsequently overviewed its generalization to nuclear systems. As a result of this study we learned that, even though nuclei don't strictly satisfy the scale-separation requirements of this formalism, its application to study SRCs in nuclei yields results that are consistent both with Quantum Monte Carlo (QMC) calculations at short distances and high momenta (carried out using four different $N N$-interaction models), and with experimental SRC data to about $10-20 \%$. The GCF describes the short-distance and high-momentum behavior of nuclei as a linear combination of 2-body densities scaled by constants. The 2-body densities are taken to be the zero-energy solution of the Schrödinger equation for a pair of nucleons with a set of quantum numbers and, for a given $N N$ potential, are universal. The scaling constants, referred to as "contacts", carry the nucleus dependence and describe the abundance of pairs of a given set of quantum numbers in a given nucleus. The obtained equivalence between contacts independently extracted in coordinate and momentum spaces indicates quantitative agreement between the short-distance and high-momentum scaling in nuclei. The experimentally-verified $n p$ dominance in SRCs is naturally obtained in the GCF. Analysis of the obtained contact values reveals the spin-isospin content and also the non-combinatorial spin-isospin symmetry of SRCs. Agreement between contacts extracted from QMC calculations and experimental data opens up the possibility to, given new measurements, extract contacts for heavy nuclei for which QMC calculations do not exist. 
The GCF was used to study QMC calculations carried out both in coordinate and momentum space using four different $N N$-interaction models to identify scale and scheme independent SRC properties. Even though the GCF 2-body asymptotic densities are strongly scale and scheme dependent, nuclear contact ratios of nucleus $A$ to $\mathrm{d}$ or ${ }^{4} \mathrm{He}$ extracted both at short distances and at high momenta are largely scale and scheme independent. This implies that SRC relative abundances (and thus their formation mechanisms) are a Mean-Field (long-range) property. Beyond contact ratios, absolute contacts are also largely scale and scheme independent, with the exception of $p n$ spin-1 contacts with the AV4' interaction, and spin-0 contacts with the NV2+3-Ia* interaction and in coordinate space with the $\mathrm{N}^{2} \mathrm{LO}$ interaction. The former is expected (due to the AV4' interaction being tensor-less), while the latter is less well understood and will be the focus of future GCF studies. Other than the spin-0 case in the $\mathrm{N}^{2} \mathrm{LO}$ interaction, all the remaining channels (including the spin-1 case in the $\mathrm{N}^{2} \mathrm{LO}$ interaction) exhibit equivalence between the short-distance and high-momentum SRC scaling. These results pose direct implications for future experimental studies of SRC abundances and distributions in nuclei.

\subsection{Outlook}

The experimental and phenomenological studies presented in this thesis constitute only a step in the much larger endeavor to learn about the short-distance / highmomentum structure of the $N N$ interaction through the study of SRCs and vice versa. The effect of SCX on the ${ }^{3} \mathrm{He}$ and ${ }^{3} \mathrm{H}\left(e, e^{\prime} p\right)$ cross sections at large $p_{\text {miss }}$ are still to be studied. Furthermore, the studied models should be used to calculate the cross sections using different models of the $N N$ interaction in order to assess the potential dependence. The result from the ${ }^{3} \mathrm{He}$ and ${ }^{3} \mathrm{H}\left(e, e^{\prime} p\right)$ cross-section measurement constitutes, to date, the new benchmark for future few-body nuclear-theory calculations. The GCF is currently one of the most commonly-used effective theories to describe SRCs in nuclei. In addition to the applications described in this thesis, the GCF is currently being implemented in a cross-section generator to study $\mathrm{QE}$ 
$\left(e, e^{\prime} p\right)$ scattering off SRC nucleons in the future Electron-Ion Collider. Lastly, QMC calculations of 2-body densities as a function of relative and center-of-mass coordinates $(r, R)$ were recently carried out. Thus, in the future, nuclear contacts will be extracted in more detail as a function of $R$, revealing information on how SRC pairs distribute themselves throughout the nuclear volume. 


\section{Appendix A}

\section{HRS optics study}

\section{A.1 High-order HRS optics checks}

Study of Hall-A HRS optics by analyzing the relationship between physical variables from elastic $\mathrm{H}\left(e, e^{\prime} p\right)$ scattering (invariant mass, missing energy, and missing momentum components) and spectrometer optics variables $\left(\delta, \phi_{t g}, \theta_{t g}, y_{t g}\right)$. The y-axis variables have physical meaning and therefore must be independent of the HRS optics. See section 3.3 .2 for more details. The following plots exhibit this independence to within approximately $1 \mathrm{MeV}$.
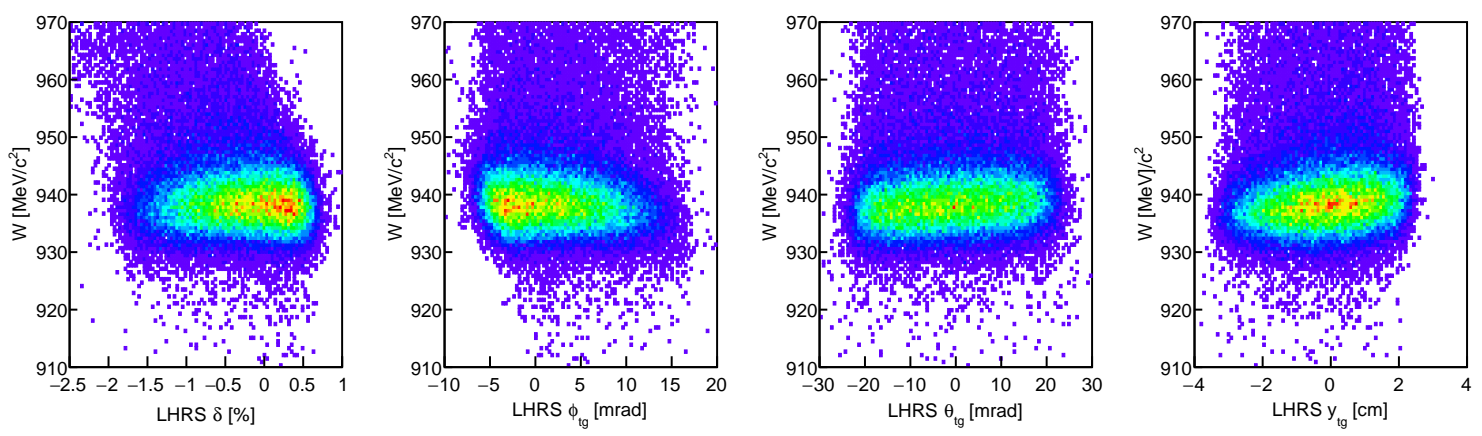

Figure A-1: Elastic $\mathrm{H}\left(e, e^{\prime} p\right)$ invariant mass as a function of LHRS optics variables. 

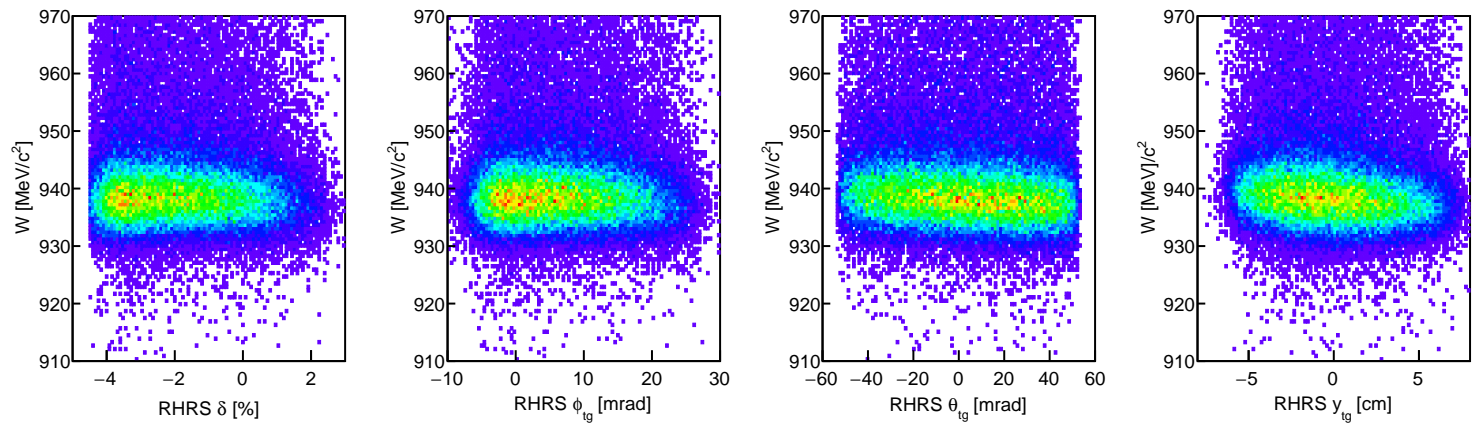

Figure A-2: Same as Fig. A-1 as a function of RHRS optics variables.
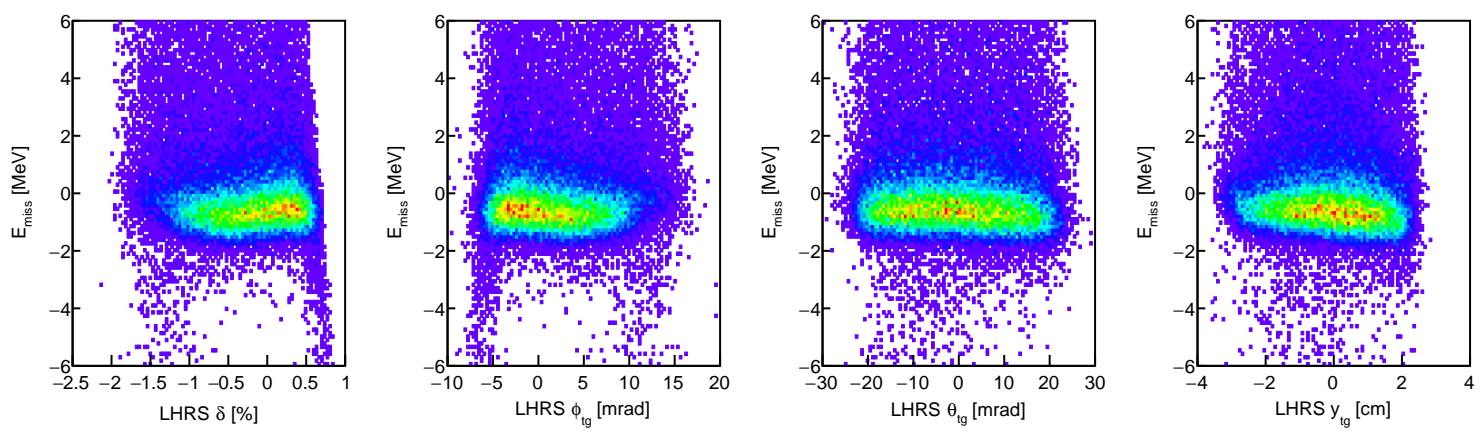

Figure A-3: Elastic $\mathrm{H}\left(e, e^{\prime} p\right)$ missing energy as a function of LHRS optics variables.
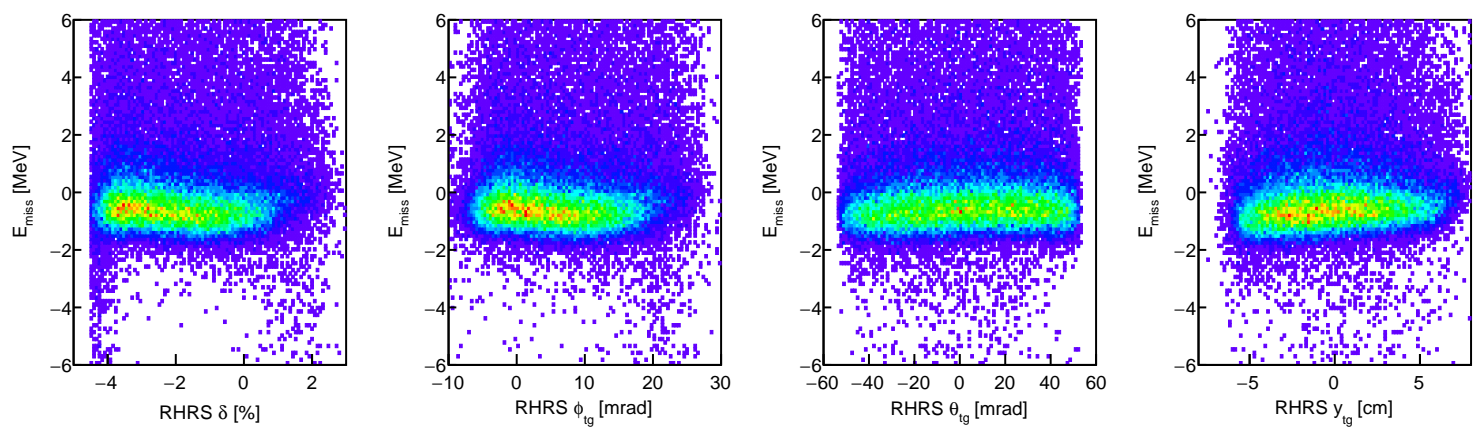

Figure A-4: Same as Fig. A-3 as a function of RHRS optics variables. 

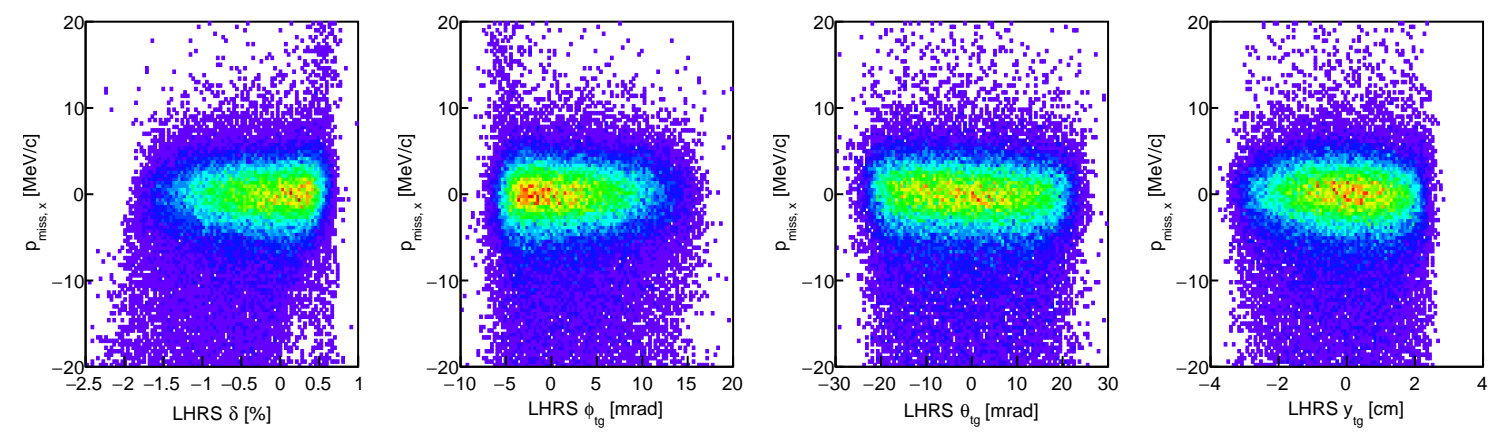

Figure A-5: Elastic $\mathrm{H}\left(e, e^{\prime} p\right) x$ component of missing momentum as a function of LHRS optics variables.
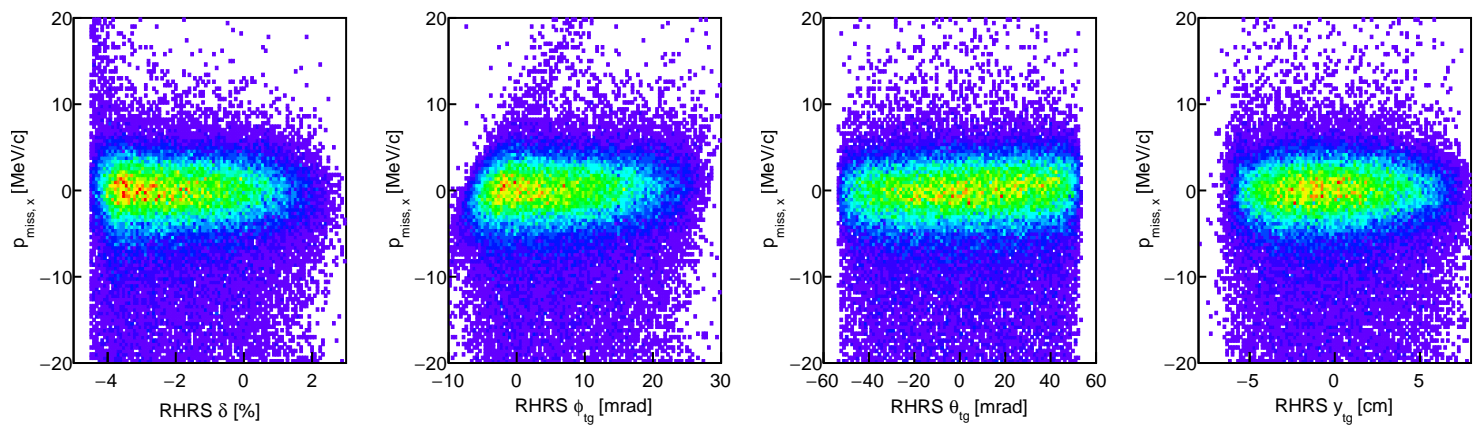

Figure A-6: Same as Fig. A-5 as a function of RHRS optics variables.
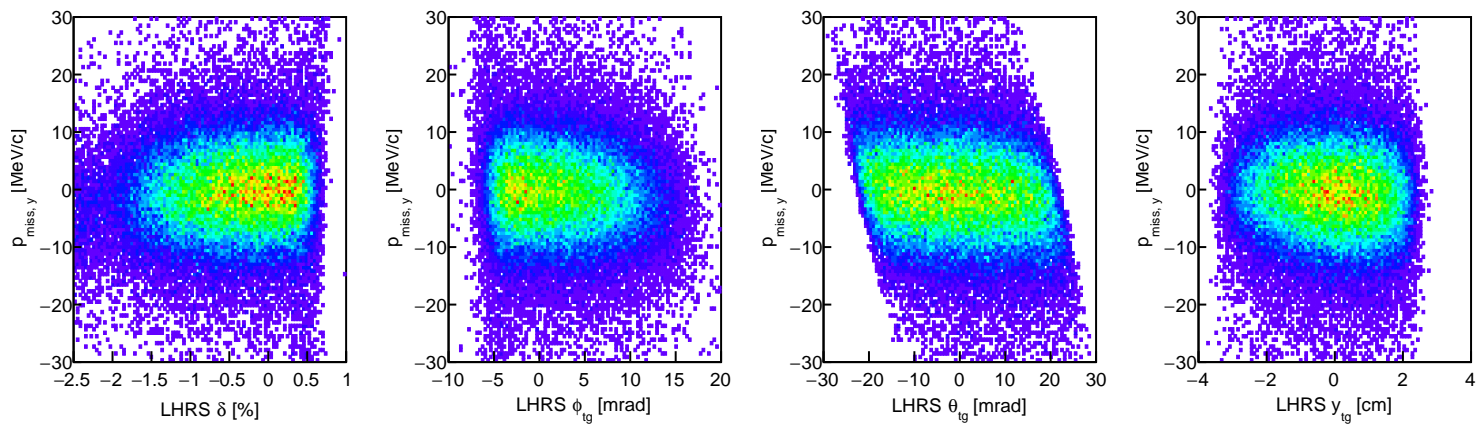

Figure A-7: Elastic $\mathrm{H}\left(e, e^{\prime} p\right)$ y component of missing momentum as a function of LHRS optics variables. 

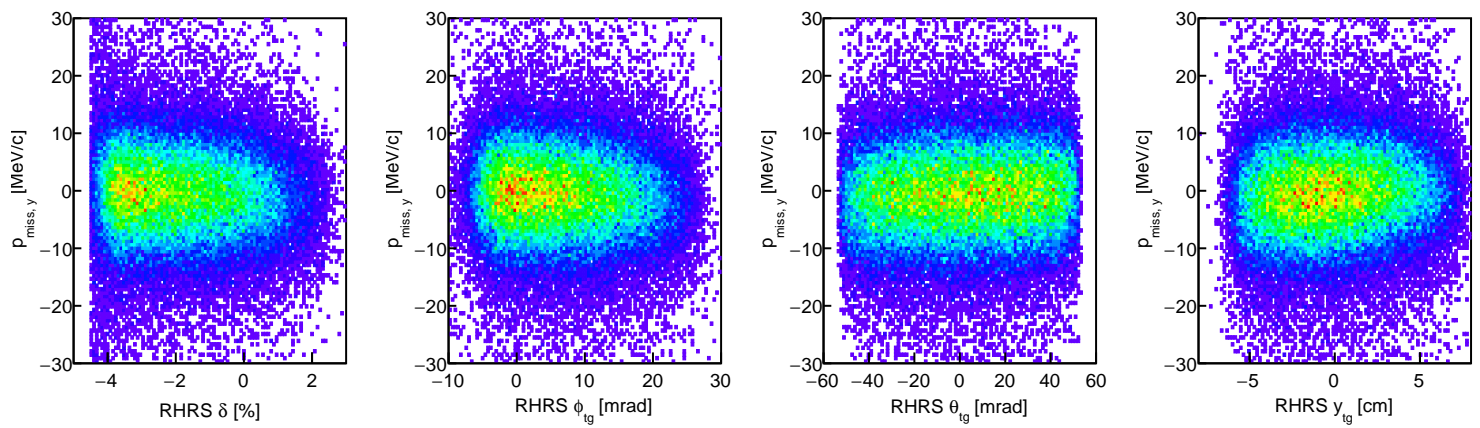

Figure A-8: Same as Fig. A-7 as a function of RHRS optics variables.
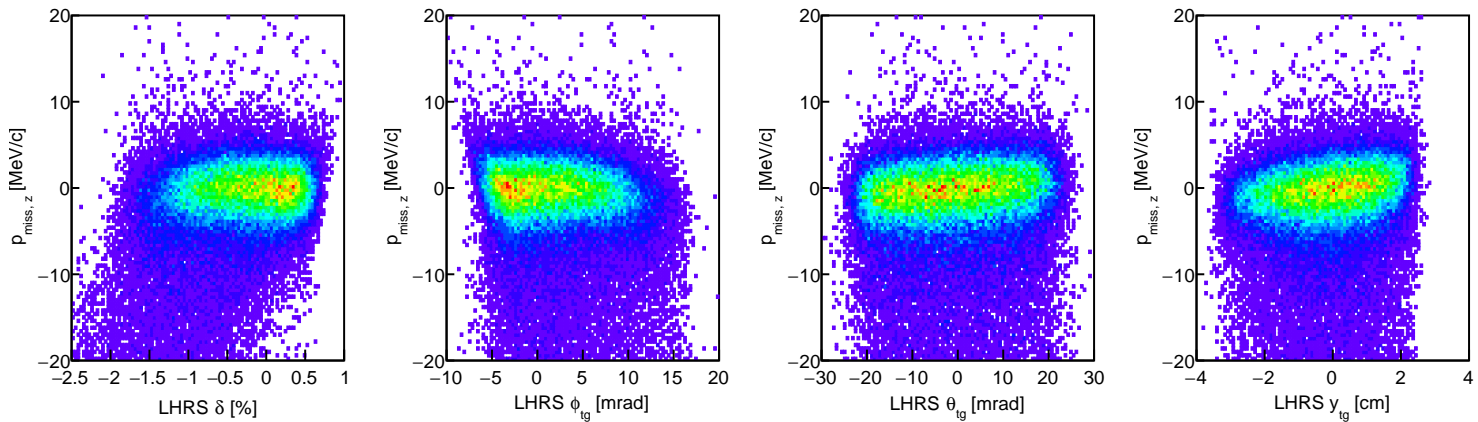

Figure A-9: Elastic $\mathrm{H}\left(e, e^{\prime} p\right) z$ component of missing momentum as a function of LHRS optics variables. 

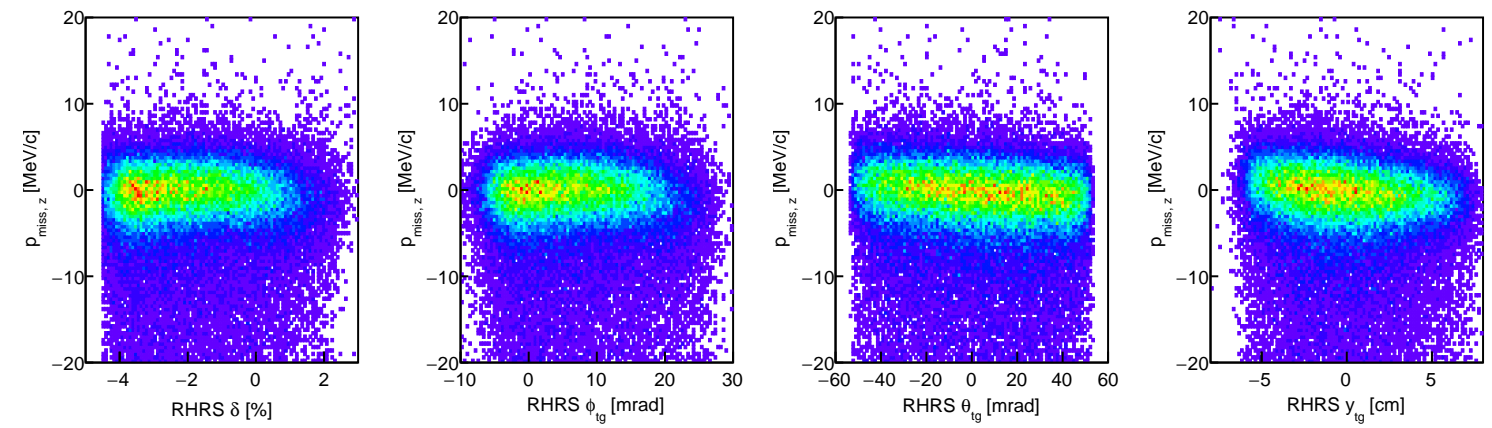

Figure A-10: Same as Fig. A-9 as a function of RHRS optics variables.

\section{A.2 $\quad 0^{t h}$-order HRS optics check}

Difference between variables measured by the High-Resolution Spectrometers (reco), and calculated from different pairs of variables (calc). The plots on the left show the distributions before any '0th' order correction. The plots on the right show the status after all the corrections described in section 3.3.2. All the distributions in a given plot have been normalized to have the same height at their mean value.
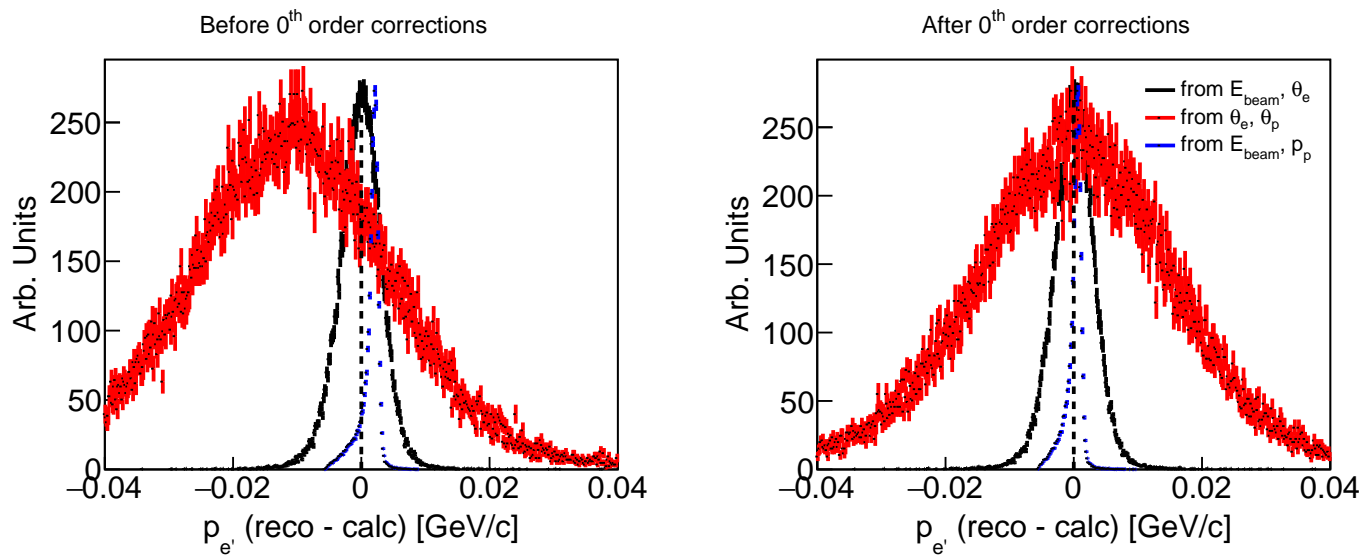

Figure A-11: Counts vs. scattered electron momentum. 

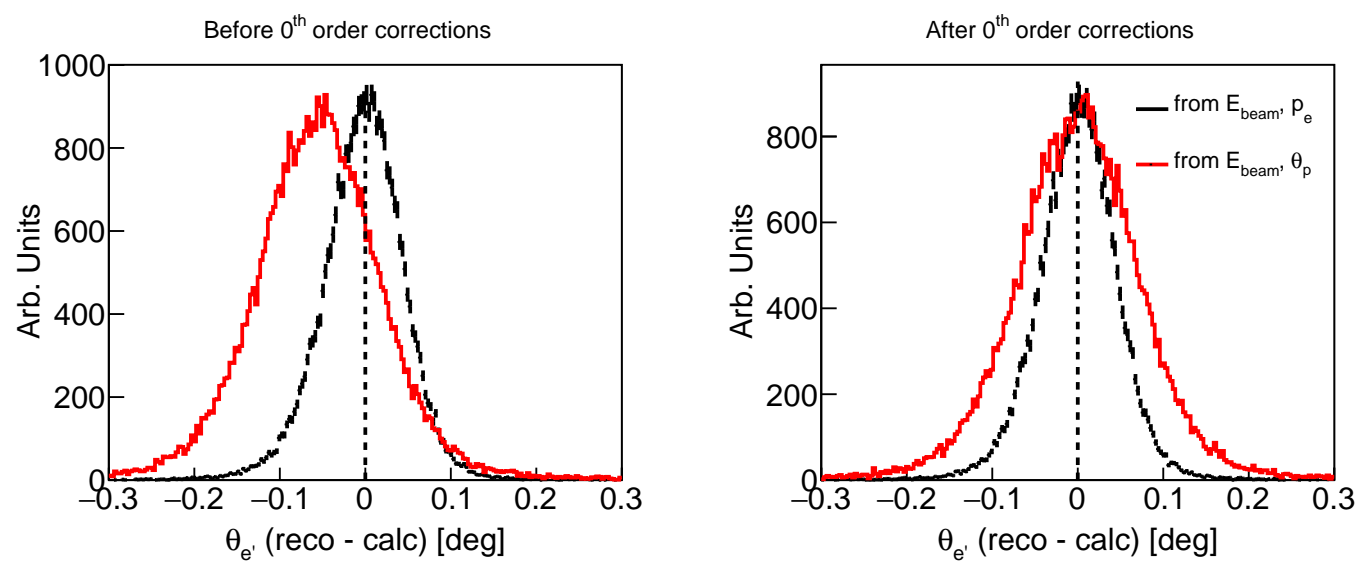

Figure A-12: Counts vs. electron scattering angle.
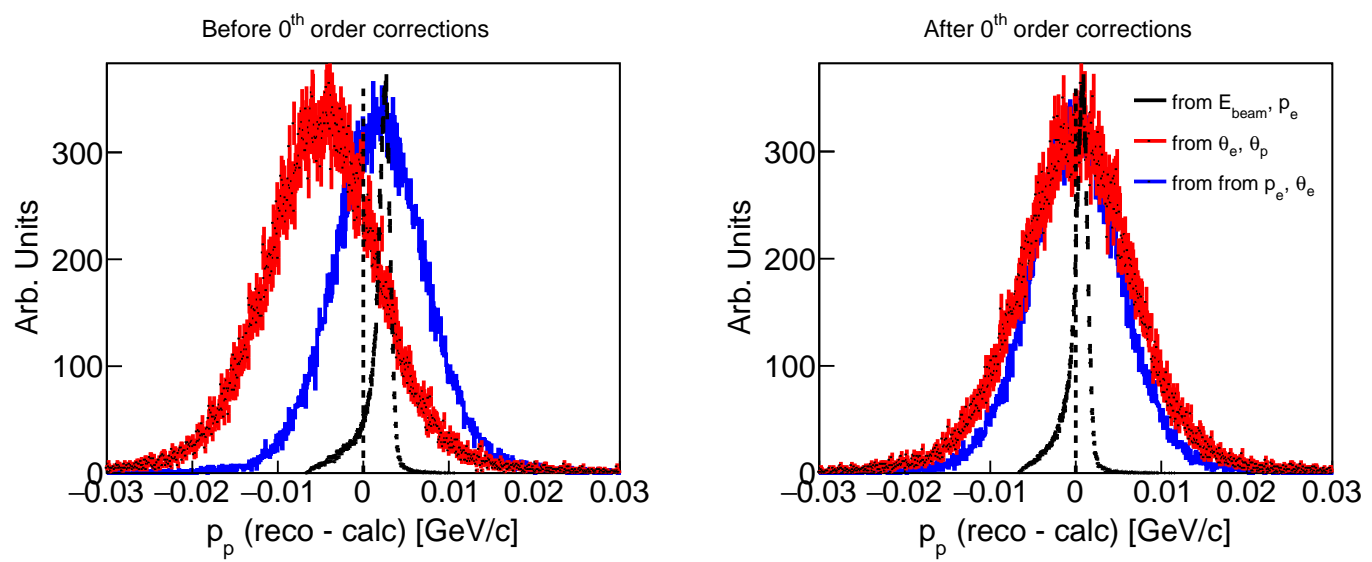

Figure A-13: Counts vs. knocked-out proton momentum.

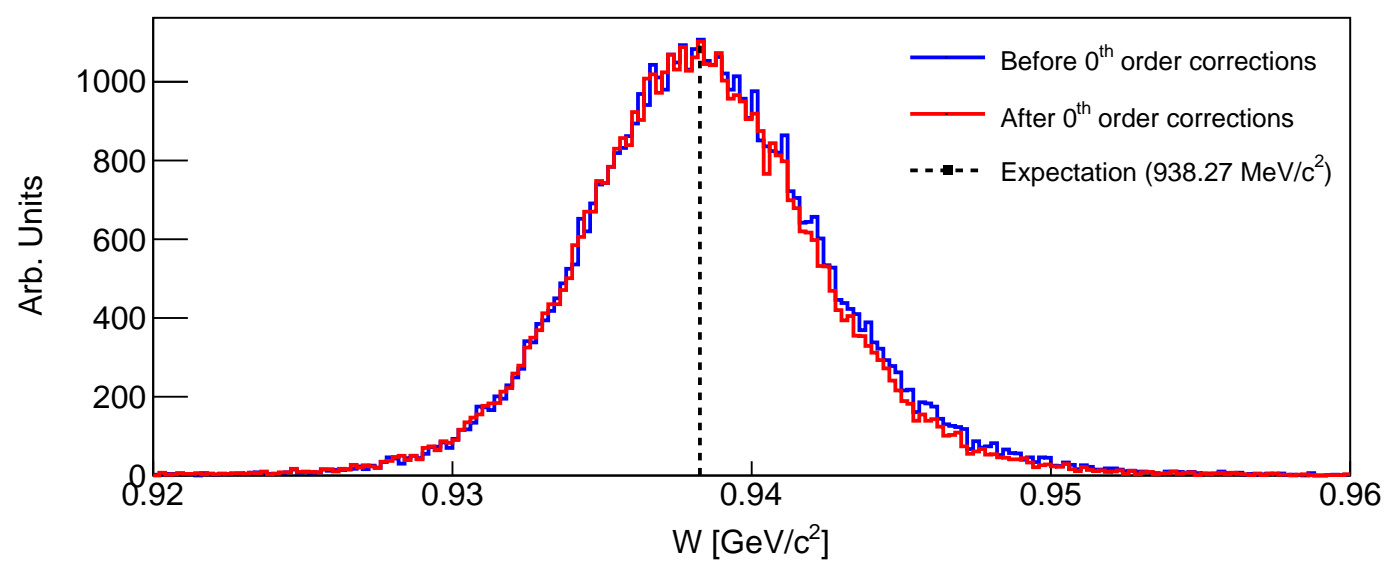

Figure A-14: Counts vs. invariant mass. 


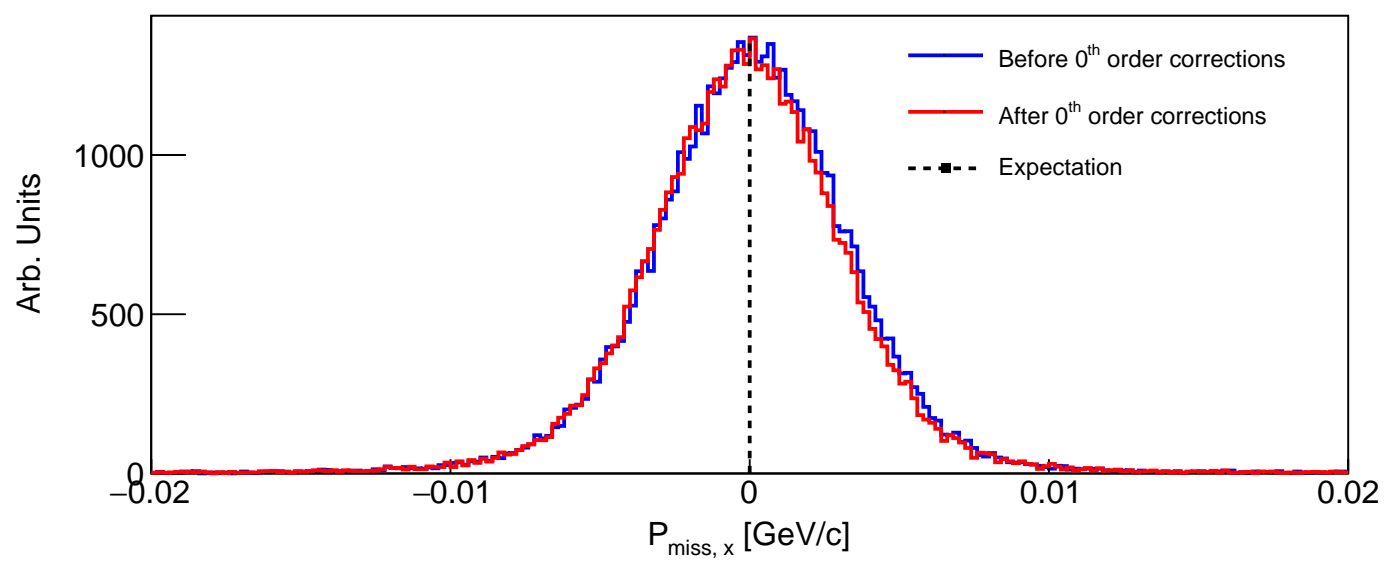

Figure A-15: Counts vs. missing momentum $x$ component.

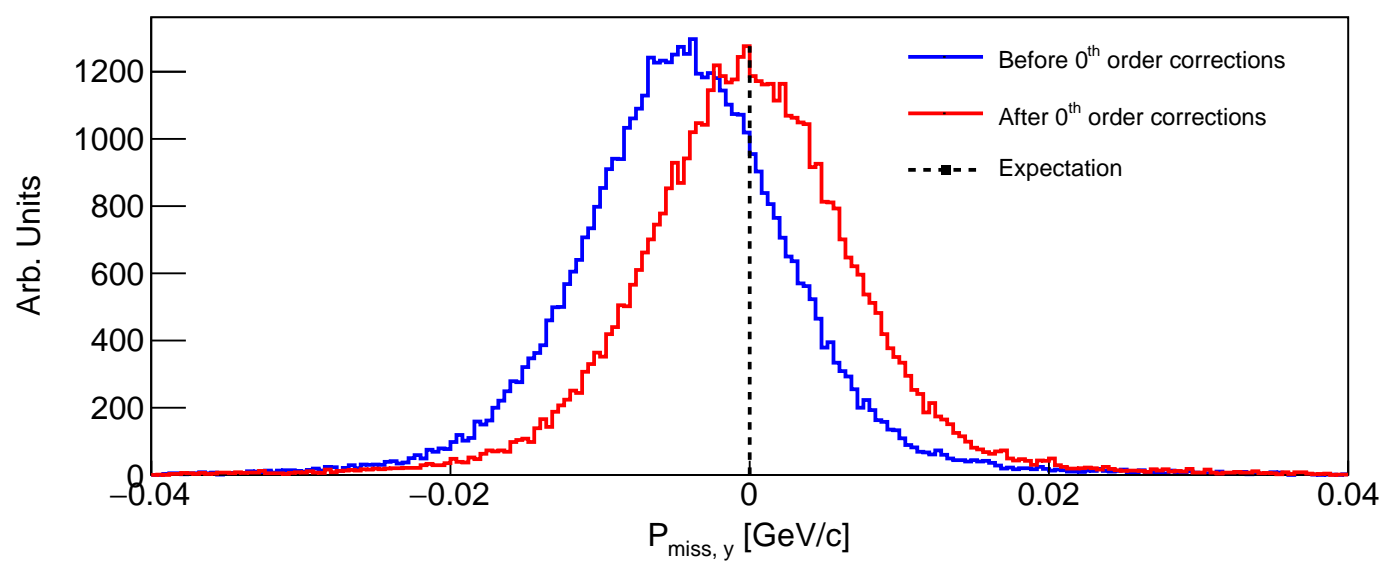

Figure A-16: Counts vs. missing momentum $y$ component.

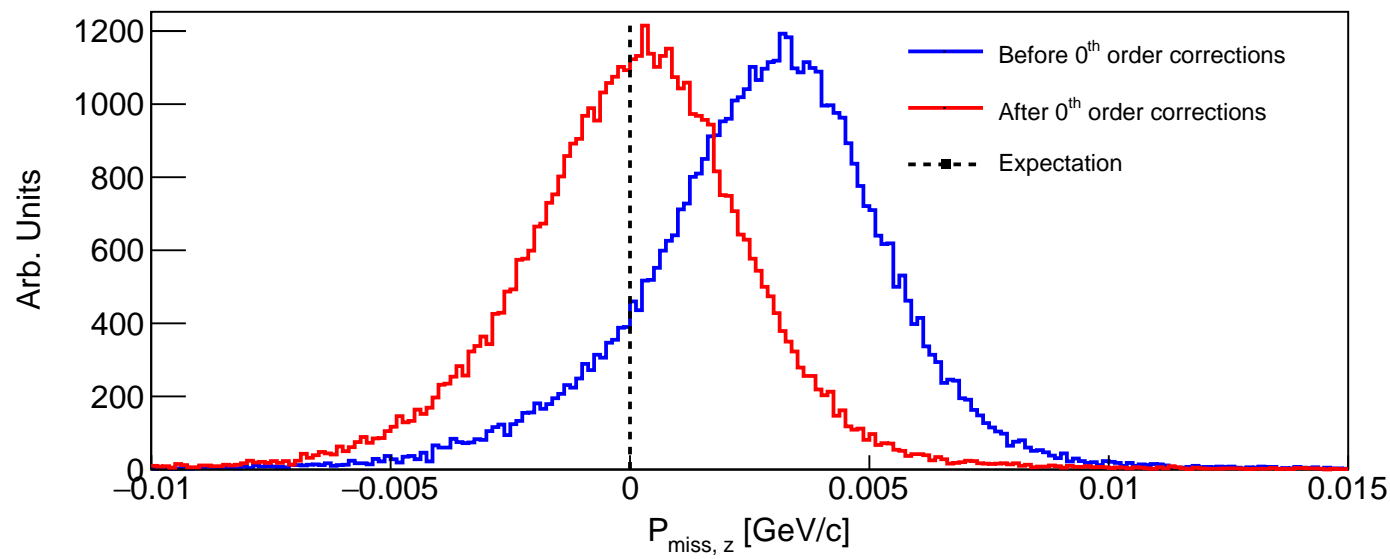

Figure A-17: Counts vs. missing momentum $z$ component. 


\section{Appendix B}

\section{${ }^{3} \mathrm{He}$ and ${ }^{3} \mathbf{H}\left(e, e^{\prime} p\right)$ kinematical distributions}

Figs. B-1 through B-5 show the measured and simulated yields for various kinematical quantities for the ${ }^{3} \mathrm{H}$, and ${ }^{3} \mathrm{He}$ targets. The simulated yields are scaled to match the measured integrated yield for each target and kinematics using scale factors of 0.60 and 0.59 (0.58 and 0.84) for the low- and high- $p_{\text {miss }}{ }^{3} \mathrm{H}\left({ }^{3} \mathrm{He}\right)$ kinematical settings respectively. These scale factors imply that the measured and simulated ${ }^{3} \mathrm{He} /{ }^{3} \mathrm{H}$ yield ratios differ by $1 \%(\approx 0.60 / 0.58)$ and $30 \%(\approx 0.59 / 0.84)$. We examined these differences for a possible $p_{\text {miss }}$ dependence and found that the data is consistent with a flat $p_{\text {miss }}$ dependence (see Fig. B-6). Specifically for the high- $p_{\text {miss }}$ kinematics, fitting the double ratio to a constant and a linear function of $p_{\text {miss }}$ gave reduced $\chi^{2}$ of 0.97 and 0.94 respectively with the resulting slope of the linear function being consistent with zero within $1 \sigma$ of its fit uncertainty.

Figs. B-7 through B-14 show correlations between different measured kinematical quantities and their comparisons to simulation.

The simulation appears to agree with the distributions of the data well enough to use for computing the corrections described in section 3.5. 

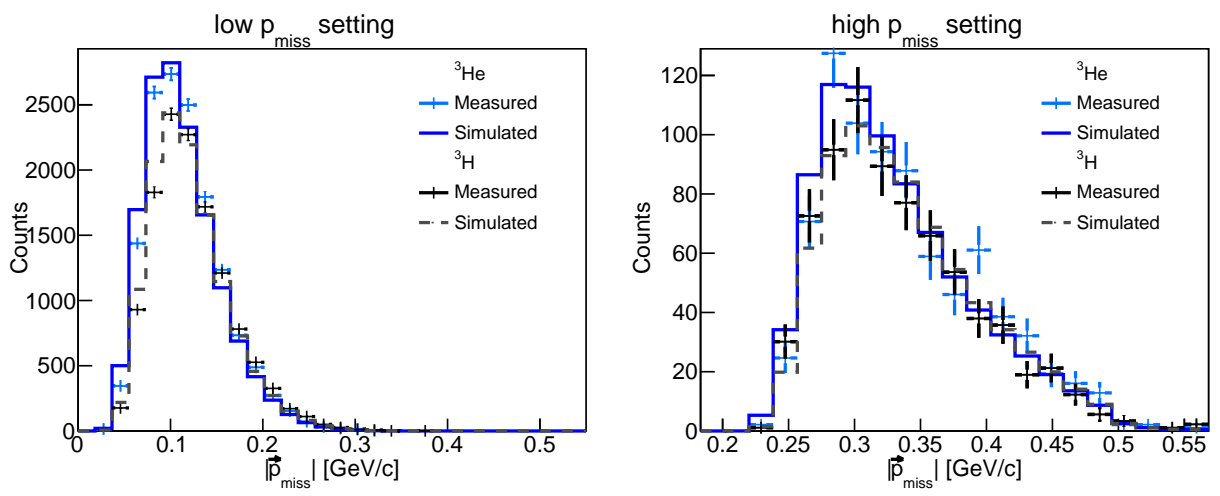

Figure B-1: Number of counts vs. $p_{\text {miss }}$ for low- $p_{\text {miss }}$ (left) and high- $p_{\text {miss }}$ (right) kinematical settings. The blue markers and lines correspond to ${ }^{3} \mathrm{He}$ measured and simulated distributions respectively. The black markers and lines correspond to ${ }^{3} \mathrm{H}$ measured and simulated distributions respectively.
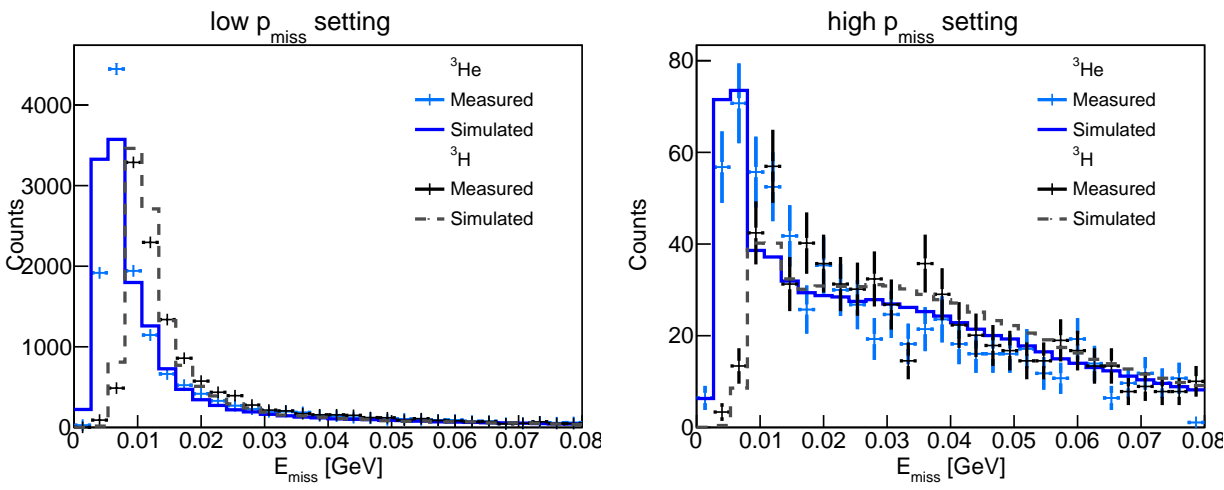

Figure B-2: Same as Fig. B-1, as a function of $E_{m i s s}$.
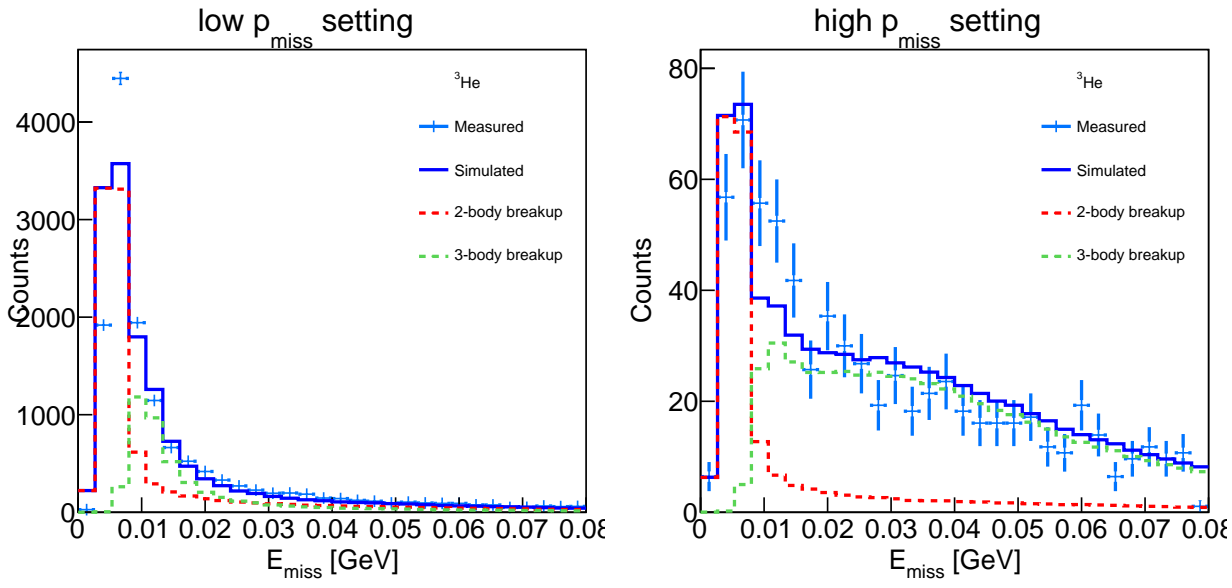

Figure B-3: Same as Fig. B-2, for ${ }^{3} \mathrm{He}$ only, with separation of the SIMC yield to contributions to $2 \mathrm{bbu}$ and $3 \mathrm{bbu}$ channels. 

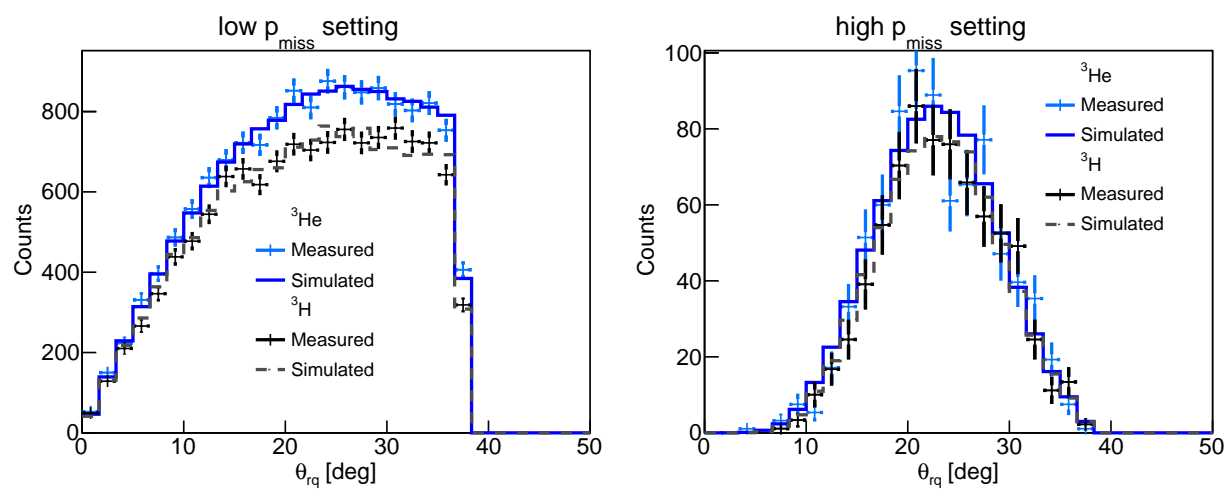

Figure B-4: Same as Fig. B-1, as a function of $\theta_{r q}$.
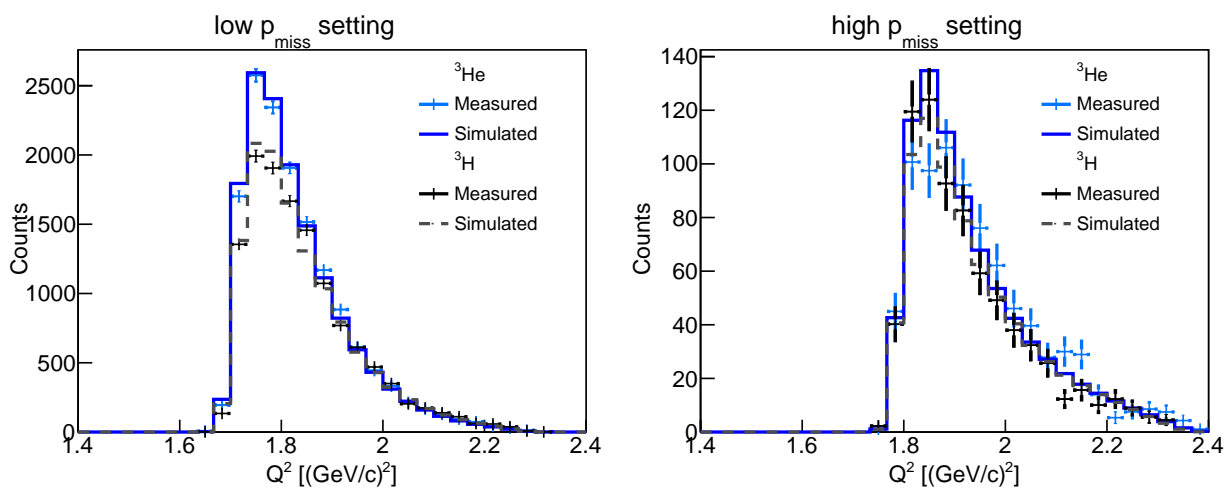

Figure B-5: Same as Fig. B-1, as a function of $Q^{2}$.

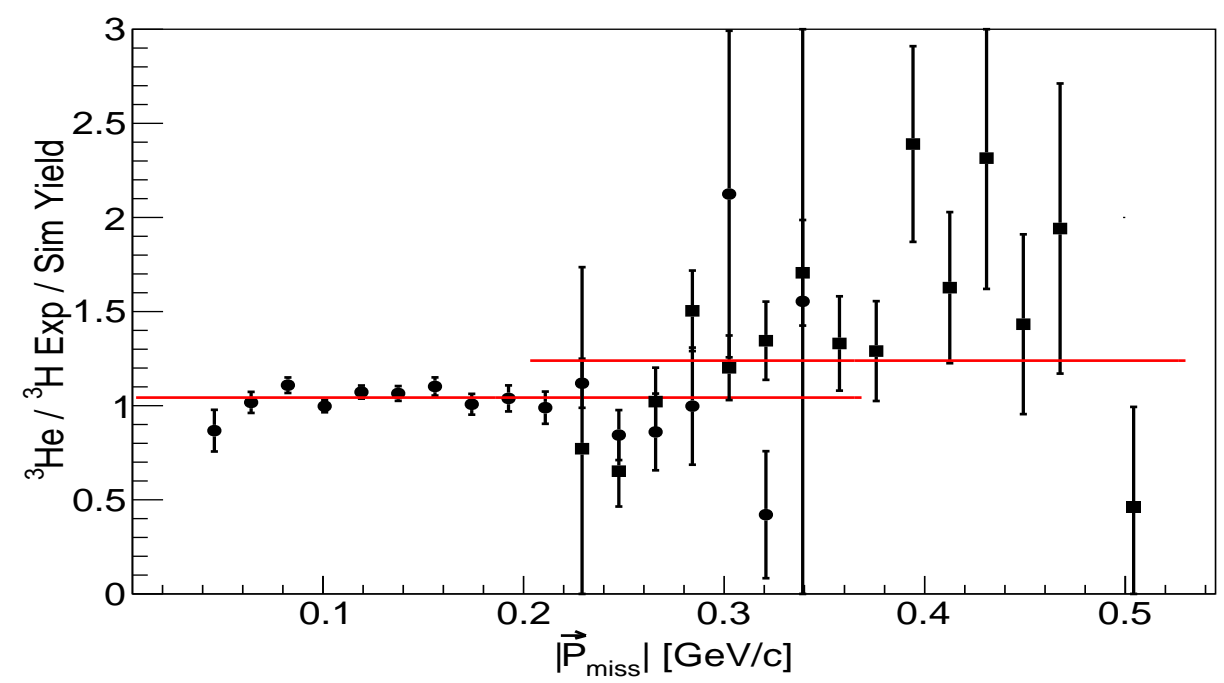

Figure B-6: $p_{\text {miss }}$ dependence of the measured over simulated ${ }^{3} \mathrm{He} /{ }^{3} \mathrm{H}$ yield ratios from Fig. B-1. The red lines are the result of fits to a constant of each kinematics. 

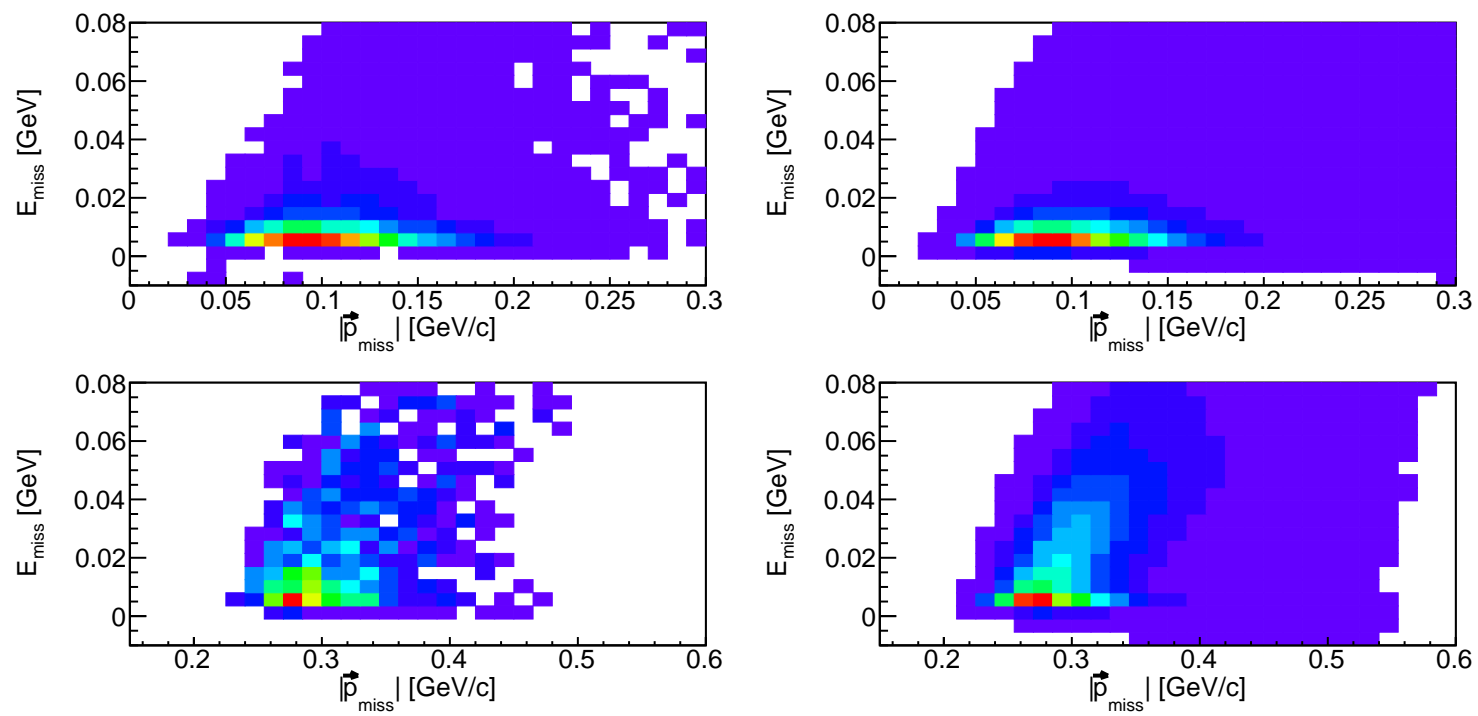

Figure B-7: ${ }^{3} \mathrm{He} E_{\text {miss }}$ vs. $p_{\text {miss }}$ measured (left column) and simulated (right column) distributions. The top and bottom rows correspond to the low- and high- $p_{m i s s}$ settings respectively.
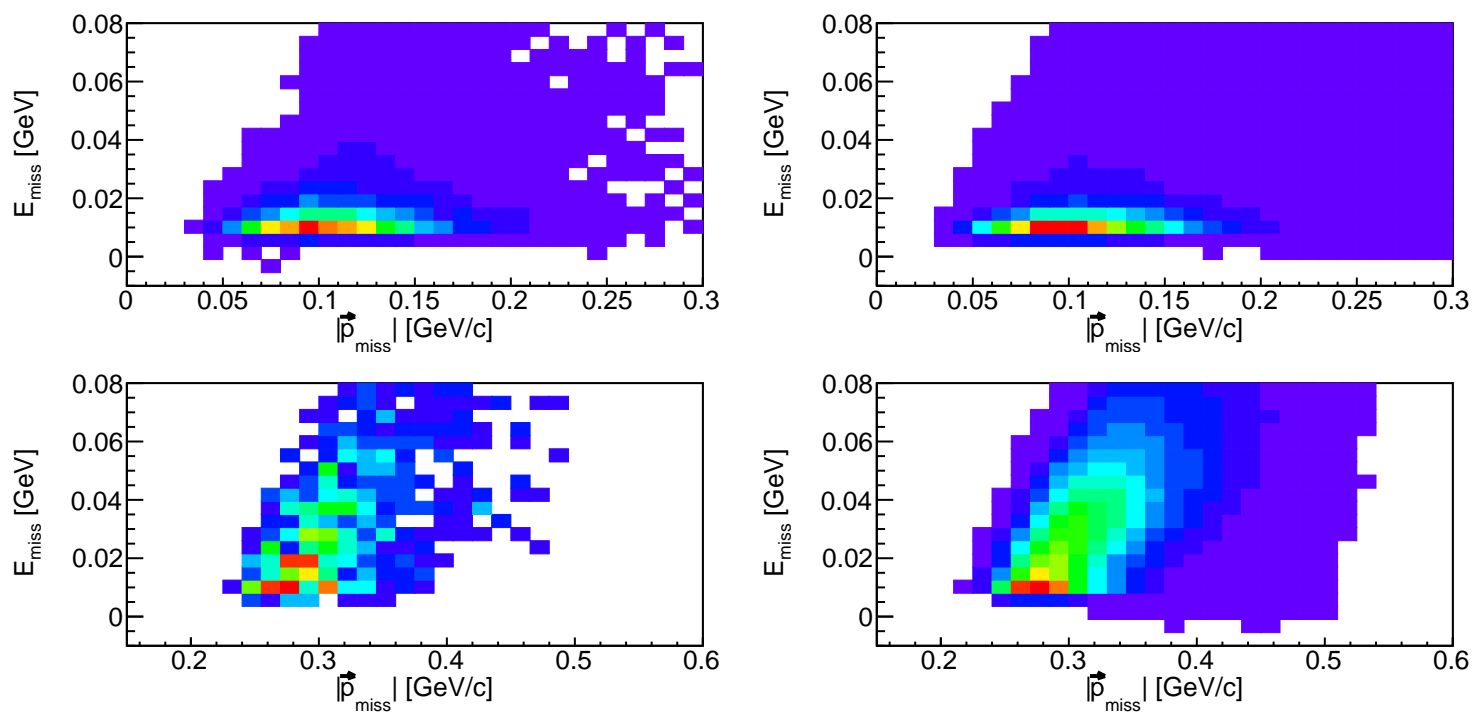

Figure B-8: Same as Fig. B-7 only for ${ }^{3} \mathrm{H}$. 

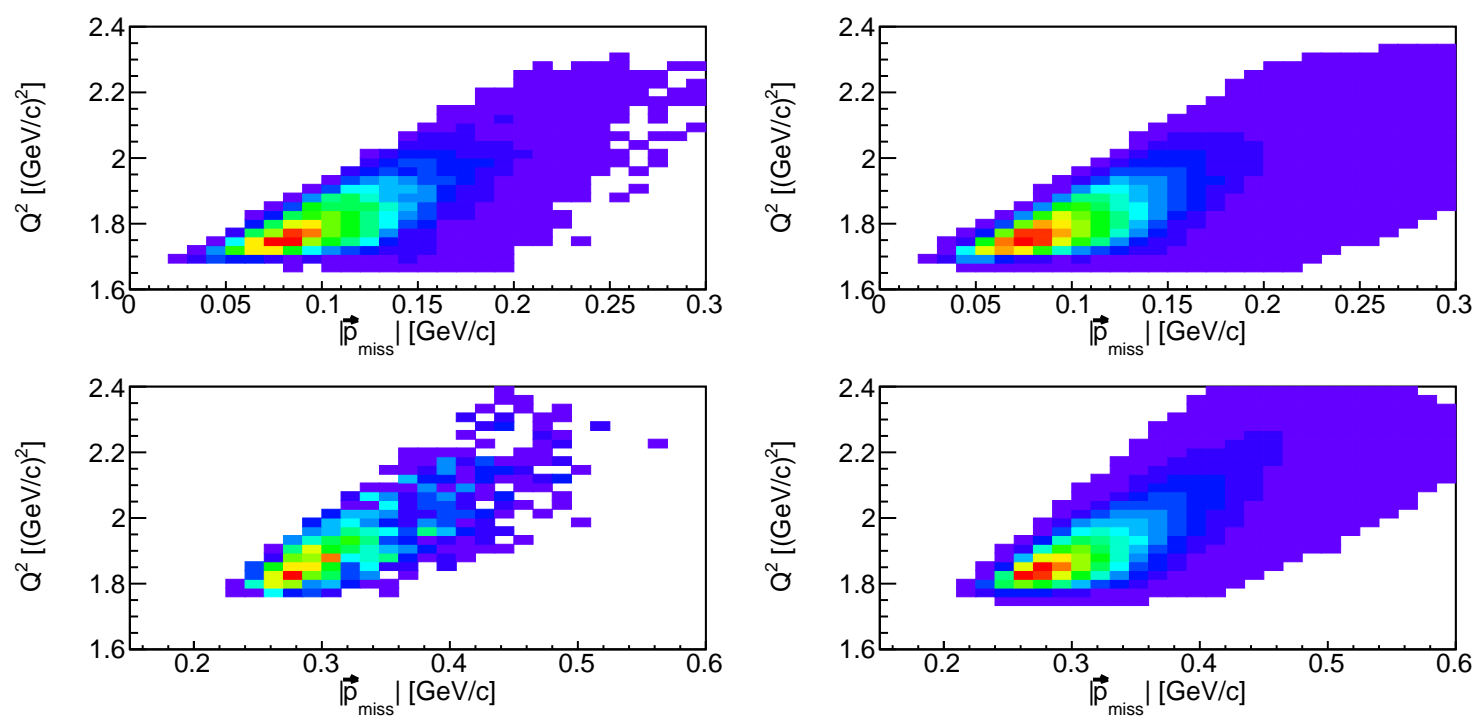

Figure B-9: ${ }^{3} \mathrm{He} Q^{2}$ vs. $p_{\text {miss }}$ measured (left column) and simulated (right column) distributions. The top and bottom rows correspond to the low- and high- $p_{\text {miss }}$ settings respectively.
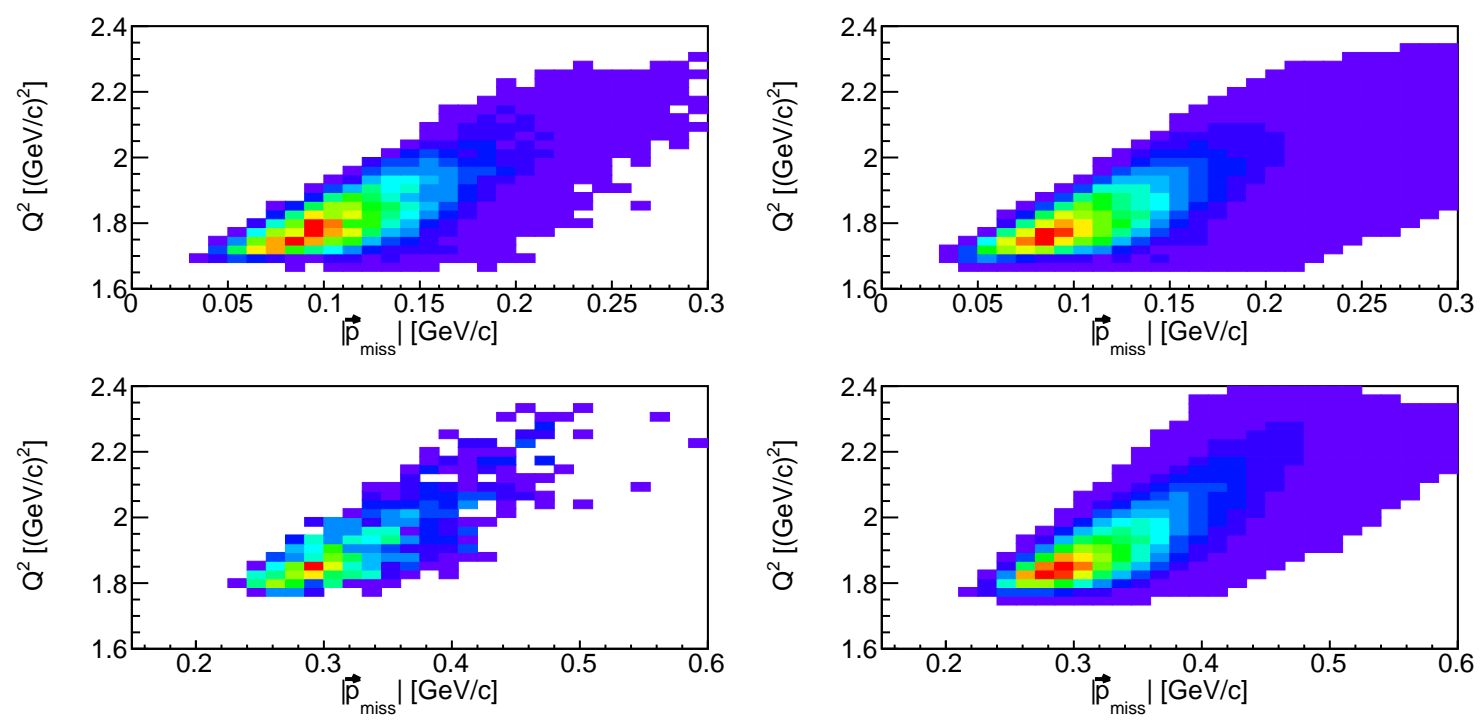

Figure B-10: Same as Fig. B-9 only for ${ }^{3} \mathrm{H}$. 

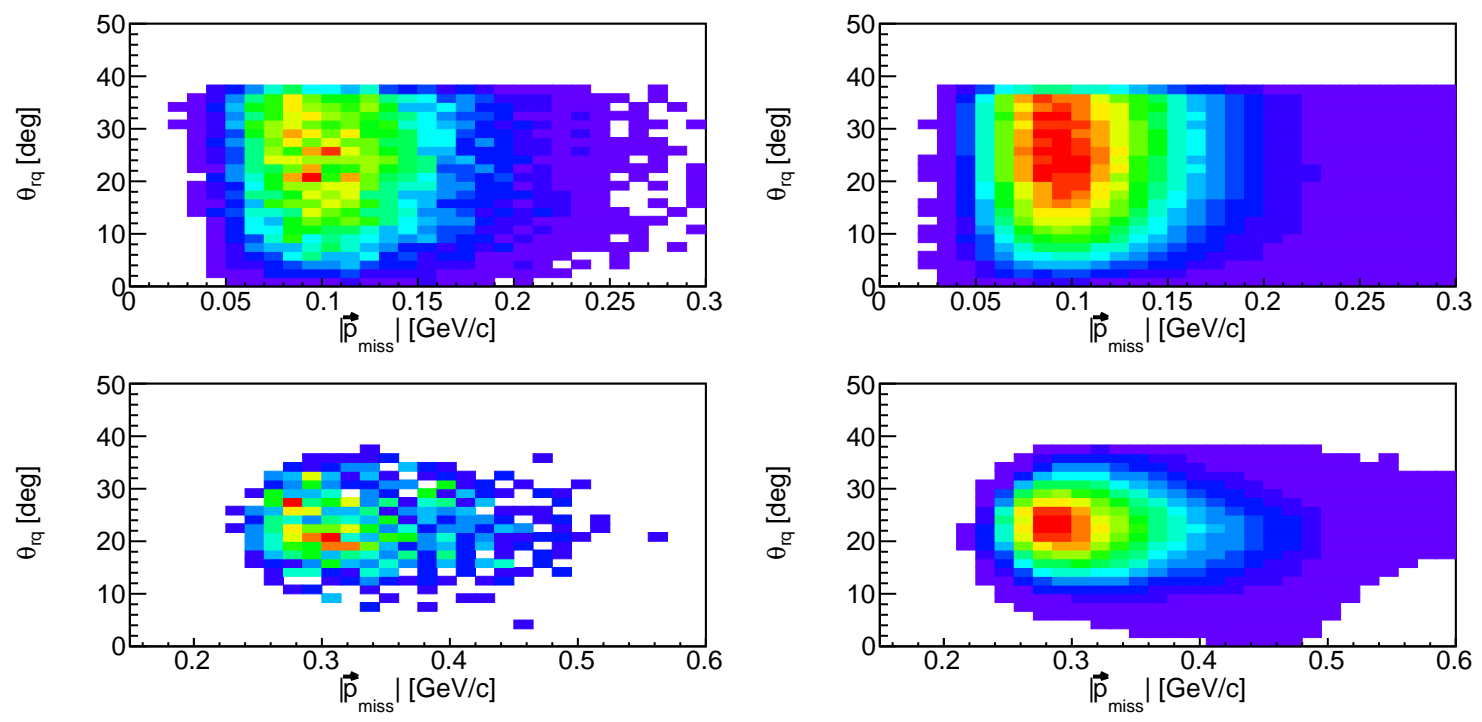

Figure B-11: ${ }^{3} \mathrm{He} \theta_{r q}$ vs. $p_{\text {miss }}$ measured (left column) and simulated (right column) distributions. The top and bottom rows correspond to the low- and high- $p_{\text {miss }}$ settings respectively.
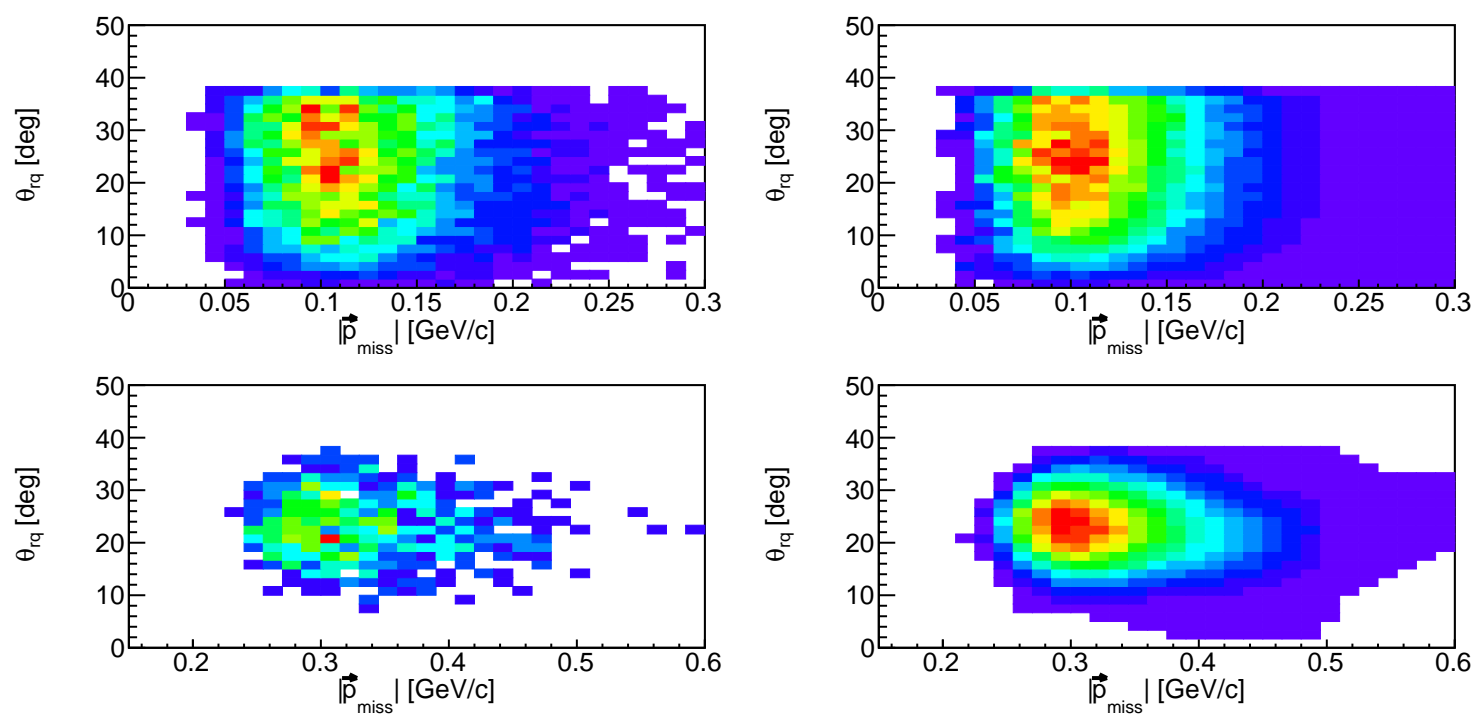

Figure B-12: Same as Fig. B-11 only for ${ }^{3} \mathrm{H}$. 

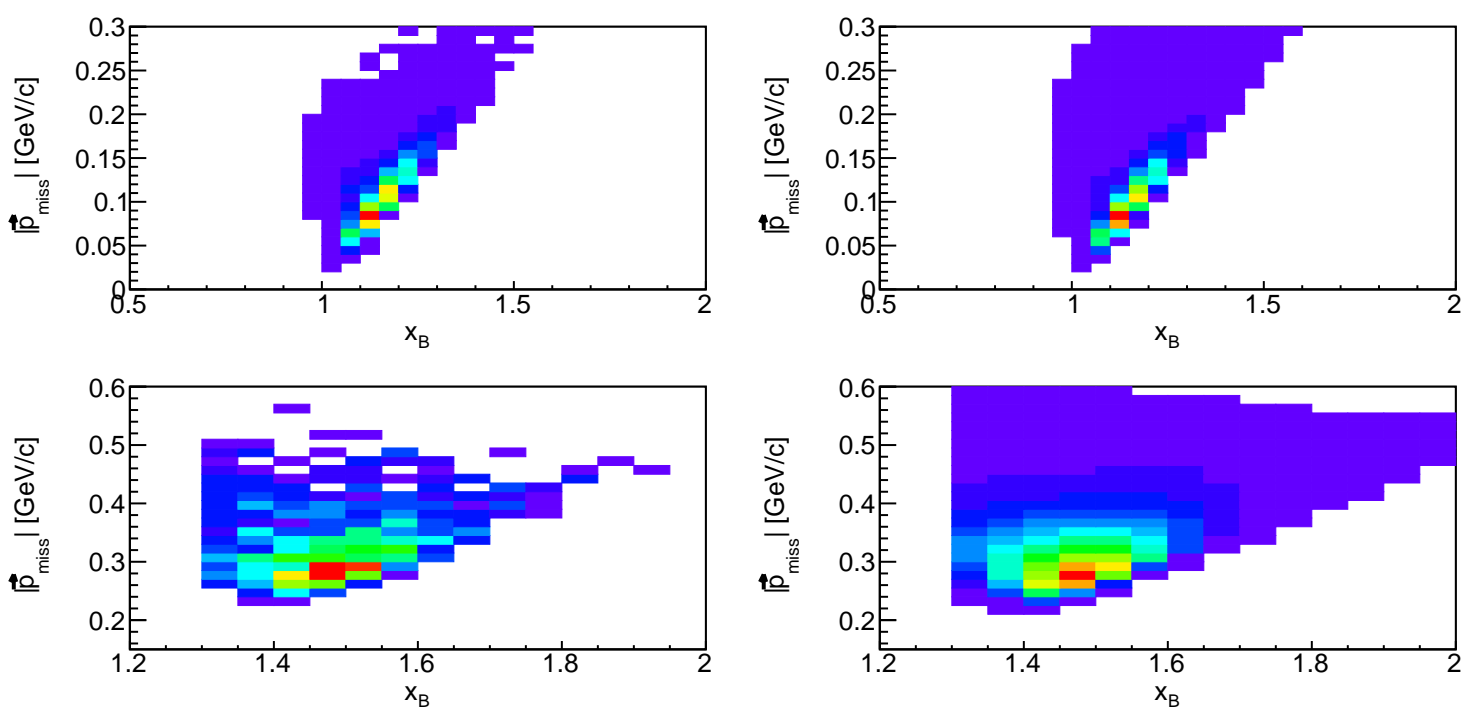

Figure B-13: ${ }^{3} \mathrm{He} p_{\text {miss }}$ vs. $x_{B}$ measured (left column) and simulated (right column) distributions. The top and bottom rows correspond to the low- and high- $p_{m i s s}$ settings respectively.
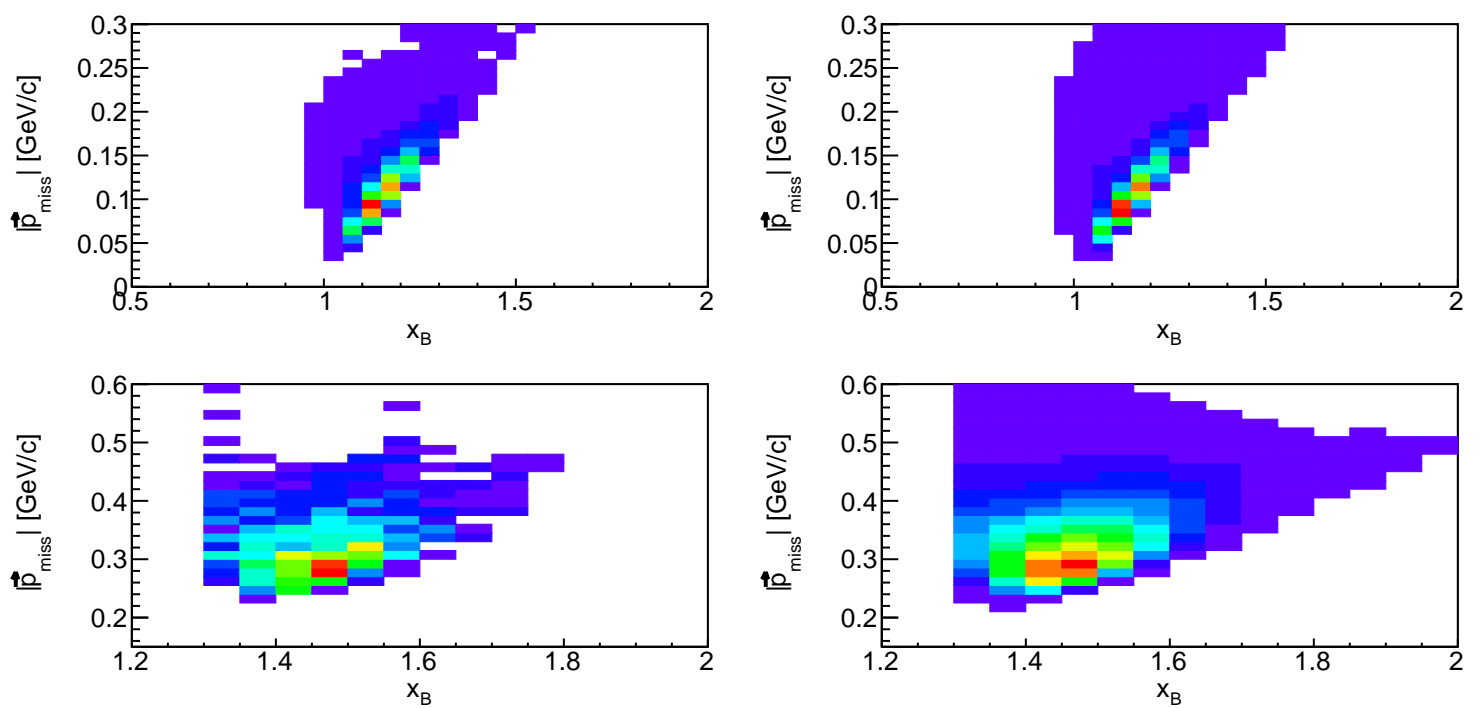

Figure B-14: Same as Fig. B-14 only for ${ }^{3} \mathrm{H}$. 


\section{Appendix C}

\section{Additional Chapter 3 Tables}

All the cross sections correspond to $\frac{d^{6} \sigma}{d E_{e} d \Omega_{e} d E_{p} d \Omega_{p}}$ and are given in units of $\frac{\mathrm{nb}}{\mathrm{MeV}^{2} \mathrm{sr}^{2}}$. Tables C.1 through C.4 correspond to the cross section values used to determine bincentering corrections in the absolute-cross-section analysis. Table C.5 corresponds to the final results from the cross-section-ratio analysis. Lastly, tables C.6 and C.7 correspond to the final results from the absolute-cross-section analysis. 
Table C.1: Acceptance-integrated cross section values and corresponding kinematical points for the low- $p_{\text {miss }}$ kinematical setting. The first and second columns correspond to the $p_{\text {miss }}[\mathrm{MeV} / c]$ and $E_{\text {miss }}[\mathrm{MeV}]$ bin ranges respectively. The third and fourth columns correspond to weighted-average $x_{B}$ and $Q^{2}\left[\left(\mathrm{GeV} / c^{2}\right)^{2}\right]$ in that $\left(p_{m i s s}, E_{m i s s}\right)$ bin respectively. The remaining columns correspond to the integrated cross section for ${ }^{3} \mathrm{He}$ and ${ }^{3} \mathrm{H}$ calculated with the $\mathrm{CK}+C C 1$ and Cracow models.

\begin{tabular}{|c|c|c|c|c|c|c|c|}
\hline \multirow{2}{*}{$p_{\text {miss }}$} & \multirow{2}{*}{$E_{m i s s}$} & \multirow{2}{*}{$x_{B}$} & \multirow{2}{*}{$Q^{2}$} & \multicolumn{2}{|c|}{${ }^{3} \mathrm{He}$} & \multicolumn{2}{|c|}{${ }^{3} \mathrm{H}$} \\
\hline & & & & $\sigma_{\mathrm{CK}+C C 1}$ & $\sigma_{\text {Cracow }}$ & $\sigma_{\mathrm{CK}+C C 1}$ & $\sigma_{\text {Cracow }}$ \\
\hline \multirow{2}{*}{$36.7-73.3$} & $8-20$ & 1.08 & 1.74 & $8.329 \mathrm{e}-02$ & 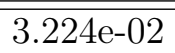 & $\overline{~ 1.674 \mathrm{e}-01}$ & $7.167 \mathrm{e}-02$ \\
\hline & $20-30$ & 1.06 & 1.72 & $2.370 \mathrm{e}-03$ & $1.976 \mathrm{e}-03$ & $3.302 \mathrm{e}-03$ & $2.673 \mathrm{e}-03$ \\
\hline \multirow{4}{*}{$73.3-91.7$} & $8-20$ & 1.12 & 1.77 & $4.632 \mathrm{e}-02$ & $1.981 \mathrm{e}-02$ & $8.612 \mathrm{e}-02$ & $4.119 \mathrm{e}-02$ \\
\hline & $20-30$ & 1.09 & 1.75 & $1.665 \mathrm{e}-03$ & $1.375 \mathrm{e}-03$ & $2.587 \mathrm{e}-03$ & $2.100 \mathrm{e}-03$ \\
\hline & $30-40$ & 1.07 & 1.74 & $3.253 \mathrm{e}-04$ & $3.035 \mathrm{e}-04$ & $4.962 \mathrm{e}-04$ & $4.529 \mathrm{e}-04$ \\
\hline & $40-50$ & 1.04 & 1.72 & $8.014 \mathrm{e}-05$ & $8.280 \mathrm{e}-05$ & $1.318 \mathrm{e}-04$ & $1.357 \mathrm{e}-04$ \\
\hline \multirow{4}{*}{$91.7-110.0$} & $8-20$ & 1.15 & 1.80 & $2.572 \mathrm{e}-02$ & $1.206 \mathrm{e}-02$ & $4.711 \mathrm{e}-02$ & $2.386 \mathrm{e}-02$ \\
\hline & $20-30$ & 1.12 & 1.79 & $1.360 \mathrm{e}-03$ & $1.099 \mathrm{e}-03$ & $2.084 \mathrm{e}-03$ & $1.662 \mathrm{e}-03$ \\
\hline & $30-40$ & 1.10 & 1.77 & $2.751 \mathrm{e}-04$ & $2.462 \mathrm{e}-04$ & $3.650 \mathrm{e}-04$ & $3.236 \mathrm{e}-04$ \\
\hline & $40-50$ & 1.07 & 1.75 & $8.430 \mathrm{e}-05$ & $8.259 \mathrm{e}-05$ & $8.832 \mathrm{e}-05$ & $8.644 \mathrm{e}-05$ \\
\hline \multirow{4}{*}{$110.0-128.3$} & $8-20$ & 1.18 & 1.84 & $1.482 \mathrm{e}-02$ & 7.661e-03 & $2.560 \mathrm{e}-02$ & $1.377 \mathrm{e}-02$ \\
\hline & $20-30$ & 1.15 & 1.82 & $1.061 \mathrm{e}-03$ & $8.468 \mathrm{e}-04$ & $1.617 \mathrm{e}-03$ & $1.274 \mathrm{e}-03$ \\
\hline & $30-40$ & 1.13 & 1.80 & $2.130 \mathrm{e}-04$ & $1.842 \mathrm{e}-04$ & $3.031 \mathrm{e}-04$ & $2.605 \mathrm{e}-04$ \\
\hline & $40-50$ & 1.10 & 1.79 & $7.212 \mathrm{e}-05$ & $6.699 \mathrm{e}-05$ & $8.331 \mathrm{e}-05$ & $7.751 \mathrm{e}-05$ \\
\hline \multirow{4}{*}{$128.3-146.7$} & $8-20$ & 1.21 & 1.88 & $8.438 \mathrm{e}-03$ & $4.678 \mathrm{e}-03$ & $1.423 \mathrm{e}-02$ & $8.167 \mathrm{e}-03$ \\
\hline & $20-30$ & 1.18 & 1.86 & $7.810 \mathrm{e}-04$ & $6.154 \mathrm{e}-04$ & $1.136 \mathrm{e}-03$ & 8.837e-04 \\
\hline & $30-40$ & 1.16 & 1.85 & $1.919 \mathrm{e}-04$ & $1.612 \mathrm{e}-04$ & $2.608 \mathrm{e}-04$ & $2.166 \mathrm{e}-04$ \\
\hline & $40-50$ & 1.13 & 1.83 & $6.414 \mathrm{e}-05$ & $5.692 \mathrm{e}-05$ & $7.241 \mathrm{e}-05$ & $6.395 \mathrm{e}-05$ \\
\hline \multirow{4}{*}{$146.7-165.0$} & $8-20$ & 1.24 & 1.93 & $4.704 \mathrm{e}-03$ & $2.781 \mathrm{e}-03$ & $7.489 \mathrm{e}-03$ & $4.556 \mathrm{e}-03$ \\
\hline & $20-30$ & 1.21 & 1.91 & $6.010 \mathrm{e}-04$ & $4.794 \mathrm{e}-04$ & 8.393e-04 & $6.485 \mathrm{e}-04$ \\
\hline & $30-40$ & 1.19 & 1.90 & $1.602 \mathrm{e}-04$ & $1.310 \mathrm{e}-04$ & $2.095 \mathrm{e}-04$ & $1.687 \mathrm{e}-04$ \\
\hline & $40-50$ & 1.16 & 1.88 & $5.500 \mathrm{e}-05$ & $4.694 \mathrm{e}-05$ & $6.931 \mathrm{e}-05$ & $5.804 \mathrm{e}-05$ \\
\hline \multirow{4}{*}{$165.0-183.3$} & $8-20$ & 1.27 & 1.98 & $2.589 \mathrm{e}-03$ & $1.622 \mathrm{e}-03$ & $4.012 \mathrm{e}-03$ & $2.564 \mathrm{e}-03$ \\
\hline & $20-30$ & 1.24 & 1.96 & $4.471 \mathrm{e}-04$ & $3.502 \mathrm{e}-04$ & $5.961 \mathrm{e}-04$ & $4.615 \mathrm{e}-04$ \\
\hline & $30-40$ & 1.22 & 1.95 & $1.336 \mathrm{e}-04$ & $1.074 \mathrm{e}-04$ & $1.674 \mathrm{e}-04$ & $1.320 \mathrm{e}-04$ \\
\hline & $40-50$ & 1.19 & 1.93 & $4.845 \mathrm{e}-05$ & $3.998 \mathrm{e}-05$ & $5.581 \mathrm{e}-05$ & $4.455 \mathrm{e}-05$ \\
\hline \multirow{4}{*}{$183.3-201.7$} & $8-20$ & 1.30 & 2.03 & $1.418 \mathrm{e}-03$ & $9.436 \mathrm{e}-04$ & $2.088 \mathrm{e}-03$ & $1.414 \mathrm{e}-03$ \\
\hline & $20-30$ & 1.27 & 2.01 & $3.126 \mathrm{e}-04$ & $2.466 \mathrm{e}-04$ & $4.167 \mathrm{e}-04$ & $3.243 \mathrm{e}-04$ \\
\hline & $30-40$ & 1.24 & 2.00 & $1.072 \mathrm{e}-04$ & $8.507 \mathrm{e}-05$ & $1.311 \mathrm{e}-04$ & $1.018 \mathrm{e}-04$ \\
\hline & $40-50$ & 1.22 & 1.98 & $4.074 \mathrm{e}-05$ & $3.272 \mathrm{e}-05$ & $5.046 \mathrm{e}-05$ & $3.893 \mathrm{e}-05$ \\
\hline \multirow{4}{*}{$201.7-220.0$} & $8-20$ & 1.33 & 2.08 & $7.642 \mathrm{e}-04$ & $5.418 \mathrm{e}-04$ & $1.095 \mathrm{e}-03$ & $7.808 \mathrm{e}-04$ \\
\hline & $20-30$ & 1.30 & 2.06 & $2.209 \mathrm{e}-04$ & $1.765 \mathrm{e}-04$ & $2.811 \mathrm{e}-04$ & $2.212 \mathrm{e}-04$ \\
\hline & $30-40$ & 1.27 & 2.05 & $8.217 \mathrm{e}-05$ & $6.484 \mathrm{e}-05$ & $1.021 \mathrm{e}-04$ & 7.861e-05 \\
\hline & $40-50$ & 1.25 & 2.03 & $3.505 \mathrm{e}-05$ & $2.759 \mathrm{e}-05$ & $4.258 \mathrm{e}-05$ & $3.211 \mathrm{e}-05$ \\
\hline \multirow{4}{*}{$220.0-256.7$} & $8-20$ & 1.36 & 2.13 & $3.271 \mathrm{e}-04$ & $2.489 \mathrm{e}-04$ & $4.442 \mathrm{e}-04$ & $3.379 \mathrm{e}-04$ \\
\hline & $20-30$ & 1.34 & 2.12 & $1.268 \mathrm{e}-04$ & $1.039 \mathrm{e}-04$ & $1.582 \mathrm{e}-04$ & $1.269 \mathrm{e}-04$ \\
\hline & $30-40$ & 1.31 & 2.11 & $5.801 \mathrm{e}-05$ & $4.548 \mathrm{e}-05$ & $7.159 \mathrm{e}-05$ & $5.456 \mathrm{e}-05$ \\
\hline & $40-50$ & 1.29 & 2.10 & $2.640 \mathrm{e}-05$ & $2.037 \mathrm{e}-05$ & $3.248 \mathrm{e}-05$ & $2.397 \mathrm{e}-05$ \\
\hline
\end{tabular}




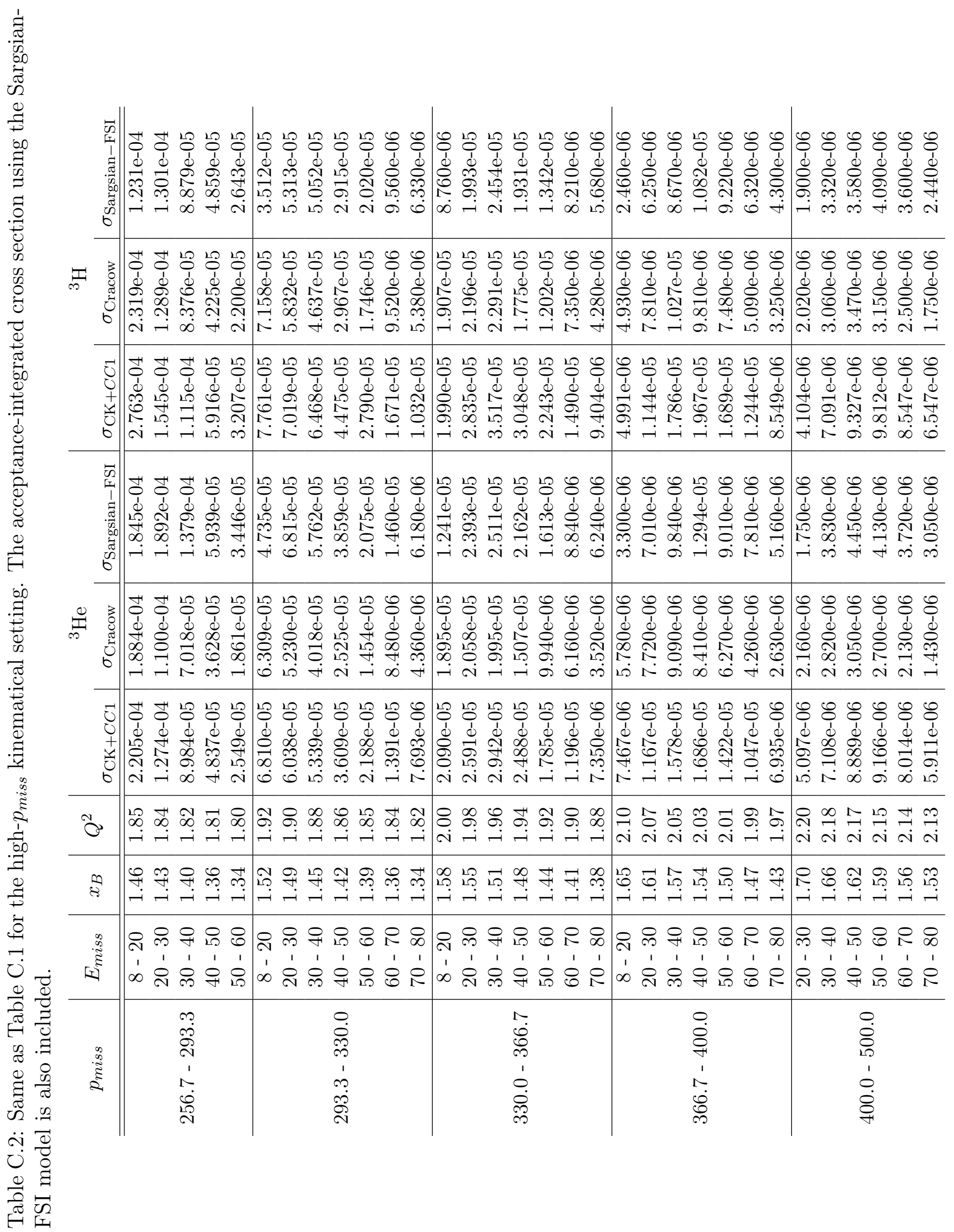


Table C.3: Point cross section values and corresponding kinematical points for the low- $p_{\text {miss }}$ kinematical setting. The first and second columns correspond to the $p_{\text {miss }}$ $[\mathrm{MeV} / c]$ and $E_{\text {miss }}[\mathrm{MeV}]$ values respectively, evaluated at the bin center. The third and fourth columns correspond to weighted-average $x_{B}$ and $Q^{2}\left[\left(\mathrm{GeV} / c^{2}\right)^{2}\right]$ in that $\left(p_{\text {miss }}, E_{\text {miss }}\right)$ bin respectively. The out-of-plane angles for the electron and proton are always fixed at $\phi_{e^{\prime}}=0^{\circ}$ and $\phi_{p}=180^{\circ}$. The remaining columns correspond to the point cross section for ${ }^{3} \mathrm{He}$ and ${ }^{3} \mathrm{H}$ calculated with the $\mathrm{CK}+C C 1$ and Cracow models.

\begin{tabular}{|c|c|c|c|c|c|c|c|}
\hline \multirow{2}{*}{$p_{m i s s}$} & \multirow{2}{*}{$E_{\text {miss }}$} & \multirow{2}{*}{$x_{B}$} & \multirow{2}{*}{$Q^{2}$} & \multicolumn{2}{|c|}{${ }^{3} \mathrm{He}$} & \multicolumn{2}{|c|}{${ }^{3} \mathrm{H}$} \\
\hline & & & & $\sigma_{\mathrm{CK}+C C 1}$ & $\sigma_{\text {Cracow }}$ & $\sigma_{\mathrm{CK}+C C 1}$ & $\sigma_{\text {Cracow }}$ \\
\hline \multirow{2}{*}{65.0} & 14 & 1.08 & 1.74 & $3.277 \mathrm{e}-02$ & $1.797 \mathrm{e}-02$ & $5.270 \mathrm{e}-02$ & $3.547 \mathrm{e}-02$ \\
\hline & 25 & 1.06 & 1.72 & $1.642 \mathrm{e}-03$ & $1.307 \mathrm{e}-03$ & $2.503 \mathrm{e}-03$ & $2.229 \mathrm{e}-03$ \\
\hline \multirow{4}{*}{82.5} & 14 & 1.12 & 1.77 & $2.375 \mathrm{e}-02$ & $1.333 \mathrm{e}-02$ & $3.731 \mathrm{e}-02$ & $2.539 \mathrm{e}-02$ \\
\hline & 25 & 1.09 & 1.75 & $1.439 \mathrm{e}-03$ & $1.116 \mathrm{e}-03$ & $2.128 \mathrm{e}-03$ & $1.863 \mathrm{e}-03$ \\
\hline & 35 & 1.07 & 1.74 & $2.695 \mathrm{e}-04$ & $2.350 \mathrm{e}-04$ & $3.714 \mathrm{e}-04$ & $3.475 \mathrm{e}-04$ \\
\hline & 45 & 1.04 & 1.72 & $7.805 \mathrm{e}-05$ & 7.643e-05 & $8.745 \mathrm{e}-05$ & $9.245 \mathrm{e}-05$ \\
\hline \multirow{4}{*}{100.8} & 14 & 1.15 & 1.80 & $1.672 \mathrm{e}-02$ & $9.678 \mathrm{e}-03$ & $2.560 \mathrm{e}-02$ & $1.768 \mathrm{e}-02$ \\
\hline & 25 & 1.12 & 1.79 & $1.216 \mathrm{e}-03$ & $9.191 \mathrm{e}-04$ & $1.734 \mathrm{e}-03$ & $1.481 \mathrm{e}-03$ \\
\hline & 35 & 1.10 & 1.77 & $2.520 \mathrm{e}-04$ & $2.091 \mathrm{e}-04$ & $3.385 \mathrm{e}-04$ & $3.061 \mathrm{e}-04$ \\
\hline & 45 & 1.07 & 1.75 & $7.584 \mathrm{e}-05$ & $7.007 \mathrm{e}-05$ & $8.649 \mathrm{e}-05$ & $8.575 \mathrm{e}-05$ \\
\hline \multirow{4}{*}{119.2} & 14 & 1.18 & 1.84 & $1.100 \mathrm{e}-02$ & $6.616 \mathrm{e}-03$ & $1.637 \mathrm{e}-02$ & $1.156 \mathrm{e}-02$ \\
\hline & 25 & 1.15 & 1.82 & $1.027 \mathrm{e}-03$ & $7.620 \mathrm{e}-04$ & $1.409 \mathrm{e}-03$ & $1.174 \mathrm{e}-03$ \\
\hline & 35 & 1.13 & 1.80 & $2.321 \mathrm{e}-04$ & $1.855 \mathrm{e}-04$ & $3.029 \mathrm{e}-04$ & $2.637 \mathrm{e}-04$ \\
\hline & 45 & 1.10 & 1.79 & $7.120 \mathrm{e}-05$ & $6.214 \mathrm{e}-05$ & $8.191 \mathrm{e}-05$ & $7.691 \mathrm{e}-05$ \\
\hline \multirow{4}{*}{137.5} & 14 & 1.21 & 1.88 & $7.074 \mathrm{e}-03$ & $4.416 \mathrm{e}-03$ & $1.026 \mathrm{e}-02$ & 7.373e-03 \\
\hline & 25 & 1.18 & 1.86 & $8.358 \mathrm{e}-04$ & $6.117 \mathrm{e}-04$ & $1.107 \mathrm{e}-03$ & $9.011 \mathrm{e}-04$ \\
\hline & 35 & 1.16 & 1.85 & $2.015 \mathrm{e}-04$ & $1.562 \mathrm{e}-04$ & $2.559 \mathrm{e}-04$ & $2.143 \mathrm{e}-04$ \\
\hline & 45 & 1.13 & 1.83 & $6.611 \mathrm{e}-05$ & $5.513 \mathrm{e}-05$ & 7.637e-05 & $6.799 \mathrm{e}-05$ \\
\hline \multirow{4}{*}{155.8} & 14 & 1.24 & 1.93 & $4.301 \mathrm{e}-03$ & $2.805 \mathrm{e}-03$ & $6.075 \mathrm{e}-03$ & $4.475 \mathrm{e}-03$ \\
\hline & 25 & 1.21 & 1.91 & $6.519 \mathrm{e}-04$ & $4.738 \mathrm{e}-04$ & 8.362e-04 & 6.702e-04 \\
\hline & 35 & 1.19 & 1.90 & $1.729 \mathrm{e}-04$ & $1.308 \mathrm{e}-04$ & $2.140 \mathrm{e}-04$ & $1.735 \mathrm{e}-04$ \\
\hline & 45 & 1.16 & 1.88 & $5.936 \mathrm{e}-05$ & $4.761 \mathrm{e}-05$ & $6.860 \mathrm{e}-05$ & $5.774 \mathrm{e}-05$ \\
\hline \multirow{4}{*}{174.2} & 14 & 1.27 & 1.98 & $2.542 \mathrm{e}-03$ & $1.742 \mathrm{e}-03$ & $3.498 \mathrm{e}-03$ & $2.657 \mathrm{e}-03$ \\
\hline & 25 & 1.24 & 1.96 & $4.965 \mathrm{e}-04$ & $3.615 \mathrm{e}-04$ & $6.198 \mathrm{e}-04$ & $4.932 \mathrm{e}-04$ \\
\hline & 35 & 1.22 & 1.95 & $1.459 \mathrm{e}-04$ & $1.086 \mathrm{e}-04$ & $1.770 \mathrm{e}-04$ & $1.400 \mathrm{e}-04$ \\
\hline & 45 & 1.19 & 1.93 & $5.274 \mathrm{e}-05$ & $4.095 \mathrm{e}-05$ & $6.099 \mathrm{e}-05$ & $4.885 \mathrm{e}-05$ \\
\hline \multirow{4}{*}{192.5} & 14 & 1.30 & 2.03 & $1.461 \mathrm{e}-03$ & $1.058 \mathrm{e}-03$ & $1.958 \mathrm{e}-03$ & $1.548 \mathrm{e}-03$ \\
\hline & 25 & 1.27 & 2.01 & $3.687 \mathrm{e}-04$ & $2.712 \mathrm{e}-04$ & $4.505 \mathrm{e}-04$ & $3.580 \mathrm{e}-04$ \\
\hline & 35 & 1.24 & 2.00 & $1.230 \mathrm{e}-04$ & $9.065 \mathrm{e}-05$ & $1.470 \mathrm{e}-04$ & $1.144 \mathrm{e}-04$ \\
\hline & 45 & 1.22 & 1.98 & $4.632 \mathrm{e}-05$ & $3.504 \mathrm{e}-05$ & $5.369 \mathrm{e}-05$ & $4.150 \mathrm{e}-05$ \\
\hline \multirow{4}{*}{210.8} & 14 & 1.33 & 2.08 & $8.181 \mathrm{e}-04$ & $6.305 \mathrm{e}-04$ & $1.067 \mathrm{e}-03$ & 8.853e-04 \\
\hline & 25 & 1.30 & 2.06 & $2.658 \mathrm{e}-04$ & $1.994 \mathrm{e}-04$ & $3.195 \mathrm{e}-04$ & $2.555 \mathrm{e}-04$ \\
\hline & 35 & 1.27 & 2.05 & $1.004 \mathrm{e}-04$ & $7.377 \mathrm{e}-05$ & $1.188 \mathrm{e}-04$ & $9.164 \mathrm{e}-05$ \\
\hline & 45 & 1.25 & 2.03 & $4.019 \mathrm{e}-05$ & $2.983 \mathrm{e}-05$ & $4.677 \mathrm{e}-05$ & $3.533 \mathrm{e}-05$ \\
\hline \multirow{4}{*}{238.3} & 14 & 1.36 & 2.13 & $3.686 \mathrm{e}-04$ & $2.999 \mathrm{e}-04$ & $4.630 \mathrm{e}-04$ & $3.965 \mathrm{e}-04$ \\
\hline & 25 & 1.34 & 2.12 & $1.604 \mathrm{e}-04$ & $1.240 \mathrm{e}-04$ & $1.900 \mathrm{e}-04$ & $1.529 \mathrm{e}-04$ \\
\hline & 35 & 1.31 & 2.11 & $7.422 \mathrm{e}-05$ & $5.450 \mathrm{e}-05$ & $8.740 \mathrm{e}-05$ & $6.652 \mathrm{e}-05$ \\
\hline & 45 & 1.29 & 2.10 & $3.236 \mathrm{e}-05$ & $2.362 \mathrm{e}-05$ & $3.806 \mathrm{e}-05$ & $2.812 \mathrm{e}-05$ \\
\hline
\end{tabular}




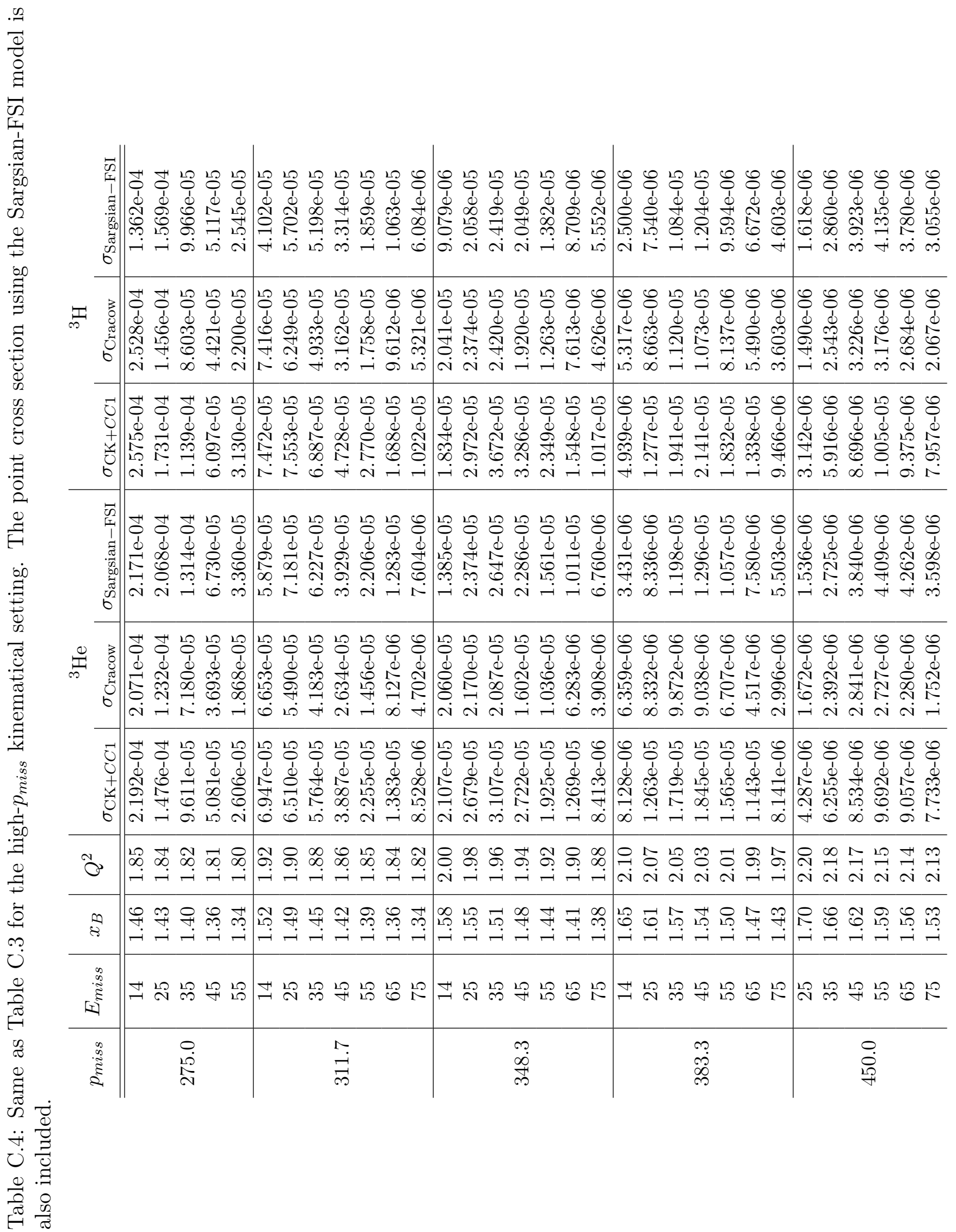



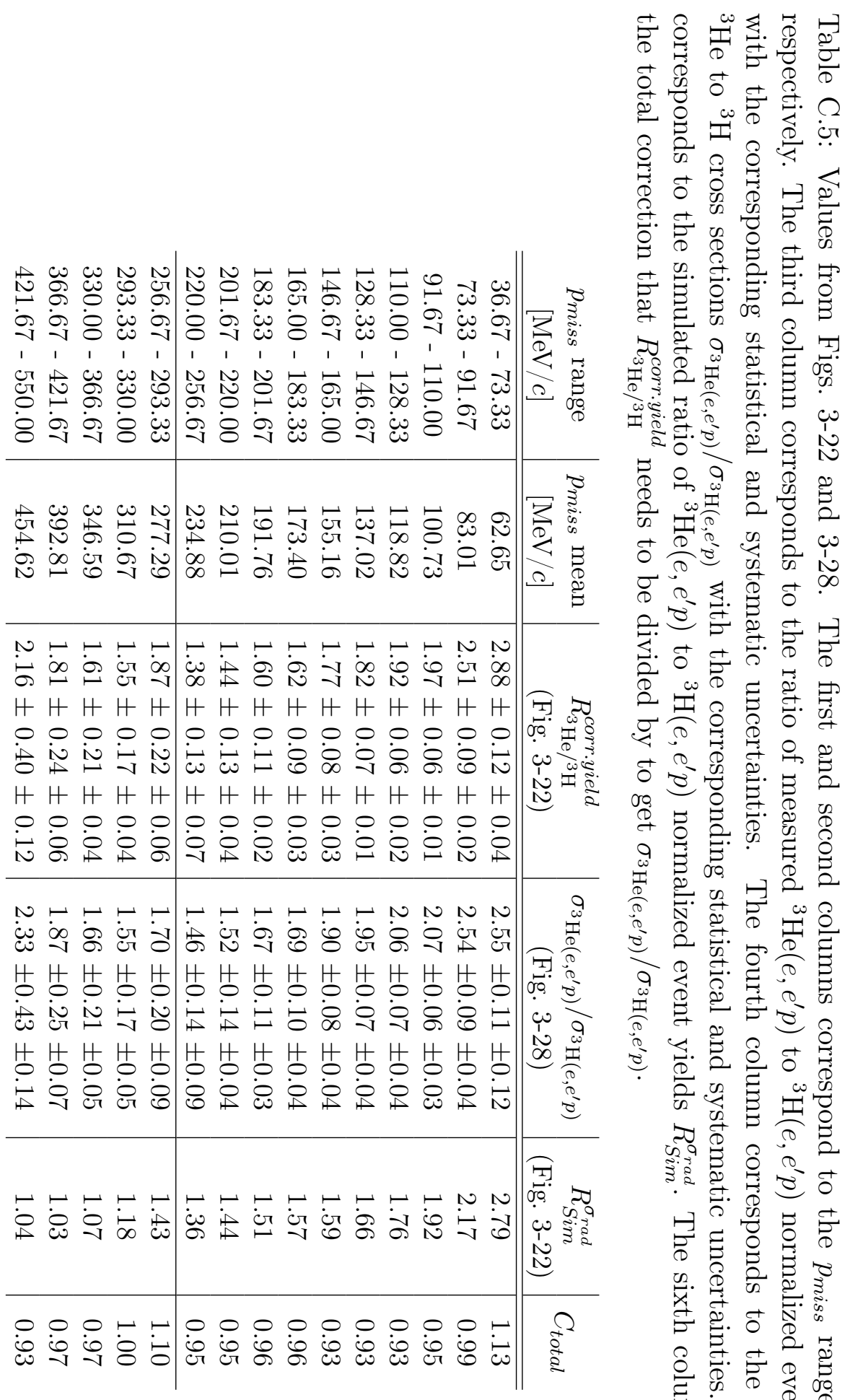
索泀. 志

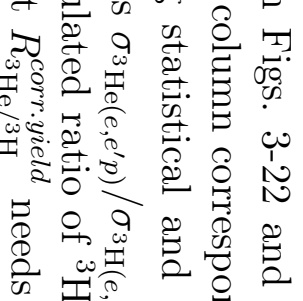

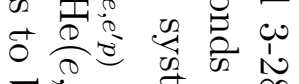
D 0.3.

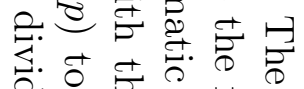

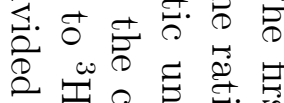
ट

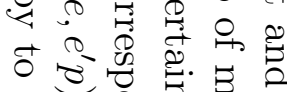

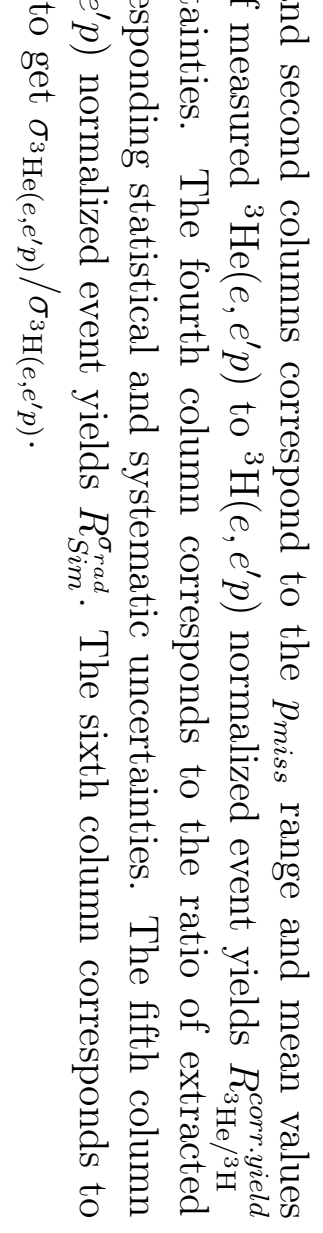




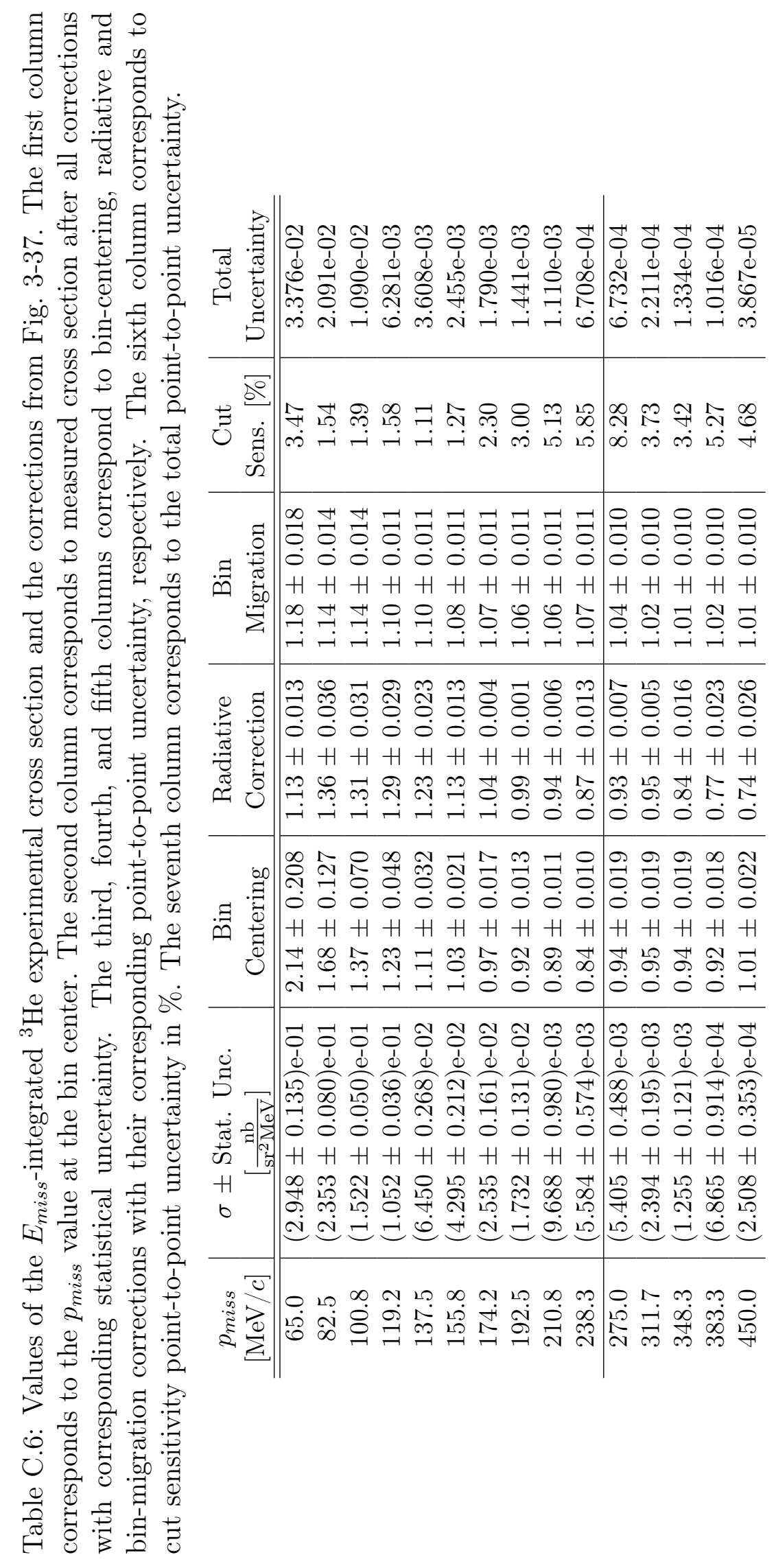




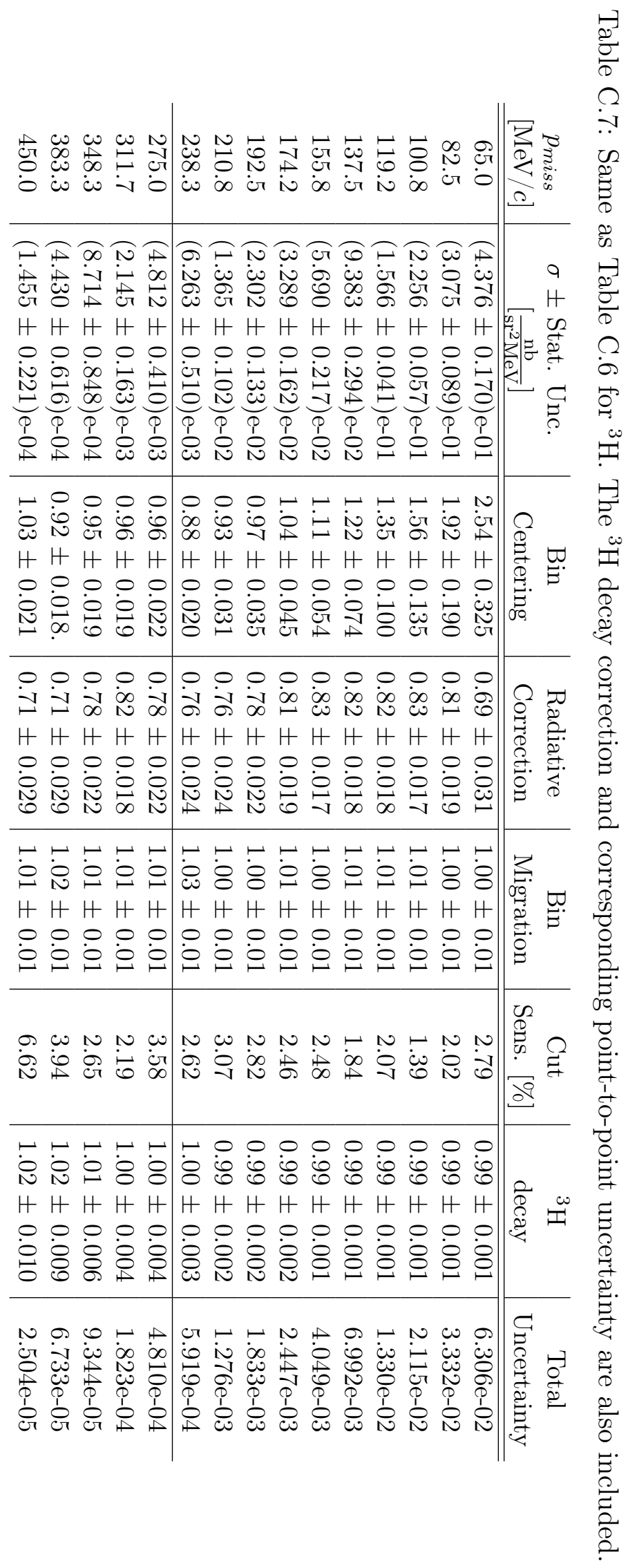




\section{Appendix D}

\section{Generalized Contact Formalism}

\section{1-body momentum distributions}

Comparison between the 1-body momentum distributions from VMC (black solid lines) and the corresponding GCF distributions (red solid lines) determined from contacts extracted in momentum- and coordinate-spaces. The contributions from different contact channels are shown as dashed lines (the sum of the dashed lines equals the solid red line). The lower panel in each figure shows the ratio of the GCF over the VMC 1-body momentum distributions. The gray band shows the $10 \%$ level of agreement. The width of the red band corresponds to the uncertainty from the contacts, and the width of the black band of value 1 corresponds to the uncertainty from the VMC calculation. 


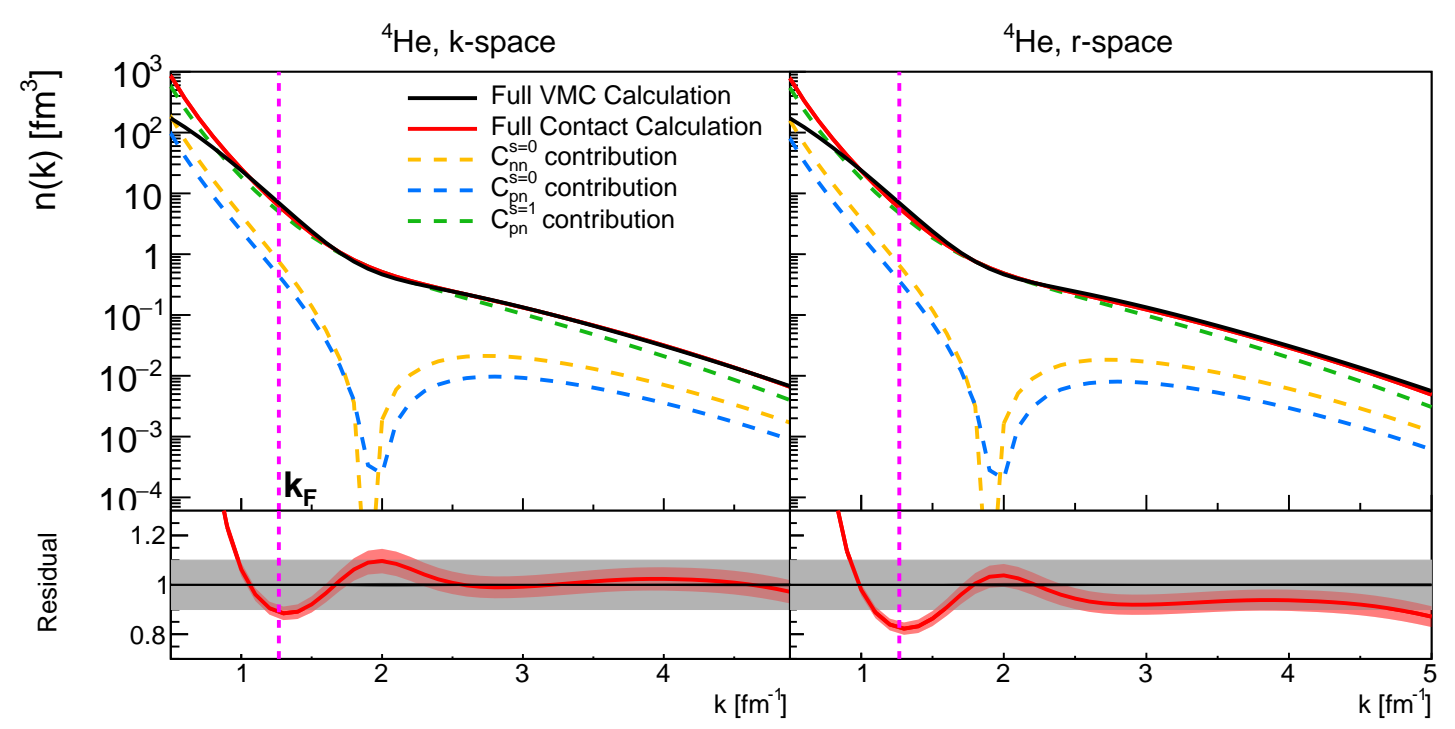

Figure D-1: Comparison between the ${ }^{4} \mathrm{He}$ 1-body momentum distributions from VMC and the corresponding GCF distributions determined from contacts extracted in momentum- (left) and coordinate- (right) spaces.

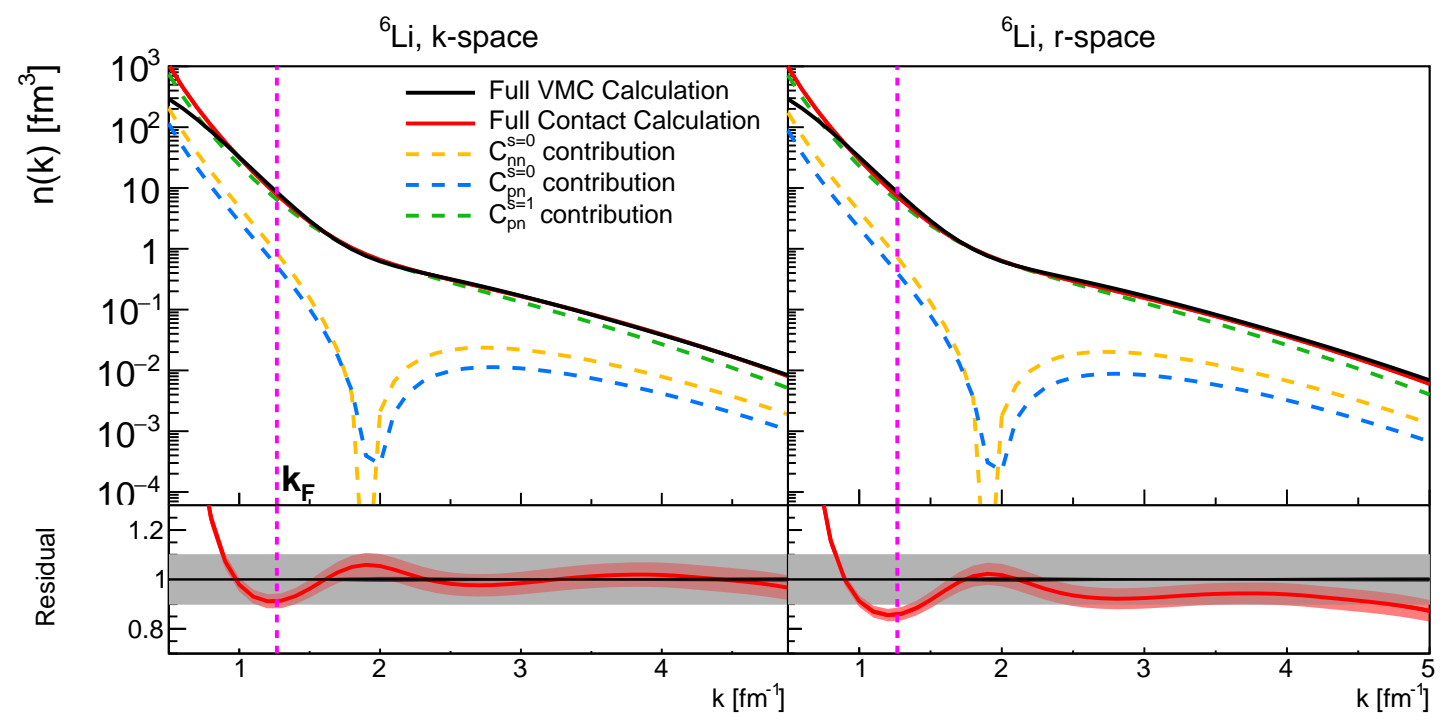

Figure D-2: Same as Fig. D-1, in the case of ${ }^{6} \mathrm{Li}$. 


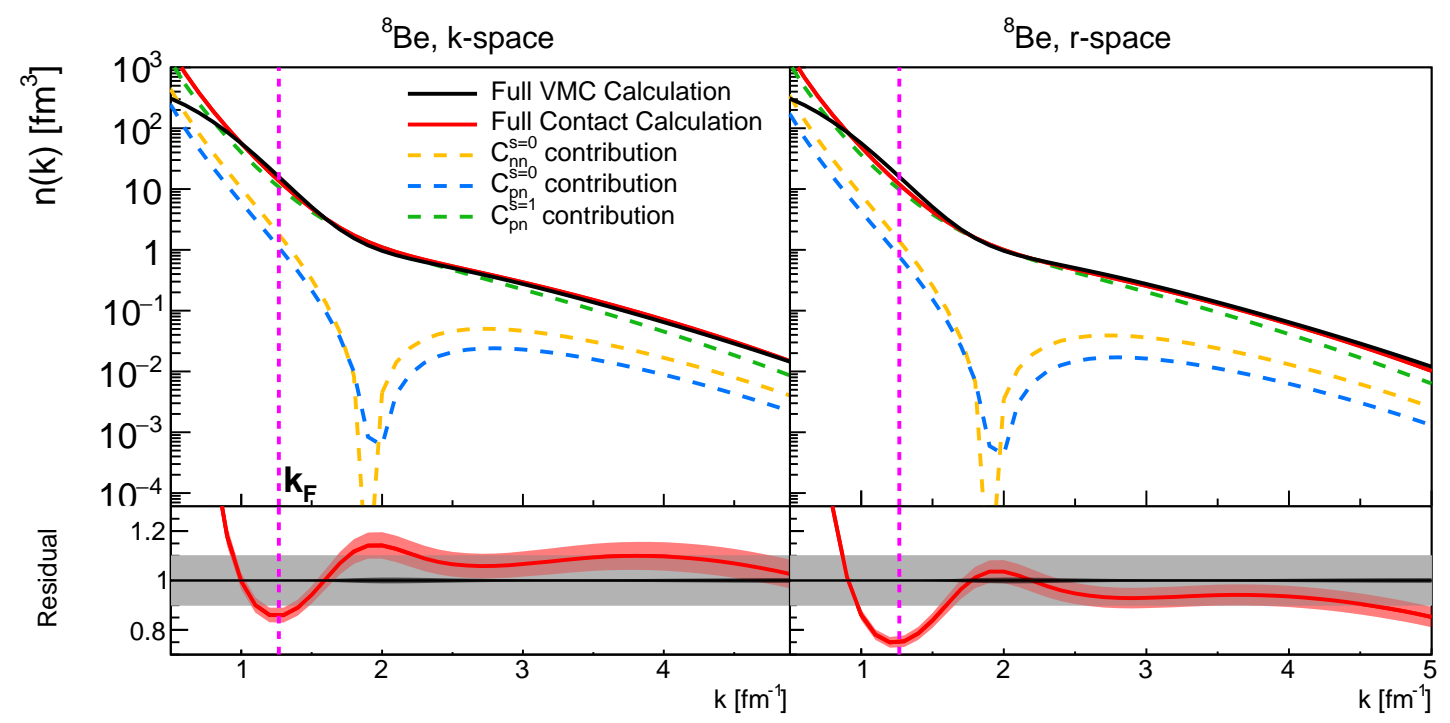

Figure D-3: Same as Fig. D-1, in the case of ${ }^{8}$ Be.

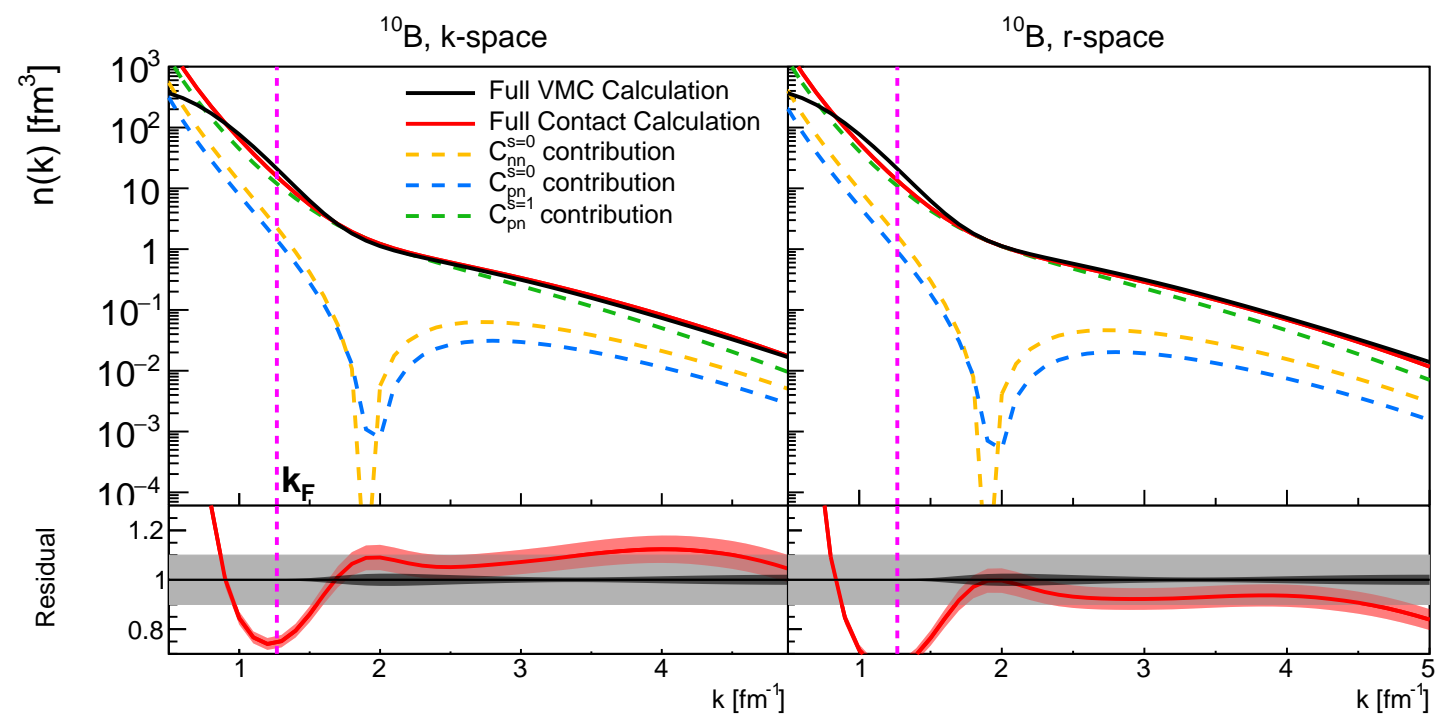

Figure D-4: Same as Fig. D-1, in the case of ${ }^{10} \mathrm{~B}$. 


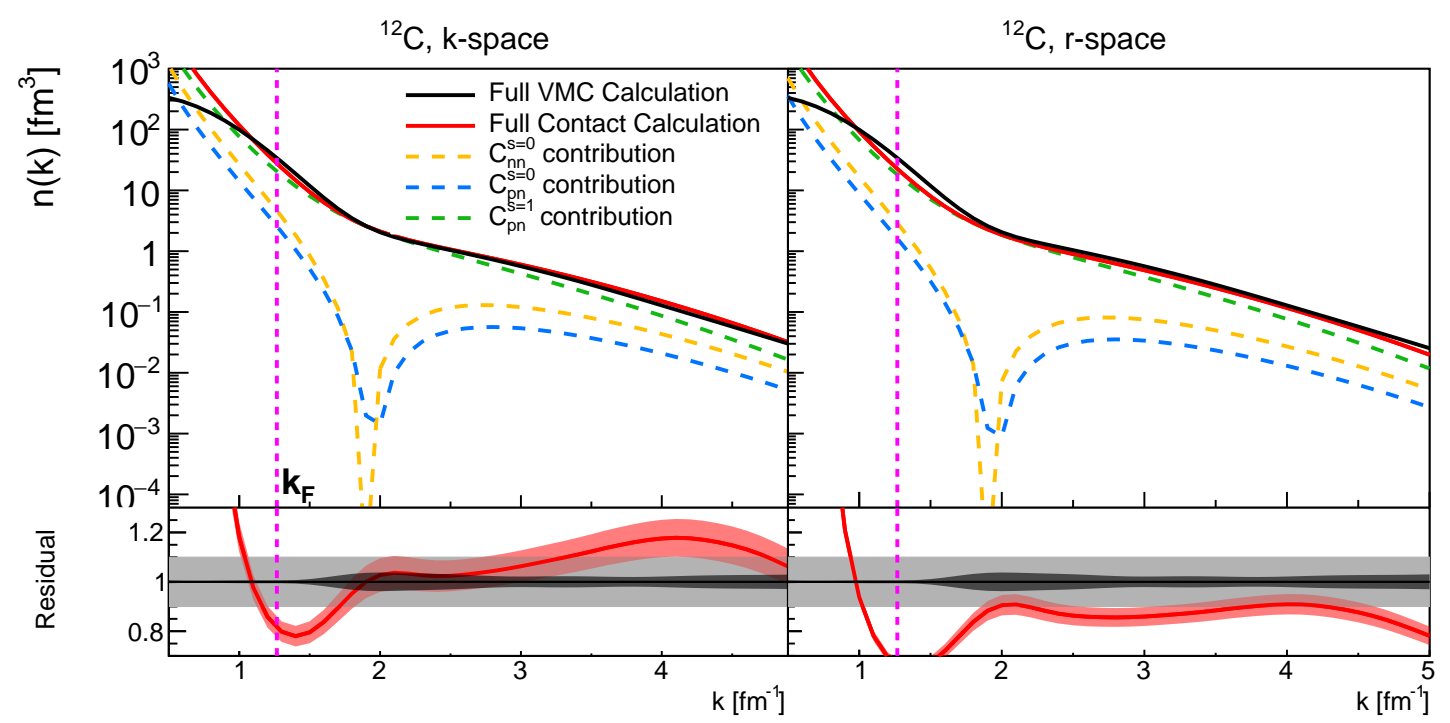

Figure D-5: Same as Fig. D-1, in the case of ${ }^{12} \mathrm{C}$.

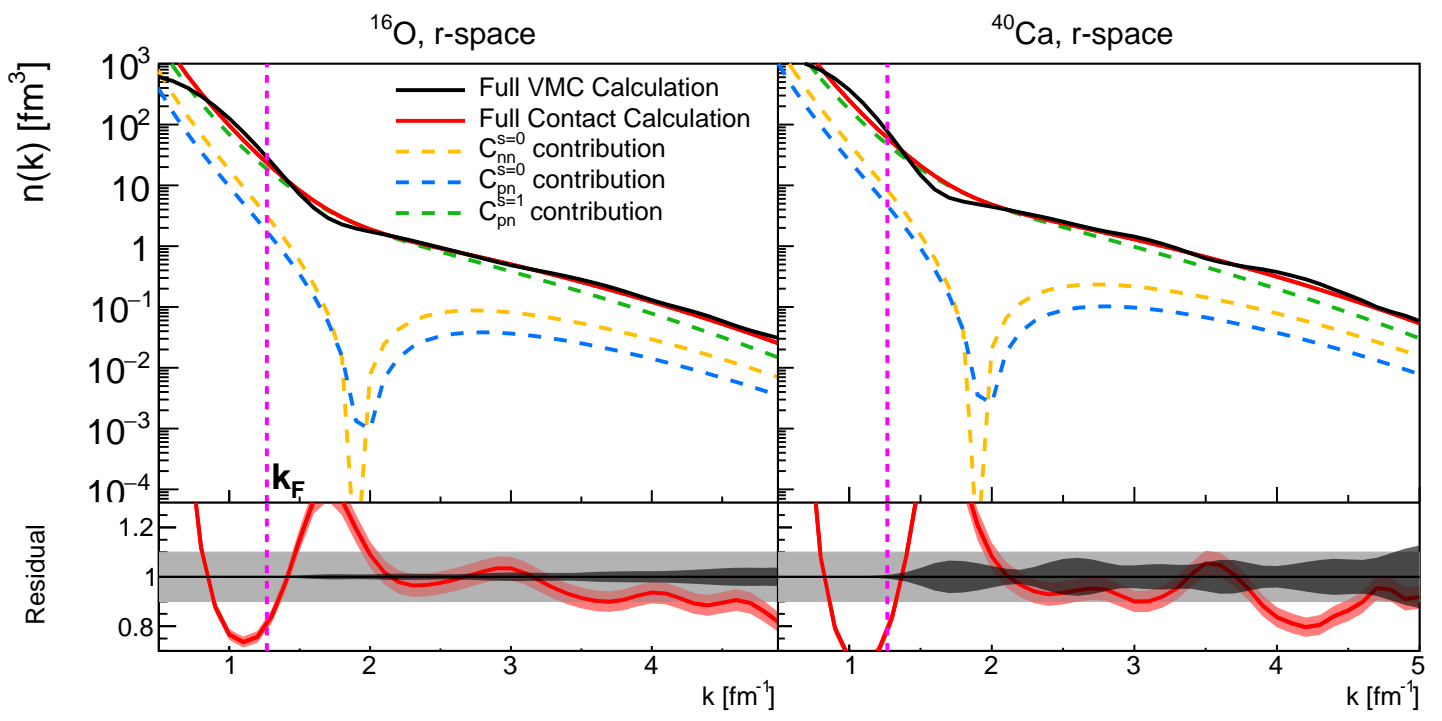

Figure D-6: Comparison between 1-body momentum distributions from VMC and the corresponding GCF distributions determined from contacts extracted in coordinatespace for ${ }^{16} \mathrm{O}$ (left) and ${ }^{40} \mathrm{Ca}$ (right). 


\section{Appendix E}

\section{Generalized Contact Formalism ${ }^{4} \mathrm{He}$ Fit Examples}

${ }^{4} \mathrm{He}$ fits in coordinate- (left) and momentum-space (right) for different $N N+3 N$ potentials. The fit ranges are shown as vertical gray bands. The darker vertical gray bands represent the ranges within which the fit limits are changed to extract the systematic uncertainty for the contacts. This systematic uncertainty is included in the blue bands The VMC distribution error is added as an uncertainty band to the horizontal red line which has a value of 1 . The horizontal dashed lines correspond to a $\pm 20 \%$ deviation.
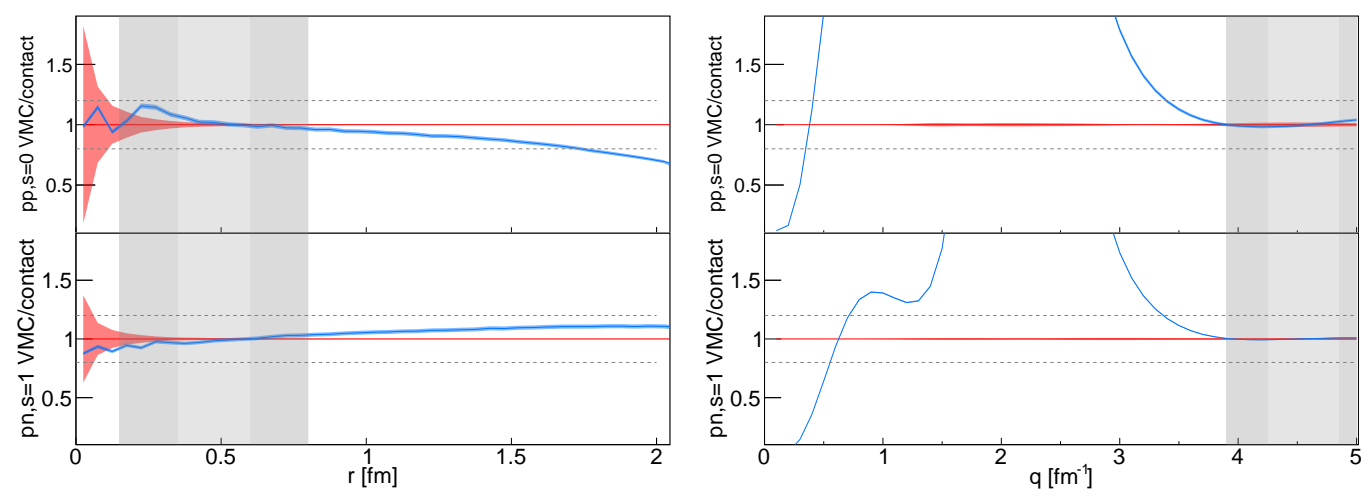

Figure E-1: ${ }^{4} \mathrm{He}$ fits in coordinate- (left) and momentum-space (right) for the $\mathrm{AV} 4$ ' $+\mathrm{UIX}_{\mathrm{c}}$ potential. 

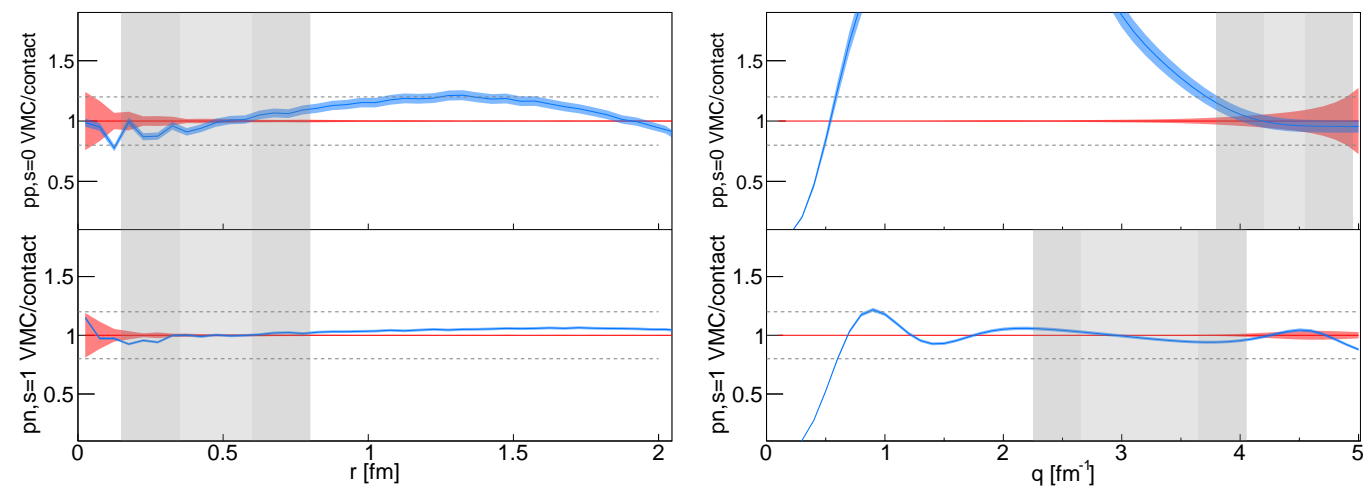

Figure E-2: Same as Fig. E-1 for the $\mathrm{N}^{2} \mathrm{LO}(1.0 \mathrm{fm}, E \mathbb{1})$ potential.
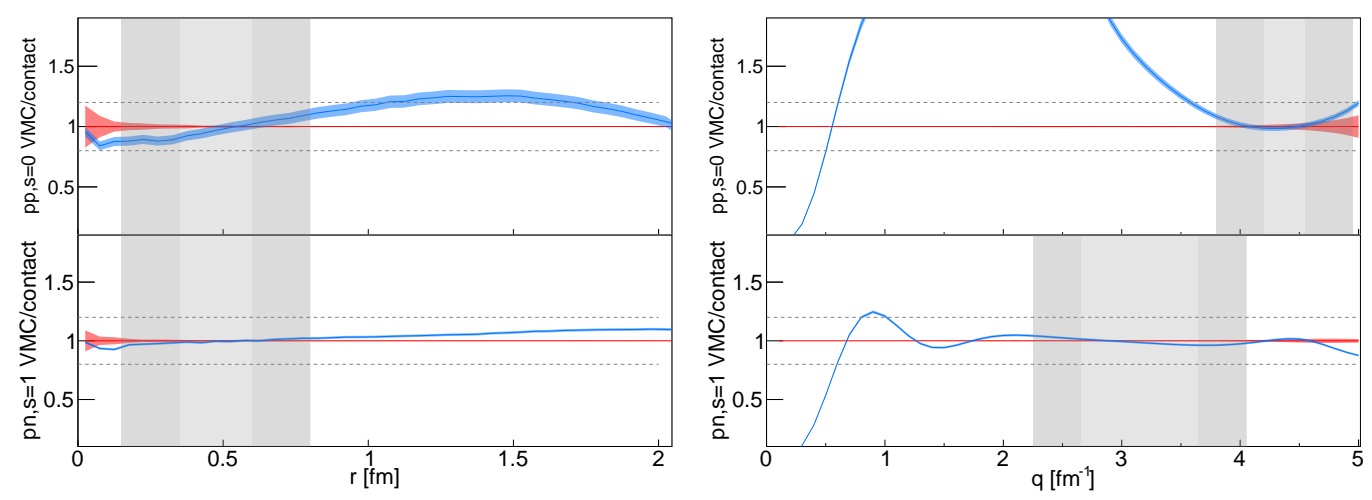

Figure E-3: Same as Fig. E-1 for the $\mathrm{N}^{2} \mathrm{LO}(1.0 \mathrm{fm}, E \tau)$ potential.
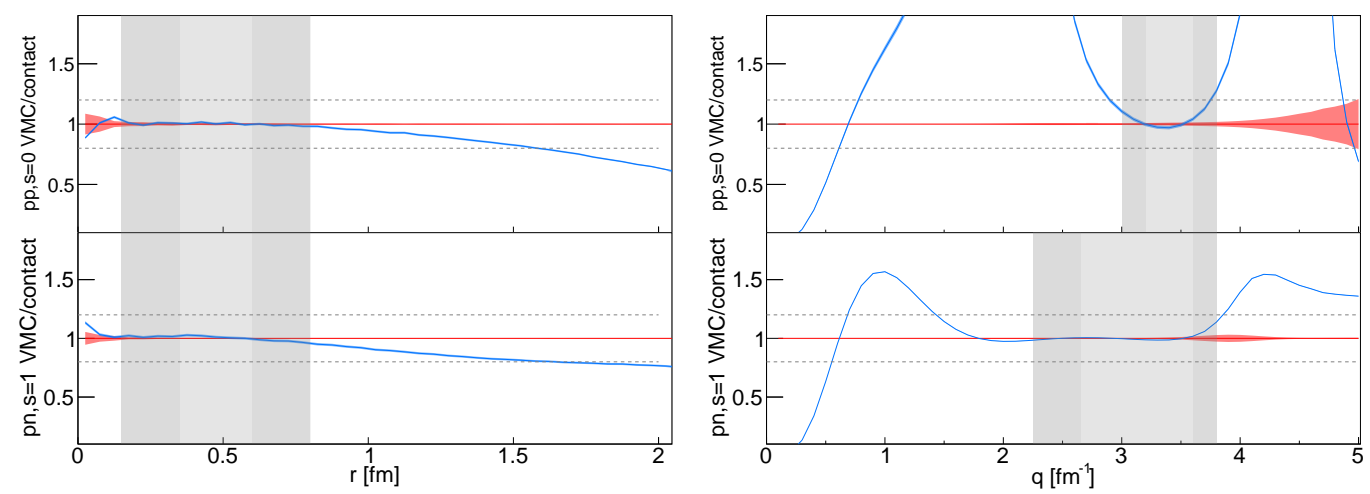

Figure E-4: Same as Fig. E-1 for the $\mathrm{N}^{2} \mathrm{LO}(1.2 \mathrm{fm}, E \mathbb{1})$ potential. 

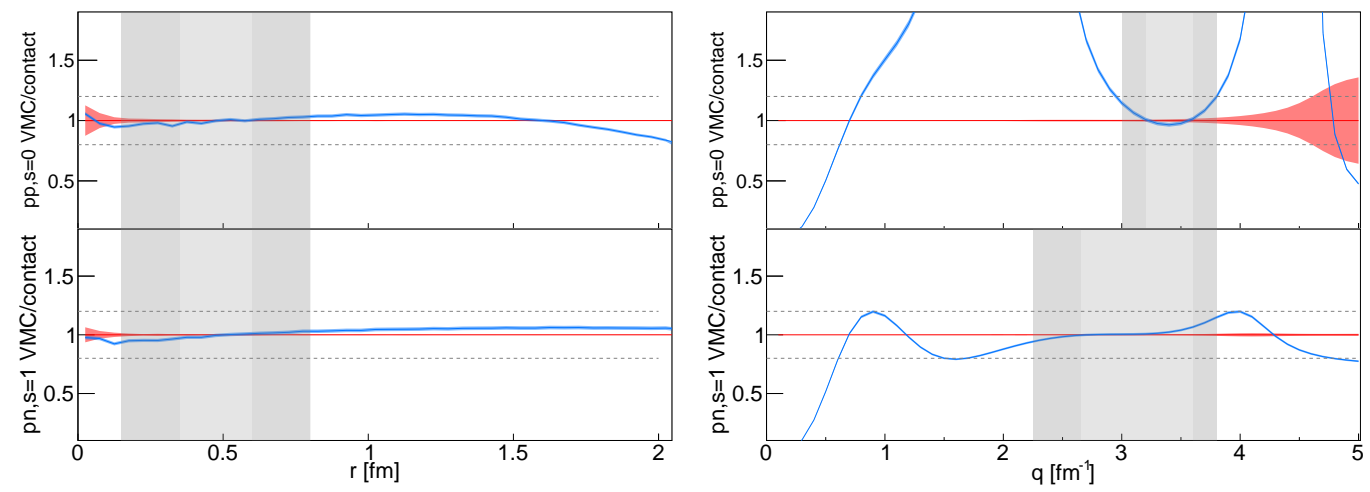

Figure E-5: Same as Fig. E-1 for the $\mathrm{N}^{2} \mathrm{LO}(1.2 \mathrm{fm}, E \tau)$ potential. 


\section{Bibliography}

[1] J. Chadwick, "The Existence of a Neutron," Proc. Roy. Soc. Lond., vol. A136, no. 830, pp. 692-708, 1932.

[2] H. Yukawa, "On the Interaction of Elementary Particles I," Proc. Phys. Math. Soc. Jap., vol. 17, pp. 48-57, 1935. [Prog. Theor. Phys. Suppl.1,1(1935)].

[3] R. Machleidt and I. Slaus, "The Nucleon-nucleon interaction: Topical review," J. Phys., vol. G27, pp. R69-R108, 2001.

[4] R. B. Wiringa, V. G. J. Stoks, and R. Schiavilla, "An Accurate nucleon-nucleon potential with charge independence breaking," Phys. Rev., vol. C51, pp. 38-51, 1995.

[5] R. B. Wiringa and S. C. Pieper, "Evolution of nuclear spectra with nuclear forces," Phys. Rev. Lett., vol. 89, p. 182501, 2002.

[6] A. Gezerlis, I. Tews, E. Epelbaum, S. Gandolfi, K. Hebeler, A. Nogga, and A. Schwenk, "Quantum Monte Carlo Calculations with Chiral Effective Field Theory Interactions," Phys. Rev. Lett., vol. 111, no. 3, p. 032501, 2013.

[7] A. Gezerlis, I. Tews, E. Epelbaum, M. Freunek, S. Gandolfi, K. Hebeler, A. Nogga, and A. Schwenk, "Local chiral effective field theory interactions and quantum Monte Carlo applications," Phys. Rev., vol. C90, no. 5, p. 054323, 2014.

[8] J. Carlson, V. R. Pandharipande, and R. B. Wiringa, "Three-nucleon interaction in 3-body, 4-body, and infinite-body systems," Nucl. Phys., vol. A401, pp. 5985, 1983.

[9] B. S. Pudliner, V. R. Pandharipande, J. Carlson, S. C. Pieper, and R. B. Wiringa, "Quantum Monte Carlo calculations of nuclei with $\mathrm{A}<=7$," Phys. Rev., vol. C56, pp. 1720-1750, 1997.

[10] R. Machleidt and D. R. Entem, "Chiral effective field theory and nuclear forces," Phys. Rept., vol. 503, pp. 1-75, 2011.

[11] D. Lonardoni, S. Gandolfi, J. E. Lynn, C. Petrie, J. Carlson, K. E. Schmidt, and A. Schwenk, "Auxiliary field diffusion Monte Carlo calculations of light and 
medium-mass nuclei with local chiral interactions," Phys. Rev., vol. C97, no. 4, p. 044318, 2018.

[12] M. Piarulli et al., "Light-nuclei spectra from chiral dynamics," Phys. Rev. Lett., vol. 120, no. 5, p. 052503, 2018.

[13] J. E. Lynn, I. Tews, S. Gandolfi, and A. Lovato, "Quantum Monte Carlo Methods in Nuclear Physics: Recent Advances," Submitted to: Ann. Rev. Nucl. Part. Sci., 2019.

[14] J. A. Carlson and R. B. Wiringa, Variational Monte Carlo Techniques in Nuclear Physics, pp. 171-189. Springer Berlin Heidelberg, 1991.

[15] I. Kosztin, B. Faber, and K. Schulten, "Introduction to the diffusion Monte Carlo method," Am. J. Phys., vol. 64, p. 633, 1996.

[16] D. Lonardoni, A. Lovato, S. C. Pieper, and R. B. Wiringa, "Variational calculation of the ground state of closed-shell nuclei up to $A=40$," Phys. Rev., vol. C96, no. 2, p. 024326, 2017.

[17] R. Machleidt, "What is wrong with our current nuclear forces?" Nucl. Theor., vol. 37, pp. 62-67, 2018.

[18] E. J. Moniz, I. Sick, R. R. Whitney, J. R. Ficenec, R. D. Kephart, and W. P. Trower, "Nuclear Fermi momenta from quasielastic electron scattering," Phys. Rev. Lett., vol. 26, pp. 445-448, 1971.

[19] O. Hen, G. A. Miller, E. Piasetzky, and L. B. Weinstein, "Nucleon-Nucleon Correlations, Short-lived Excitations, and the Quarks Within," Rev. Mod. Phys., vol. 89, no. 4, p. 045002, 2017.

[20] G. A. Miller, A. Beck, S. May-Tal Beck, L. B. Weinstein, E. Piasetzky, and O. Hen, "Can Long-Range Nuclear Properties Be Influenced By Short Range Interactions? A chiral dynamics estimate," Phys. Lett., vol. B793, pp. 360-364, 2019.

[21] M. Kortelainen and J. Suhonen, "Improved short-range correlations and $0 \nu \beta \beta$ nuclear matrix elements of ${ }^{76} \mathrm{Ge}$ and ${ }^{82} \mathrm{Se}, "$ Phys. Rev. C, vol. 75, p. 051303, May 2007.

[22] M. Kortelainen and J. Suhonen, "Nuclear matrix elements of $0 \nu \beta \beta$ decay with improved short-range correlations," Phys. Rev. C, vol. 76, p. 024315, Aug 2007.

[23] J. Menendez, A. Poves, E. Caurier, and F. Nowacki, "Disassembling the Nuclear Matrix Elements of the Neutrinoless beta beta Decay," Nucl. Phys., vol. A818, pp. 139-151, 2009.

[24] F. Šimkovic, A. Faessler, H. Müther, V. Rodin, and M. Stauf, " $0 \nu \beta \beta$-decay nuclear matrix elements with self-consistent short-range correlations," Phys. Rev. C, vol. 79, p. 055501, May 2009. 
[25] O. Benhar, R. Biondi, and E. Speranza, "Short-range correlation effects on the nuclear matrix element of neutrinoless double- $\beta$ decay," Phys. Rev., vol. C90, no. 6, p. 065504, 2014.

[26] X. B. Wang, A. C. Hayes, J. Carlson, G. X. Dong, E. Mereghetti, S. Pastore, and R. B. Wiringa, "Comparison between Variational Monte Carlo and Shell Model Calculations of Neutrinoless Double Beta Decay Matrix Elements in Light Nuclei," 2019.

[27] L. B. Weinstein, E. Piasetzky, D. W. Higinbotham, J. Gomez, O. Hen, and R. Shneor, "Short Range Correlations and the EMC Effect," Phys. Rev. Lett., vol. 106, p. 052301, 2011.

[28] O. Hen, E. Piasetzky, and L. B. Weinstein, "New data strengthen the connection between Short Range Correlations and the EMC effect," Phys. Rev., vol. C85, p. 047301, 2012.

[29] O. Hen, D. W. Higinbotham, G. A. Miller, E. Piasetzky, and L. B. Weinstein, "The EMC Effect and High Momentum Nucleons in Nuclei," Int. J. Mod. Phys., vol. E22, p. 1330017, 2013.

[30] B. Schmookler et al., "Modified structure of protons and neutrons in correlated pairs," Nature, vol. 566, no. 7744, pp. 354-358, 2019.

[31] B.-A. Li, B.-J. Cai, L.-W. Chen, and J. Xu, "Nucleon Effective Masses in Neutron-Rich Matter," Prog. Part. Nucl. Phys., vol. 99, pp. 29-119, 2018.

[32] O. Hen, B.-A. Li, W.-J. Guo, L. B. Weinstein, and E. Piasetzky, "Symmetry Energy of Nucleonic Matter With Tensor Correlations," Phys. Rev., vol. C91, no. 2, p. 025803, 2015.

[33] L. Frankfurt, M. Sargsian, and M. Strikman, "Recent observation of short range nucleon correlations in nuclei and their implications for the structure of nuclei and neutron stars," Int. J. Mod. Phys., vol. A23, pp. 2991-3055, 2008.

[34] R. Jastrow, "Many-Body Problem with Strong Forces," Phys. Rev., vol. 98, pp. 1479-1484, 1955.

[35] B. Frois and C. N. Papanicolas, "Electron Scattering and Nuclear Structure," Ann. Rev. Nucl. Part. Sci., vol. 37, pp. 133-176, 1987.

[36] L. Lapikas, "Quasi-elastic electron scattering off nuclei," Nucl. Phys., vol. A553, pp. 297c-308c, 1993.

[37] G. A. Rijsdijk, K. Allaart, and W. H. Dickhoff, "Hole spectral functions and collective excitations," Nucl. Phys., vol. A550, pp. 159-178, 1992.

[38] L. L. Frankfurt, M. I. Strikman, D. B. Day, and M. Sargsian, "Evidence for short range correlations from high $\mathrm{Q}^{* *} 2$ (e, e-prime) reactions," Phys. Rev., vol. C48, pp. 2451-2461, 1993. 
[39] K. S. Egiyan et al., "Observation of nuclear scaling in the A(e, e-prime) reaction at x(B) greater than 1," Phys. Rev., vol. C68, p. 014313, 2003.

[40] K. S. Egiyan et al., "Measurement of 2- and 3-nucleon short range correlation probabilities in nuclei," Phys. Rev. Lett., vol. 96, p. 082501, 2006.

[41] N. Fomin et al., "New measurements of high-momentum nucleons and shortrange structures in nuclei," Phys. Rev. Lett., vol. 108, p. 092502, 2012.

[42] F. Benmokhtar et al., "Measurement of the He-3(e,e-prime p)pn reaction at high missing energies and momenta," Phys. Rev. Lett., vol. 94, p. 082305, 2005.

[43] J. M. Laget, "The Electro-disintegration of few body systems revisited," Phys. Lett., vol. B609, pp. 49-56, 2005.

[44] M. M. Rvachev et al., "The Quasielastic He-3(e,e'p)d reaction at $\mathrm{Q}^{* * 2}=1.5$ $\mathrm{GeV}^{* * 2}$ for recoil momenta up to $1-\mathrm{GeV} / \mathrm{c}$," Phys. Rev. Lett., vol. 94, p. 192302, 2005 .

[45] W. U. Boeglin et al., "Probing the high momentum component of the deuteron at high $Q^{2}, "$ Phys. Rev. Lett., vol. 107, p. 262501, 2011.

[46] D. Rohe et al., "Correlated strength in nuclear spectral function," Phys. Rev. Lett., vol. 93, p. 182501, 2004.

[47] A. Tang et al., "n-p short range correlations from (p,2p + n) measurements," Phys. Rev. Lett., vol. 90, p. 042301, 2003.

[48] J. Aclander et al., "The large momentum transfer reaction $\mathrm{C}-12(\mathrm{p}, 2 \mathrm{p}+\mathrm{n})$ as a new method for measuring short range N N correlations in nuclei," Phys. Lett., vol. B453, pp. 211-216, 1999.

[49] E. Piasetzky, M. Sargsian, L. Frankfurt, M. Strikman, and J. W. Watson, "Evidence for the strong dominance of proton-neutron correlations in nuclei," Phys. Rev. Lett., vol. 97, p. 162504, 2006.

[50] E. O. Cohen et al., "Center of Mass Motion of Short-Range Correlated Nucleon Pairs studied via the A(e,epp) Reaction," Phys. Rev. Lett., vol. 121, no. 9, p. 092501, 2018.

[51] R. Shneor et al., "Investigation of proton-proton short-range correlations via the C-12(e, e-prime pp) reaction," Phys. Rev. Lett., vol. 99, p. 072501, 2007.

[52] C. Ciofi degli Atti and S. Simula, "Realistic model of the nucleon spectral function in few and many nucleon systems," Phys. Rev., vol. C53, p. 1689, 1996.

[53] C. Colle, W. Cosyn, J. Ryckebusch, and M. Vanhalst, "Factorization of exclusive electron-induced two-nucleon knockout," Phys. Rev., vol. C89, no. 2, p. 024603 , 2014. 
[54] M. Vanhalst, W. Cosyn, and J. Ryckebusch, "Counting the amount of correlated pairs in a nucleus," Phys. Rev., vol. C84, p. 031302, 2011.

[55] M. Vanhalst, J. Ryckebusch, and W. Cosyn, "Quantifying short-range correlations in nuclei," Phys. Rev., vol. C86, p. 044619, 2012.

[56] I. Korover et al., "Probing the Repulsive Core of the Nucleon-Nucleon Interaction via the ${ }^{4} \mathrm{He}\left(e, e^{\prime} p N\right)$ Triple-Coincidence Reaction," Phys. Rev. Lett., vol. 113, no. 2, p. 022501, 2014.

[57] R. Subedi et al., "Probing Cold Dense Nuclear Matter," Science, vol. 320, pp. 1476-1478, 2008.

[58] M. Duer et al., "Direct Observation of Proton-Neutron Short-Range Correlation Dominance in Heavy Nuclei," Phys. Rev. Lett., vol. 122, no. 17, p. 172502, 2019.

[59] O. Hen et al., "Momentum sharing in imbalanced Fermi systems," Science, vol. 346, pp. 614-617, 2014.

[60] M. Duer et al., "Probing high-momentum protons and neutrons in neutron-rich nuclei," Nature, vol. 560, no. 7720, pp. 617-621, 2018.

[61] R. Schiavilla, R. B. Wiringa, S. C. Pieper, and J. Carlson, "Tensor Forces and the Ground-State Structure of Nuclei," Phys. Rev. Lett., vol. 98, p. 132501, 2007.

[62] M. Alvioli, C. Ciofi degli Atti, and H. Morita, "Proton-neutron and protonproton correlations in medium-weight nuclei and the role of the tensor force," Phys. Rev. Lett., vol. 100, p. 162503, 2008.

[63] A. Schmidt et al., "Probing the core of the strong nuclear interaction," Nature, vol. 578, no. 7796, pp. 540-544, 2020.

[64] I. Korover et al., "Tensor-to-scalar transition in the nucleon-nucleon interaction mapped by ${ }^{12} \mathrm{C}\left(e, e^{\prime} p n\right)$ measurements." In preparation.

[65] H. Baghdasaryan et al., "Tensor Correlations Measured in ${ }^{3} \mathrm{He}\left(e, e^{\prime} p p\right) n$," Phys. Rev. Lett., vol. 105, p. 222501, 2010.

[66] R. B. Wiringa, R. Schiavilla, S. C. Pieper, and J. Carlson, "Nucleon and nucleonpair momentum distributions in $A \leq 12$ nuclei," Phys. Rev., vol. C89, no. 2, p. 024305, 2014.

[67] J. Golak, H. Kamada, H. Witala, W. Gloeckle, and S. Ishikawa, "Electron induced $\mathrm{p} \mathrm{d}$ and $\mathrm{p} \mathrm{p} \mathrm{n}$ breakup of He-3 with full inclusion of final-state interactions," Phys. Rev., vol. C51, pp. 1638-1647, 1995.

[68] O. Benhar, D. Day, and I. Sick, "Inclusive quasi-elastic electron-nucleus scattering," Rev. Mod. Phys., vol. 80, pp. 189-224, 2008. 
[69] C. Ciofi degli Atti and L. P. Kaptari, "Calculations of the exclusive processes H-2(e, e-prime p)n, He-3(e, e-prime p)H-2 and He-3(e, e-prime p)(pn) within a generalized Glauber approach," Phys. Rev., vol. C71, p. 024005, 2005.

[70] J. J. Kelly, "Nucleon knockout by intermediate-energy electrons," Adv. Nucl. Phys., vol. 23, pp. 75-294, 1996. [,75(1996)].

[71] T. De Forest, "Off-Shell electron Nucleon Cross-Sections. The Impulse Approximation," Nucl. Phys., vol. A392, pp. 232-248, 1983.

[72] J. Golak, R. Skibinski, H. Witala, W. Glockle, A. Nogga, and H. Kamada, "Electron and photon scattering on three-nucleon bound states," Phys. Rept., vol. 415, pp. 89-205, 2005.

[73] D. Lonardoni, S. Gandolfi, X. B. Wang, and J. Carlson, "Single- and twonucleon momentum distributions for local chiral interactions," Phys. Rev. C, vol. 98, p. 014322, Jul 2018.

[74] M. M. Sargsian, "Selected topics in high energy semiexclusive electronuclear reactions," Int. J. Mod. Phys., vol. E10, pp. 405-458, 2001.

[75] M. M. Sargsian et al., "Hadrons in the nuclear medium," J. Phys., vol. G29, no. 3, pp. R1-R45, 2003.

[76] C. Carasco et al., "Final state interaction effects in polarized-He-3(polarized-e, e-prime p)," Phys. Lett., vol. B559, pp. 41-48, 2003.

[77] J. Bermuth et al., "The Neutron charge form-factor and target analyzing powers from polarized-He-3 (polarized-e,e-prime n) scattering," Phys. Lett., vol. B564, pp. 199-204, 2003.

[78] M. M. Sargsian, "Private communication."

[79] M. M. Sargsian, T. V. Abrahamyan, M. I. Strikman, and L. L. Frankfurt, "Exclusive electrodisintegration of ${ }^{3} \mathrm{He}$ at high $Q^{2}$. i. generalized eikonal approximation," Phys. Rev. C, vol. 71, p. 044614, Apr 2005.

[80] M. M. Sargsian, T. V. Abrahamyan, M. I. Strikman, and L. L. Frankfurt, "Exclusive electrodisintegration of ${ }^{3} \mathrm{He}$ at high $Q^{2}$. ii. decay function formalism," Phys. Rev. C, vol. 71, p. 044615, Apr 2005.

[81] I. Sick, "Elastic electron scattering from light nuclei," Prog. Part. Nucl. Phys., vol. 47, pp. 245-318, 2001.

[82] E. Long et al., "Measurement of the single-spin asymmetry $A_{y}^{0}$ in quasi-elastic ${ }^{3} \mathrm{He}^{\uparrow}\left(e, e^{\prime} n\right)$ scattering at $0.4<Q^{2}<1.0 \mathrm{GeV} / c^{2}$," Phys. Lett., vol. B797, p. $134875,2019$. 
[83] M. Mihovilovic et al., "Measurement of double-polarization asymmetries in the quasielastic ${ }^{3} \mathrm{He}\left(\overrightarrow{\mathrm{e}}, \mathrm{e}^{\prime} \mathrm{d}\right)$ process," Phys. Rev. Lett., vol. 113, no. 23, p. 232505, 2014.

[84] M. Mihovilovič et al., "Measurement of double-polarization asymmetries in the quasi-elastic ${ }^{3} \mathrm{He}\left(\overrightarrow{\mathrm{e}}, \mathrm{e}^{\prime} \mathrm{p}\right)$ process," Phys. Lett., vol. B788, pp. 117-121, 2019.

[85] Y. W. Zhang et al., "Measurement of the Target-Normal Single-Spin Asymmetry in Quasielastic Scattering from the Reaction ${ }^{3} \mathrm{He}^{\uparrow}\left(e, e^{\prime}\right)$," Phys. Rev. Lett., vol. 115, no. 17, p. 172502, 2015.

[86] A. Camsonne et al., "JLab Measurements of the ${ }^{3}$ He Form Factors at Large Momentum Transfers," Phys. Rev. Lett., vol. 119, no. 16, p. 162501, 2017. [Addendum: Phys. Rev. Lett.119,no.20,209901(2017)].

[87] S. Riordan et al., "Measurements of the Electric Form Factor of the Neutron up to $Q^{2}=3.4 \mathrm{GeV}^{2}$ using the Reaction ${ }^{3} \mathrm{He}^{->}\left(e^{->}, e^{\prime} n\right) p p$," Phys. Rev. Lett., vol. 105, p. 262302, 2010.

[88] C. Ciofi degli Atti and L. P. Kaptari, "On the interpretation of the processes He-3(e,e-prime p) H-2 and He-3(e,e-prime p)(pn) at high missing momenta," Phys. Rev. Lett., vol. 95, p. 052502, 2005.

[89] M. Alvioli, C. Ciofi degli Atti, and L. P. Kaptari, "Calculation of the cross section and the transverse-longitudinal asymmetry of the process 3-He (e, eprime, p) pn at medium energies within the unfactorized generalized Glauber approach," Phys. Rev., vol. C81, p. 021001, 2010.

[90] H. Collard, R. Hofstadter, A. Johansson, R. Parks, M. Ryneveld, A. Walker, M. R. Yearian, R. B. Day, and R. T. Wagner, "Electron Scattering in Tritium and He-3," Phys. Rev. Lett., vol. 11, pp. 132-134, 1963.

[91] L. I. Schiff, H. Collard, R. Hofstadter, A. Johansson, and M. R. Yearian, "Analysis of H-3 and He-3 Form Factors and the Determination of the Charge Form Factor of the Neutron," Phys. Rev. Lett., vol. 11, pp. 387-390, 1963.

[92] A. Johansson, "Quasifree electron-proton scattering in $\mathrm{h}^{3}$ and $\mathrm{he}^{3}$," Phys. Rev., vol. 136, pp. B1030-B1035, Nov 1964.

[93] T. A. GRIFFY and R. J. OAKES, "Structure of he $\mathrm{h}^{3}$ and $\mathrm{h}^{3}$ from high-energy electron scattering," Rev. Mod. Phys., vol. 37, pp. 402-405, Jul 1965.

[94] K. Dow, "The Bates trinucleon experiment - inelastic scattering," Lect. Notes Phys., vol. 260, pp. 346-348, 1986.

[95] D. H. Beck, "The Bates trinucleon experiment - elastic scattering," Lect. Notes Phys., vol. 260, pp. 138-143, 1986. 
[96] D. Beck et al., "Isoscalar and isovector form factors of H-3 and He-3 for Q below 2.9 fm-1 from electron-scattering measurements," Phys. Rev. Lett., vol. 59, pp. 1537-1540, 1987.

[97] K. Dow et al., "Longitudinal Response Functions and Sum Rules for Quasielastic Electron Scattering From ${ }^{3} \mathrm{H}$ and ${ }^{3} \mathrm{He}$," Phys. Rev. Lett., vol. 61, pp. 1706-1709, 1988.

[98] F. P. Juster et al., "TRITIUM ELECTROMAGNETIC FORM-FACTORS," Phys. Rev. Lett., vol. 55, pp. 2261-2264, 1985.

[99] A. Amroun et al., "H-3 and He-3 electromagnetic form-factors," Nucl. Phys., vol. A579, pp. 596-626, 1994.

[100] A. Kievsky, E. Pace, G. Salme, and M. Viviani, "Neutron electromagnetic formfactors and inclusive scattering of polarized electrons by polarized He-3 and He-3 targets," Phys. Rev., vol. C56, pp. 64-75, 1997.

[101] R. Cruz-Torres et al., "Comparing proton momentum distributions in $A=2$ and 3 nuclei via ${ }^{2} \mathrm{H}^{3} \mathrm{H}$ and ${ }^{3} \mathrm{He}\left(e, e^{\prime} p\right)$ measurements," Phys. Lett., vol. B797, p. $134890,2019$.

[102] R. Cruz-Torres et al., "Probing few-body nuclear dynamics via $3 \mathrm{H}$ and $3 \mathrm{He}$ (e,e'p)pn cross-section measurements," 2020.

[103] C. W. Leemann, D. R. Douglas, and G. A. Krafft, "The Continuous Electron Beam Accelerator Facility: CEBAF at the Jefferson Laboratory," Ann. Rev. Nucl. Part. Sci., vol. 51, pp. 413-450, 2001.

[104] J. Alcorn et al., "Basic Instrumentation for Hall A at Jefferson Lab," Nucl. Instrum. Meth., vol. A522, pp. 294-346, 2004.

[105] J. Berthot and P. Vernin, "Beam energy measurement in Hall-A of CEBAF," Nucl. Phys. News, vol. 9N4, pp. 12-16, 1999.

[106] J. Denard, A. Saha, and G. Lavessiere, "High Accuracy Beam Current Monitor System for CEBAF's Experimental Hall A," Conf. Proc., vol. C0106181, pp. 2326-2328, 2001. [,2326(2001)].

[107] D. Meekins, "Hall a tritium target configuration for december 2017." TGTRPT-17-007.

[108] D. Meekins, "Hall a tritium target: Engineering report." TGT-RPT-17-003.

[109] S. N. Santiesteban et al., "Density Changes in Low Pressure Gas Targets for Electron Scattering Experiments," Nucl. Instrum. Meth., vol. A940, pp. 351$358,2019$.

[110] D. Meekins, "Determination of solid and fluid target thickness from measurements." TGT-CALC-17-020. 
[111] K. G. Fissum et al., "Vertical drift chambers for the Hall A high-resolution spectrometers at Jefferson Lab," Nucl. Instrum. Meth., vol. A474, pp. 108-131, 2001.

[112] W. R. Leo, Techniques for Nuclear and Particle Physics Experiments: A How to Approach. 1987.

[113] M. Iodice et al., "The CO-2 gas Cherenkov detectors for the Jefferson Lab Hall-A spectrometers," Nucl. Instrum. Meth., vol. A411, pp. 223-237, 1998.

[114] "SIMC." https://hallcweb.jlab.org/wiki/index.php/SIMC_Monte_Carlo. Accessed: 2020-02-05.

[115] R. B. Wiringa, "Variational calculations of few-body nuclei," Phys. Rev., vol. C43, pp. 1585-1598, 1991.

[116] O. Benhar, A. Fabrocini, S. Fantoni, and I. Sick, "Spectral function of finite nuclei and scattering of GeV electrons," Nucl. Phys., vol. A579, pp. 493-517, 1994.

[117] N. Liyanage, "Optics calibration of the hall a high resolution spectrometers using the new optimizer." JLAB-TN-02-012.

[118] L. Ou, Precision Measurements of Electron-Proton Elastic Scattering Cross Sections at Large $Q^{2}$. PhD thesis, MIT, 2019.

[119] L. W. Mo and Y.-S. Tsai, "Radiative Corrections to Elastic and Inelastic e p and mu p Scattering," Rev. Mod. Phys., vol. 41, pp. 205-235, 1969.

[120] E. L. Lomon, "Effect of revised R(n) measurements on extended GariKrumpelmann model fits to nucleon electromagnetic form factors," 2006.

[121] L. L. Lucas and M. P. Unterweger, "Comprehensive review and critical evaluation of the half-life of tritium," Journal of Research of the National Institute of Standards and Technology, vol. 105, no. 4, pp. 541-549, 2000.

[122] J. S. Schwinger, "Quantum electrodynamics. III: The electromagnetic properties of the electron: Radiative corrections to scattering," Phys. Rev., vol. 76, pp. 790-817, 1949. [,88(1949)].

[123] N. C. R. Makins, Measurement of the Nuclear Dependence and Momentum Transfer Dependence of Quasielastic ( $\left.e, e^{\prime} p\right)$ Scattering at Large Momentum Transfer. PhD thesis, MIT, 1994.

[124] R. Ent, B. W. Filippone, N. C. R. Makins, R. G. Milner, T. G. O'Neill, and D. A. Wasson, "Radiative corrections for (e, e-prime p) reactions at $\mathrm{GeV}$ energies," Phys. Rev., vol. C64, p. 054610, 2001.

[125] O. Benhar and V. R. Pandharipande, "Scattering of GeV electrons by light nuclei," Phys. Rev., vol. C47, pp. 2218-2227, 1993. 
[126] L. E. Marcucci, F. Sammarruca, M. Viviani, and R. Machleidt, "Momentum distributions and short-range correlations in the deuteron and ${ }^{3} \mathrm{He}$ with modern chiral potentials," Phys. Rev., vol. C99, no. 3, p. 034003, 2019.

[127] A. Kievsky, S. Rosati, M. Viviani, L. E. Marcucci, and L. Girlanda, "A Highprecision variational approach to three- and four-nucleon bound and zero-energy scattering states," J. Phys., vol. G35, p. 063101, 2008.

[128] S. N. More, S. K. Bogner, and R. J. Furnstahl, "Scale dependence of deuteron electrodisintegration," Phys. Rev. C, vol. 96, p. 054004, Nov 2017.

[129] J. Gao et al., "Dynamical relativistic effects in quasielastic $1 p$-shell proton knockout from ${ }^{16}$ O," Phys. Rev. Lett., vol. 84, pp. 3265-3269, Apr 2000.

[130] J. M. Udias, J. A. Caballero, E. Moya de Guerra, J. E. Amaro, and T. W. Donnelly, "Quasielastic scattering from relativistic bound nucleons: Transverselongitudinal response," Phys. Rev. Lett., vol. 83, pp. 5451-5454, Dec 1999.

[131] R. Alvarez-Rodriguez, J. M. Udias, J. R. Vignote, E. Garrido, P. Sarriguren, E. Moya de Guerra, E. Pace, A. Kievsky, and G. Salme, "Relativistic description of 3He(e,e'p)2H," Few Body Syst., vol. 50, pp. 359-362, 2011.

[132] K. G. Fissum et al., "The Dynamics of the quasielastic O-16(e, e-prime p) reaction at $\mathrm{Q}^{* *} 2=0.8(\mathrm{GeV} / \mathrm{c})^{* *} 2$," Phys. Rev., vol. C70, p. 034606, 2004.

[133] J. T. Stewart, J. P. Gaebler, T. E. Drake, and D. S. Jin, "Verification of universal relations in a strongly interacting fermi gas," Phys. Rev. Lett., vol. 104, p. 235301, Jun 2010.

[134] Y. Sagi, T. E. Drake, R. Paudel, and D. S. Jin, "Measurement of the homogeneous contact of a unitary fermi gas," Phys. Rev. Lett., vol. 109, p. 220402, Nov 2012.

[135] G. B. Partridge, K. E. Strecker, R. I. Kamar, M. W. Jack, and R. G. Hulet, "Molecular probe of pairing in the bec-bcs crossover," Phys. Rev. Lett., vol. 95, p. 020404, Jul 2005.

[136] E. D. Kuhnle, H. Hu, X.-J. Liu, P. Dyke, M. Mark, P. D. Drummond, P. Hannaford, and C. J. Vale, "Universal behavior of pair correlations in a strongly interacting fermi gas," Phys. Rev. Lett., vol. 105, p. 070402, Aug 2010.

[137] R. Weiss, B. Bazak, and N. Barnea, "Generalized nuclear contacts and momentum distributions," Phys. Rev., vol. C92, no. 5, p. 054311, 2015.

[138] R. Weiss, R. Cruz-Torres, N. Barnea, E. Piasetzky, and O. Hen, "The nuclear contacts and short range correlations in nuclei," Phys. Lett., vol. B780, pp. 211$215,2018$. 
[139] R. Cruz-Torres, D. Lonardoni, R. Weiss, N. Barnea, D. W. Higinbotham, E. Piasetzky, A. Schmidt, L. B. Weinstein, R. B. Wiringa, and O. Hen, "Scale and Scheme Independence and Position-Momentum Equivalence of Nuclear ShortRange Correlations," 2019.

[140] R. Cruz-Torres, A. Schmidt, G. A. Miller, L. B. Weinstein, N. Barnea, R. Weiss, E. Piasetzky, and O. Hen, "Short range correlations and the isospin dependence of nuclear correlation functions," Phys. Lett., vol. B785, pp. 304-308, 2018.

[141] D. Lonardoni, J. Carlson, S. Gandolfi, J. E. Lynn, K. E. Schmidt, A. Schwenk, and X. Wang, "Properties of nuclei up to $A=16$ using local chiral interactions," Phys. Rev. Lett., vol. 120, no. 12, p. 122502, 2018.

[142] J. E. Lynn, D. Lonardoni, J. Carlson, J. W. Chen, W. Detmold, S. Gandolfi, and A. Schwenk, "Ab initio short-range-correlation scaling factors from light to medium-mass nuclei," J. Phys., vol. G47, no. 4, p. 045109, 2020.

[143] S. Tan, "Large momentum part of a strongly correlated fermi gas," Annals of Physics, vol. 323, no. 12, p. 2971, 2008.

[144] H. A. Bethe and R. Peierls, "Photoelectric Disintegration of the Diplon," in International Conference on Physics, London 1934, a Joint Conference Organized by the International Union of Pure and Applied Physics and the Physical Society. Papers 85 Discussions in Two Volumes. Vol. I: Nuclear Physics. Vol. II: The Solid State of Matter., pp. 93-94, 1935.

[145] F. Werner and Y. Castin, "General relations for quantum gases in two and three dimensions. Two-component fermions," 2012.

[146] O. Hen, L. B. Weinstein, E. Piasetzky, G. A. Miller, M. M. Sargsian, and Y. Sagi, "Correlated fermions in nuclei and ultracold atomic gases," Phys. Rev., vol. C92, no. 4, p. 045205, 2015.

[147] R. Weiss, B. Bazak, and N. Barnea, "Nuclear Neutron-Proton Contact and the Photoabsorption Cross Section," Phys. Rev. Lett., vol. 114, no. 1, p. 012501, 2015.

[148] J. Carlson, S. Gandolfi, F. Pederiva, S. C. Pieper, R. Schiavilla, K. E. Schmidt, and R. B. Wiringa, "Quantum Monte Carlo methods for nuclear physics," Rev. Mod. Phys., vol. 87, p. 1067, 2015.

[149] C. Ciofi degli Atti and H. Morita, "Universality of many-body two-nucleon momentum distributions: Correlated nucleon spectral function of complex nuclei," Phys. Rev., vol. C96, no. 6, p. 064317, 2017.

[150] H. Feldmeier, W. Horiuchi, T. Neff, and Y. Suzuki, "Universality of short-range nucleon-nucleon correlations," Phys. Rev., vol. C84, p. 054003, 2011. 
[151] M. Alvioli, C. Ciofi degli Atti, L. P. Kaptari, C. B. Mezzetti, and H. Morita, "Nucleon momentum distributions, their spin-isospin dependence and shortrange correlations," Phys. Rev., vol. C87, no. 3, p. 034603, 2013.

[152] J.-W. Chen, W. Detmold, J. E. Lynn, and A. Schwenk, "Short Range Correlations and the EMC Effect in Effective Field Theory," Phys. Rev. Lett., vol. 119, no. 26, p. 262502, 2017.

[153] R. Weiss et al., "Study of inclusive electron scattering scaling using the generalized contact formalism." In preparation.

[154] J. Arrington and N. Fomin, "Searching for Flavor Dependence in Nuclear Quark Behavior," Phys. Rev. Lett., vol. 123, p. 042501, Jul 2019.

[155] B.-J. Cai and B.-A. Li, "Symmetry energy of cold nucleonic matter within a relativistic mean field model encapsulating effects of high momentum nucleons induced by short-range correlations," Phys. Rev., vol. C93, no. 1, p. 014619, 2016.

[156] R. Weiss, E. Pazy, and N. Barnea, "Short range correlations - The important role of few-body dynamics in many-body systems," Few Body Syst., vol. 58, no. 1, p. 9, 2017.

[157] R. Weiss, A. Schmidt, G. A. Miller, and N. Barnea, "Short-range correlations and the charge density," Phys. Lett., vol. B790, pp. 484-489, 2019.

[158] R. Weiss, I. Korover, E. Piasetzky, O. Hen, and N. Barnea, "Energy and momentum dependence of nuclear short-range correlations - Spectral function, exclusive scattering experiments and the contact formalism," Phys. Lett., vol. B791, pp. 242-248, 2019.

[159] Y. Hatta, M. Strikman, J. Xu, and F. Yuan, "Sub-threshold $J / \psi$ and $\Upsilon$ Production in $\gamma A$ Collisions," 2019.

[160] G. A. Miller and J. E. Spencer, "A Survey of Pion Charge-Exchange Reactions with Nuclei," Annals Phys., vol. 100, p. 562, 1976.

[161] M. Kortelainen, O. Civitarese, J. Suhonen, and J. Toivanen, "Short-range correlations and neutrinoless double beta decay," Phys. Lett. B, vol. 647, no. 2, pp. 128-132, 2007.

[162] J. Engel and G. Hagen, "Corrections to the neutrinoless double- $\beta$-decay operator in the shell model," Phys. Rev. C, vol. 79, p. 064317, Jun 2009.

[163] E. G. Adelberger and W. C. Haxton, "Parity Violation in the Nucleon-Nucleon Interaction," Ann. Rev. Nucl. Part. Sci., vol. 35, pp. 501-558, 1985.

[164] I. S. Towner, "Quenching of spin matrix elements in nuclei," Phys. Rept., vol. 155, pp. 263-377, 1987. 
[165] I. Mardor, Y. Mardor, E. Piasetzky, J. Alster, and M. M. Sargsian, "Effect of multiple scattering on the measurement of nuclear transparency," Phys. Rev., vol. C46, pp. 761-767, 1992.

[166] S. Frankel, W. Frati, and N. Walet, "Extracting nuclear transparency from p-A cross-sections," Nucl. Phys., vol. A580, pp. 595-613, 1994.

[167] B. Kundu, P. Jain, J. P. Ralston, and J. Samuelsson, "Hadronic electromagnetic form-factors and color transparency," in Perspectives in hadronic physics. Proceedings, 2nd International Conference, Trieste, Italy, May 10-14, 1999, pp. 87-96, 1999.

[168] B. Kundu, J. Samuelsson, P. Jain, and J. P. Ralston, "Perturbative color transparency in electroproduction experiments," Phys. Rev., vol. D62, p. 113009, 2000.

[169] T. S. H. Lee and G. A. Miller, "Color transparency and high-energy (p, 2 p) nuclear reactions," Phys. Rev., vol. C45, pp. 1863-1870, 1992.

[170] W. Cosyn, M. C. Martínez, and J. Ryckebusch, "Color transparency and shortrange correlations in exclusive pion photo- and electroproduction from nuclei," Phys. Rev. C, vol. 77, p. 034602, Mar 2008.

[171] G. Baym, B. Blättel, L. L. Frankfurt, H. Heiselberg, and M. Strikman, "Correlations and fluctuations in high-energy nuclear collisions," Phys. Rev. C, vol. 52, pp. 1604-1617, Sep 1995.

[172] H. De Vries, C. W. De Jager, and C. De Vries, "Nuclear charge and magnetization density distribution parameters from elastic electron scattering," Atom. Data Nucl. Data Tabl., vol. 36, pp. 495-536, 1987.

[173] J. W. Negele and D. Vautherin, "Density-Matrix Expansion for an Effective Nuclear Hamiltonian," Phys. Rev., vol. C5, pp. 1472-1493, 1972.

[174] J. W. Negele, "Structure of Finite Nuclei in the Local-Density Approximation," Phys. Rev., vol. C1, pp. 1260-1321, 1970.

[175] M. Alvioli, C. Ciofi degli Atti, and H. Morita, "Ground-state energies, densities and momentum distributions in closed-shell nuclei calculated within a cluster expansion approach and realistic interactions," Phys. Rev., vol. C72, p. 054310, 2005.

[176] R. Roth, H. Hergert, P. Papakonstantinou, T. Neff, and H. Feldmeier, "Matrix elements and few-body calculations within the unitary correlation operator method," Phys. Rev., vol. C72, p. 034002, 2005.

[177] J. Engel, J. Carlson, and R. B. Wiringa, "Jastrow functions in double- $\beta$ decay," Phys. Rev. C, vol. 83, p. 034317, Mar 2011. 